\title{
Management of Prelabour Rupture of Membranes at Term, at Northland District Health Board
}

Rebecca Barbara Hay

A thesis submitted in partial fulfilment of the requirements for the degree of Master of Health Research (Midwifery), Victoria University of Wellington, 2020 


\section{Abstract}

\section{Background}

Prelabour rupture of membranes at term (PROM) is a subject of interest to women and maternity care providers alike. Management of PROM varies internationally, and regionally within New Zealand, despite the presence of interprofessional consensus statements. Northland District Health Board (NDHB) policy differs from most maternity care facilities by enabling expectant management of labour for women at low risk of transmission of Group $\beta$ Streptococcus to their baby to extend to 96 hours from time of rupture of membranes. This study aimed to explore whether the NDHB policy was applied in practice and safely served the needs of women and babies in this DHB.

\section{Methods}

A retrospective quantitative clinical notes review was conducted of files in a one-year sample. The clinical notes of 123 women who had duration from ROM to birth at term of 18 hours or more were reviewed. Statistical comparisons using percentages, means and odds-ratios were made to a one-year sample of all other births at term at NDHB facilities, and with birth data from the New Zealand College of Midwives Clinical Outcomes Research Database (COMCORD).

\section{Findings}

Variables including demographic data, antenatal and intrapartum care given, time factors and outcomes themselves were reviewed. Few variables impacted outcomes within the PROM sample, though use of intravenous oxytocin was associated with increased epidural use and increased incidence of instrumental birth. Wāhine Māori ${ }^{1}$ had an increased incidence of vaginal births but some variables and outcomes highlighted inequities, including reduced antenatal screening, a higher incidence of maternal smoking, severe postpartum haemorrhage, and admission to Special Care Baby Units. Duration splits at 48 and 96 hours 
were applied to identify whether time increased risks for women or babies, but duration did not appear to be a factor which increased risk. The phenomenon of PROM itself increased risk for women and babies, increasing rates of labour induction, augmentation, epidural use, operative births, postpartum haemorrhage, admission to Special Care Baby Units and decreased exclusive breastfeeding at discharge.

\section{Conclusions}

The NDHB PROM policy appeared to be applied consistently and did not appear to increase risk for women and babies. Risks were increased for all women with PROM, with some inequitable outcomes for wahine Māori and pēpi ${ }^{2}$. This research provides a comparison which is informative for clinical practice, education, and future research, and supports women's involvement in decision-making. 


\section{Acknowledgements}

There are many people who deserve thanks for their assistance and support while I completed this thesis. At Victoria University of Wellington, I would like to express my special thanks to my supervisors, Dr Robyn Maude and Dr Sue Calvert, for their undying interest and support. Dr Lisa Woods provided for statistical support, and Justin Cargill provided library and Endnote support. Graduate Women Wellington provided a grant which covered the practical costs, and Health Workforce New Zealand via the New Zealand College of Midwives covered the academic costs of this study.

At Northland District Health Board, my thanks go to Deb Pittam, Sue Bree, Dr David Bailey, Wendy Taylor and Denise McCormack for support and encouragement to complete this research. Special thanks to Tracey Hildreth in Release of Information, and data analyst Shane Stanners. Thanks also to Northland District Health Board for overall support of this project and for giving consent to use their organisational name in this thesis (see Appendix 1).

Lesley Dixon at NZCOM provided the COMCORD data. Midwifery and Quality leaders at all 20 DHBs in New Zealand lent their support by providing PROM and GBS policies, and midwives everywhere have been interested and supportive, not least Dr Celia Grigg. My workmates and manager Dr Katie Groom have been constant supporters.

My husband David, mum Susan, and children Marina, Olivia and Matthew have put up with it and been slightly impressed occasionally. 


\section{Contents}

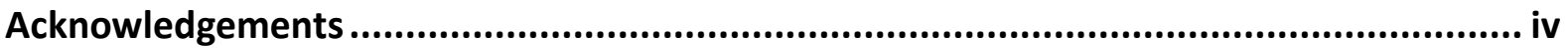

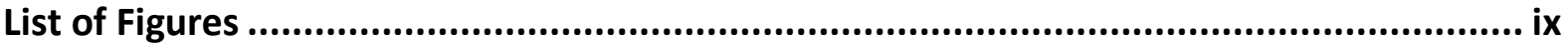

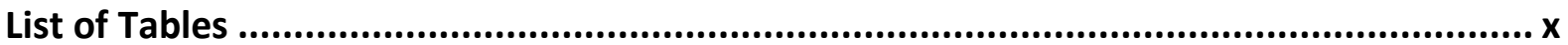

Abbreviations used in this thesis....................................................................................

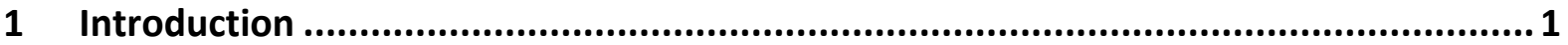

1.1 Personal experience: my background and interest in this topic .............................

1.2 NDHB Population/Demographic Data..................................................................

1.3 Maternity services in NDHB and Clinical Indicators ..............................................

1.1 Accurately identifying Spontaneous Rupture of Membranes ................................. 7

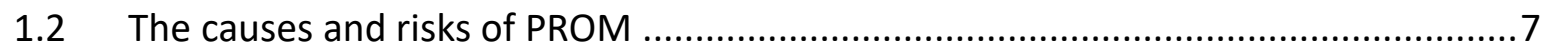

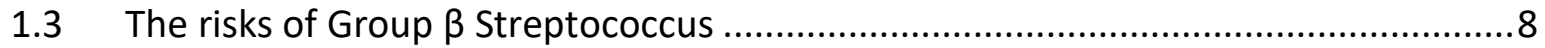

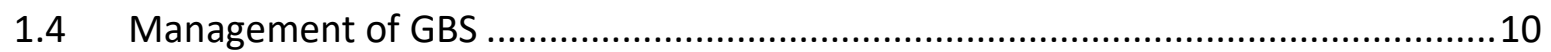

1.5 The medico-legal context for this study........................................................... 11

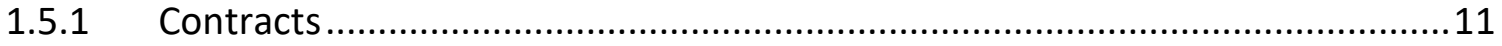

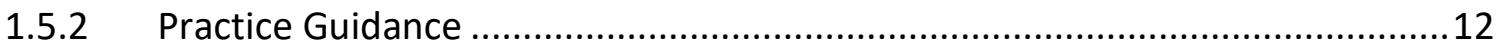

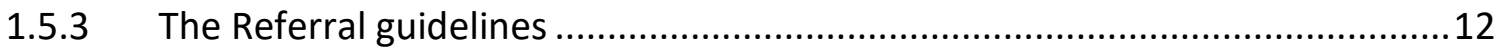

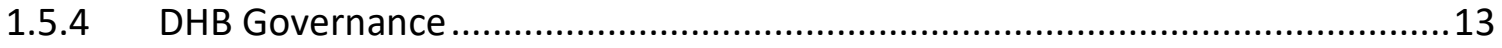

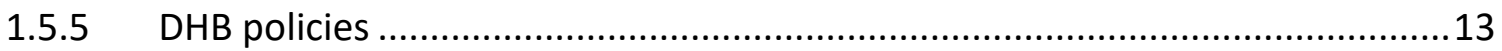

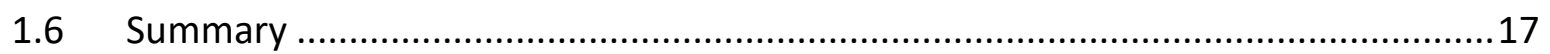

1.7 Research aim, questions, and objectives .......................................................

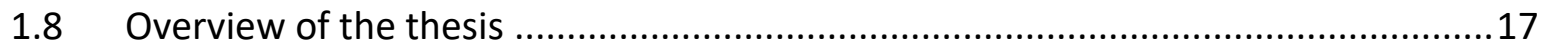

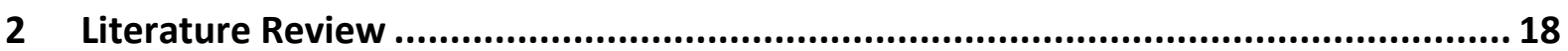

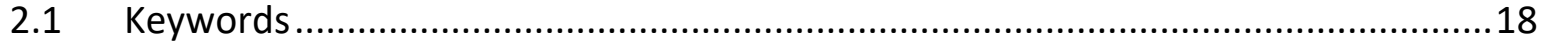

2.2 The aim and objectives of the literature review ................................................. 18

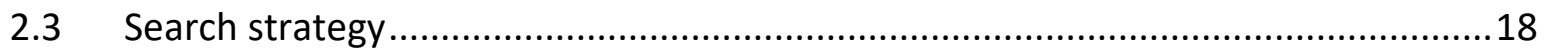

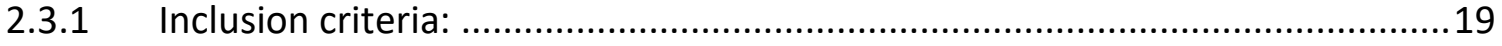

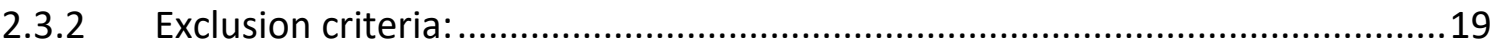

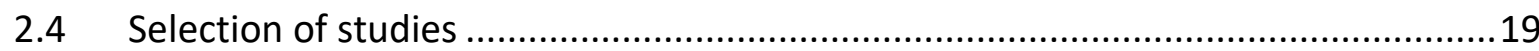

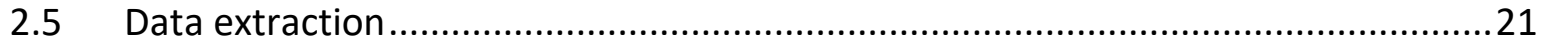

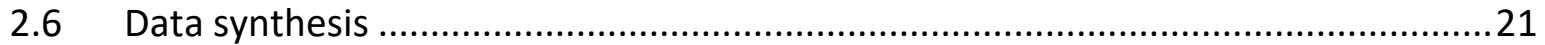

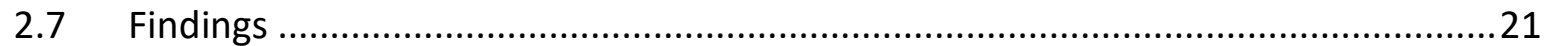




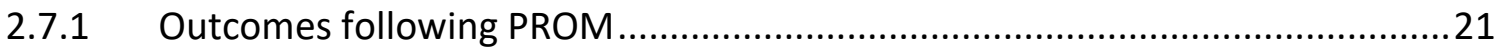

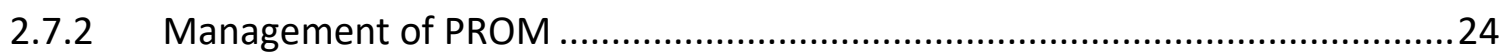

2.7.3 Methods of induction of labour following PROM .........................................28

2.7.4 Intravenous antibiotic prophylaxis following PROM .....................................31

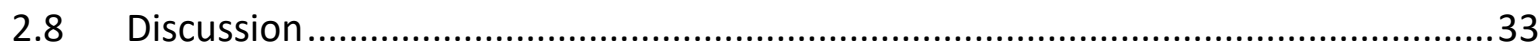

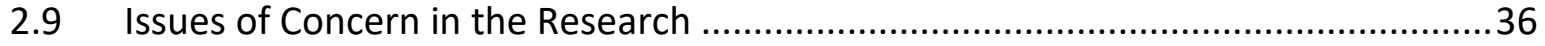

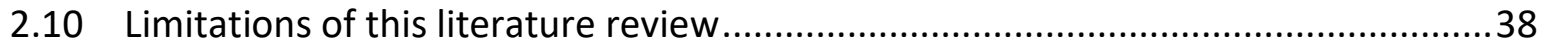

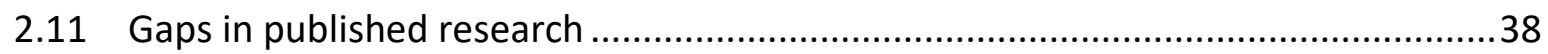

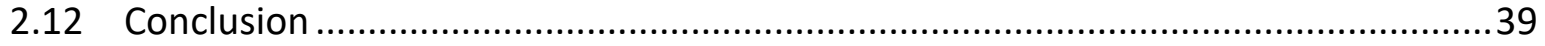

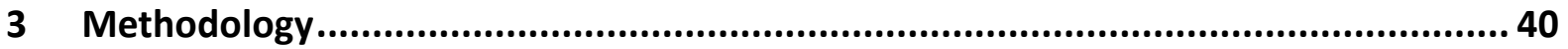

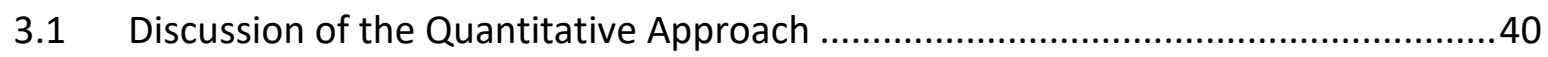

3.2 Methodology: Evaluation Research ................................................................ 41

3.3 Method: Clinical records review and database comparison .................................43

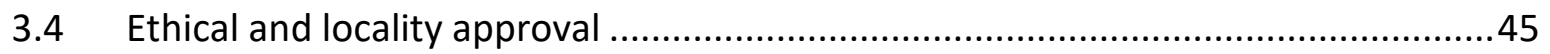

3.5 Treaty of Waitangi issues and Māori consultation ................................................46

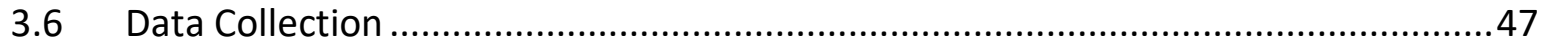

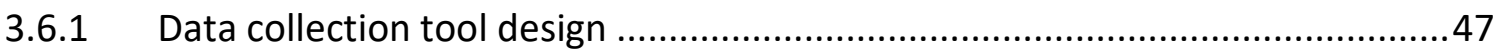

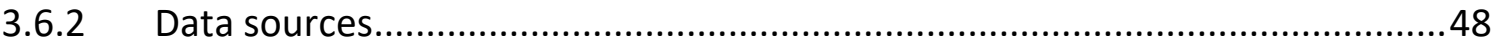

3.6.3 Clinical Data Recording and Data Entry ..................................................... 48

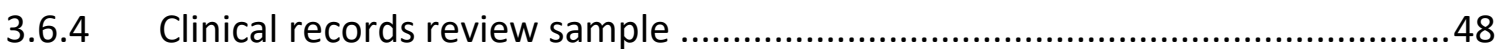

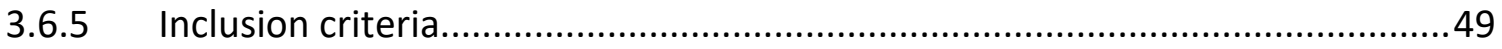

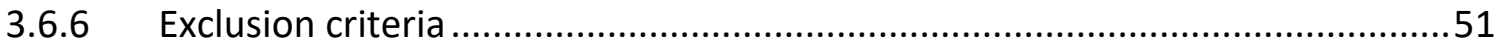

3.6.7 Issues arising during data collection and analysis ............................................52

3.6.8 Carrying out the clinical records review .......................................................53

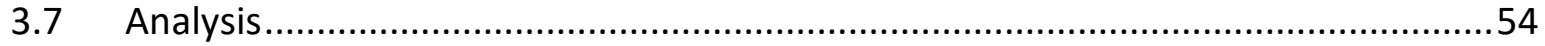

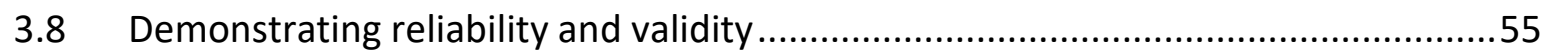

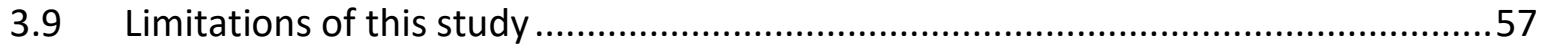

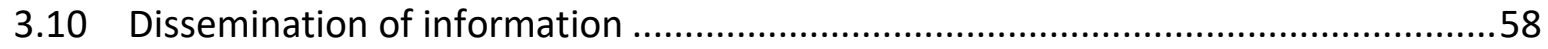

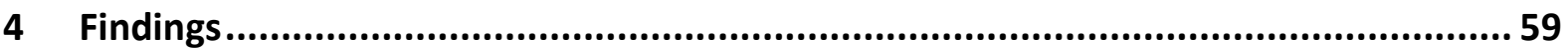

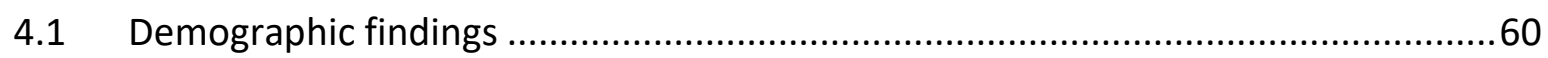

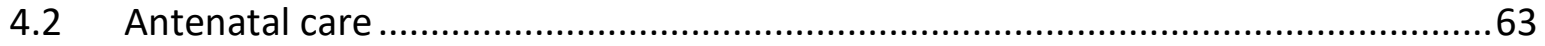

4.2.1 Registration with LMC by 12 weeks gestation.............................................63 


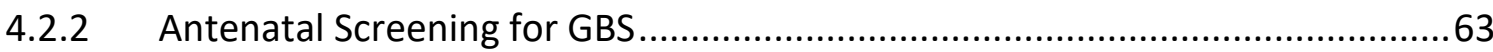

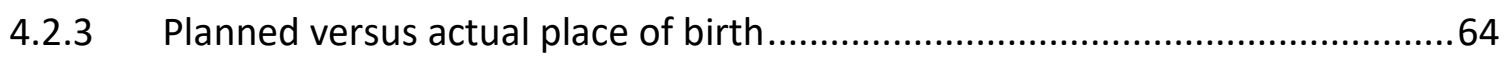

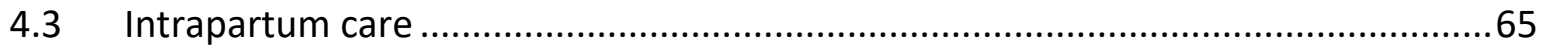

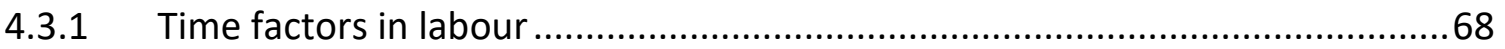

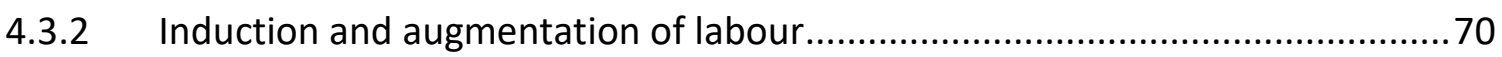

4.3.3 Maternal and fetal condition in labour.......................................................... 72

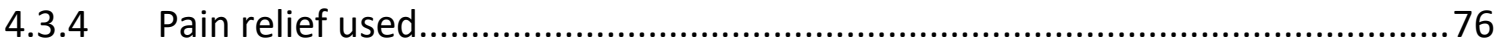

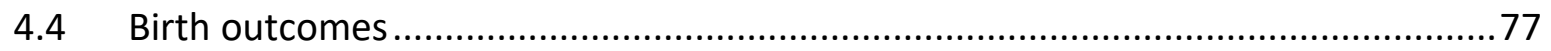

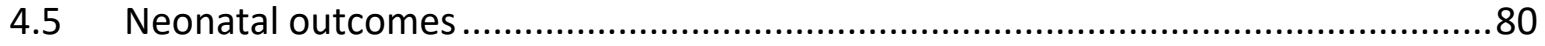

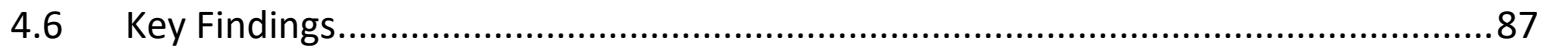

4.6.1 Māori vs non-Māori Outcomes......................................................................... 88

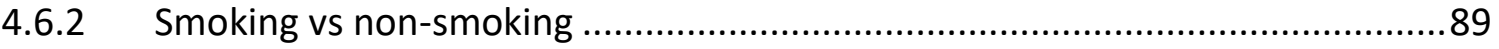

4.6.3 Impact of engagement of care following ROM............................................90

4.6.4 Did increasing duration of ROM to birth affect outcomes? .............................91

4.6.5 Did an artificial rupture of the membranes affect outcomes? ..........................94

4.6.6 Did oxytocin use in labour affect outcomes? ..............................................95

4.6.7 Did neonates who were small for gestational age have different outcomes?..96

4.6.8 Outcomes for women with PROM as a factor in their labour .........................97

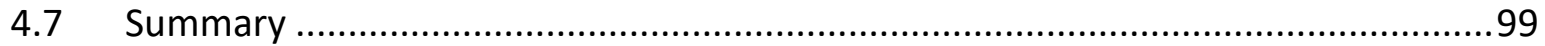

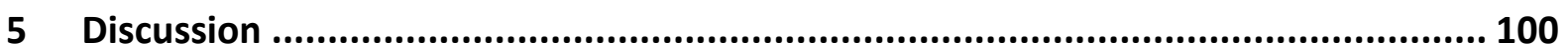

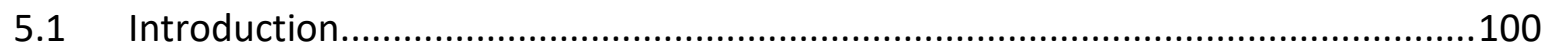

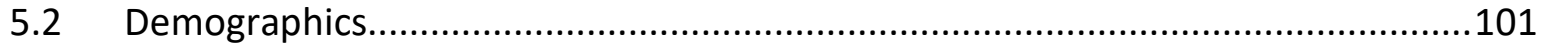

5.3 Antenatal Care

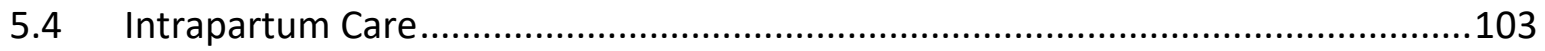

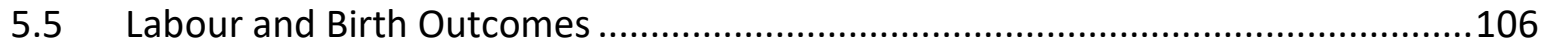

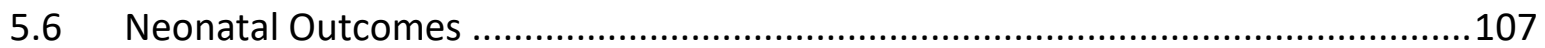

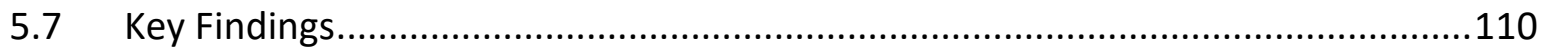

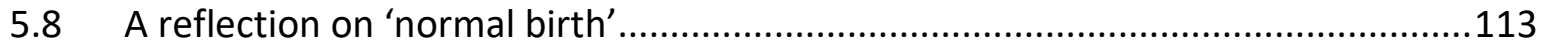

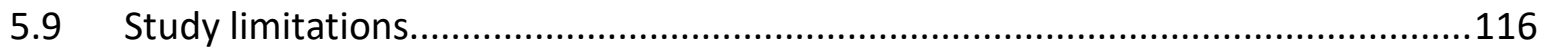

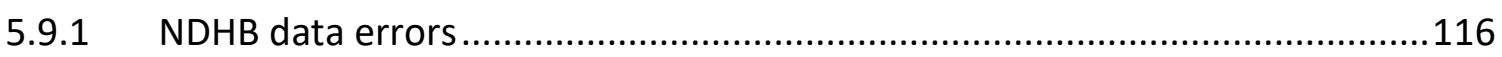

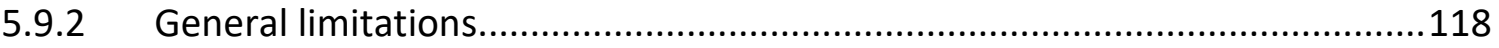

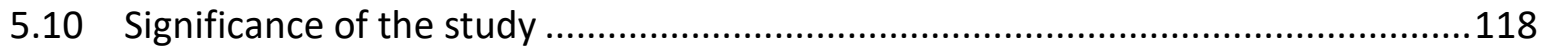


5.11 Recommendations for practice, education, and research

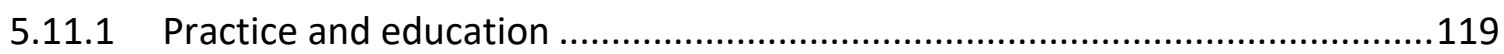

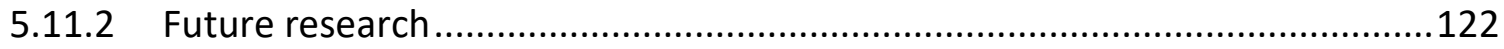

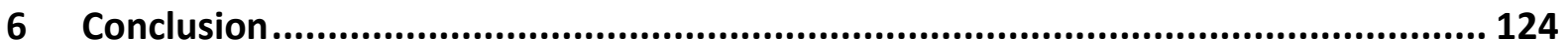

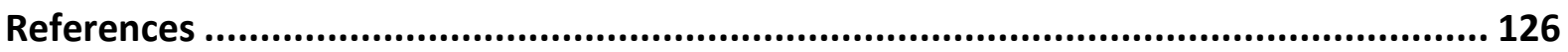

Appendix 1: Letter of introduction and confirmation of permission to identify NDHB in

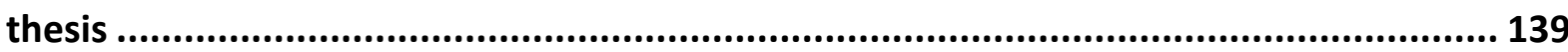

Appendix 2: New Zealand DHB PROM and GBS Policy References .............................. 140

Appendix 3: Health and Disability Ethics Committee response. ................................... 142

Appendix 4: Victoria University of Wellington Human Ethics Committee response ........ 143

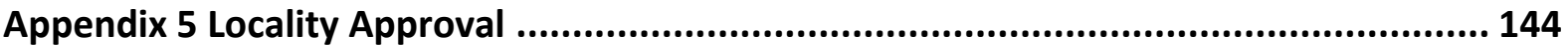

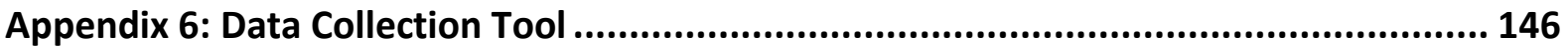

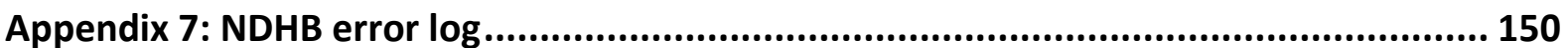




\section{List of Figures}

Figure 1-1 Northland deprivation deciles by population. ........................................................................... 3

Figure 1-2 Travel times across Northland and to Auckland......................................................................... 4

Figure 1-3 Northland term babies requiring respiratory support (\%). ............................................................ 7

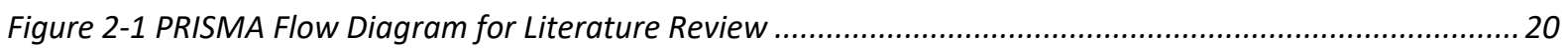

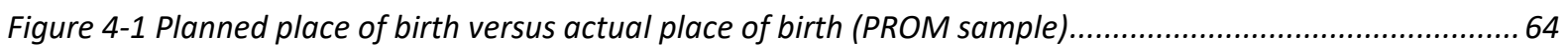

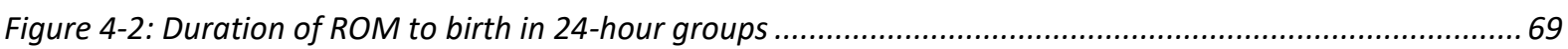

Figure 4-3: Clustered bar count of number of vaginal examinations given during labour care, by parity ........... 75

Figure 4-4 PROM sample duration of ROM to birth (hours) by neonatal weight $(\mathrm{g})$........................................ 82

Figure 4-5: NDHB duration of ROM to birth (hours) by neonatal weight ....................................................... 82

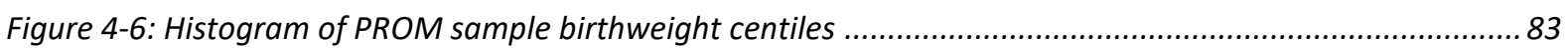

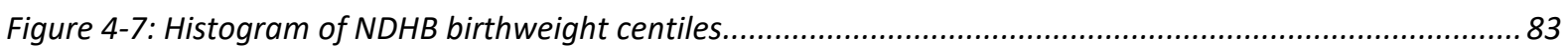

Figure 5-1 Clinical Indicator 20 (Ministry of Health, 2018, p. 79) ............................................................. 109

Figure 5-2 Flow diagram of elimination of non-normal birth features ........................................................ 114 


\section{List of Tables}

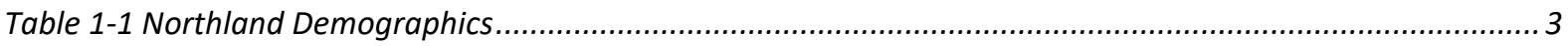

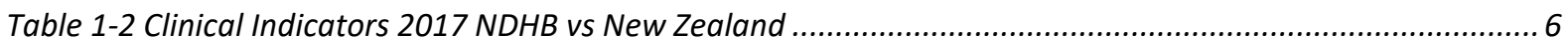

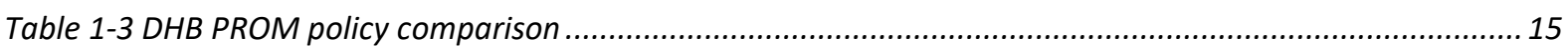

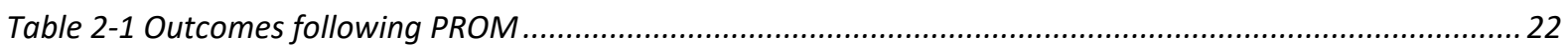

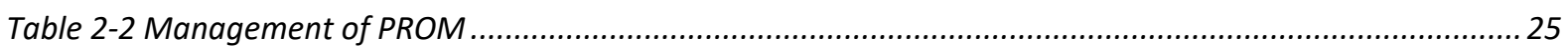

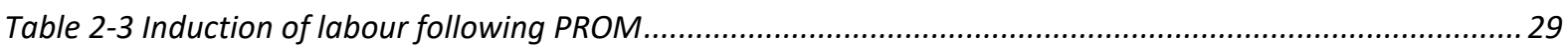

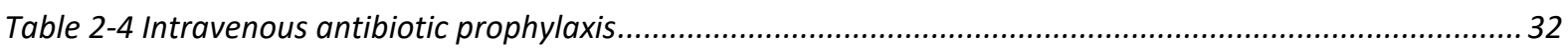

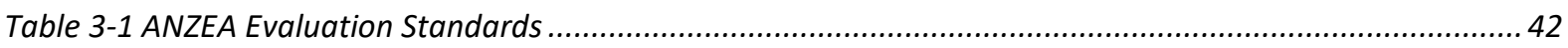

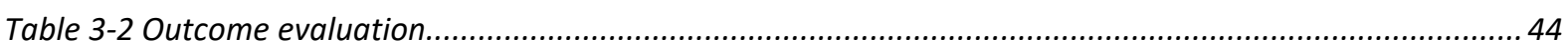

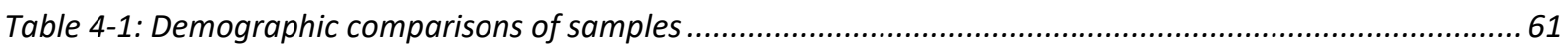

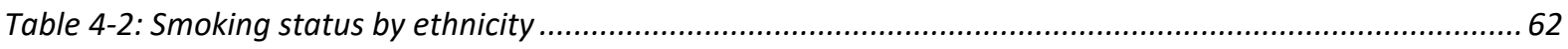

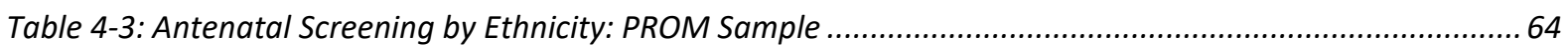

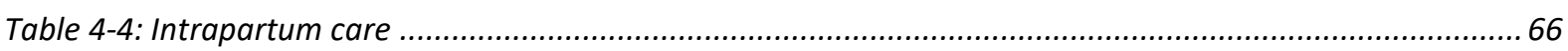

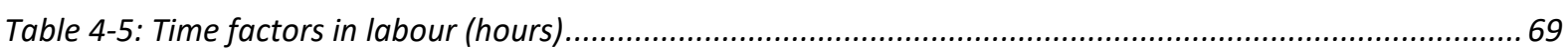

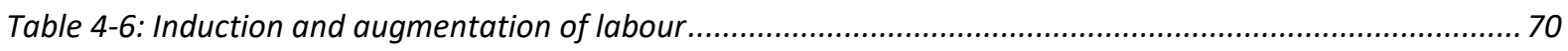

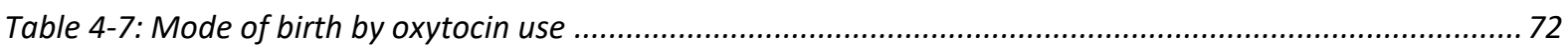

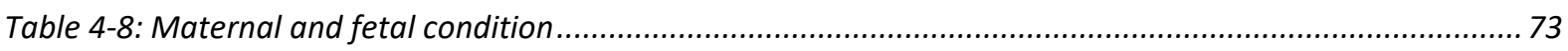

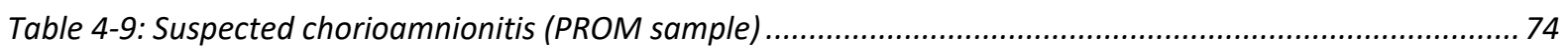

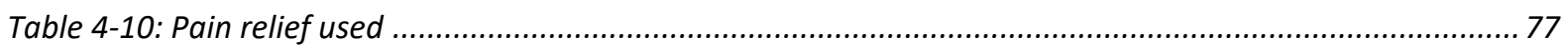

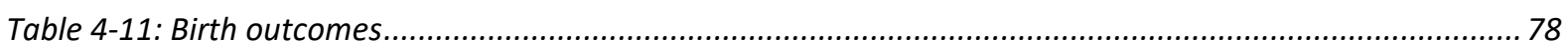

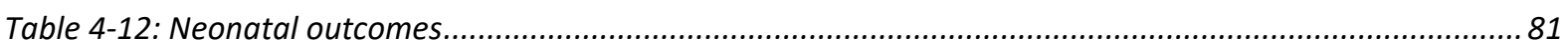

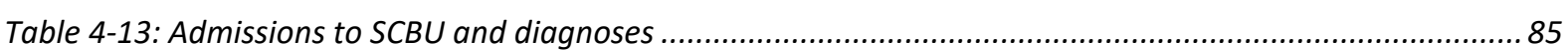

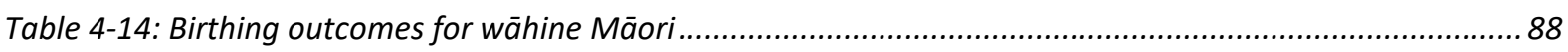

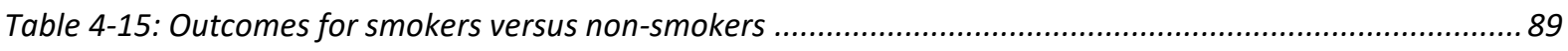

Table 4-16: Outcomes based on engagement with care ............................................................................90

Table 4-18: Outcomes by duration from ROM to birth .......................................................................... 91

Table 4-18: Outcomes for women with under or over 48 hours duration from ROM to birth............................92

Table 4-19: Outcomes for women with duration of under or over 96 hours from ROM to birth .........................93

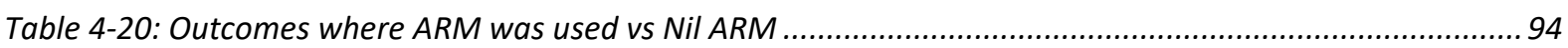

Table 4-21: Outcomes by oxytocin use versus nil oxytocin use ....................................................................95

Table 4-22: Outcomes for small for gestational age babies ........................................................................96

Table 4-23: PROM outcome comparisons vs NDHB outcomes .......................................................................97

Table 4-24: PROM outcomes comparisons for COMCORD sample of PROM $\geq 18 \mathrm{hrs}$ with no induction of labour 98 


\section{Abbreviations used in this thesis}

ARM

Amniotomy or artificial rupture of membranes, prior to or during labour, with the intention of inducing or augmenting labour

COMCORD New Zealand College of Midwives Care and Outcomes Research Database

DHB District Health Board

EOGBS Early-onset group B Streptococcus sepsis, caused by transmission of GBS from mother to neonate, within the first seven days of life

GBS $\quad$ Group $\beta$ Streptococcus

IAP Intravenous antibiotic prophylaxis

IOL Induction of labour

IV Intravenous

$\mathrm{MOH} \quad$ Ministry of Health

NDHB Northland District Health Board

NHI National Health Index number

NICE National Institute for Health and Care Excellence

NICU Neonatal Intensive Care Unit

NZCOM New Zealand College of Midwives

PPROM Preterm prelabour rupture of membranes ( $\leq 36+6$ weeks), prior to the onset of labour 
PROM

Prelabour (or premature) rupture of membranes at term ( $\geq 37$ weeks), prior

to the onset of labour ${ }^{3}$

RANZCOG Royal Australian and New Zealand College of Obstetricians and

Gynaecologists

ROM

Rupture of membranes, whether spontaneous or artificial

SCBU

Special Care Baby Unit

SRM

Spontaneous rupture of fetal membranes, prior to or during labour

(Chan et al., 2014; Middleton, Shepherd, Flenady, McBain, \& Crowther, 2017; Wise,

Campbell, \& Darlow, 2015)

\footnotetext{
${ }^{3}$ Prelabour rupture of membranes is also known as premature rupture of membranes, and the search for international literature located more results when using the term 'premature' than 'prelabour'. 'Prelabour' is most used in New Zealand, and is the term used in the most recent Cochrane review.
} 


\section{Introduction}

Prelabour rupture of membranes at term ${ }^{4}$ (PROM) occurs in approximately $8 \%$ of women. Most women (60\%) experiencing PROM will commence labour within 24 hours, and 95\% of women will commence labour within 72 hours (Grant \& Keirse, 1989; Gunn, Mishell, \& Morton, 1970). The primary concern following PROM is the risk of ascending infection, particularly of group $\beta$ Streptococcus (GBS), causing maternal or neonatal morbidity or mortality during the intrapartum or postpartum period (Middleton, Shepherd, Flenady, McBain, \& Crowther, 2017). A typical time-frame to await the spontaneous onset of labour after PROM is 24 hours, however at Northland District Health Board (NDHB) women experiencing spontaneous rupture of membranes (ROM) may be offered a period of up to 96 hours to wait for labour to establish naturally. This thesis discusses the NDHB approach and considers the outcomes arising from it.

This introductory chapter explores the background to this evaluation study, which is focused within the NDHB catchment area. The demography of Northland and local maternity services are described. The identification and risks of PROM, and of GBS are detailed. The New Zealand medico-legal context for maternity care provision is described, practice guidelines are outlined, and variations between District Health Board (DHB) policies from around New Zealand relevant to PROM and GBS management are explored. Key literature of influence in New Zealand policy writing is examined and contrasted with the NDHB PROM policy. An overview of the thesis completes this chapter and introduces the methodology chapter.

\subsection{Personal experience: my background and interest in this topic}

I commenced midwifery practice as a Lead Maternity Carer (LMC) midwife based in Warkworth, providing maternity care for women in a rural and remote rural area, primarily in Waitemata DHB, and some within NDHB. During LMC practice and later as a core midwife at 
Introduction

North Shore Hospital (within Waitemata DHB), I had experienced caring for many women with PROM.

I attended a birth conducted by a colleague referencing the NDHB policy, where the woman elected expectant management, and birthed quickly and in good health at home, four days after PROM had occurred. I also cared for women birthing in hospital in Waitemata DHB, who chose or were recommended to have active management following PROM. Often these women experienced challenging or difficult labours and needed oxytocin infusions to stimulate labour, epidural anaesthesia, and sometimes a further artificial rupture of membranes to progress labour. Some women with low risk factors and ROM of 18-24 hours would choose to spend a night at a primary birthing centre with intravenous antibiotic prophylaxis (IAP). Sometimes this worked well, and the woman would establish into labour and birth spontaneously at the primary centre, and sometimes it was just one stage in a long process involving transfer to a secondary hospital and active management. Working with two differing policies caused me a degree of cognitive dissonance and raised questions of equity. These factors led to my long-standing interest in the topic of PROM, and my desire to explore how PROM is managed at NDHB. This interest has developed further since working at the Liggins Institute of the University of Auckland, where a research focus is protecting integrity of the maternal-infant microbiome. I also serve on the Massey University Human Ethics Committee (Northern), which kindles my interest in ethics, medico-legal practices, and consumer choices.

\subsection{NDHB Population/Demographic Data}

Data from the 2018 Census (Stats New Zealand, 2020) gives Northland a population of 179,076 people, or $3.7 \%$ of the total New Zealand population. Ethnicity is less diverse in Northland than in wider New Zealand, and Māori people are more prevalent, as shown in Table 1-1. The 2018 NDHB Maternity Quality and Safety publication also reports women birthing at NDHB facilities by ethnicity, with $45.8 \%$ Māori, $46.5 \%$ New Zealand European, Asian 5.62\%, and others 2.03\% (NDHB, 2018a).

The median age for Northlanders is greater than for the national average, though for Māori Northlanders the median age is younger, reflecting both a shorter life expectancy and a 
Introduction

higher fertility rate. Qualification levels and engagement in employment are lower in Northland than across New Zealand. Incomes are also lower in Northland, with a clear divide between Māori and non-Māori (Stats NZ, 2020).

Table 1-1 Northland Demographics

\begin{tabular}{lrrrr} 
& Northland & New Zealand & $\begin{array}{r}\text { Northland } \\
\text { Māori }\end{array}$ & $\begin{array}{r}\text { New } \\
\text { Zealand } \\
\text { Māori }\end{array}$ \\
\hline Ethnicity' & & & & \\
$\quad$ European & $73.1 \%$ & $70.2 \%$ & & \\
$\quad$ Māori & $36.0 \%$ & $16.5 \%$ & & \\
$\quad$ Pasifika & $4.2 \%$ & $8.1 \%$ & & \\
$\quad$ Asian & $3.9 \%$ & $15.1 \%$ & & \\
$\quad$ Other & $1.7 \%$ & $2.7 \%$ & & \\
Median age & 42.6 & 37.4 & 27.2 & \\
Total Qualifications (aged 15+) & & & & \\
$\quad$ Any formal qualification & $76.9 \%$ & $81.8 \%$ & $71.8 \%$ & $74.7 \%$ \\
$\quad$ Bachelor's degree or higher & $15.8 \%$ & $24.8 \%$ & $9.6 \%$ & $12.5 \%$ \\
Unemployment (aged 15+) & $5.2 \%$ & $4.0 \%$ & $10.0 \%$ & $8.1 \%$ \\
Median income & $\$ 24,800$ & $\$ 31,800$ & & \\
\hline
\end{tabular}

1 People may have stated more than one ethnic group

Northland has a depth of deprivation (see

Figure 1-1) paralleled in New Zealand only in the eastern Bay of Plenty and Tairawhiti. The New Zealand Deprivation Index ranks a score of deprivation from 1-10, with people residing in an area scoring 1 being the least deprived 10\% of New Zealand, and people residing in an area scoring 10 within the most deprived $10 \%$ of the country. The score is calculated based on household incomes, home ownership, support, employment, qualifications, living space, access to communication and transport (White, Gunston, Salmond, Atkinson, \& Crampton, 2008).
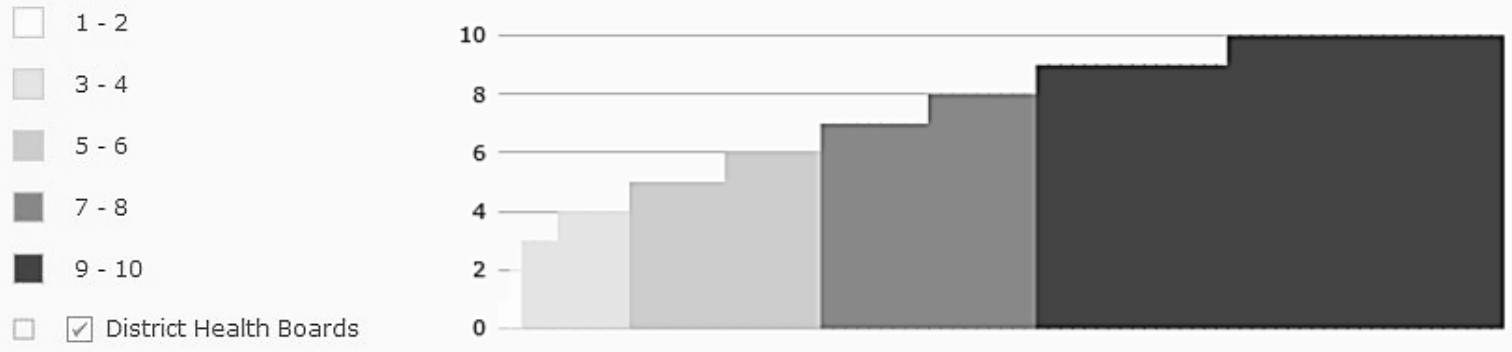

Figure 1-1 Northland deprivation deciles by population. 
Introduction

Adapted from Healthspace: New Zealand Index of Deprivation, Massey University, 2015.

Aspects of deprivation are further pronounced between Māori and non-Māori, although the region as a whole faces challenges which impede access to health services (NDHB, 2018a). Distances and travel times between medical and other services can be great (see Figure 1-2).

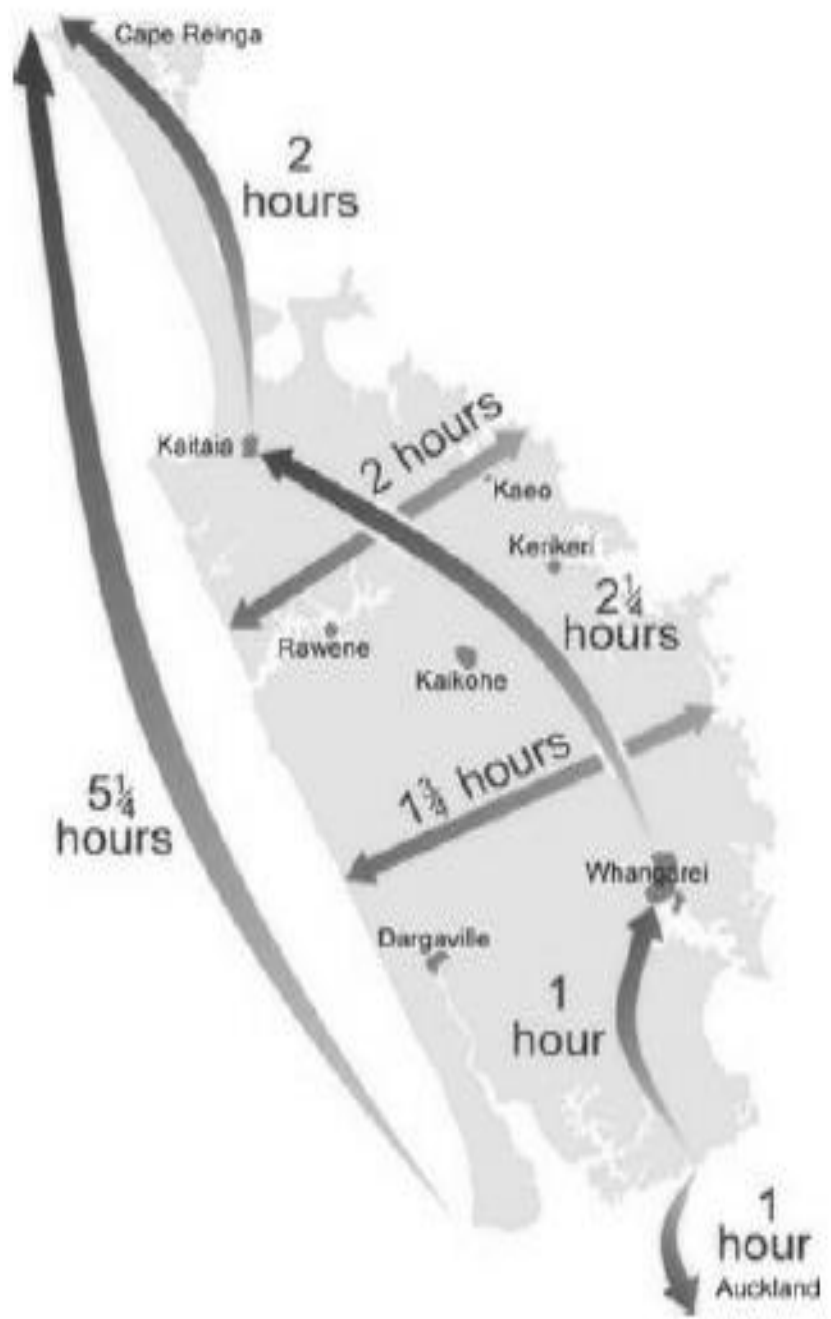

Figure 1-2 Travel times across Northland and to Auckland.

Adapted from: 2018 Maternity Quality and Safety Annual Report, NDHB, 2018.

\subsection{Maternity services in NDHB and Clinical Indicators}

Maternity services provided by NDHB are anchored at Te Kotuku, a secondary maternity service within Whangarei Base Hospital, which hosts $77 \%$ of births within NDHB. Secondary 
Introduction

services include obstetric, anaesthetic, medical and surgical support, and a Special Care Baby Unit (SCBU) for neonates from 32 weeks gestation. Primary units, which host $16.4 \%$ of NDHB births, are based within DHB hospital facilities at Kawakawa (Bay of Islands) and Kaitaia. Dargaville Hospital provides antenatal and postnatal midwifery services only (Healthpoint Ltd, n.d.; NDHB, 2018a). Excluded from this study for practicality reasons are the $4.6 \%$ of births occurring at home, and the 30-50 annual births at the primary birthing unit operated in Rawene by the Hokianga Health Enterprise Trust (Hokianga Health Enterprise Trust, 2018; Ministry of Health, 2018b). Women in NDHB largely access maternity care from LMC and DHB midwives. Publicly-funded obstetric consultation is available at Te Kotuku, and Kaitaia and Bay of Islands have a weekly obstetric consultation service (NDHB, 2018a).

New Zealand Maternity Clinical Indicators 2017 reports several interesting differences between NDHB and wider New Zealand data for standard primiparae (see Table 2). Vaginal births were more common, and instrumental and caesarean births less common within NDHB compared to all New Zealand (Ministry of Health, 2019b). Induction of labour was relatively low for standard primiparae. The vaginal birth rate for standard primiparae at Te Kotuku for 2016 fell from $74.5 \%$ to $69.7 \%$ and this drop was associated with an increase of Indian and Asian women birthing there (NDHB, 2018a).

Other differences seen in Table 1-2 between local and national outcomes for standard primiparae ${ }^{5}$ reflect positively on NDHB. NDHB women had a high rate of intact lower genital tract following birth, and episiotomies were used less frequently. General anaesthesia was used for caesarean birth more frequently than on average, however NDHB propose this is because most of their caesareans are truly emergency procedures, and it is representative of a similar pattern at DHBs with low rates of caesarean births (NDHB, 2017; Ministry of Health, 2019b).

The rate of preterm birth was lower than the national average and the reason for this is not known, although NDHB propose continuity of care may be a factor. NDHB is focusing on

\footnotetext{
5 a woman aged 20-34 birthing for the first time and at term, "expected to have an uncomplicated pregnancy" (Ministry of Health, 2019b, p. 8)
} 
Introduction

smoking cessation programmes due to a high rate of maternal smoking during pregnancy and after birth. NDHB consistently had a high rate of small for gestational age babies born at term and post-dates, and attributes this to the high rate of smoking. There has been some reduction in the number of small for gestational age babies between the 2016 and 2017 Quality and Safety Reports (Ministry of Health, 2019b; NDHB; 2017, 2018a).

Table 1-2 Clinical Indicators 2017 NDHB vs New Zealand

\begin{tabular}{|c|c|c|c|}
\hline Number & Indicator Category & Northland & New Zealand \\
\hline 1 & Women registered with an LMC in first trimester & $65.3 \%$ & $72.3 \%$ \\
\hline \multicolumn{4}{|c|}{ Standard primiparae having: } \\
\hline 2 & Spontaneous vaginal birth & $73.5 \%$ & $65.1 \%$ \\
\hline 3 & instrumental vaginal birth & $12.7 \%$ & $16.3 \%$ \\
\hline 4 & caesarean birth & $12.3 \%$ & $17.6 \%$ \\
\hline 5 & induction of labour & $3.9 \%$ & $7.6 \%$ \\
\hline 6 & intact lower vaginal tract & $41.2 \%$ & $27.7 \%$ \\
\hline 7 & episiotomy with no 3rd or 4th degree tear & $14.1 \%$ & $24.5 \%$ \\
\hline 8 & $3 r d$ or 4 th degree tear with no episiotomy & $3.8 \%$ & $4.4 \%$ \\
\hline 9 & episiotomy with 3rd or 4th degree tear & $2.1 \%$ & $1.7 \%$ \\
\hline \multicolumn{4}{|c|}{ Women giving birth, having: } \\
\hline 10 & general anaesthetic for caesarean birth & $13.0 \%$ & $8.2 \%$ \\
\hline 11 & blood transfusion with caesarean birth & $3.5 \%$ & $3.1 \%$ \\
\hline 12 & blood transfusion with vaginal birth & $1.4 \%$ & $2.2 \%$ \\
\hline 13 & diagnosed eclampsia at birth admission & 0 & 17 \\
\hline 14 & peripartum hysterectomy & 1 & 29 \\
\hline 15 & $\begin{array}{l}\text { admission to Intensive Care Unit and requiring } \\
\text { ventilation during the pregnancy or postnatal } \\
\text { period }\end{array}$ & 0 & 11 \\
\hline 16 & maternal tobacco use during postnatal period & $22.2 \%$ & $10.5 \%$ \\
\hline \multicolumn{4}{|c|}{ Live born babies: } \\
\hline 17 & preterm birth (under 37 weeks gestation) & $6.7 \%$ & $7.5 \%$ \\
\hline 18 & $\begin{array}{l}\text { small babies at term } 37-42 \text { week, under the } 10 \text { th } \\
\text { centile for their weight }\end{array}$ & $2.3 \%$ & $2.9 \%$ \\
\hline 19 & $\begin{array}{l}\text { small babies at term born at } 40-42 \text { weeks } \\
\text { gestation }\end{array}$ & $35.4 \%$ & $31.9 \%$ \\
\hline 20 & $\begin{array}{l}\text { babies born at } 37+\text { weeks gestation requiring } \\
\text { respiratory support for } 4 \text { hours or more }\end{array}$ & $3.6 \%$ & $2.0 \%$ \\
\hline
\end{tabular}

NDHB had the highest rate nationally of term neonates admitted to SCBU for more than four hours respiratory support. This difference is consistent between Māori and European babies born in NDHB, (see Figure 1-3). NDHB reports the high numbers of neonates requiring respiratory support is reducing following a focused intervention programme, audit 
Introduction

of neonatal admissions, and a new paediatrician changing established practices (Ministry of Health, 2019b; NDHB, 2018a).

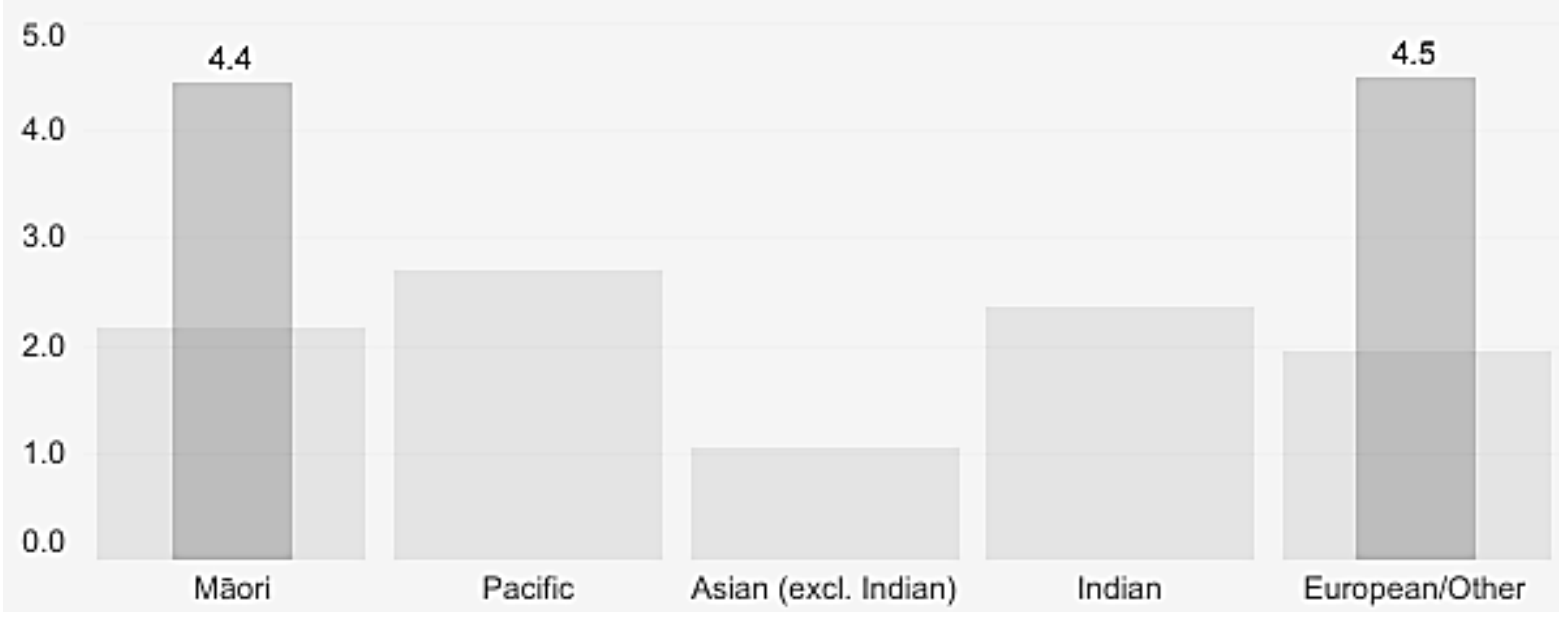

Key: NDHB in dark grey, New Zealand in light grey.

Figure 1-3 Northland term babies requiring respiratory support (\%).

Adapted from: Atlas of healthcare variation: Maternity. Health Quality and Safety Commission New Zealand, 2018.

\subsection{Accurately identifying Spontaneous Rupture of Membranes}

Ruptured membranes can be difficult to diagnose, as up to $25 \%$ of women present with no clinical signs of rupture other than verbal history (El-Messidi \& Cameron, 2010). Clinical skills used to diagnose ruptured membranes include taking an accurate and complete history, observing liquor draining, or visualisation of liquor via a speculum examination. Some units employ further diagnostic tools including litmus-based nitrazine swabs, Amnisure, a point of care test produced by Qiagen Sciences (2019), ultrasound scanning or vaginal secretion ferning test. Digital examination is discouraged (NICE, 2014; RANZCOG, 2017).

\subsection{The causes and risks of PROM}

For individual women, it is typically unknown why membranes rupture prior to labour.

Preventative measures are unknown, though selenium supplementation in pregnancy has 
Introduction

been proposed (Tara et al., 2010). PROM may be related to polyhydramnios ${ }^{6}$, maternal smoking, or mechanical rupture of the chorioamniotic membranes through physiological weakening. Alternatively, penetrative interventions such as vaginal examination, sex or infection are proposed as causative factors (El-Messidi \& Cameron, 2010). Membrane granulosis caused by chemical signalling via increased levels of cytokines/chemokines and chemotaxis in the amniotic membranes is proposed as another cause of PROM (GomezLopez, Hernandez-Santiago, Lobb, Olson, \& Vadillo-Ortega, 2013). Cellular changes to amniotic membranes are also proposed, attributed to high mean ambient temperatures causing increased incidence of PROM by Song et al. (2019), though this effect was most clearly seen at a mean ambient temperature of $32^{\circ} \mathrm{C}$ (RR 2.161,95\% $\mathrm{Cl}$ [1.240-3.764]), which is in excess of typical mean ambient temperatures in New Zealand. The cause of PROM may be idiopathic; however, PROM creates risks for both mother and baby.

Immediate risks following any ROM include cord accidents such as prolapse or compression, or placental abruption (Middleton et al., 2017). ROM in the absence of uterine contractions or with a low Bishops Score ${ }^{7}$ is associated with prolonged duration to established labour (Hiersch et al., 2017). Interventions may be introduced, such as induction of labour, which may be considered an additional risk of PROM as it may trigger subsequent interventions. PROM is also associated with increased risk of caesarean or instrumental birth. Maternal and neonatal infection are the main risks of PROM, with risk of sepsis increasing with duration from the time of membrane rupture to the time of birth (Middleton et al., 2017; RANZCOG, 2017). GBS was first diagnosed as causing sepsis in a neonate in 1964 (Eickhoff, Klein, Daly, Ingall, \& Finland, 1964), and is the primary causative organism in early-onset sepsis at term in New Zealand and Australia (Singh, Barnes, \& Isaacs, 2019).

\subsection{The risks of Group $\beta$ Streptococcus}

GBS is a transient common organism which may colonise the gastrointestinal tract, urethra, or vaginal tract. Colonisation rates in women vary internationally (Ohlsson \& Shah, 2014),

\footnotetext{
${ }^{6}$ Excessive level of amniotic fluid (Dare, Middleton, Crowther, Flenady, \& Varatharaju, 2006)

${ }^{7}$ Assessment tool used to evaluate the condition of the cervix, including dilatation, effacement, consistency, position of the cervix and station of the presenting fetal part (Bishop, 1964).
} 
Introduction

and Grimwood et al. (2002) found it to be present in $22 \%$ of New Zealand women in late pregnancy. GBS is highly sensitive to penicillin and vancomycin and moderately sensitive to erythromycin (LabPlus, 2018), however treatment does not prevent subsequent recolonisation. GBS colonisation is typically asymptomatic, though may cause urinary tract infection or wound infections. Ascending infection of GBS can lead to chorioamnionitis in women before or during labour, endometritis postnatally, or rarely, meningitis (Chan et al., 2014; Melin, 2011; Middleton et al., 2017). Many cases of maternal pyrexia or suspected chorioamnionitis never have any organism identified as the cause and do not go on to cause neonatal morbidity (Towers et al., 2017). Ismail and Lahiri (2013) state chorioamnionitis occurs in $8-10 \%$ of women with PROM, rising to $40 \%$ of women with prolonged PROM (>24 hours). If accurate, this incidence should be found in the one-year sample included in this evaluation.

GBS is the organism responsible for causing most cases of neonatal sepsis at term in the developed world, with associated significant morbidity and mortality (Singh et al., 2019). As many as $70 \%$ of babies born to women with GBS will become colonised with GBS themselves. For the majority, GBS is not problematic, however PROM increases the risk of early-onset group $\beta$ Streptococcus (EOGBS) infection and subsequent illnesses (Palasanthiran, Starr, Jones, \& Giles, 2014). NICE (2014) state that for babies born following PROM at term, the risk of any serious infection is $1 \%$, twice that of babies born when membranes have ruptured during established labour. Increasing duration of time to birth and culture-confirmed chorioamnionitis increases neonatal risk (Ismail \& Lahiri, 2013). The incidence of neonatal EOGBS infection in New Zealand and Australia is most recently stated as 0.43 per 1000 live births (Singh et al., 2019). Neonatal illnesses caused by GBS includes pneumonia, septicaemia, meningitis or osteomyelitis. Neonates surviving meningitis caused by GBS have a risk of up to $50 \%$ of neurological impairment, hearing and/or vision loss (Melin, 2011).

Onset of EOGBS can be rapid, with $90 \%$ of cases presenting within the first 12 hours following birth, but identification of a causative organism can take 36-48 hours (Wise et al., 2015). Symptoms of EOGBS include "respiratory distress, apnoea, temperature instability, tachycardia, lethargy, poor feeding, shock or "unwell,"”' (Darlow et al., 2015, p. 73). Untreated neonatal sepsis of any kind has a mortality rate of up to $50 \%$, however with 
Introduction

antibiotic treatment, mortality is $1 \%$ (Ismail \& Lahiri, 2013). Antibiotic treatment is usually commenced based on clinical symptoms, with blood samples sent to the laboratory for culture. Typically, only about $50 \%$ or so of neonates suffering EOGBS had known risk factors (Chan et al., 2014).

Late onset of disease caused by GBS, generally in the form of bacteraemia or meningitis, occurs between one week to three months of age. Infection may be attributed to other sources in the community or hospital, including breastfeeding. It is not affected by intrapartum IAP administration (Melin, 2011), and is not a focus within this thesis.

\section{$1.4 \quad$ Management of GBS}

Management of maternal GBS status differs internationally, between universal screening or a risk-based approach. Universal screening of all women at $35-37$ weeks $^{8}$ via a low vaginal and perianal swab is recommended by the American Centers for Disease Control and Prevention (CDC), (2018) and in Canada by an interdisciplinary consensus guideline (Money \& Allen, 2016). A risk-based approach is recommended by the United Kingdom National Screening Committee, supported by the Royal College of Obstetricians and Gynaecologists (RCOG), (2016), who cite financial cost without benefit, and over-prescription of maternal antibiotics. The Royal Australian and New Zealand College of Obstetricians and Gynaecologists (RANZCOG) state that either approach is appropriate (2016), and hospitals in Australia elect their own approach (Singh et al., 2019). The New Zealand Consensus Guideline follows the RCOG risk-factor intrapartum management:

"The risk-based approach recommends that all women with one or more of the following factors be offered intravenous intrapartum antibiotic prophylaxis (IAP):

a) a previous GBS-infected baby

b) GBS bacteriuria of any count during the current pregnancy

c) preterm (<37 weeks) labour and imminent birth

d) intrapartum fever $>38^{\circ} \mathrm{C}$

\footnotetext{
${ }^{8}$ This has been recommended to change to $36-37^{+6}$ weeks by the American College of Obstetricians and Gynaecologists (American College of Obstetricians and Gynecologists, 2020)
} 
Introduction

e) membrane rupture $>18$ hours" (Darlow et al., 2015, p. 70).

If a woman has GBS found incidentally on a vaginal swab at any stage of pregnancy, it is recommended the swab is repeated at 35-37 weeks, or it is considered she has a risk factor for GBS (Darlow et al., 2015).

\subsection{The medico-legal context for this study}

This study considers the outcomes for women birthing within NDHB facilities, but nationally all care is given within the public health medico-legal framework under the Public Health and Disability Act, 2000, which established DHBs (Parliamentary Counsel Office, 2000). The Ministry of Health (MOH) administers the Act by regulating and funding DHBs ( $\mathrm{MOH}, 2018 \mathrm{a})$ as well as maternity service providers such as LMC midwives. The Health Practitioners Competence Assurance Act 2003 (Parliamentary Counsel Office, 2003) defines the role and purpose of the Medical Council of New Zealand, which regulates obstetricians (and other doctors) and similarly the Midwifery Council, which regulates midwives. RANZCOG and NZCOM provide professional practice guidance to their members.

\subsubsection{Contracts}

The $\mathrm{MOH}$ has a funding and advisory role, with the Primary Maternity Services Notice 2007 contracting the terms and conditions for payment of lead maternity carers providing primary maternity care. The $\mathrm{MOH}$ also contracts with DHBs to provide secondary and tertiary care $(\mathrm{MOH}, 2007)$. Referral between primary, secondary and tertiary services follows the framework provided through the Guidelines for Consultation with Obstetric and Related Medical Services (known as the Referral Guidelines), (MOH, 2012). The Referral Guidelines were written and reviewed by an Expert Working Group under the $\mathrm{MOH}$ umbrella. The $\mathrm{MOH}$ also produces many regular publications, including the New Zealand Maternity Clinical Indicators, National Maternity Monitoring Group Annual Reports, and other topical service reports $(\mathrm{MOH}, 2019 \mathrm{a})$. 
Introduction

\subsubsection{Practice Guidance}

Practice guidance comes from a multitude of stakeholders, including the $\mathrm{MOH}, \mathrm{RANZCOG}$, NZCOM and DHBs. Limited clinical guidance is provided by the National Maternity Monitoring Group which was created in 2012 to produce National Maternity Clinical Guidance documents (MOH, 2019a). RANZCOG and NZCOM both produce practice guidelines specific to maternity care, including consensus statements with interprofessional collaboration in some cases. PROM at term is the subject of a RANZCOG (2017) practice guide, and there is an interprofessional consensus guideline for the prevention of EOGBS, which is approved by NZCOM, RANZCOG, the Paediatric Society of New Zealand, and the Australasian Society of Infectious Diseases (Darlow et al., 2015).

New Zealand uses risk-based management of GBS, and if risk factors are present IAP is recommended, which requires women to remain at a maternity facility. Women with PROM but without risk factors for GBS are usually offered expectant management of labour for up to 18 to 24 hours and can monitor their wellbeing themselves with LMC midwifery support (Darlow et al., 2015). Women are advised to monitor symptoms including their temperature and vaginal discharge, should live/stay near to the hospital (not defined), have transport and support at home, and avoid sexual intercourse. Fetal movements and heart rate should be assessed at least once every 24 hours and the woman is advised to contact her midwife with any concerns about fetal movements. Induction of labour is recommended if women have or develop risk factors for GBS at any time following PROM (RANZCOG, 2017).

\subsubsection{The Referral guidelines}

The Referral Guidelines provide pathways for referrals by LMCs to obstetric and other services. The guidelines acknowledge the role women have in decision-making processes and provide a plan to follow when women decline the care recommended by the guidelines. Consultation with a specialist within 24 hours of PROM at term is recommended, which should involve a three-way conversation that includes the woman, the LMC midwife and the specialist. Care usually remains with the LMC midwife but the specialist should provide care advice $(\mathrm{MOH}, 2012)$. 
Introduction

\subsubsection{DHB Governance}

Each DHB provides, manages and allocates funding for health care services within its district, with the goal of providing effective and equitable health care to its people $(\mathrm{MOH}, 2018 \mathrm{a})$. Care provision policies are written by each DHB to reflect the population, goals and needs, funding, and services available within each district. The NDHB mission "...is to work in partnership under the Treaty of Waitangi with the Northland population to improve population health, reduce inequity and improve the experience of all patients" (NDHB, 2017, p. 11).

\subsubsection{DHB policies}

There is a large pool of published evidence for DHB policy writers to draw from, and variations between DHB policies occur, possibly reflecting the context in which they are written. A stocktake of PROM policies of each New Zealand DHB are collated and are compared in Table 1-3, including the NDHB PROM policy. Referencing for the PROM policies is contained in Appendix 2 as most are not referred to directly in the text; those that are appear in the main Reference list also. In some cases, data were taken from related policies, such as those regarding induction of labour or management of GBS. Some data is absent where the policies provided made no comment.

The duration of recommended maximum expectant management is 96 hours only at NDHB. Five other DHBs allow for varying flexibility over 24 hours, with decision points at 24, 36, 48 or 72 hours. Fourteen DHBs allow a range of 18-24 hours before recommending induction of labour. RANZCOG recommends that this period be up to 24 hours in "... highly selected and well supervised cases" (RANZCOG, 2017, p. 6).

Vaginal prostaglandins are recommended in 15 policies. Vaginal prostaglandin used for women with PROM where the Bishops Score is less than five is supported by RANZCOG (2017). Intravenous oxytocin is recommended as a method of induction by 18 DHB policies, though one policy makes no reference to methods of induction, and one states it is the obstetrician's choice. 
Introduction

IAP is recommended by all DHBs, though the wording around timing varies. Commencing IAP 18 hours after ROM is the most common time, though a few policies state 24 hours, or 18 to 24 hours post-ROM. NDHB recommends IAP for women in active labour following 18 hours or more of ROM. For women who are not labouring, IAP is not recommended until either in active labour or the first digital vaginal examination. The remaining DHBs are divided over whether the IAP should start at 18 or 24 hours from ROM for all women, or if IAP should be delayed until women are either starting an induction process, have had a digital vaginal examination or are in active labour. 
Introduction

Table 1-3 DHB PROM policy comparison

\begin{tabular}{|c|c|c|c|c|c|c|c|c|c|c|}
\hline DHB & Year & $\begin{array}{l}\text { Method of assessing } \\
\text { PROM }\end{array}$ & $\begin{array}{l}\text { Immediate } \\
\text { IOL offered } \\
\text { to low risk } \\
\text { women? }\end{array}$ & $\begin{array}{l}\text { Duration of } \\
\text { expectant mgmt } \\
\text { (hours) }\end{array}$ & Method of IOL & & $\begin{array}{l}\text { Timing of prophylactic } \\
\text { with low GBS risk }\end{array}$ & c antibiotics for women & $\begin{array}{l}\text { First line Prophylactic: } \\
\text { Benzylpenicillin } 1.2 \mathrm{~g} \\
\text { loading dose, then } \\
600 \mathrm{mg} 4 \text { hourly }\end{array}$ & $\begin{array}{l}\text { Period of neonatal } \\
\text { observations }\end{array}$ \\
\hline & & & & & $\mathrm{PGE}_{2} \mathrm{Gel}$ & $\begin{array}{l}\text { Oxytocin } \\
\text { infusion }\end{array}$ & Spontaneous labour & Non-spontaneous labour & & \\
\hline Northland & 2015 & $\begin{array}{l}\text { History, visual, } \\
\text { speculum }\end{array}$ & $\begin{array}{l}\text { Y, the } \\
\text { following } \\
\text { morning }\end{array}$ & 96 & $Y$ for BS 0-5 & $\mathrm{Y}$ & $\begin{array}{l}18 \mathrm{hrs} \text { from ROM, } \\
\text { when in active labour }\end{array}$ & $\begin{array}{l}18 \text { hrs from ROM, when } \\
\text { in active labour/first VE }\end{array}$ & $Y$ & $\begin{array}{l}24 \text { hrs if low risk; } 48 \text { hrs if } \\
\text { high risk }\end{array}$ \\
\hline Waitemata & 2018 & $\begin{array}{l}\text { History, visual, } \\
\text { speculum }\end{array}$ & Y & $18-24$ & $\begin{array}{l}\text { not directly } \\
\text { stated in policy }\end{array}$ & Y & $\begin{array}{l}18 \mathrm{hrs} \text { from ROM, } \\
\text { when labour } \\
\text { established }\end{array}$ & $\begin{array}{l}18 \text { hrs from ROM, when } \\
\text { labour established }\end{array}$ & Y & $\begin{array}{l}24 \text { hrs if low risk; } 48 \text { hrs if } \\
\text { high risk }\end{array}$ \\
\hline Auckland & 2015 & $\begin{array}{l}\text { Visual, speculum, } \\
\text { nitrazine swabs, } \\
\text { amnisure, }\end{array}$ & Y & $18-24$ & $\begin{array}{l}\mathrm{PGE}_{2} \mathrm{Gel} \\
\text { accepted but not } \\
\text { preferred }\end{array}$ & Y & $18 \mathrm{hrs}$ from ROM & $\begin{array}{l}18 \text { hrs from ROM, when } \\
\text { labour established }\end{array}$ & Y & $24-48 \mathrm{hrs}$ \\
\hline $\begin{array}{l}\text { Counties } \\
\text { Manukau }\end{array}$ & 2019 & $\begin{array}{l}\text { History, visual, } \\
\text { speculum, amnisure, } \\
\text { ultrasound }\end{array}$ & $\begin{array}{l}\mathrm{Y} \text {, but EM } \\
\text { preferred }\end{array}$ & 24 & $Y$ for BS 0-4 & Y & $18 \mathrm{hrs}$ from ROM & $\begin{array}{l}18 \text { hrs from ROM when } \\
\text { labour established, or } \\
\text { first VE to give PGE2 gel. }\end{array}$ & Y & $24 \mathrm{hrs}$ if all normal \\
\hline Waikato & 2017 & $\begin{array}{l}\text { History, visual, } \\
\text { speculum }\end{array}$ & $\begin{array}{l}\mathrm{Y} \text {, but EM } \\
\text { preferred }\end{array}$ & $\begin{array}{l}24, \text { deliver in } \\
\text { secondary unit } \\
\text { after } 24 \text { hours } \\
\text { and obstetric } \\
\text { review at } 72 \text { hrs }\end{array}$ & $\mathrm{N}$ & Y & $\begin{array}{l}18 \mathrm{hrs} \text { from ROM, } \\
\text { when in active labour }\end{array}$ & $\begin{array}{l}18 \text { hrs from ROM, when } \\
\text { IOL commenced }\end{array}$ & Y & $\begin{array}{l}48 \text { hrs in hospital or birthing } \\
\text { unit, with FBC and blood } \\
\text { cultures if IAP not given } \\
\text { more than } 4 \text { hrs prior to birth }\end{array}$ \\
\hline Bay of Plenty & 2018 & $\begin{array}{l}\text { History, visual, } \\
\text { speculum, nitrazine } \\
\text { swabs, amnisure }\end{array}$ & Y & 24 & Y & Y & $\begin{array}{l}18 \mathrm{hrs} \text { from ROM, } \\
\text { when in active labour }\end{array}$ & $\begin{array}{l}18 \text { hrs from ROM, when } \\
\text { in active labour }\end{array}$ & Y & $\begin{array}{l}\text { Negotiable for low risk; } 24- \\
48 \text { hrs for high risk. }\end{array}$ \\
\hline Lakes & 2015 & $\begin{array}{l}\text { History, visual, GBS } \\
\text { swab, CTG }\end{array}$ & Y & $24-48$ & Y & Y & $\begin{array}{l}24+\mathrm{hrs} \text {, once labour } \\
\text { est }\end{array}$ & $\begin{array}{l}24+\text { hrs, once IOL } \\
\text { commenced }\end{array}$ & Y & $\begin{array}{l}24 \text { hrs inpatient or at home if } \\
\text { low risk; } 24 \text { hrs inpatient if } \\
\text { high risk. }\end{array}$ \\
\hline Tairawhiti & 2017 & $\begin{array}{l}\text { History, visual, } \\
\text { speculum, amnisure }\end{array}$ & Y & $\begin{array}{l}\text { Until established, } \\
\text { obstetric } \\
\text { assessment if not } \\
\text { est. by 24hrs }\end{array}$ & & Y & $\begin{array}{l}18 \mathrm{hrs} \text { from ROM, } \\
\text { once labour is } \\
\text { established }\end{array}$ & $\begin{array}{l}18 \text { hrs from ROM, once } \\
\text { labour established; at the } \\
\text { start of IOL if there are } \\
\text { risks for GBS colonisation. }\end{array}$ & $\begin{array}{l}\mathrm{Y} ; \text { OR amoxycillin } 2 \mathrm{~g} \\
\text { loading and then } 1 \mathrm{~g} 8 \\
\text { hourly. }\end{array}$ & $\begin{array}{l}24 \mathrm{hrs} \text {, though may do so at } \\
\text { home from } 12 \text { hours if low } \\
\text { risk. }\end{array}$ \\
\hline Hawkes Bay & 2015 & $\begin{array}{l}\text { History, visual, } \\
\text { speculum, CTG, } \\
\text { nitrazine swabs, } \\
\text { ferning on } \\
\text { microscopy, USS. }\end{array}$ & $\begin{array}{l}\text { Y from 8am } \\
\text { mane, or } \\
\text { later that } \\
\text { day if SROM } \\
\text { early }\end{array}$ & $\begin{array}{l}24 \mathrm{hrs}+\text { to } 8 \mathrm{am} \\
\text { the next day }\end{array}$ & Obstetric choice & $\begin{array}{l}\text { Obstetric } \\
\text { choice }\end{array}$ & $\begin{array}{l}18 \mathrm{hrs} \text { from ROM, } \\
\text { once labour is } \\
\text { established }\end{array}$ & $\begin{array}{l}18 \mathrm{hrs} \text { from ROM, once } \\
\text { labour is established }\end{array}$ & Y & $24 \mathrm{hrs}$, location not stated. \\
\hline
\end{tabular}




\begin{tabular}{|c|c|c|c|c|c|c|c|c|c|c|}
\hline$\overline{\mathrm{DHB}}$ & Year & $\begin{array}{l}\text { Method of assessing } \\
\text { PROM }\end{array}$ & $\begin{array}{l}\text { Immediate } \\
\text { IOL offered } \\
\text { to low risk } \\
\text { women? }\end{array}$ & $\begin{array}{l}\text { Duration of } \\
\text { expectant mgmt } \\
\text { (hours) }\end{array}$ & Method of IOL & & $\begin{array}{l}\text { Timing of prophylacti } \\
\text { with low GBS risk }\end{array}$ & c antibiotics for women & $\begin{array}{l}\text { First line Prophylactic: } \\
\text { Benzylpenicillin } 1.2 \mathrm{~g} \\
\text { loading dose, then } \\
600 \mathrm{mg} 4 \text { hourly }\end{array}$ & $\begin{array}{l}\text { Period of neonatal } \\
\text { observations }\end{array}$ \\
\hline & & & & & $\mathrm{PGE}_{2}$ Gel & $\begin{array}{l}\text { Oxytocin } \\
\text { infusion }\end{array}$ & Spontaneous labour & Non-spontaneous labour & & \\
\hline Taranaki & 2018 & $\begin{array}{l}\text { History, visual, } \\
\text { speculum, Amnisure }\end{array}$ & $\mathrm{N}$ & $18 \mathrm{hrs}$ & Y dinoprostone & $\mathrm{Y}$ & $\begin{array}{l}18 \mathrm{hrs} \text { from ROM, in } \\
\text { established labour }\end{array}$ & $\begin{array}{l}18 \text { hrs from ROM with } \\
\text { start of IOL/ first VE if } \\
\text { earlier. }\end{array}$ & $Y$ & $\begin{array}{l}24 \mathrm{hrs} \text { only if IAP }<4 \mathrm{hrs} \text { or } \\
\text { not given. }\end{array}$ \\
\hline Whanganui & 2016 & $\begin{array}{l}\text { Visual, speculum, } \\
\text { Amnisure, USS, CTG }\end{array}$ & $\begin{array}{l}\text { Y, but EM } \\
\text { preferred }\end{array}$ & $72 \mathrm{hrs}$ & $\begin{array}{l}\text { not directly } \\
\text { stated }\end{array}$ & $\mathrm{Y}$ & \multicolumn{2}{|c|}{$\begin{array}{l}\text { If low risk, oral erythromycin from } 18 \text { hrs post } \\
\text { ROM, then IV benzylpenicillin when established } \\
\text { in labour; if high risk oral erythromycin } \\
\text { immediately from ROM until established, then IV } \\
\text { benzylpenicillin. }\end{array}$} & Y & $\begin{array}{l}24 \text { hrs inpatient or at home if } \\
\text { low risk; } 24 \text { hrs inpatient if } \\
\text { high risk. }\end{array}$ \\
\hline Mid Central & 2016 & $\begin{array}{l}\text { History, visual, } \\
\text { speculum, amnistix, } \\
\text { USS }\end{array}$ & $\mathrm{N}$ & $18 \mathrm{hrs}$ & not stated & not stated & $18 \mathrm{hrs}$ from ROM & $\begin{array}{l}18 \text { hrs from ROM or from } \\
\text { the first VE; oral } \\
\text { Erythromycin may be } \\
\text { offered prior to labour } \\
\text { establishing. }\end{array}$ & Y & $\begin{array}{l}48 \mathrm{hrs} \text { if IAP given, though } \\
\text { can be reduced to } 24 \mathrm{hrs} \text { if } \\
\text { otherwise low risk. }\end{array}$ \\
\hline Wairarapa & 2015 & $\begin{array}{l}\text { History, visual, } \\
\text { speculum, USS, VE if } \\
\text { cervix not } \\
\text { visualised/query } \\
\text { presenting part }\end{array}$ & $\mathrm{N}$ & $24-36 \mathrm{hrs}$ & $Y$ & Y & $18-24$ hrs from ROM & 18-24 hrs from ROM & $\begin{array}{l}\text { Benzylpenicillin } 2.4 \mathrm{~g} \\
\text { loading dose, then } \\
1.2 \mathrm{~g} 4 \text { hourly. }\end{array}$ & 24 hrs inpatient \\
\hline Hutt Valley & 2013 & $\begin{array}{l}\text { History, visual, } \\
\text { speculum }\end{array}$ & $\mathrm{Y}$ & $18 \mathrm{hrs}$ & & & $18 \mathrm{hrs}$ from ROM & $\begin{array}{l}18 \text { hrs from ROM with } \\
\text { start of IOL }\end{array}$ & $\mathrm{Y}$ & $\begin{array}{l}24 \mathrm{hrs} \text {, recommended to be } \\
\text { in a facility }\end{array}$ \\
\hline $\begin{array}{l}\text { Capital and } \\
\text { Coast }\end{array}$ & 2015 & $\begin{array}{l}\text { History, visual, } \\
\text { speculum, CTG }\end{array}$ & $Y$, preferred & $\begin{array}{l}24 \mathrm{hrs} \text {, preferably } \\
\text { by } 1400 \mathrm{hrs}\end{array}$ & & Y & $\begin{array}{l}18 \mathrm{hrs} \text {, in established } \\
\text { labour }\end{array}$ & $\begin{array}{l}18 \mathrm{hrs}, \text { when in } \\
\text { established labour. }\end{array}$ & $\mathrm{Y}$ & $\begin{array}{l}24 \mathrm{hrs} \text {, but can be at home if } \\
\text { low risk }\end{array}$ \\
\hline $\begin{array}{l}\text { Nelson- } \\
\text { Marlborough }\end{array}$ & 2014 & $\begin{array}{l}\text { History, visual, } \\
\text { speculum, CTG }\end{array}$ & $\mathrm{Y}$ & $24 \mathrm{hrs}$ & Y & Y & $\begin{array}{l}18 \text { hrs from ROM, in } \\
\text { established labour }\end{array}$ & $\begin{array}{l}\text { Once labour established; } \\
\text { or at the start of IOL if } \\
\text { there are risks for GBS } \\
\text { colonisation }\end{array}$ & Y & $\begin{array}{l}24-48 \text { hrs in a facility } \\
\text { depending on risk factors, } \\
\text { but may go home after } 24 \\
\text { hrs if parents understand the } \\
\text { situation }\end{array}$ \\
\hline West Coast & 2017 & $\begin{array}{l}\text { History, visual, } \\
\text { speculum, nitrazine } \\
\text { swabs }\end{array}$ & $\mathrm{N}$ & $24 \mathrm{hrs}$ & $\begin{array}{l}\text { Y dinoprostone } \\
\text { only }\end{array}$ & Y & $24 \mathrm{hrs}$ from ROM & $\begin{array}{l}24 \mathrm{hrs} \text { from ROM, at start } \\
\text { of } \mathrm{IOL} \text { process }\end{array}$ & Y & $\begin{array}{l}24 \text { hrs if less than } 2 \text { doses of } \\
\text { IAP has been given }\end{array}$ \\
\hline Canterbury & 2017 & $\begin{array}{l}\text { History, visual, } \\
\text { speculum, nitrazine } \\
\text { swabs }\end{array}$ & $\mathrm{N}$ & $24 \mathrm{hrs}$ & $\begin{array}{l}\text { Y dinoprostone } \\
\text { only }\end{array}$ & Y & $24 \mathrm{hrs}$ from ROM & $\begin{array}{l}24 \mathrm{hrs} \text { from ROM, at start } \\
\text { of } \mathrm{OL} \text { process }\end{array}$ & Y & $\begin{array}{l}24 \text { hrs if less than } 2 \text { doses of } \\
\text { IAP has been given }\end{array}$ \\
\hline $\begin{array}{l}\text { South } \\
\text { Canterbury }\end{array}$ & 2014 & $\begin{array}{l}\text { History, visual, } \\
\text { speculum, } \\
\text { nitrazine/amnistix }\end{array}$ & N & $18 \mathrm{hrs}$ inpatient & $\mathrm{Y}$ & Y & $\begin{array}{l}18 \mathrm{hrs} \text { from ROM, in } \\
\text { established labour }\end{array}$ & $\begin{array}{l}18 \mathrm{hrs} \text { from ROM (oral if } \\
\text { not in labour) }\end{array}$ & $\begin{array}{l}\text { Amoxycillin } 2 \mathrm{~g} \text { loading } \\
\text { dose then } 1 \mathrm{~g} 4 \text { hourly. }\end{array}$ & $\begin{array}{l}24 \text { - } 48 \text { hrs, may be at home } \\
\text { if low risk for GBS or mother } \\
\text { received IAP } 4 \text { hrs + prior to } \\
\text { birth. }\end{array}$ \\
\hline Southern & 2015 & $\begin{array}{l}\text { History, visual, } \\
\text { speculum, amnisure, } \\
\text { ultrasound }\end{array}$ & $\mathrm{N}$ & $18 \mathrm{hrs}$ & $\mathrm{Y}$ & Y & $\begin{array}{l}18 \mathrm{hrs} \text { from ROM, in } \\
\text { established labour }\end{array}$ & $\begin{array}{l}18 \mathrm{hrs} \text { from ROM, when } \\
\text { in established labour or } \\
\text { at start of } \mathrm{IOL}\end{array}$ & $Y$ & $\begin{array}{l}48 \mathrm{hrs} \text {, though if IAP given }>4 \\
\text { hrs prior to birth, may return } \\
\text { home after } 24 \mathrm{hrs} \text { if parents } \\
\text { understand the situation }\end{array}$ \\
\hline
\end{tabular}


Introduction

\subsection{Summary}

This chapter has introduced the topic of the management of PROM. It has outlined the demography of the NDHB area and local maternity services provided. It has explored the identification and risks of PROM, and of GBS. The medico-legal context is described, including detailing the practice guidelines, DHB governance and DHB policy variations.

\subsection{Research aim, questions, and objectives}

The aim of this study was to evaluate and describe the processes of care, and outcomes for women birthing at NDHB facilities following PROM. The research questioned whether the NDHB PROM policy was applied in practice, and if that policy safely met the needs of birthing women experiencing PROM. The objectives of this study were to: obtain adequate sample numbers; use appropriate statistical analysis to identify any issues for women birthing with PROM, and to present recommendations for practice, education, and research.

\subsection{Overview of the thesis}

This thesis is comprised of five chapters. This introduction and background have outlined the topic of PROM and the setting in which the NDHB PROM policy is applied. The next chapter will describe a literature review of original research publications, most which have been published in the past decade. Chapter three will outline the methodology and methods used in conducting the data collection and analysis for this project. The findings of the data analysis are presented in detail in chapter four, and the thesis is concluded in chapter 5 with in-depth discussion of the findings, with recommendations for future developments in practice, education, and research. 


\section{$2 \quad$ Literature Review}

The aim of this research project was to evaluate the utilisation of the NDHB PROM policy and whether it safely met the needs of the women (and their babies) birthing at NDHB facilities. PROM at term presents several decisions to be made by women and their midwives or obstetricians, including whether expectant management is appropriate, timing and mode of any induction procedures, and to consider the need for IAP. In preparation for data collection, a literature review was conducted to examine recent topical research and to identify key factors to include in data collection for this study. In this chapter relevant publications have been grouped based on topic and are explored to identify and discuss findings from recent research on PROM.

\section{$2.1 \quad$ Keywords}

Artificial rupture of membranes, Group $\beta$ Streptococcus, prelabour rupture of membranes at term ( $\geq 37$ weeks), spontaneous rupture of membranes, induction of labour.

\subsection{The aim and objectives of the literature review}

This review aims to examine evidence related to the management of PROM, to:

- contribute to the body of clinical knowledge available,

- provide a critical perspective of the existing literature,

- create a foundation of evidence for practice,

- identify any gaps in research.

\subsection{Search strategy}

The search and review were undertaken in several stages, and is detailed in the PRISMA diagram, see Figure 2-1. CINAHL, the Cochrane Database of Systematic Reviews, Medline, Pubmed, Science Direct, Scopus, Springer Link and Google were searched in May 2019 and again in March 2020 for articles published between 2008 and 2020. 
The search terms used were "rupture of membranes", "prelabour" and "premature", paired with "term". The word "prolonged" generally redirected the search to "premature" and did not discover useful material. These terms led to the discovery of 473 articles, with a further 24 articles located through basic Google searching or following citation links. Screening for basic relevance and duplication reduced the number to 326 . Inclusion and exclusion criteria were applied as below and reduced the number of publications included to 117 .

\subsubsection{Inclusion criteria:}

- Randomised controlled trials (RCT), prospective or retrospective observational or cohort studies.

\subsubsection{Exclusion criteria:}

- Not published in English.

- Full article not available.

- Not related or focusing on PROM at term.

\subsection{Selection of studies}

Critical analysis of the content and relevance of the literature was applied. Literature was managed in Endnote and an Excel matrix summarised publications and provided a comparison mechanism (Garrard, 2011). This process eliminated publications with insufficient relevance, editorials or opinion pieces, articles published prior to 2008 , and those with results indistinguishable from preterm results. Conversely, three older publications were reintroduced when identified as seminal or uniquely positioned in the field. Grey literature and meta-analysis publications were excluded in favour of a focus on only primary publications.

The final number of publications included in this literature review was 35 , from a variety of international sources. In many studies, numbers were too small to provide statistically significant findings, and often management differed significantly from that used in New Zealand, limiting the generalisability to New Zealand maternity care. 


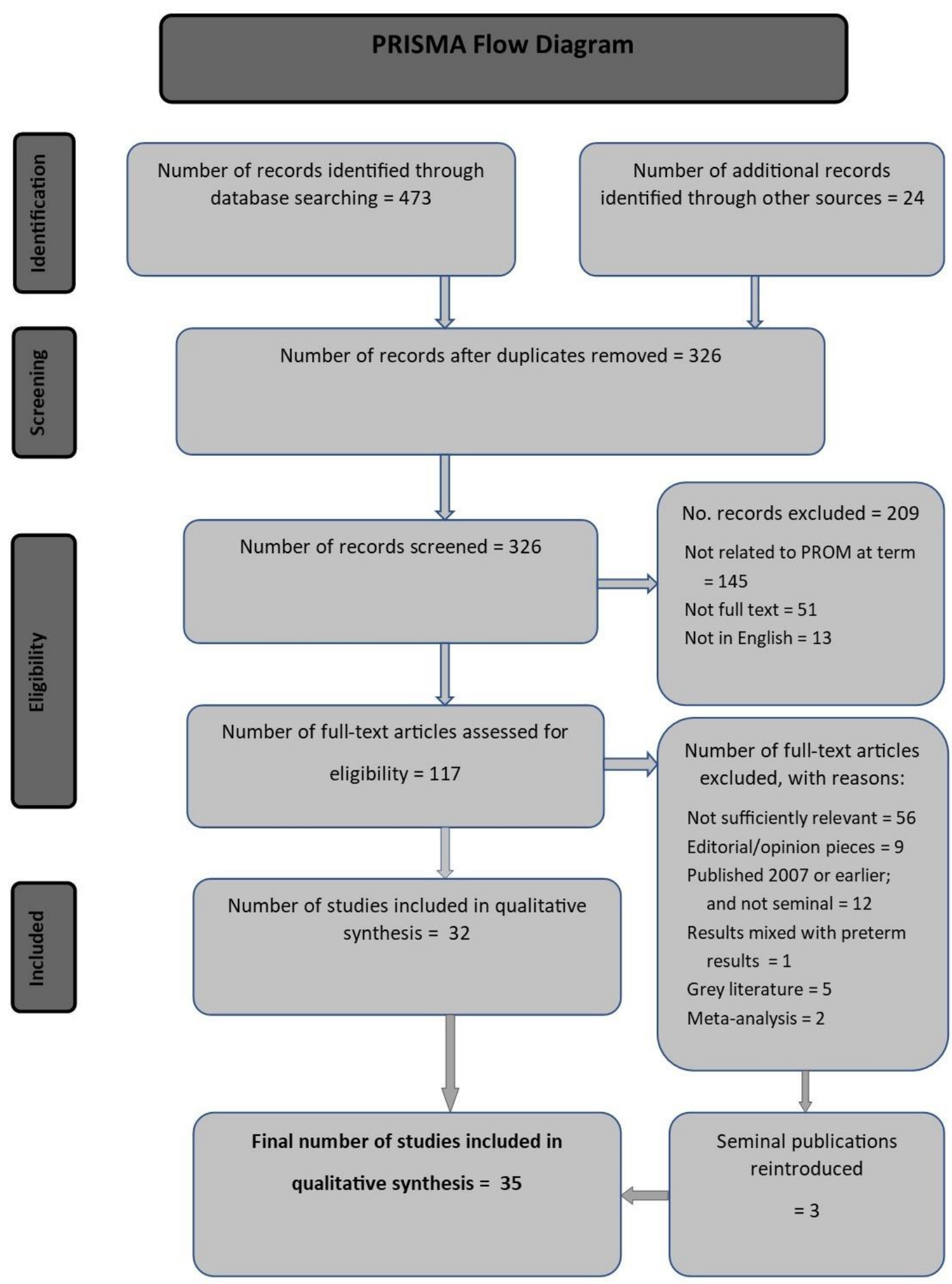

Figure 2-1 PRISMA Flow Diagram for Literature Review

Adapted from: Liberati et al. (2009). 


\subsection{Data extraction}

The Excel matrix was used to record study methods, numbers of participants, quality of randomisation and any blinding, primary and secondary outcomes, findings, and my own comments about each publication. Each publication was allocated to one of four central themes:

- Outcomes after PROM

- Management of PROM

- Induction methods for women with PROM

- $\quad$ IAP after PROM

Some publications could have been allocated into more than one category; however, this was avoided for simplicity.

\subsection{Data synthesis}

Each group of publications has been compared to identify key findings, which are presented in table form, though reporting styles varied between studies. Reporting is summarised as a narrative synthesis due to different reporting styles within the studies, and because approaches are variable enough that a meta-analysis is not easily produced.

\subsection{Findings}

\subsubsection{Outcomes following PROM}

Maternal and neonatal birth outcomes following PROM were the subject of six retrospective observational studies included in this section. Results reported by publications in this group were largely given as percentages. Key findings included duration of labour, mode of birth, maternal infections, postpartum haemorrhage and neonatal morbidity, and are shown in Table 2-1. 
Literature Review

Table 2-1 Outcomes following PROM

\begin{tabular}{|c|c|c|c|c|c|c|c|c|c|c|}
\hline Author & Year & $\mathrm{n}=$ & Study arms & $\begin{array}{l}\text { Duration of } \\
\text { labour }\end{array}$ & $\begin{array}{l}\text { Vaginal } \\
\text { birth }\end{array}$ & $\begin{array}{l}\text { Instrumental } \\
\text { birth }\end{array}$ & $\begin{array}{l}\text { Caesarean } \\
\text { birth }\end{array}$ & Chorioamnionitis & PPH & Neonatal outcomes \\
\hline \multirow[t]{3}{*}{ Li, Wang, Li \& Yang } & 2011 & 579 & $\begin{array}{l}\text { (1) spontaneous labour within } 12 \\
\text { hours of PROM }\end{array}$ & $\begin{array}{l}13.1+/-5.4 \mathrm{hrs} \\
(p<0.05)\end{array}$ & $69.90 \%$ & $10.96 \%$ & $19.18 \%$ & $3.40 \%$ & & $13.7 \%$ neonatal infection \\
\hline & & & $\begin{array}{l}\text { (2) IOL with oxytocin after } 12 \text { hours } \\
\text { of PROM }\end{array}$ & $\begin{array}{l}26.9+/-11.1 \mathrm{hrs} \\
(p<0.05)\end{array}$ & $54.70 \%$ & $8.97 \%$ & $45.73 \%$ & $10.70 \%$ & & $21.8 \%$ neonatal infection \\
\hline & & & (3) LSCS immediately after PROM & $\begin{array}{l}4.2+/-2.4 \text { hrs } \\
(p<0.05)\end{array}$ & $0 \%$ & $0 \%$ & $100 \%$ & $1.90 \%$ & & $3.8 \%$ neonatal infection \\
\hline $\begin{array}{l}\text { Mynarek, Maren; } \\
\text { Bjellmo, Solveig; } \\
\text { Lydersen, Stian; Strand, } \\
\text { Kristin Melheim; Afset, } \\
\text { Jan Egil; Andersen, Guro } \\
\text { L; Vik, Torstein }\end{array}$ & 2020 & 65091 & $\begin{array}{l}\text { Babies born following PROM; either } \\
\text { born after } 12 \text { - } 24 \text { hours } \\
\text { (intermediate, } n=34759 \text { ) or born } \\
\text { after } 24 \text { hours (prolonged, } n=30332 \text { ) }\end{array}$ & & & & & & & $\begin{array}{l}\text { Cerebral palsy. OR for intermediate } \\
\text { group }(1.16,95 \% \mathrm{Cl},[0.83,1.61]) . \mathrm{OR} \\
\text { for prolonged group }(1.61,95 \% \mathrm{Cl} \text {, } \\
[1.19,2.18], p=0.002)\end{array}$ \\
\hline \multirow{3}{*}{$\begin{array}{l}\text { Namli Kalem, Köşüş, } \\
\text { Kamalak, Köşüş \& Kalem }\end{array}$} & 2017 & 82 & (1) latent labour duration $>6$ hours & & $3.50 \%$ & & $4.00 \%$ & & & \\
\hline & & & (2) latent labour duration 6-12 hours & & $61.40 \%$ & & $24.00 \%$ & & & \\
\hline & & & (3) latent labour duration $>12$ hours & & $35.10 \%$ & & $72.00 \%$ & & & \\
\hline $\begin{array}{l}\text { Seaward, Hannah, } \\
\text { Myhr, Farine, Ohlsson, } \\
\text { Wang, ... \& Ohel }\end{array}$ & 1998 & $\begin{array}{l}5028 \\
\text { babies }\end{array}$ & Secondary multivariate analysis & & & & & $7 \%$ & & $2.6 \%$ neonatal infection \\
\hline $\begin{array}{l}\text { Tran, Cheng, Kaimal \& } \\
\text { Caughey }\end{array}$ & 2008 & 3841 & $\begin{array}{l}\text { Duration of labour in } 2 \text { hourly } \\
\text { intervals }\end{array}$ & & & & & $\begin{array}{l}\text { At } 18 \text { hours: } 4.0 \% \\
\text { before vs } 13.4 \% \\
\text { after, } p<0.05 \text {. } \\
\text { Chorio significant } \\
\text { from } \geq 12 \text { hours (OR } \\
2.3,95 \% \mathrm{Cl},[1.2, \\
4.4] \text { ). }\end{array}$ & $\begin{array}{l}\text { At } 18 \text { hours: } 14.5 \% \text { before } \\
\text { and } 24.0 \% \text { after } p<0.05 \text {. } \\
\text { Significant from } 8 \text { hours } \\
\text { (OR } 2.8,95 \% \mathrm{Cl},[1.1-7.2] \text { ) } \\
\text { with risk decreasing over } \\
\text { time but remaining } \\
\text { significant. At } 18 \text { hours the } \\
\text { split is OR } 1.3,95 \% \mathrm{Cl}[1.0- \\
1.6]) \text {. }\end{array}$ & \\
\hline Xia, Li, Li, Liang \& Xu & 2015 & 13927 & $\begin{array}{l}\text { PROM vs membranes intact prior to } \\
\text { labour }\end{array}$ & & & & $\begin{array}{l}55.1 \% \text { vs } \\
42.5 \% \\
p=0.00\end{array}$ & & $5.1 \%$ vs $3.7 \%, p<0.01$ & Asphyxia $3.2 \%$ vs $2.2 \% p=0.00$ \\
\hline
\end{tabular}


Duration of labour reported by Li, Wang, Li, and Yang (2011) was unsurprisingly longer for women who did not spontaneously establish into labour, but had induction of labour with oxytocin after 12 hours expectant management. This topic will be returned to later in this chapter in comparisons of expectant and active management protocols.

Mode of birth was reported by Namli Kalem, Köşüş, Kamalak, Köşüş, and Kalem (2017), who proposed that PROM lasting over 12 hours without labour establishing spontaneously implies a pathological cause. They reported an increasing incidence of caesarean birth as latent labour duration increased. Where cervical dilatation was less than $3 \mathrm{~cm}$ at admission, the rate of caesarean birth was $43.1 \%$, versus $9.7 \%$ for women with dilatation $\geq 3 \mathrm{~cm}$. The rate of caesarean birth was also higher for women following PROM than for women in spontaneous labour with intact membranes for Xia, Li, Li, Liang, and Xu (2015). Li et al. (2011) linked oxytocin to induce labour after 12 hours of expectant management to an increased number of caesarean births.

Chorioamnionitis ranged in incidence from $1.9 \%$ for caesarean birth immediately after PROM, to $13.4 \%$ for women birthing after 18 hours post-ROM. Durations of PROM of over 12 hours was significantly associated with increased risk of chorioamnionitis (Tran, Cheng, Kaimal, \& Caughey, 2008), and endometritis was significantly associated with duration of PROM of over 16 hours (OR 2.5, 95\% Cl, [1.1-5.6]).

Postpartum haemorrhage was linked to PROM by two studies. Xia et al. (2015) found an incidence of $5.1 \%$ versus $3.7 \%$ for women birthing without PROM, $(p<0.01)$. Tran et al. (2008) reported an incidence of $14.5 \%$ for women birthing before 18 hours had elapsed, versus $24 \%$ for women birthing after 18 hours from ROM, $(p<0.05)$. The incidence of postpartum haemorrhage was significant from eight hours onwards (OR 2.8, 95\% Cl, [1.17.2]), with risk decreasing over time but remaining significant.

Neonatal outcomes included infection, cerebral palsy and asphyxia. Seaward et al. (1998) provided a secondary analysis of the Hannah et al. (1996) study, that included 5028 neonates and reported an overall incidence of neonatal infection of $2.6 \%$. Predictive factors for neonatal infection were identified as: maternal clinical chorioamnionitis (OR 5.89, $p<0.0001$ ); positive GBS status (OR 3.08, $p<0.0001$ ); seven to eight vaginal examinations (compared to 0 to 2 ), (OR 2.37, $p=0.04$ ); and increasing duration from ROM to active labour 
when compared to 12 hours or less following PROM: 24-47 hours to active labour (OR 1.97, $p=0.02$ ); and $>48$ hours (OR 2.25, $p=0.01$ ).

Neonatal infection rates varied considerably in the analysis by Li et al. (2011). The immediate caesarean group had an incidence of 3.8\% neonatal infection, but concerningly the babies born following both spontaneous (13.7\%) and induced labour (21.8\%) developed infections. This was attributed to an increased number of vaginal examinations received during longer labours.

Cerebral palsy was linked to duration of PROM by Mynarek et al. (2020), who found increased risk of cerebral palsy even at a duration of 12-24 hours from ROM, with the risk higher at $\geq 24$ hours duration, though overall risk remained low. Asphyxia (not defined by the authors) and hypoxic-ischaemic injuries were also reported at higher incidence following PROM compared to babies born without PROM.

\subsubsection{Management of PROM}

Active versus expectant management of PROM was the central focus of ten publications, including six randomised controlled trials, one prospective comparison and three retrospective studies (Table 2-2). The Hannah, et al., (1996) TERMPROM study was included as seminal research which has not been superseded by any other study to the same scale or quality (Middleton et al., 2017). The TERMPROM study included 5041 women with PROM, with four study arms comprising active or expectant management for up to 96 hours, with any induction commenced with either vaginal prostaglandin gel or intravenous oxytocin. The primary outcome was neonatal infection, with secondary outcomes of caesarean birth and other maternal or neonatal health indicators. 


\section{Literature Review}

\section{Table 2-2 Management of PROM}

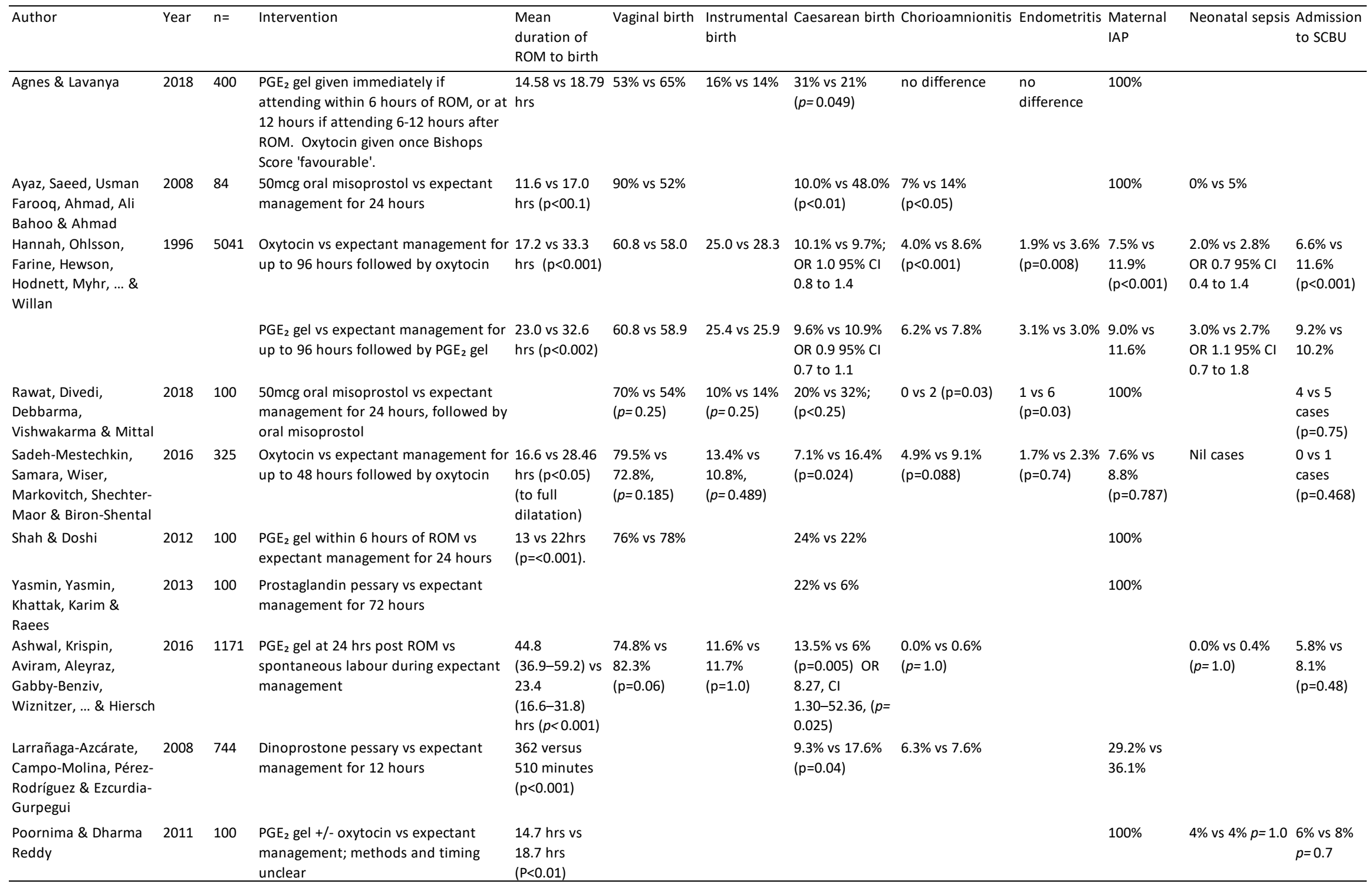


Duration of expectant management, timing, and methods of induction of labour varied between studies. Outcomes reported by studies in this group included duration of labour, caesarean birth, chorioamnionitis, endometritis, maternal IAP or antibiotic treatment, neonatal sepsis, and neonatal admission to neonatal care unit. Reporting by studies in this group was done with a mixture of percentages and odds-ratios.

Active management of labour was commenced either immediately or within the first six hours of PROM. The duration of expectant management varied significantly and included: 12 hours (Agnes \& Lavanya, 2018; Larrañaga-Azcárate, Campo-Molina, Pérez-Rodríguez, \& Ezcurdia-Gurpegui, 2008); 24 hours (Ayaz et al., 2008; Rawat, Divedi, Debbarma, Vishwakarma, \& Mittal, 2018; Shah \& Doshi, 2012); 48 hours (Sadeh-Mestechkin et al., 2016); 72 hours (Yasmin, Yasmin, Khattak, Karim, \& Raees, 2013); and 96 hours (Hannah et al., 1996). It was difficult to discern the management protocols in the publication by Poornima and Dharma Reddy (2011) due to poor quality of writing. In all studies women were admitted to hospital for the duration of their expectant management, except in Hannah et al., where approximately $40 \%$ of women returned to their homes.

Duration from ROM to birth was reported by nine publications, though one gave durations only in a distribution table (Rawat et al., 2018), making comparison difficult. The remaining eight studies all reported duration from ROM to birth or full dilation to be reduced using active management of labour, with seven providing significant $p$ values (Agnes \& Lavanya, 2018; Ashwal et al., 2016; Ayaz et al., 2008; Hannah et al., 1996; Larrañaga-Azcárate et al., 2008; Poornima \& Dharma Reddy, 2011; Sadeh-Mestechkin et al., 2016; Shah \& Doshi, 2012). Women in the Hannah et al. (1996) trial who were actively induced with oxytocin experienced the fewest digital vaginal examinations, shortest duration to active labour and shortest duration of active labour, compared to the other management protocols.

Mode of birth was reported in seven studies, although Yasmin et al. (2013) only reported on caesarean births, and Poornima and Dharma Reddy (2011) claimed results but did not provide figures. Vaginal birth was more frequent for women with induced labour versus expectant management in four studies (Ayaz et al., 2008; Hannah et al., 1996; Rawat et al., 2018; Sadeh-Mestechkin et al., 2016), and more frequent for women with expectant management in three studies (Agnes \& Lavanya, 2018; Ashwal et al., 2016; Shah \& Doshi, 
2012). Instrumental births were reported by four studies and also showed conflicting results with no significant $p$ values (Agnes \& Lavanya, 2018; Poornima \& Dharma Reddy, 2011; Rawat et al., 2018; Sadeh-Mestechkin et al., 2016). Similarly, caesarean births were also reported with enough variation in results that no conclusion could be drawn.

Chorioamnionitis was reported as occurring more frequently in women using expectant management in six studies (Ashwal et al., 2016; Ayaz et al., 2008; Hannah et al., 1996; Larrañaga-Azcárate et al., 2008; Rawat et al., 2018; Sadeh-Mestechkin et al., 2016), with a fifth stating no difference between management methods (Agnes \& Lavanya, 2018). Endometritis was also more common in women using expectant management in three studies (Hannah et al., 1996; Rawat et al., 2018; Sadeh-Mestechkin et al., 2016), and women in the immediate induction with oxytocin group had the lowest incidence of endometritis in the Hannah et al. (1996) study.

IAP was given to all participants in six studies (Agnes \& Lavanya, 2018; Ayaz et al., 2008; Poornima \& Dharma Reddy, 2011; Rawat et al., 2018; Shah \& Doshi, 2012; Yasmin et al., 2013). Three of those studies provided neonatal sepsis data, although two had nonsignificant results (Ashwal et al., 2016; Poornima \& Dharma Reddy, 2011). Yasmin et al. (2013) reported 5\% neonatal sepsis rates for babies born to women using expectant management for up to 24 hours. No statistically significant differences were found between study arms by Hannah et al. (1996), though the active management with oxytocin study arm had less neonatal antibiotics and neonatal admissions, and four babies in the expectant management group died (two from asphyxia, one from GBS infection and one from birth trauma).

The comparison of outcomes in the smaller studies comparing women with active management of PROM versus expectant management does not provide consistent findings that can be used as evidence for practice. The Hannah et al. (1996) study did achieve statistical significance and despite finding immediate induction of labour using oxytocin to have slightly better outcomes, it also endorsed the expectant management protocol that is applied by NDHB (Bailey, 2015). 


\subsubsection{Methods of induction of labour following PROM}

Comparisons of methods of induction of labour following PROM were the focus of much of recent research related to PROM (Table 2-3). All twelve clinical trials in this group compared the use of one medication or route of administration to another. The most common medications were vaginal prostaglandins - generally prostaglandin $\mathrm{E}_{2}\left(\mathrm{PGE}_{2}\right)$ gel, but also dinoprostone pessaries; misoprostol tablets (either vaginal, oral, or sublingual administration); and IV oxytocin. One study reviewed the use of cervical balloons ${ }^{9}$ and one study looked at the use of laminaria tents ${ }^{10}$. All studies used IV oxytocin as a subsequent step to any cervical ripening method if required to induce or augment labour.

This grouping of studies included nine randomised comparative trials, one prospective cohort study, and two retrospective studies. Two of the randomised studies stated they had control groups, however the standard care described by each could be the intervention. Key outcomes were similar to previously discussed studies, and included mean duration of ROM to birth, vaginal, instrumental and caesarean birth, chorioamnionitis and SCBU admission; however, variation of induction methods and timing between the studies makes it impossible to state any consistent findings for any key outcomes. This part of the literature review will therefore provide a narrative comparison of the various induction methods.

\footnotetext{
${ }^{9}$ Fluid filled catheter balloon placed either above or on either side of the cervix, which creates mechanical dilation due to pressure, tension and weight (Cabrera et al., 2016).

${ }^{10}$ Hygroscopic rods bundled and placed in the cervix, which create mechanical dilation as the rods absorb moisture (Kurasawa et al., 2014).
} 
Table 2-3 Induction of labour following PROM

\begin{tabular}{|c|c|c|c|c|c|c|c|c|c|}
\hline Author & Year & $n=$ & Method & $\begin{array}{l}\text { Mean duration ROM } \\
\text { to birth }\end{array}$ & Vaginal birth & Instrumental birth & Caesarean birth & Chorioamnionitis & SCBU admission \\
\hline $\begin{array}{l}\text { Cabrera, Quiñones, Durie, } \\
\text { Rust, Smulian \& Scorza }\end{array}$ & 2016 & 124 & $\begin{array}{l}\text { Balloon }+/ \text { - oxytocin } \\
\text { vs oxytocin alone }\end{array}$ & $\begin{array}{l}21.5 \mathrm{hrs} \text { vs } 18.4 \mathrm{hrs} \\
(\mathrm{p}=0.03) \mathrm{RR} 1.0195 \% \\
\mathrm{Cl}(0.99-1.04)\end{array}$ & & & $\begin{array}{l}45.2 \% \text { vs } 22.0 \% \\
(p=0.007)\end{array}$ & $\begin{array}{l}28.6 \% \text { vs } 15.9 \% \\
(p=0.10)\end{array}$ & $\begin{array}{l}21.4 \% \text { vs } 8.5 \% \\
(p=0.07)\end{array}$ \\
\hline $\begin{array}{l}\text { Güngördük, Asicioglu, } \\
\text { Besimoglu, Güngördük, } \\
\text { Yildirm, Ark \& Sahbaz }\end{array}$ & 2012 & 450 & $\begin{array}{l}\text { Dinoprostone pessary } \\
+ \text { +- oxytocin vs } \\
\text { oxytocin only }\end{array}$ & & $\begin{array}{l}78.5 \% \text { vs } 63.3 \% \mathrm{RR} \\
1.23 ; 95 \% \mathrm{Cl}(1.09- \\
1.39) ;(p=0.001)\end{array}$ & 7 vs 5 cases & $30.3 \%$ vs $18.4 \%$ & $\begin{array}{l}1.4 \% \text { vs } 3.1 \% \\
(p=0.33) \text { RR } 2.31 \\
95 \% \text { Cl }(0.60-8.82)\end{array}$ & $\begin{array}{l}2.7 \% \text { vs } 1.8 \% \\
(p=0.54) \operatorname{RR} 0.66 \\
95 \% \mathrm{Cl}(0.18-2.30)\end{array}$ \\
\hline $\begin{array}{l}\text { Kurasawa, Yamamoto, } \\
\text { Usami, Mochimaru, } \\
\text { Mochizuki, Aoki, ... \& } \\
\text { Hirahara }\end{array}$ & 2014 & 782 & $\begin{array}{l}\text { Laminaria tent vs } \\
\text { standard care }\end{array}$ & $\begin{array}{l}19.6 \mathrm{hrs} \text { vs } 17.1 \mathrm{hrs} \\
(p<0.18)\end{array}$ & & & $6.5 \%$ vs $5.1 \%(p<0.6)$ & & \\
\hline $\begin{array}{l}\text { Bricker, Peden, Tomlinson, } \\
\text { Al-Hussaini, Idama, } \\
\text { Candelier, et al. }\end{array}$ & 2008 & 758 & $\begin{array}{l}\text { Oral misoprostol vs } \\
\text { dinoprostone pessary } \\
\text { +/- oxytocin }\end{array}$ & & $\begin{array}{l}\text { (within } 24 \text { hours) } \\
24 \% \text { vs } 31 \%, R R \\
0.79,95 \% \mathrm{Cl}(0.63- \\
1.00)\end{array}$ & $\begin{array}{l}16 \% \text { vs } 15 \% \text { RR } 1.02 ; \\
95 \% \text { Cl (0.73 - 1.42) }\end{array}$ & $\begin{array}{l}14 \% \text { vs } 18 \%, \mathrm{RR} \\
0.79 ; 95 \% \mathrm{Cl}(0.57- \\
1.09)\end{array}$ & $\begin{array}{l}2 \% \text { vs } 2 \% \text { RR 1.36; } \\
95 \% \mathrm{Cl}(0.48-3.89)\end{array}$ & $\begin{array}{l}2 \% \text { vs } 3 \% \text { RR } 0.61 \\
95 \% \mathrm{Cl}(0.22-1.67)\end{array}$ \\
\hline $\begin{array}{l}\text { Mozurkewich, Horrocks, } \\
\text { Daley, Von Oeyen, } \\
\text { Halvorson, Johnson, et al }\end{array}$ & 2003 & 305 & $\begin{array}{l}\text { Oral misoprostol vs } \\
\text { oxytocin }\end{array}$ & 11.9 hrs vs 11.8 hrs & & $\begin{array}{l}10.7 \% \text { vs } 12.4 \% \\
\text { ( } p=\text { not significant) }\end{array}$ & $\begin{array}{l}20.1 \% \text { vs } 19.9 \% \\
\text { ( } p=\text { not significant) }\end{array}$ & $\begin{array}{l}10.1 \% \text { vs } 6.2 \% \text { ( } p= \\
\text { not significant) }\end{array}$ & $\begin{array}{l}20.1 \% \text { vs } 12.4 \% \\
(p=\text { not } \\
\text { significant })\end{array}$ \\
\hline $\begin{array}{l}\text { Nagpal, Raghunandan \& } \\
\text { Saili }\end{array}$ & 2009 & 61 & $\begin{array}{l}\text { Oral misoprostol vs } \\
\mathrm{PGE}_{2} \text { gel. }\end{array}$ & $\begin{array}{l}615 \text { mins vs } 1070 \\
\operatorname{mins}(p<0.001)\end{array}$ & $87.1 \%$ vs $83.3 \%$ & 2 vs 1 case & 3 vs 3 cases & & \\
\hline $\begin{array}{l}\text { Malik, Khawaja, Zahid \& } \\
\text { Rehman }\end{array}$ & 2010 & 100 & $\begin{array}{l}\text { Oral misoprostol vs } \\
\text { sublingual } \\
\text { misoprostol }\end{array}$ & & $76 \%$ vs $90 \%(p>0.05)$ & $4 \%$ vs $2 \%(p>0.05)$ & $20 \%$ vs $8 \%(p>0.05)$ & & $\begin{array}{l}12 \% \text { vs } 8 \% \\
(p>0.05)\end{array}$ \\
\hline $\begin{array}{l}\text { Kulhan, Nur; Kulhan, } \\
\text { Mehmet }\end{array}$ & 2019 & 224 & $\begin{array}{l}\text { Oxytocin vs } \\
\text { dinoprostone. }\end{array}$ & & $\begin{array}{l}64.3 \% \text { vs } 47.3 \% \\
p=0.023\end{array}$ & & $\begin{array}{l}35.7 \% \text { vs } 52.7 \%, p= \\
0.015\end{array}$ & & \\
\hline $\begin{array}{l}\text { Kunt, Kanat-Pektas, Gungor, } \\
\text { Kurt, Ozat, Gulerman, ... \& } \\
\text { Omar }\end{array}$ & 2010 & 240 & $\begin{array}{l}\text { Oxytocin vs } \mathrm{PGE}_{2} \\
\text { pessary }\end{array}$ & & & & $\begin{array}{l}25.6 \% \text { vs } 26.3 \% \\
(p=0.81)\end{array}$ & & $\begin{array}{l}16.7 \% \text { vs } 10.8 \% \\
p=0.46)\end{array}$ \\
\hline $\begin{array}{l}\text { Pourali, Saghafi, Eslami } \\
\text { Hasan Abadi, Tara, Vatanchi } \\
\text { \& Motamedi }\end{array}$ & 2018 & 270 & $\begin{array}{l}\text { Oxytocin vs sublingual } \\
\text { misoprostol }\end{array}$ & & & & 24 vs 24 cases & & $\begin{array}{l}2 \text { vs } 2 \text { cases } \\
(p>0.88)\end{array}$ \\
\hline Mahomed, Wild \& Weekes & 2018 & 184 & $\begin{array}{l}\mathrm{PGE}_{2} \text { gel +/- oxytocin, } \\
\text { vs oxytocin only }\end{array}$ & & & & $\begin{array}{l}16.7 \% \text { vs } 19.1 \% ; \mathrm{RR} \\
1.1595 \%(\mathrm{Cl} 0.61- \\
2.13)\end{array}$ & $\begin{array}{l}11.1 \% \text { vs } 11.7 \% \text {; RR } \\
1.0595 \% \mathrm{Cl}(0.47- \\
2.36)\end{array}$ & $\begin{array}{l}13.3 \% \text { vs } 16.0 \% \\
\text { RR } 1.2095 \% \mathrm{Cl} \\
(0.59-2.41)\end{array}$ \\
\hline $\begin{array}{l}\text { Chaudhuri, Nath Mitra, } \\
\text { Kumar Banerjee, Kumar } \\
\text { Biswas \& Bhattacharyya }\end{array}$ & 2011 & 212 & $\begin{array}{l}\text { PV misoprostol vs } \\
\mathrm{PGE}_{2} \text { gel }\end{array}$ & $\begin{array}{l}16.97 \mathrm{hrs} \text { vs } 16.77 \mathrm{hrs} \\
(\mathrm{p}=0.413)\end{array}$ & & $12.38 \%$ vs $2.94 \%$ & $7.61 \%$ vs $15.3 \%$ & Nil found & $\begin{array}{l}11.42 \% \text { vs } 8.82 \% \\
(p=0.534)\end{array}$ \\
\hline
\end{tabular}


Prostaglandin pessary use was explored with mixed results in the studies by Güngördük et al. (2012), Kulhan and Kulhan (2019), and Kunt et al. (2010). Güngördük et al. (2012) left the pessary in situ for up to six hours prior to commencing IV oxytocin, finding a higher rate of vaginal birth than oxytocin alone, with no difference in other maternal or neonatal outcomes. However, oxytocin alone was better tolerated by women, with less gastrointestinal upset and fewer incidences of maternal pyrexia. Kulhan and Kulhan (2019) reported a higher rate of vaginal birth following IV oxytocin (64.3\%) rather than dinoprostone pessary $(47.3 \%, p=0.023)$, and commented that dinoprostone pessaries were inefficient where the goal is vaginal birth within 24 hours of induction. The study by Kunt et al. (2010) left pessaries in situ for up to 12 hours prior to starting IV oxytocin, or IV oxytocin commenced immediately. This study found a reduction of the duration of labour in the immediate oxytocin group but had a high rate of neonatal admissions to SCBU for both groups (16.7\% and $10.8 \%)$. This may be associated with practices undertaken, e.g. hourly vaginal examination, and replacement of the $\mathrm{PGE}_{2}$ pessary 'if needed', (Kunt et al., 2010).

Prostaglandin gel was regarded positively by Mahomed, Wild, and Weekes (2018) with women randomised to receive up to three doses of vaginal $\mathrm{PGE}_{2}$ gel, with management following the NICE (2014) IOL Guideline, or to receive IV oxytocin alone. They found primiparous women given $\mathrm{PGE}_{2}$ gel needed fewer interventions for fetal heart rate abnormalities than those given oxytocin ( $2 \%$ vs $18.5 \%$, RR 9.25; $95 \% \mathrm{Cl} 1.23$ to 69.8 ). More than half of all women given $\mathrm{PGE}_{2}$ gel did not need oxytocin, particularly multiparous women (33.3\% vs $87.2 \%, \mathrm{RR} 2.61 ; 95 \% \mathrm{Cl} 1.65$ to 4.14 ) but also primiparous women (58\% vs 88.9\%, RR 1.53; $95 \% \mathrm{Cl} 1.19$ to 1.98$)$.

Misoprostol was used in six studies in this group. Three studies focused on comparing misoprostol to $\mathrm{PGE}_{2}$ gels or pessaries but found no clear differences in outcomes (Bricker et al., 2008; Chaudhuri, Nath Mitra, Kumar Banerjee, Kumar Biswas, \& Bhattacharyya, 2011; Nagpal, Raghunandan, \& Saili, 2009). Maternal tolerability in favour of misoprostol was found by Chaudhuri et al. (2011) and Nagpal et al. (2009), but linked to maternal gastrointestinal upset and pyrexia by Bricker et al. (2008) and Pourali et al. (2018).

Route of administration for misoprostol was the focus of three studies, with comparison of oral to sublingual (Malik, Khawaja, Zahid, \& Rehman, 2010); sublingual misoprostol to IV 
oxytocin (Pourali et al., 2018); and oral misoprostol to IV oxytocin in the MisoPROM study (Mozurkewich et al., 2003). The oral route emerged as superior in the Malik et al. (2010) study, with shorter labours, more vaginal births, less IV oxytocin augmentation and fewest doses needed to effect delivery - however the number of participants in this study was too low to achieve statistical significance for any result. Sublingual misoprostol administration was compared to IV oxytocin in the heavily flawed study by Pourali et al. (2018). This study appears to have allocated women to treatment arms based on the time they presented to hospital.

The MisoPROM RCT compared oral misoprostol to IV oxytocin and was referenced by many other studies in this field. Outcomes in this study were equivalent for time to birth, caesarean births and maternal and neonatal morbidity, while women given misoprostol were less likely to have a postpartum haemorrhage ( $1.9 \%$ vs $6.2 \%, p=0.05)$.

Mechanical methods of cervical dilatation were compared by the remaining two studies. Balloon catheters placed above and below the cervix versus IV oxytocin were found to increase the risk of caesarean birth, chorioamnionitis and neonatal SCBU admission (Cabrera et al., 2016). Laminaria tent use did not have any significant benefits, and management of this study was complicated by discretionary clinician use of vaginal prostaglandin for some participants (Kurasawa et al., 2014).

Comparison of these 12 studies into methods of induction for women with PROM did not identify any one method as being either safer or more effective than IV oxytocin alone, though oral misoprostol and vaginal $\mathrm{PGE}_{2}$ gel may be viable options for consideration.

\subsubsection{Intravenous antibiotic prophylaxis following PROM}

Recently, research has focused on the timing of IAP administration following PROM to reduce the risk of GBS transmission to the neonate. Three articles included here each differed in their methodology and research questions. A fourth article compared morbidity outcomes for neonates born in one hospital over a 10 year period, based on whether mothers were given IAP (see Table 2-4).

Nabhan, Elhelaly, and Elkadi (2014) undertook a randomised-controlled trial which used double-blinding of IAP or placebo in 1640 women. Women were included if they presented 
with PROM of less than 12 hours, though the timing of the first dose of IAP is not stated. At 12 hours post ROM, women either had labour induced, or the baby was delivered by caesarean birth. Exclusion of women with latency of over 12 hours and expedited births may have limited findings, and no differences were found in maternal or neonatal outcomes other than an increased rate of caesarean birth for women given IAP. The authors concluded that IAP is not of benefit in actively managed labour following PROM, though this statement assumes prompt interventions are applied (Nabhan et al., 2014).

Passos et al. (2012) conducted a randomised-controlled trial with no blinding other than the paediatrician assessing neonates for sepsis. Women presenting with PROM of under 12 hours and a negative GBS culture at 35-37 weeks were given either IAP or placebo. Induction of labour was applied based on clinical decision-making and used in $61 \%$ of the IAP group and $58 \%$ of the control group.

\section{Table 2-4 Intravenous antibiotic prophylaxis}

\begin{tabular}{|c|c|c|c|c|c|c|c|c|c|c|c|c|}
\hline Author & Year & $n=$ & Method & $\begin{array}{l}\text { Duration of } \\
\text { labour } \\
\text { (hours) }\end{array}$ & $\begin{array}{l}\text { Vaginal } \\
\text { birth }\end{array}$ & $\begin{array}{l}\text { Caesarean } \\
\text { birth }\end{array}$ & Chorioamnionitis & Endometritis & $\begin{array}{l}\text { Neonatal } \\
\text { sepsis }\end{array}$ & $\begin{array}{l}\text { Neonatal } \\
\text { antibiotics } \\
\text { given }\end{array}$ & $\begin{array}{l}\text { Neonatal } \\
\text { CRP }\end{array}$ & $\begin{array}{l}\text { Admission to } \\
\text { SCBU/NICU }\end{array}$ \\
\hline $\begin{array}{l}\text { Barišić, Mandić, } \\
\text { Tomić, Zovko, } \\
\text { Novaković, Barišić, } \\
\text {... \& Novaković }\end{array}$ & 2017 & 144 & $\begin{array}{l}\text { IAP given within } 6 \\
\text { hours of PROM vs } \\
\text { IAP given after } 6 \\
\text { hours of PROM }\end{array}$ & $\begin{array}{l}\text { mean } 5.0 \text { vs } \\
16.0 \\
(p<0.001)\end{array}$ & $\begin{array}{l}76.9 \% \\
\text { vs } \\
73.6 \%\end{array}$ & $\begin{array}{l}7.7 \% \text { vs } \\
11.3 \%\end{array}$ & $3.47 \%$ vs $9.72 \%$ & & & $\begin{array}{l}15.4 \% \text { vs } \\
45.3 \% \\
(p<0.001)\end{array}$ & $\begin{array}{l}3.0 \pm 2.9 \\
\mathrm{mg} / \mathrm{l} \text { vs } 6.1 \\
\pm 7.3 \mathrm{mg} / \mathrm{l} \\
(p<0.001)\end{array}$ & $\begin{array}{l}8.8 \% \text { vs } \\
11.3 \% \\
(p=0.841)\end{array}$ \\
\hline $\begin{array}{l}\text { Nabhan, Elhelaly, } \\
\text { Elkadi }\end{array}$ & 2014 & 1640 & $\begin{array}{l}\text { Single dose of IAP vs } \\
\text { placebo on } \\
\text { admission }\end{array}$ & & & $\begin{array}{l}20.1 \% \text { vs } \\
14.9 \% \\
(p=0.006)\end{array}$ & $\begin{array}{l}2.6 \% \text { vs } 2.3 \%, \mathrm{RR} \\
1.11,95 \% \mathrm{Cl}, \\
(0.60-2.04)\end{array}$ & $\begin{array}{l}0.6 \% \text { vs } \\
0.2 \%, \mathrm{RR} \\
2.5,95 \% \mathrm{Cl} \\
(0.49-12.85)\end{array}$ & $\begin{array}{l}4.1 \% \text { vs } \\
2.9 \%, \mathrm{RR} \\
1.42,95 \% \mathrm{Cl} \text {, } \\
(0.85-2.37)\end{array}$ & & & $\begin{array}{l}5.2 \% \text { vs } \\
4.5 \%, \mathrm{RR} \\
1.16,95 \% \mathrm{Cl}, \\
(0.76-1.78)\end{array}$ \\
\hline $\begin{array}{l}\text { Passos, Cardoso, } \\
\text { Coelho, Graça, } \\
\text { Clode \& Mendes da } \\
\text { Graça }\end{array}$ & 2012 & 161 & $\begin{array}{l}\text { IAP given vs IAP not } \\
\text { given, to women } \\
\text { presenting with ROM } \\
\text { of }>12 \text { hours }\end{array}$ & $\begin{array}{l}17.4+/-8.4 \\
(4.8-50.3) \text { vs } \\
17.3+/-7.9 \\
(3.8-38)\end{array}$ & $\begin{array}{l}78.2 \% \\
\text { vs } \\
80.7 \%\end{array}$ & $\begin{array}{l}21.8 \% \text { vs } \\
19.3 \%\end{array}$ & $\begin{array}{l}2.6 \% \text { vs } 10.8 \% \text {, } \\
\text { RR } 0.9295 \% \mathrm{Cl}, \\
(0.84-0.99) \\
(p=0.037)\end{array}$ & & $\begin{array}{l}3.8 \% \text { vs } 6 \% \\
(p=0.375)\end{array}$ & & & \\
\hline $\begin{array}{l}\text { Geethanath, Ruppa } \\
\text { Mohanram; } \\
\text { Ahmed, Imran; Abu- } \\
\text { Harb, Majd; } \\
\text { Onwuneme, Chike; } \\
\text { McGarry, Kenneth; } \\
\text { Hinshaw, Kim }\end{array}$ & 2019 & 29 & $\begin{array}{l}\text { Observational study } \\
\text { of neonates with } \\
\text { EOGBS, either not } \\
\text { given or given IAP for } \\
\text { PROM of }>18 \text { hours }\end{array}$ & & & & & & $\begin{array}{l}0.86 / 1000 \text { vs } \\
0.35 / 1000 \\
\text { EOGBS }\end{array}$ & & & \\
\hline
\end{tabular}

This study monitored women heavily, with full blood count and C-Reactive Protein blood ${ }^{11}$ tests completed twice daily until birth, and continuous cardiotocograph recording from

\footnotetext{
${ }^{11}$ A C-reactive protein test checks for a protein which increases when there is inflammation or infection (Health Navigator New Zealand, 2020)
} 
admission. Maternal infection was reduced with the use of maternal IAP ( $2.6 \%$ vs $13.2 \%$, RR $0.89 ; 95 \% \mathrm{Cl} 0.81$ to 0.98 ), and no infections occurred in the $27 \%$ of women who birthed within 12 hours of ROM. Fewer neonates born to women treated with IAP developed infection, though statistical significance was not achieved. This study concluded IAP is of benefit for women electing either expectant management or induction of labour for PROM (Passos et al., 2012).

A randomised-controlled trial of 144 women considered results for women and babies, where the women were routinely given IAP either within the first six hours after ROM, or later. Neonates born to women who had received IAP within six hours post-PROM were less likely to be given antibiotics themselves, compared to those where maternal IAP had commenced more than six hours post-ROM ( $15.4 \%$ vs $45.3 \%, p=0.001)$. The rate of neonatal treatment was concerningly high with 38 neonates ( $26.4 \%$ overall) given IV antibiotics, though an unspecified number were treated solely due to meconium-stained liquor (Barišić et al., 2017).

Geethanath et al. (2019) undertook an observational study of babies with EOGBS and compared neonatal morbidity and mortality outcomes after the introduction into practice of giving maternal IAP (after 18 hours of PROM). The incidence of EOGBS more than halved, and mortality reduced from $12.5 \%$ to $7.5 \%$. This study found that of the neonates who developed EOGBS, those neonates born without maternal IAP cover had an overall morbidity incidence of $31.25 \%$, including cerebral palsy (18\%), hearing loss (18\%), ocular damage (12.5\%) and cranial diabetes insipidus (12.5\%), while the neonates with EOGBS subsequent to maternal IAP did not display any of these morbidities.

\subsection{Discussion}

Differences between duration and definition of expectant management, timing of induction of labour processes, and method and route of induction processes made clear comparisons of many outcomes difficult. Few provided reliable evidence for practice, and the scattering of approaches may reflect localised interests driving research projects during the $21^{\text {st }}$ century. Even the Cochrane systematic review by Middleton et al. (2017), which reviewed 
23 trials from 1992 to 2015, identified no differences predictive of caesarean birth, serious maternal morbidity or mortality, or neonatal mortality.

Duration of labour was commonly reported as prolonged where women did not spontaneously establish into labour within a set timeframe. The 96-hour expectant management period used by Hannah et al. (1996) remains an outlier, with all other publications using an expectant management period of 12-24 hours. Both Li et al. (2011) and Namli Kalem et al. (2017) argued that due to an inherent pathology associated with PROM, if labour does not follow spontaneously within 12 hours, birth should be expedited by either immediate induction of labour or caesarean to avoid poor maternal and neonatal outcomes. Support for planned early birth, defined as 'immediate intervention or intervention within 24 hours', also comes from Middleton et al. (2017, p. 1), who found labour duration was shorter for women managed actively and duration of hospital admission was shorter.

It is unclear from this review whether mode of birth is affected by how PROM is managed. Vaginal, instrumental, and caesarean birth outcomes were all presented with conflicting findings, and in many cases with no significant $p$ values. The largest, and well-designed study reported little variation between groups regarding caesarean births (ranging from 9.6\% to $10.9 \%$ ), with no statistical significance (Hannah et al., 1996).

Postpartum haemorrhage was positively linked to PROM in two studies, however the duration of PROM needed to increase the risk of PROM was only considered by one study, which logged the incidence of PROM in 2-hourly time intervals (Tran et al., 2008). Most studies did not include postpartum haemorrhage as a key outcome, so the evidence is minimal. Middleton et al. (2017) stated there is no link, however only compared three small studies (two of which were very dated, and none of which are included in this literature review).

Chorioamnionitis and endometritis increased in incidence as duration from ROM increased. Neonatal morbidity also increased with increasing duration from ROM to active labour, with predictive factors of maternal clinical chorioamnionitis, positive GBS status and increasing numbers of vaginal examinations (Li et al., 2011; Seaward et al., 1998). 
Most studies in this review routinely gave IAP on admission, and few commented on the effect. The few studies providing comparisons based on the timing of IAP administration also included other interventions to influence the duration of labour, which reduces the ability to analyse outcomes based on IAP use alone. In New Zealand, planned early birth is a recommendation (Clinical Guidelines Panel, 2019; Middleton et al., 2017), but women who are otherwise at low risk for GBS transmission are offered 18-24 hours (or more) of expectant management, therefore early administration of IAP to all women does not reflect the variations in New Zealand consent-based clinical practice. In the Middleton et al. (2017) review, women with a planned early birth had a reduced risk of maternal infection (either chorioamnionitis or endometritis) (average RR $0.49 ; 95 \% \mathrm{Cl} 0.33$ to 0.72 ), and their neonates also had a lower risk of probable or definite sepsis ( $\mathrm{RR} 0.73 ; 95 \% \mathrm{Cl} 0.58$ to 0.92 ). Babies born to women in the early birth groups were less likely to be given antibiotics (RR 0.61; $95 \% \mathrm{Cl} 0.44$ to 0.84 ) and less likely to be admitted to neonatal special care units (RR 0.75; $95 \% \mathrm{Cl} 0.66$ to 0.85$)$. Reflecting on their findings, the authors noted that their review supports planned early birth primarily to avoid the need to administer unnecessary antibiotics to both mothers and babies, however the quality of evidence reviewed was generally poor (Middleton et al., 2017).

Comparisons of methods of induction of labour following PROM were the focus of a very significant proportion of research related to the management of PROM, however variation of induction methods and timing in the studies made it not possible to make direct comparisons or identify consistent findings for any key outcomes. Oral misoprostol may be a viable option for further consideration, however, the MisoPROM trial was ceased prematurely, due to recruitment difficulties following the publication of a letter written by the US Medical Director of the manufacturer, Searle, warning against the use of misoprostol for the induction of labour (Mozurkewich et al., 2003). Despite this, misoprostol remains in common use in many countries due to efficacy, simple transport and storage, low cost and ease of administration (Chaudhuri et al., 2011; Nagpal et al., 2009). Misoprostol was not included as an active management option in the RANZCOG (2017) consensus statement for term PROM, but has recently been supported for general use by the Induction of Labour in Aotearoa New Zealand clinical practice guideline, although it is not mentioned in connection with PROM (Clinical Guidelines Panel, 2019). 
The RANZCOG (2017) term PROM consensus statement heavily references the Middleton et al. (2017) review and the Hannah et al. (1996) study, with the remaining references a mix of current and older material. Support is given for the use of expectant management, assuming no digital vaginal examinations, for up to 24 hours in "...highly selected and well supervised cases" (RANZCOG, 2017, p. 6), although there is benefit seen from IAP from 12 hours post ROM. Expectant management is defined as requiring 4-hourly maternal temperature checks, evaluation of vaginal loss and regular assessment of fetal well-being. However, there is no recommendation adopted by RANZCOG to reflect the conclusion of Middleton et al. (2017) that women may prefer immediate induction of labour to avoid IAP or neonatal antibiotic administration, and research would be needed to identify what is occurring in practice throughout New Zealand.

\subsection{Issues of Concern in the Research}

The greatest challenge in writing this review was comparing concrete outcomes, e.g. mode of birth, where management of labour and definitions of management events varied significantly. For example, 'expectant management' is not one commonly held concept, with duration, location and clinical assessments being inconsistent between studies, and at times entirely different to what New Zealand-based practitioners would identify as expectant management. Other issues included use of digital vaginal examination on admission, and the apparent lack of women's engagement with decision-making.

Digital vaginal examination on admission was used in many of the included studies but is discouraged in New Zealand practice until spontaneous active labour or induction of labour, to reduce the risk of ascending infection. Of the 32 clinical studies included in this literature review, 23 stated they used vaginal examination at admission, which suggests that international practice varies significantly, and differs to that in New Zealand. Two studies used hourly vaginal examinations to assess progress (Kunt et al., 2010; Mozurkewich et al., 2003), and even in the Hannah et al. (1996) study almost $37 \%$ of participants across all groups received digital vaginal examination prior to labour establishing, though who had them is not explored (Hannah et al., 1996; Radoff, 2014). However, several studies found there were benefits of vaginal prostaglandins for women with low Bishop Scores, in advancing the progress of labour and reducing the incidence of caesarean birth (Güngördük 
et al., 2012; Larrañaga-Azcárate et al., 2008; Saccone \& Berghella, 2015). The balance between avoiding unnecessary vaginal examination versus the benefit of cervical ripening was an aspect of managing PROM discussed by Middleton et al. (2017) and RANZCOG (2017), and is ripe for further discussion in the New Zealand setting.

The Hannah et al. study had limitations which are worth revisiting. GBS was found on admission swab culture in $9.1 \%$ to $11.8 \%$ of women, and IAP was given to $10 \%$ of women, but it was not reported which women received it (Radoff, 2014; Seaward et al., 1998). Chorioamnionitis was defined solely by a maternal temperature of $37.5^{\circ} \mathrm{C}$ on two occasions or $38^{\circ}$ on one occasion, which contributed to the high incidence of chorioamnionitis reported in this study, despite a lack of confirmation by cultures. The definition of expectant management appeared to differ in various centres participating in the study, with about $60 \%$ of women in the expectant management groups awaiting labour in their respective hospitals (Hannah et al., 1996). It is logical to assume assessments, monitoring and interventions will have been more frequent than if they had returned to their homes but this was not reported.

Maternal perspectives were sought in only two of the included studies. Women in the Hannah et al. (1996) study were asked to rate their satisfaction with their treatment group; however the publication only stated that women were 'less dissatisfied' with active management compared to those given expectant management. Mahomed et al. (2018) also found women given immediate IOL with oxytocin were the most satisfied with the time it took to establish labour, though they reported more side effects of their treatment. An older study not included in this review (Selmer-Olsen, Lydersen, \& Mørkved, 2007) found women given acupuncture during expectant management of up to 48 hours viewed it positively, i.e. they liked it better than nothing, despite no effect on labour duration or birth outcomes. It is probable that maternal satisfaction with PROM management includes aspects of perceiving that care is being given, as well as participation in decision-making. In New Zealand women have the right to informed consent and to participate in decisionmaking processes (Health and Disability Commissioner, 1996) therefore consumer input into the management of PROM at term would be expected and provides useful insights. 


\subsection{Limitations of this literature review}

Due to the volume of literature available, this literature review focused on reviewing only clinical trials and retrospective reviews with direct relevance to the management of PROM at term and the related outcomes. Eliminating systematic reviews, which typically included the same and older studies, made comparison simpler, but may have assisted in summarising existing publications.

GBS and EOGBS are closely related topics to PROM, however this review excluded literature related solely to GBS/EOGBS, to keep the focus on PROM. This review also did not consider use of specific antibiotics, as culture and sensitivity results vary between countries and alter over time. Deeper discussion of GBS management would have extended the scope of this review extensively, and led into further topics such as potential vaccination and improved clinical diagnostic tools, such as rapid identification of GBS by polymerase chain reaction (PCR) (Melin, 2011).

\subsection{Gaps in published research}

There are very large gaps in current research for modern, well-constructed randomisedcontrolled trials to reconsider management of PROM, with outcomes extending long-term into childhood (Middleton et al., 2017). The benefits versus risks of expectant and active management strategies for PROM need to be reconsidered in current practice environments and include accurate intrapartum testing for GBS. Future research needs to consider women's views of management following PROM, and research must also demonstrate informed consent and women's participation in decision-making. There appears to be a gap in the research for a study to consider the possible benefit of immediate induction of labour as an option, if women would prefer to avoid IAP. There are current studies investigating the long-term outcomes of IAP on the maternal and neonatal microbiome, which may be of influence in the future and could lead to benefit versus risk comparisons and ethical discourse.

A GBS vaccine and rapid PCR testing for GBS will require research and are likely to alter practice and increase women's options. Rapid PCR testing for GBS is poised to alter management of GBS in labour by identifying GBS status in under 60 minutes, and is the 
subject of current research, notably the UK-wide GBS3 study which will compare risk-based GBS management versus universal screening of GBS in labour by rapid PCR testing versus standard culture and sensitivities (Nottingham Clinical Trials Unit, 2020). Locally conducted studies would assess the feasibility of using PCR testing in labour and potentially lead to a change in practice.

\subsection{Conclusion}

The key findings from this literature review are that despite the high volume of research around the topic of PROM at term, high quality research is lacking. Recent research typically lacks statistical power to inform evidence-based practice and is difficult to generalise to the New Zealand maternity system.

Vaginal $\mathrm{PGE}_{2}$ and oral prostaglandins are worth further exploration for women with a Bishop Score of four or less, and may reduce the need for oxytocin, reduce the duration of labour, and increase the rate of vaginal births. Alternatively, immediate IOL with oxytocin is supported as reducing the number of vaginal examinations, shortening the time to onset of labour, and shortening the duration of labour, as well as reducing the use of IAP and neonatal antibiotics. The Hannah, et al., (1996) study continues to provide a foundation for much current guidance, and has sustained the NDHB PROM policy, however is also used by other authors to justify differing perspectives (Radoff, 2014). Research into the long term effects of active management and IAP are lacking (Middleton et al., 2017), and practice in the future is likely to alter if either a vaccine for GBS is developed or rapid PCR testing is adopted (Darlow et al., 2015; Melin, 2011).

The next section will cover the methodology and method used for data collection and analysis in this thesis. 


\section{$3 \quad$ Methodology}

Chapter 3 presents the research methodology, and describes the methods undertaken to investigate and analyse the outcomes of the NDHB PROM policy. A retrospective process and outcome evaluation used a clinical records review to collect and analyse quantitative demographic, antenatal, intrapartum, birth and neonatal outcome data, with comparison to NDHB and New Zealand-wide figures. The aim of this study was to evaluate and describe the processes of care, and outcomes for women birthing at NDHB facilities following PROM (Posavac \& Carey, 2007). The research asked several questions:

1. Was the NDHB PROM policy applied in practice (process evaluation)?

2. Did this policy meet the needs of birthing women experiencing PROM (outcome evaluation)?

3. Was/is the current policy safe for women and babies? (outcome evaluation) (Mertens \& Wilson, 2012).

Cases included in this study were all women (and their babies), experiencing spontaneous PROM of 18 hours or more with subsequent birth at any NDHB facility, over a one-year period from 1 June 2018 to 31 May 2019. Analysis included inductive reasoning and descriptive data analysis to look for patterns and relationships within the data available.

\subsection{Discussion of the Quantitative Approach}

A quantitative approach was used to assess the effect the NDHB PROM policy had on outcomes for birthing women and their babies (Bryman, 2016). Like any maternity service, NDHB birthing outcomes result from multifactorial conditions. Pragmatic evaluation of this program of care was intended to shed light on whether this policy contributes to local outcomes, such as the low rate of caesarean birth, or the high rate of neonatal admission to SCBU found at NDHB (2017). Quantitative data collection and analysis also enabled descriptive comparison by factors such as ethnicity or maternal smoking, to assess equity of access to services or causative factors in poor outcomes. 
Methodology

Qualitative data collection and analysis was not used as in practice it could relate to only a small number of experiences and may not have been reflective of broader realities or all affected women in vivo. Surveying or interviewing maternity care providers would have supplied some experiences, but also opinions and sources of bias, without providing a background of statistical outcome data. A mixed methods study could have incorporated the two approaches, however, would have been unwieldy within the scope of a Master's thesis (Bryman, 2016).

Exploratory approaches are best suited to areas about which there is little currently known. There is extensive research published regarding PROM (Middleton et al., 2017), so this is not the best fit for this project. Explanatory approaches seek to explain why events or processes occur the way they do and seek a depth of information (Babbie, 2008), and this approach is better suited to this project.

Descriptive studies aim to utilise what can be observed or recorded - in this case what can be found in clinical notes and outcomes records - and to describe what can be seen or found in that data. A descriptive approach does not require the formal statement of one or more hypotheses prior to design, though the researcher may be able to anticipate probable questions that may arise (Bryman, 2016). A descriptive approach includes the ability to attempt to identify relationships within the data and can be extended into discussing why outcomes or circumstances are what they are, which was helpful in this study in discussing research findings (Babbie, 2008). A descriptive, explanatory approach fits well within the evaluation model and is the best fit for this project.

\subsection{Methodology: Evaluation Research}

The overall aim of this research project was to provide an evaluation of the clinical decisionmaking and application of policy, birthing outcomes and events experienced by women and their babies birthing within NDHB following PROM. Evaluation is a systematic quality improvement process used to assess the value or merits of an organisational program (also called an evaluand), and can be used in health, education, business, or government. Evaluation can be used to examine a variety of factors, including that a service exists and the degree of utilisation, what aspects of a service or program work well, most efficiently or are most effective, areas for improvement, and cost-benefit analysis. Evaluation compares 
Methodology

what is occurring against what is expected, and functions as a pragmatic quality improvement feedback loop. Evaluation is broad and necessarily includes context and can be used to compare outcomes external to the programme or organisation (such as with unaffected women or women birthing in other DHBs). In comparison, audit is narrow and focuses only on specific local measures with a strong focus on compliance with service standards, and is more typically performed in-house. Evaluation can be conducted by either an internal or external evaluator (Ekart, 2014; Mertens \& Wilson, 2012; Posavac \& Carey, 2007).

The Aotearoa New Zealand Evaluation Association (ANZEA) recommend four framing principles for evaluation projects (Table 3-1), which were applied and reflected on regularly by the researcher during this project. These principles have been grounded in Māori, Pākehā and Pasifika values for use in the New Zealand context.

Table 3-1 ANZEA Evaluation Standards

\begin{tabular}{|c|c|c|c|}
\hline $\begin{array}{l}\text { Respectful, } \\
\text { meaningful } \\
\text { relationships }\end{array}$ & Ethic of care & $\begin{array}{l}\text { Responsive } \\
\text { methodologies } \\
\text { and trustworthy } \\
\text { results }\end{array}$ & $\begin{array}{l}\text { Competence and } \\
\text { usefulness }\end{array}$ \\
\hline $\begin{array}{l}\text { - Relationships } \\
\text { - Involvement } \\
\text { - Communication } \\
\text { - Negotiated } \\
\text { accountabilities, } \\
\text { resources and } \\
\text { governance } \\
\text { - Self-determination }\end{array}$ & $\begin{array}{l}\text { - Care } \\
\text { - Respect } \\
\text { - Inclusion } \\
\text { - Protection } \\
\text { - Reciprocity }\end{array}$ & $\begin{array}{l}\text { - Responsive } \\
\text { - Systematic and } \\
\text { robust } \\
\text { - Evaluative validity } \\
\text { and reasoning } \\
\text { - Multicultural } \\
\text { validity } \\
\text { - Transparent }\end{array}$ & $\begin{array}{l}\text { - Professional } \\
\text { competence } \\
\text { - Independence and } \\
\text { interdependence } \\
\text { - Project } \\
\text { management } \\
\text { - Usefulness } \\
\text { - Evaluation } \\
\text { accountability }\end{array}$ \\
\hline
\end{tabular}

Source: Social Policy Evaluation and Research Unit (2015, p. 7).

This research project took a quality improvement approach and focused on evaluating the contribution of the NDHB PROM policy, (the program process), and evaluation of the outcomes of the program. A sample large enough to achieve statistical significance and a $p$ value of $<0.5$ within the NDHB population was used. Data were sufficient to allow for comparison with the outcomes of that population, versus the wider New Zealand population. As the NDHB policy differs significantly from the remainder of New Zealand practice, this one-off study investigated the effect of an existing treatment program 
Methodology

(extended expectant management of over 24 hours) on women. By using a large sample and a consistent data collection tool, the results could be replicated. The same outcome measures were applied where possible to the wider NDHB and COMCORD data collection, making the results generalisable (Brophy, Snooks, \& Griffiths, 2008).

\subsection{Method: Clinical records review and database comparison}

Engagement with NDHB Maternity service managers was undertaken to aid in research design, as stakeholder involvement is a key factor in evaluation, and the end-product of evaluation is only one factor in policy development (Mertens \& Wilson, 2012; Posavac \& Carey, 2007). Consultation confirmed the purpose, aims and objectives of the project, clarified the practical processes for data collection, and confirmed the desired outcome measures (Ashmore, Ruthven, \& Hazelwood, 2011a). Planning with stakeholders also acknowledged the data belongs to NDHB, both in terms of being a tangible product which could be utilised by the DHB in their routine clinical quality improvement cycle; and a taonga ${ }^{12}$, a product reflecting the unique character of NDHB and the local community. The NDHB in-house audit guideline written by Ekart (2014) was referred to as a local guide to understand organisational structure and maximise quality assurance (Ashmore et al., 2011a). The NDHB maternity management team agreed it was desirable to have a robust data set that could be compared with wider DHB and national statistics, to identify whether the existing NDHB PROM policy serves the NDHB birthing community well. NDHB managers and/or staff may choose to utilise recommendations in the design, implementation and maintenance of any improvements processes indicated by the results (Ashmore et al., 2011a).

Monetary costs of the data collection phase of the project were low to NDHB, including a small amount of staff time assisting with identifying and locating files to review, providing a workspace for the researcher, and providing parking to the researcher free of charge. Researcher costs included travel time and expenses to visit Whangarei Base Hospital, costs

12 Treasure, property, possession 
Methodology

of purchasing a laptop, and time to develop and test a data collection tool. Researcher costs were covered by a small financial grant, with thanks to Graduate Women Wellington.

Mertens and Wilson (2012, p. 282) state that an outcome evaluation requires the evaluator to answer six questions in the planning phase, which are presented in Table 3-2.

Table 3-2 Outcome evaluation

\begin{tabular}{|c|c|}
\hline Evaluation planning question & This evaluation \\
\hline $\begin{array}{l}\text { Who is the target population and } \\
\text { are there any subgroups of that } \\
\text { population? }\end{array}$ & $\begin{array}{l}\text { All women birthing with PROM within NDHB } \\
\text { facilities over a 1-year period. } \\
\text { Subgroups included: wāhine Māori, women who } \\
\text { smoke, women pregnant with babies who were } \\
\text { small for gestational age. }\end{array}$ \\
\hline $\begin{array}{l}\text { What outcomes for the target } \\
\text { population and subgroups are } \\
\text { sought because of this evaluation? }\end{array}$ & $\begin{array}{l}\text { - Women are given appropriate care. } \\
\text { - Women are involved in decision-making } \\
\text { about their care. } \\
\text { - Women and babies have safe birthing } \\
\text { outcomes. } \\
\text { - Care provided is equitable within NDHB and } \\
\text { nationally. }\end{array}$ \\
\hline $\begin{array}{l}\text { How will you measure the } \\
\text { achievement of those outcomes? }\end{array}$ & $\begin{array}{l}\text { The data collection tool collated clinical data for } \\
\text { quantitative analysis; bivariate analysis identified } \\
\text { any issue of inequity. }\end{array}$ \\
\hline $\begin{array}{l}\text { What practical matters need to be } \\
\text { allowed for in data collection - such } \\
\text { as cost, time, access }\end{array}$ & $\begin{array}{l}\text { Researcher costs of mileage to NDHB facilities; time } \\
\text { to develop data collection tool and review clinical } \\
\text { data; equipment including laptop. } \\
\text { NDHB costs of staff time to locate files and assist } \\
\text { with queries; access to staff areas, databases, and } \\
\text { files. }\end{array}$ \\
\hline
\end{tabular}




\begin{tabular}{|c|c|}
\hline $\begin{array}{l}\text { How will you use the results from } \\
\text { this evaluation? What if the results } \\
\text { are unfavourable or unexpected? }\end{array}$ & $\begin{array}{l}\text { The results were used to compare outcomes for } \\
\text { women experiencing PROM, with other women } \\
\text { birthing within NDHB and in wider New Zealand. } \\
\text { Results were provided to NDHB, whether } \\
\text { favourable, unfavourable, or unexpected, and may } \\
\text { be used in quality improvement processes. Results } \\
\text { were presented with respect, and language of } \\
\text { deficit was avoided in writing the thesis. }\end{array}$ \\
\hline $\begin{array}{l}\text { What are your performance } \\
\text { targets - how will you know your } \\
\text { evaluation has met your targets? }\end{array}$ & $\begin{array}{l}\text { The performance targets were: } \\
\text { - Adequate sample numbers } \\
\text { - Solutions Plus and COMCORD data available } \\
\text { and suitable for comparison } \\
\text { - Statistical analysis appropriate and accurate }\end{array}$ \\
\hline
\end{tabular}

\subsection{Ethical and locality approval}

The Health and Disability Ethics Council screened the Scope of Review and responded (9 November 2018) that as this study is observational only and at the level of master's study an application for full approval was not required. A copy of the approval letter from the Health and Disability Ethics Council is included in Appendix 3. The VUW Human Ethics Committee reviewed the application and approved it following minor changes (20 December 2018, reference 0000026842, Appendix 4). Ethical conduct of studies is essential in midwifery and the following discussion outlines how such matters were addressed. NDHB granted locality approval for this research to be completed (see Appendix 5).

To protect anonymity, non-identifiable numbers were used for reviewed records, and identifying features were not recorded other than NHI numbers used to locate files. No identifying data is reported. Confidentiality was protected through use of good data security practices, including password protection, and hard copy data stored in a lockable filing cabinet. Hard copy data will be destroyed 5 years after publication of the thesis. Electronic data will also be securely deleted in 5 years. Privacy was protected by following 
Methodology

correct protocols to access health records, and utilising NDHB office space for data collection activities. Researcher safety was protected as my partner and supervisors were aware of my itinerary when visiting NDHB facilities. It was unlikely this study would cause harm, excepting the potential to harm the reputation of NDHB, however NDHB was supportive of this research project and had design input. Informed consent by individual women was not required under the Privacy Act 1993 Principle 10(1)f (Ministry of Justice, 1993), however VUW Human Ethics Committee approval was gained, as it is intended to publish and/or present findings publicly. Cultural safety was important in relating to NDHB staff on site or elsewhere, and in interpreting and presenting this research. Appropriate consultations, with respect to the Treaty of Waitangi and to local practices occurred prior to commencing data collection.

\subsection{Treaty of Waitangi issues and Māori consultation}

I acknowledge my Pākehā background and ongoing desire and obligation to consider the Treaty of Waitangi as a guide in considering tikanga Māori. I referred to Te Ara Tika, Guidelines for Māori Research Ethics (Hudson, Milne, Reynolds, Russell, \& Smith, n.d.). My research takes a mainstream approach, but results will be of interest to Māori, and the demographic makeup of the region includes many Mãori as participants. It is hoped that results will promote health equity for wāhine and their pēpi, who represent $48 \%$ of women birthing in NDHB (NDHB, 2017).

Consultation was sought with Māori Advisors at Victoria University of Wellington and NDHB, with the aim of constructive kōrero ${ }^{13}$, and to kia āta-haere ${ }^{14}$. Individuals who contributed to this kōrero were: Meegan Hall, Assistant Vice Chancellor (Mātauranga Māori), of Victoria University of Wellington, Te Ihi Tito, from the Māori Health Directorate of NDHB, and Crete Cherrington, LMC Midwife in the NDHB rohe ${ }^{15}$, and Kuia for NZCOM. Their contribution supported me to view the data and information I accessed as a taonga. I endeavoured to

\footnotetext{
${ }^{13}$ Discussions, negotiations

${ }^{14}$ Proceed with understanding

${ }^{15}$ Area, district
} 
Methodology

kaitiaki ${ }^{16}$ the data by adhering to the Code of Conduct for midwives, by adhering to the laws, regulations and code of New Zealand through good data security practices and using anonymity in data recording (New Zealand College of Midwives, 2015). The ANZEA principles of ethic of care and respectful meaningful relationships are based on Māori and Pasifika values and views (Social Policy Evaluation and Research Unit, 2015). In my analysis and discussion, I tried to uphold whakarangitira ${ }^{17}$ by reflecting sensitively on Māori values, traditions, and outcomes, and avoiding deficit language in my writing. I hope to be able to present my findings at one or more local events, to share findings appropriately with community members. My abstract has been approved for presentation at the next NZCOM Conference (postponed to 2021 due to disruptions resulting from the COVID-19 lockdown in early 2020).

\subsection{Data Collection}

\subsubsection{Data collection tool design}

A data collection tool was designed using Microsoft Word, with a clear process through demographic, antenatal, intrapartum, birth and neonatal data stages of data collection (see Appendix 6). The fields in the data collection tool were identified by reviewing the NDHB PROM policy, and also from evidence-based guidance and literature review material, as well as reviewing a blank clinical notes set produced by the Midwifery and Maternity Providers Organisation (MMPO), and the Referral Guidelines (Ministry of Health, 2012). To contrast outcomes for affected women and their babies to those of unaffected women and those birthing outside of NDHB, demographic data and contextual features also added depth to the data (Ashmore et al., 2011a). The data collection tool pages were each given a unique identifying number per record. Maternal and neonatal NHI numbers were documented together with their allocated identification number in a separate file and used solely during data collection to ensure all files were reviewed and to investigate any queries. The data collection tool itself did not include any identifying data and none has been entered into

\footnotetext{
${ }^{16}$ Custodian, trustee, caregiver

${ }^{17}$ Dignity, honour, respecting of
} 
Methodology

SPSS. Data entry was direct into SPSS and coded into groups as relevant, with numerical values representing data values where possible, to enable statistical comparisons.

\subsubsection{Data sources}

The primary data sources used for the clinical record review were clinical notes and Maternity Plus data of women birthing at term with PROM. Women were identified through a download of a search of the NDHB Maternity Plus data management system conducted by an NDHB data analyst. This process also provided outcome data for women birthing at term without PROM, to provide wider NDHB outcome data. The New Zealand College of Midwives Clinical Outcomes Research Database (COMCORD) provided data relating to nationwide outcomes for women birthing with and without PROM. This data originates from community midwives who use MMPO as their claiming agent (for funding from the Ministry of Health) and includes information from approximately half of all New Zealand births annually (MMPO, 2016).

\subsubsection{Clinical Data Recording and Data Entry}

Where possible, paper-based data recording used the same coding as the eventual electronic data field in SPSS, i.e. numerical, date: time, or yes/no values, or allocated a number to assist with coding processes. In subsequent data analysis, many fields which used numbers (for example ethnicity, mode of birth and breastfeeding status on discharge) were separated into individual yes/no fields so that odds-ratio calculations could occur. Data was entered into SPSS and rechecked for accuracy.

The NDHB Solutions Plus data was supplied as an excel file and converted to SPSS, and the COMCORD data was supplied in SPSS. There were interesting differences in the quality of the data and how outcomes were coded, which will be discussed later in this chapter.

\subsubsection{Clinical records review sample}

The settings for this research were NDHB maternity facilities; predominantly Whangarei Base Hospital but also Bay of Islands Hospital at Kawakawa and Kaitaia Hospital. The maternity facility at Dargaville had no births within the PROM sample, and two births within the NDHB 
Methodology

sample. The clinical records review used a convenience sample of all birthing women, over one year from 1 June 2018 to 3 May 2019, identified as having PROM, within NDHB Maternity facilities: and their babies. The sample size was set as a one-year period based on the following calculation:

2154 births in Northland in the 2016/17 year .

PROM affects $8-10 \%$ of pregnancies, $60 \%$ of which are at term (Ismail \& Lahiri, 2013); therefore $4.8 \%$ to $6 \%$ of all NDHB births.

$$
\left.\begin{array}{l}
2154 \times 0.048=103 \\
2154 \times 0.06=129
\end{array}\right\} \begin{aligned}
& \text { estimated range of term births with } \\
& \text { PROM in NDHB per year }
\end{aligned}
$$

In a population of 2154 , a proportion (incidence of PROM) of 0.048 to 0.06 with a confidence level of $95 \%$ and a confidence interval of $5 \%$ would require a sample size of 68 to 84. A one-year sample, allowing for attrition through birthing either at home or at Rawene, would provide enough data and be comparable to wider NDHB and national statistics (Ashmore, Ruthven, \& Hazelwood, 2011b; Australian Bureau of Statistics, n.d.).

Clinical records sampling reviewed a full one-year cluster of women, as to randomly or systematically eliminate any files risked the inadvertent omission of sentinel events, for example, any admission of a neonate to SCBU with respiratory distress (Babbie, 2008). Sampling from a full year avoided any seasonal effects, such as severe weather events such as flooding, to which Northland is susceptible and which may have affected access to care (Kronast \& Lewis, 2020). The clinical records review could have been conducted over a longer period, such as three years, and randomly sampling every third file - but this offered no apparent advantage over the calendar year sample, and would help prevent individual women from being included in the same study twice, however there was a relatively low chance of this occurring in a one year sample.

\subsubsection{Inclusion criteria}

The inclusion criteria in this study were as follows:

- Women birthing within an NDHB facility at $\geq 37$ weeks gestation. 
Methodology

- Duration from membrane rupture to birth $\geq 18$ hours or coded PROM.

- Live foetus at time of ROM.

Seven women had ROM at less than 37 weeks gestation, but initially used expectant management, generally as inpatients, and subsequently birthed after $\geq 37$ weeks, so were included.

PROM is usually defined as the time from ROM to labour establishing. This study included women with a duration of $\geq 18$ hours from membrane rupture to birth. This is because not all women with ROM establish into labour, and accurately defining established labour can be subjective. In addition, PROM and/or GBS management policies in DHBs around New Zealand recommend the offer of IAP from either 18 or 24 hours for women who are labouring. NDHB recommends IAP from 24 hours (and in labour), however it was relevant to have women with PROM of 18:00 to 23:59 hours included in the sample to increase the ability to generalise findings throughout New Zealand. The duration of 18 hours from ROM and in established labour was also guided by the New Zealand Consensus Guideline by Darlow et al. (2015).

Confirmation of a live foetus at the time of ROM, through maternal report of fetal movements or auscultation of the fetal heart, or at the very least no suspicion/confirmation of fetal demise, was sought to ensure care management was not different due to fetal demise prior to ROM. All births were of live babies and none were excluded on this basis.

An NDHB business analyst provided a download of all births recorded at NDHB facilities at gestations of 37 weeks or more from 1 June 2018 to 31 May 2019. The total number of women included in this sample was 1840 . He applied a search to identify all women with duration of membrane rupture prior to birth of 18 hours or more and identified 133 files to request and review. The NHI numbers were forwarded to NDHB Release of Information staff and the files requested. Due to the identification of date: time data-entry errors in Maternity Plus, it was suspected that some files eligible for inclusion may not have been identified using date: time entries, and subsequently the analyst was requested to repeat the search using clinical PROM coding, which located another 24 files not previously seen. This led to a final number of 157 files reviewed. 
Methodology

\subsubsection{Exclusion criteria}

There were three exclusion criteria as follows:

- Membrane rupture of less than 18 hours prior to birth

- Induction of labour with subsequent prolonged ROM

- Not birthing in an NDHB maternity facility

Exclusion removed 26 files, including 16 where errors in data entry had created false PROM events. Seven women were found to have had an induction of labour and gone on to have prolonged ROM. One file had been coded for PROM due to an antenatal swab found to be positive for GBS, but duration from ROM to birth was only seven hours. Data were collected for these eight files but later excluded from analysis as it was decided they did not meet the definition of PROM.

The final PROM sample is 123 maternal and neonatal records, plus one baby from a twin pregnancy. Only the neonatal data were recorded for Twin Two from this birth record, to avoid counting data from the mother twice. The sample size achieved is within the expected range and exceeds the numbers required for a confidence level of $95 \%$ and a confidence interval of $5 \%$.

Not included were the $6-7 \%$ of births at home (NDHB, 2018a) and $1.4-2.3 \%$ of births at Rawene Hospital (Hokianga Health Enterprise Trust, 2018), due to the impracticality of tracing, consenting and obtaining copies of notes from multiple LMC midwives.

The NDHB data sample has 1717 women who birthed at gestation 37 weeks or more within NDHB facilities from 1 June 2018 to 31 May 2019, with no PROM of $\geq 18$ hours. No further exclusions have been applied to this group, but a small amount of unfeasible data, for example excessive gestations have been deleted, and data has been converted to the correct syntax for SPSS analysis.

The NZCOM COMCORD sample provided 30165 women, however included 1663 records of women who birthed at less than 37 weeks gestation, which have been removed from analysis. This leaves a total sample from COMCORD of 28502 women. Data cleaning was completed by COMCORD prior to supply and the sample was generally accepted as is, 
Methodology

except for the following issues which would have affected calculations through either being implausible, incorrect or extreme outliers:

- Duration of 1st stage of labour contained 1522 entries with negative values, and four entries of duration over one month. All negative time entries and all duration of over 48 hours were removed and formed 'missing' values.

- Duration of 2 nd stage exceeded 720 minutes (12 hours) for 1136 entries, up to 5260346 minutes (over a year). All entries over 720 minutes were removed and formed 'missing' values.

- Duration of 3rd stage had two negative entries and 15 entries significantly beyond 24 hours. All negative entries and duration over 24 hours were removed and formed 'missing' values.

- Membranes ruptured to birth also had extremely long durations, with three around one year, and 25 over one week. Each of these entries were reviewed individually and any that appeared unlikely were removed.

- Duration of hospital admission was a negative value for 272 women, these were removed.

- Birthweight was below 1500g for 22 babies, so was removed, as on inspection none of these babies were admitted to SCBU, therefore their birthweight data at term is implausible.

\subsubsection{Issues arising during data collection and analysis}

Variable parameters proved to be slightly different between the three data sources. The data collection tool was created without reference to the NDHB Maternity Plus coding system, and the data from COMCORD was supplied significantly later than the primary data collection. A good example of this was postpartum blood loss, which was recorded in the primary data collection with values of under $500 \mathrm{mls}, 500-999 \mathrm{mls}$ and over $1000 \mathrm{mls}$, because those intervals are used in the Referral Guidelines (MOH, 2012) as decision points. However, blood loss is reported by NDHB and COMCORD in $250 \mathrm{ml}$ increments over $500 \mathrm{mls}$, and at NDHB the highest value is "over $750 \mathrm{mls"}$ ". To enable the reader to consider all data, it has been displayed as it is, in the Findings chapter. In simpler cases, such as description of greater diversity within mode of birth (i.e. specific instrumental methods), those figures 
Methodology

were aggregated to provide overall totals in new columns, leaving the original data intact for reference.

The COMCORD sample was useful, but due to the long process of negotiating and obtaining the data sample it wasn't truly apparent what the limitations inherent in this data were until the data were received. Detailed data is supplied to MMPO by midwives, but some data fields are not collated in the COMCORD database, and it is unclear why this occurs. An example of this are consultation codes, which are used when midwives indicate they have consulted with another professional for a maternal or neonatal condition. This meant it was not possible to identify women who had referrals for PROM or chorioamnionitis, or reasons why babies may have been admitted to SCBU. Also not reported was administration of IAP, or neonatal antibiotic treatments even those these data points are collected. The issues with data quality were reported back to the COMCORD contact person, but it was not possible to correct that data. These factors limited the usefulness of this data and on reflection, it highlights the value of prospective data collection specific to a research project. In addition to measurable outcomes for women and babies, the researcher intended to identify documentation of clinical decision-making, including informed consent processes, maternal engagement, and evidence of consultation with obstetric staff. Midwives are required to document clinical decisions and rationale including processes of informed consent (Midwifery Council of New Zealand, n.d.). However, once data collection had commenced, it became clear that the quality of documentation varied significantly, and it was difficult to know what had been discussed prior to any care decisions, particularly prior to admission to an NDHB facility. Consultation with an obstetrician prior to 24 hours postROM appeared to be well recorded for women who had presented to an NDHB facility; however clinical notes relating to events in the community were only added to a woman's NDHB file in one case. It was not possible to reliably ascertain whether women were seen by their LMC prior to admission or what consultations or decisions were made, unless the LMC wrote a history into the clinical notes or the woman informed core staff.

\subsubsection{Carrying out the clinical records review}

The clinical records review commenced with one visit to Whangarei Base Hospital to meet with maternity managers, review the proposed data collection tool, and complete the 
Methodology

practicalities required for access to workspaces and files. Data collection required 14 visits, generally one per week, to reviews the files requested. A $15^{\text {th }}$ visit was made when it became clear that specific antenatal screening data was desirable. This was obtained using Concerto ${ }^{18}$ on a computer in the Quality and Education office. Data entry was then conducted once data collection had been completed.

\subsection{Analysis}

The data to be analysed came from clinical records relating to the intrapartum care of individual women and was written in primary and secondary care settings. Much of the data collected is routinely recorded at every birth event. This evaluation did not engage in a discourse analysis of written clinical notes as it is outside the scope of this study, however some text-based data reflected the context in which births occurred. The written clinical notes showed high variability in documentation standards and styles, application of practice guidelines and individual practices. Where appropriate, contextual notes for the researcher were added as a text field at the far right of the SPSS data fields, and not included in statistical analysis, but useful in recalling detail or discussing outcomes, such as reasons for admission to SCBU.

IBM SPSS 26 was used to analyse quantitative data and a biostatistician was consulted for guidance. Keeping statistical analysis and reporting relatively simple and clear is valued in evaluation theory, as it keeps results understandable for stakeholders who may not have statistical expertise (Posavac \& Carey, 2007). Generally, percentages were sufficient to identify differences; however, probability values and odds-ratios with confidence intervals were used where appropriate for comparisons with NDHB or COMCORD data. Tables and graphs have been used for displaying findings (Ashmore et al., 2011b; Posavac \& Carey, 2007). Inductive reasoning and explanatory data analysis looked for patterns and relationships within the data available, particularly in looking for factors that may be of influence on outcomes. Coding was used to give qualitative characteristics, such as ethnicity

${ }^{18}$ A DHB intranet site which provides a variety of services including histology reporting 
Methodology

and mode of birth, numerical values in IBM SPSS 26, to be used in calculations (Babbie, 2008).

Univariate analysis (of single variables at a time), such as maternal age or ethnicity, has been displayed as distribution charts, using either percentages or frequency. Mean and median calculations, such as duration of the stages of labour was useful to then contrast the different care paths taken. Deviation calculations were used to display the distribution of results and identify outliers, if present (Babbie, 2008).

Bivariate analysis (of two variables against each other) were used to identify patterns, and to help explain the results found in the data, for example, ethnicity and maternal smoking, and these analyses are presented in the findings chapter as 'key outcomes'.

\subsection{Demonstrating reliability and validity}

Reliability in this project is based on acceptance that the data found in the clinical records was accurate and truthful (Morse \& Field, 1995), although it was limited by what data had been recorded (Ashmore et al., 2011b). Data were taken directly and verbatim from clinical notes and recorded on the data collection tool. As clinical notes are a form of legal documentation, the veracity of the notes were accepted. This data should have a high degree of inter-rater reliability, as times, participants and sentinel events (birth of baby, mode of birth) have a finite number of values, and once documented, remain as stated unless formally altered through an amending statement. There are limitations to the use of clinical records, which will be discussed in the Findings chapter, however medical record review is a common form of data collection in health, particularly in quality improvement studies.

The gathered data was checked for errors by self-auditing the data entries against the data recorded in the collection tool. The only way it would be possible to test-retest would be to apply the data collection tool to other year groups (Bryman, 2016). A pragmatic ontological perspective would argue that the results stand as representative of their sample only (Mertens \& Wilson, 2012), and that is how they are presented.

Reliability was supported by only having one person collecting and coding the data and applying consistent measurements (Ashmore et al., 2011b; Bui, 2009). Being aware of the 
Methodology

possible issues inherent in documentation can also assist in protecting the reliability of the findings (Babbie, 2008). Practitioners rely on good documentation as a record of events for themselves, other practitioners, and the women/whanau concerned, and as protection in the event of a practice or legal challenge, therefore clinical notes must be considered to be credible (Morse \& Field, 1995), and are, ultimately, the tangible record that exists (Babbie, 2008).

Data errors of a wide variety were found in 66 of the 157 files reviewed (42\%), which undermines the ability to have full confidence in the NDHB Solutions Plus data. The process at NDHB of transcribing the clinical notes to the Labour Record and online into to Solutions Plus appeared to create a surprising number of errors. This issue is discussed further in the findings and discussion chapters, and a full record of errors is available in Appendix 7 (NHI numbers were provided to NDHB but are redacted in this thesis).

Validity in this study was not overly complex, with a non-randomised sample including all women birthing over the course of one year. To promote internal validity, the data collection tool was tested on a small number of files on the first day of data collection, with a process of review and amendment before formally starting data collection (Ashmore, Ruthven, \& Hazelwood, 2011c). For example, maternal smoking was not originally a variable but was identified as a necessity. Therefore, the first files were recalled, and their data revised on a subsequent visit. No further changes to the data collection tool were made.

Face validity is the measure of whether the outcomes feel right or appear correct, and was supported by asking an expert, (in this case an NDHB Quality midwife), to review any questions that arose from the data itself (Ashmore et al., 2011b; Bryman, 2016). Externally, there were no events which could have affected outcomes, such as taking a sample from winter months only, and the data collected was from a snapshot in time which does not risk participants aging or leaving the area partway through labour. There was no scope for the Hawthorne effect, where for example, midwives or women were aware of the proposed project and altered their behaviour, as the project was not advertised and data were collected retrospectively (Ashmore et al., 2011b; Babbie, 2008; Mertens \& Wilson, 2012). Treatment fidelity included the consideration of whether care provided was as per the NDHB PROM policy (Mertens \& Wilson, 2012). 
Methodology

\subsection{Limitations of this study}

This retrospective, observational evaluation study which was based upon a clinical records review and data downloads could only access data that was recorded about specific birth events within NDHB. The data collected during the clinical notes review relies on adequate and accurate documentation in the clinical notes. The data downloads both rely on accurate transcription from the clinical notes to further paper-based and electronic records, which has been completed by multiple individuals. The source of data for this study relies completely on the work of others, and accuracy of the data downloads was seen to be vulnerable to varied errors. To increase validity in subsequent studies, it would be preferable to manage data collection prospectively which may also enable comparison of outcomes for women randomised to either a case or control group, or with women birthing at a different facility.

This study did not compare antibiotic therapy regimes though there is significant research regarding this area of management. NDHB policy is clear in recommending benzylpenicillin as the antibiotic prophylactic of choice to reduce the risk of neonatal EOGBS, and no other antibiotics were used as IAP. Amoxycillin-clavulanate was used as treatment of suspected chorioamnionitis in a limited number of cases.

The COMCORD data limited the study, by not being able to accurately identify women who experienced rupture of membranes prior to labour establishing. The data were supplied with a variable named "membranes ruptured to birth", which has been used as the next best option in comparing key outcomes, but does not differentiate between spontaneous or artificial rupture of membranes, such as during an induction of labour. Further, the sample is too large for a hand search to be practical.

Omitting home births may have excluded some women who used expectant management following PROM, however it was not feasible to obtain incidence data or clinical notes from isolated LMC midwives. Birthing women and practitioner perspectives are not investigated through interviews or surveys, which would have added depth and complexity, particularly in representing Māori perspectives and experiences. 
Methodology

Overall, this evaluation focuses well on the data retrieved for the PROM sample itself but is limited by reliance on data recorded by others. It is a valuable lesson for the beginner researcher.

\subsection{Dissemination of information}

Dissemination of findings to stakeholders forms the end phase of outcome evaluation process (Mertens \& Wilson, 2012). Following data collection, I met with the Midwifery Manager and provided her with a summary of the data I had reviewed and with a log of the errors I had found. Providing this information to her at that stage was ideal as it gave us both an opportunity to discuss progress during an otherwise lengthy process and provided her with feedback about the nature of data errors. All information provided to the Midwifery Manager was anonymous, with NHI numbers but no patient or staff names. The only way to identify individuals would be to search records herself. Another topic that was able to be discussed at that time was the low numbers of women receiving appropriate antenatal screening. This topic can be addressed through practitioner education. Under Standard Seven of the Standards of Practice, midwives are expected to use evidence-based practice and engage with research and quality improvement processes (New Zealand College of Midwives, 2015). The findings of the full project will be presented to NDHB staff and access-holders at a maternity morbidity and mortality meeting, and the thesis will be made available following assessment.

This chapter has outlined the methodology for this process and outcome evaluation, and discussed the progression through the stages of project planning and data collection. The next chapter will detail the results found from the clinical records review and statistical comparisons with wider NDHB and COMCORD data. 


\section{$4 \quad$ Findings}

This chapter presents the findings from the data analysis, with descriptive data provided for demographic, antenatal, intrapartum, birth and neonatal outcomes. Where it is appropriate to make comparisons between groupings, this has been done with independent sample ttests or odds-ratio calculations. Later in the chapter there are wider comparisons of key outcomes, based on characteristics that may be queried as being causative of issues for women and/or neonates.

The analysis compares data from three sources. The primary source of data used in analysis is the PROM sample, which comes from reviewing the clinical notes of women birthing within NDHB facilities, with PROM of $\geq 18$ hours, taken from the one-year period 1 June 2018 to 30 May 2019. The PROM sample includes 123 maternal and 124 neonatal records (one second twin is included in neonatal calculations). Analysis of this data is in-depth and provides an interesting insight into outcomes for women with PROM of over 18 hours.

The secondary source of data is a database download supplied by NDHB, of all other births at term over the same one-year period and includes 1717 births. The women and babies in the PROM sample have been removed from the NDHB sample, after identifying them using their NHI numbers. These two groups of data are therefore independent of each other.

The third source of data is a database download, supplied by the New Zealand College of Midwives Clinical Outcomes Research Database (COMCORD), and includes 28502 births at term during 2016. This was the most recent year available for analysis. The COMCORD data is included to provide a comparison with wider New Zealand outcomes where possible. This sample was taken from birth data supplied by midwives who use MMPO as their claiming agent for funding and represents about half of all New Zealand births (MMPO, 2016). The COMCORD data did not include women who had either private obstetricians or DHBemployed midwifery teams as their LMC and may therefore differ compared to a complete national dataset. However, as the NDHB area does not have any private obstetricians, COMCORD provides an adequate comparator within the scope of this study. A limitation of 
Findings

the COMCORD data is that it is taken from 2016, so is a different period from the PROM and NDHB samples.

Some women in the COMCORD data will have had PROM, however 4937 (17.3\%) of women in the COMCORD data did not have a time and date of ROM entered. Of the 2550 (8.95\%) women in the COMCORD sample who were recorded as having had membranes rupture prior to labour, 1002 women also had induction of labour, and it is unclear which event occurred first. Therefore, induction of labour would be a confounding factor which is unable to be controlled for. The COMCORD sample is present solely to serve as a comparison of national outcomes and is not the focus of this thesis. That aside, at the end of this chapter the outcomes for COMCORD women who birthed with PROM $\geq 18$ hours with no induction of labour at any time are presented to compare with the PROM sample. The results are similar, despite the PROM sample including women induced into labour subsequent to PROM.

The data in this chapter is presented where possible in combined tables, working through demographic, antenatal, intrapartum, birth outcomes, postpartum and neonatal findings. Where variables are examined with greater depth, smaller tables provide comparisons. Comparisons of key outcomes follow at the end of the chapter and provide deeper analysis of causative relationships.

\subsection{Demographic findings}

Demographic data includes maternal age, ethnicity, body mass index, parity, smoking status, and gestation at day of birth (Methods of recording ethnicity varied between the three samples, with COMCORD recording a wider variety of ethnicity than both the PROM and NDHB samples. Therefore, the COMCORD categories of Indian, Middle Eastern, Latin American, African and 'Other' have been combined as 'Other' in analysis for this project.

Table 4-1). Ethnicity, parity, and smoking status showed interesting differences between the PROM, NDHB and COMCORD samples.

The mean age of birthing women did not differ with significance between the PROM and NDHB samples $(p=0.308)$ or indeed between the three groups, though range increased with sample size from PROM (26 years), NDHB (35 years) to COMCORD (37 years). 
NDHB had a high proportion of wāhine Māori in comparison to COMCORD, however wāhine Māori featured proportionally less in the PROM sample. Of 893 NDHB wāhine Maori, 47 (5.26\%) were in the PROM sample, while of 784 European women, 62 (7.91\%) were in the PROM sample. There was only one Pasifika woman in the PROM sample and few in the NDHB sample. Correspondingly, there were more European and "Other" women in the PROM sample, although ethnic diversity was lower overall in the PROM sample and at NDHB in comparison to national figures. Methods of recording ethnicity varied between the three samples, with COMCORD recording a wider variety of ethnicity than both the PROM and NDHB samples. Therefore, the COMCORD categories of Indian, Middle Eastern, Latin American, African and 'Other' have been combined as 'Other' in analysis for this project.

Table 4-1: Demographic comparisons of samples

\begin{tabular}{|c|c|c|c|c|}
\hline Variable & $\begin{array}{l}\text { PROM } \\
(n=123)\end{array}$ & $\begin{array}{c}\text { NDHB } \\
(n=1717)\end{array}$ & $\begin{array}{l}\text { COMCORD } \\
(n=28502)\end{array}$ & $p$ value \\
\hline Maternal age (years), mean \pm SD & $28.86 \pm 5.875$ & $28.32 \pm 5.858$ & $28.59 \pm 5.72$ & 0.308 \\
\hline \multicolumn{5}{|l|}{ Ethnicity, $\mathrm{n}(\%)$} \\
\hline Māori & $47(38.2)$ & $846(49.3)$ & $5253(18.4)$ & \\
\hline European & $62(50.4)$ & $722(42.1)$ & $17460(61.3)$ & \\
\hline Pacific Peoples & $1(0.8)$ & $40(2.3)$ & $1716(6)$ & \\
\hline Asian & $5(4.1)$ & $97(5.6)$ & $3433(12)$ & \\
\hline Indian & $3(2.4)$ & & & \\
\hline Other & $5(4.1)$ & $11(0.6)$ & $638(2.2)$ & \\
\hline Body Mass Index, mean $\left(\mathrm{m}^{2} \times \mathrm{kg}\right) \pm S D$ & $27.42 \pm 6.066$ & $28.1 \pm 6.521$ & $26.639 \pm 6.150$ & 0.222 \\
\hline \multicolumn{5}{|l|}{ Parity, $n(\%)$} \\
\hline Primipara & $67(54.5)$ & $578(33.7)$ & $11565(40.6)$ & \\
\hline Multipara & $56(45.5)$ & $1139(66.3)$ & $16937(59.4)$ & \\
\hline \multicolumn{5}{|l|}{ Smoking status ( $\mathrm{n} \%)$} \\
\hline Non-smoker & $97(78.9)$ & $1281(75.8)$ & $24015(84.3)$ & \\
\hline Smoker & $26(21.1)$ & $410(24.4)$ & $3726(13.1)$ & \\
\hline Missing & $0(0)$ & $26(1.5)$ & $761(2.7)$ & \\
\hline Gestation at day of birth (days), mean \pm SD & $277.41 \pm 8.883$ & $278.47 \pm 8.772$ & $39.7 \pm 1.336^{*}$ & 0.19 \\
\hline
\end{tabular}

Mean body mass index (BMI) was lower in the PROM sample than at NDHB, but not significantly ( $p=0.222)$. The variation is likely due to small sample size, though possibly any women with extremely high BMI plus ROM prior to labour may have been managed differently and therefore were not included in the PROM sample. Parity was recorded as 
primipara ${ }^{19}$ or multipara ${ }^{20}$ due to the small sample. The PROM sample contained a higher proportion of primiparous women than the NDHB sample. This is likely to reflect a typically longer duration of latent and established labour in primiparous births, rather than a greater propensity to spontaneously rupture membranes prior to labour. The NDHB sample displayed a higher number of multiparous women than in the COMCORD sample, which reflected the higher birth rate in the NDHB population (Stats NZ, 2020).

Smoking status was recorded as either smoker or non-smoker for simplicity (Table 4-2). Fewer women in the PROM sample smoked $(21.1 \%)$ in comparison to the full NDHB sample (24.2\%). NDHB had a high rate of smoking compared to national figures and this may contribute to a high rate of small for gestational age babies, which is reviewed later in this chapter. Māori ethnicity was associated with smoking ( $p<0.001$; OR 8.64, 95\% Cl, 3.1323.84), but the wāhine Māori represented in the PROM sample were only slightly more likely to smoke than wāhine Māori in the whole NDHB sample (42.6\% vs 39.6\%, $p=0.01)$. High rates of Māori smoking were also evident in the COMCORD sample (38.7\%).

Table 4-2: Smoking status by ethnicity

\begin{tabular}{lcccccccc}
\hline & \multicolumn{2}{c}{ PROM } & & \multicolumn{2}{c}{ NDHB } & & \multicolumn{2}{c}{ COMCORD } \\
\cline { 2 - 3 } \cline { 8 - 8 } & Mãori & Non-Māori & & Mãori & Non-Māori & & Mãori & Non-Mãori \\
\hline Non-smoker & 27 & 60 & & 499 & 782 & & 3124 & 20889 \\
Smoker & 20 & 6 & & 327 & 83 & & 1972 & 1754 \\
Total & 47 & 66 & & 826 & 865 & & 5096 & 22643 \\
\% Smokers & $42.6 \%$ & $9.1 \%$ & & $39.6 \%$ & $9.6 \%$ & & $38.7 \%$ & $7.7 \%$ \\
\hline
\end{tabular}

Gestation at day of birth was calculated in days as SPSS does not calculate in weeks and days. The mean gestation for the PROM sample was 277.41 days versus 278.44 in the NDHB sample. This probably reflected only the difference of a smaller range, not a truly significant difference $(p=0.19)$. The COMCORD data provided gestation in whole weeks (days not stated), and was therefore less accurate, however the mean gestation was 39.7 weeks (277.9 days), a midpoint between the PROM and NDHB samples. It is concluded that gestational age was unlikely to be related to PROM at term.

\footnotetext{
${ }^{19}$ First birth at over 20 weeks gestation for a woman

${ }^{20}$ Second or greater birth at over 20 weeks gestation for a woman
} 


\subsection{Antenatal care}

\subsubsection{Registration with LMC by 12 weeks gestation}

Rates of registration with an LMC by 12 weeks gestation did not differ between the PROM and NDHB sample, with $51.2 \%$ versus $49.2 \%$ registered by 12 weeks. These were well below the national average given in the New Zealand Maternity Clinical Indicators of $71.9 \%$ (Ministry of Health, 2018) and below the $68.1 \%$ found in the COMCORD sample. There was no significant association found between registration after 12 weeks and ROM prior to labour commencing in the NDHB sample $(p=0.191)$.

\subsubsection{Antenatal Screening for GBS}

Antenatal screening ${ }^{21}$ data was collected from Concerto $^{22}$ for individuals in the PROM sample and reflected inconsistent care. Urinalysis in the form of a mid-stream urine sample for culture was completed at any time during pregnancy for only $39 \%$ of women. Vaginal swabs of any kind were collected for $26.8 \%$ of women, with only $2.4 \%$ given swabs appropriate for diagnosing GBS. In the absence of GBS swabs, and the low proportion of women screened via urinalysis, the GBS colonisation status during pregnancy was unknown. As universal screening is not supported in New Zealand (Darlow et al., 2015), the point is moot. There was variation in care based on ethnicity, demonstrated in Table 4-3 below. Wāhine Māori were more likely than non-Māori women to complete urinalysis and swabs.

${ }^{21}$ There is no legal directive of required antenatal screening, however recommended tests which may identify GBS include:

- Mid-stream urine sample for urinalysis, typically collected at booking and at 24-28 weeks gestation, or as needed, to screen for asymptomatic bacteriuria or infections, including GBS bacteriuria.

- Vaginal swabs at any gestation, but preferably after the first trimester, to screen for sexually transmitted diseases, may include GBS at 35-37 weeks gestation on request (Ministry of Health, 2020a).

${ }^{22}$ Concerto reports any diagnostic results produced within Northland, Waitemata, Auckland and CountiesManukau DHBs. 
Findings

Table 4-3: Antenatal Screening by Ethnicity: PROM Sample

\begin{tabular}{|c|c|c|c|c|c|c|}
\hline \multirow[b]{2}{*}{ Variable } & \multicolumn{2}{|c|}{ Maori } & \multicolumn{2}{|c|}{ European } & \multicolumn{2}{|c|}{ Other } \\
\hline & $n$ & $\%$ & $n$ & $\%$ & $n$ & \\
\hline Total & 47 & & 62 & & 14 & \\
\hline \multicolumn{7}{|l|}{ Urinalysis Screening done } \\
\hline None done in pregnancy & 22 & 46.8 & 44 & 71 & 9 & 64.3 \\
\hline Nil GBS found & 25 & 53.2 & 18 & 29 & 5 & 35.7 \\
\hline \multicolumn{7}{|l|}{ Antenatal Swabs } \\
\hline Nil swabs done in pregnancy & 29 & 61.7 & 51 & 82.3 & 10 & 71.4 \\
\hline Swabs done, but none appropriate for GBS & 16 & 34 & 10 & 16.1 & 4 & 28.6 \\
\hline Swabs for GBS negative & 0 & 0 & 1 & 1.6 & 0 & 0 \\
\hline Swabs for GBS positive & 2 & 4.3 & 0 & 0 & 0 & 0 \\
\hline
\end{tabular}

\subsubsection{Planned versus actual place of birth}

Almost all births following PROM occurred at Whangarei Base Hospital. Forty women (32.5\%) with PROM had planned to or did commence labouring at the primary centres in Kaitaia and Bay of Islands, or at home, but only eight (6.6\%) completed their labour and birth in a primary centre (see Figure 4-1) The remainder either presented to Whangarei or transferred in labour. Transfer in labour was not collected as a separate variable in the PROM sample.

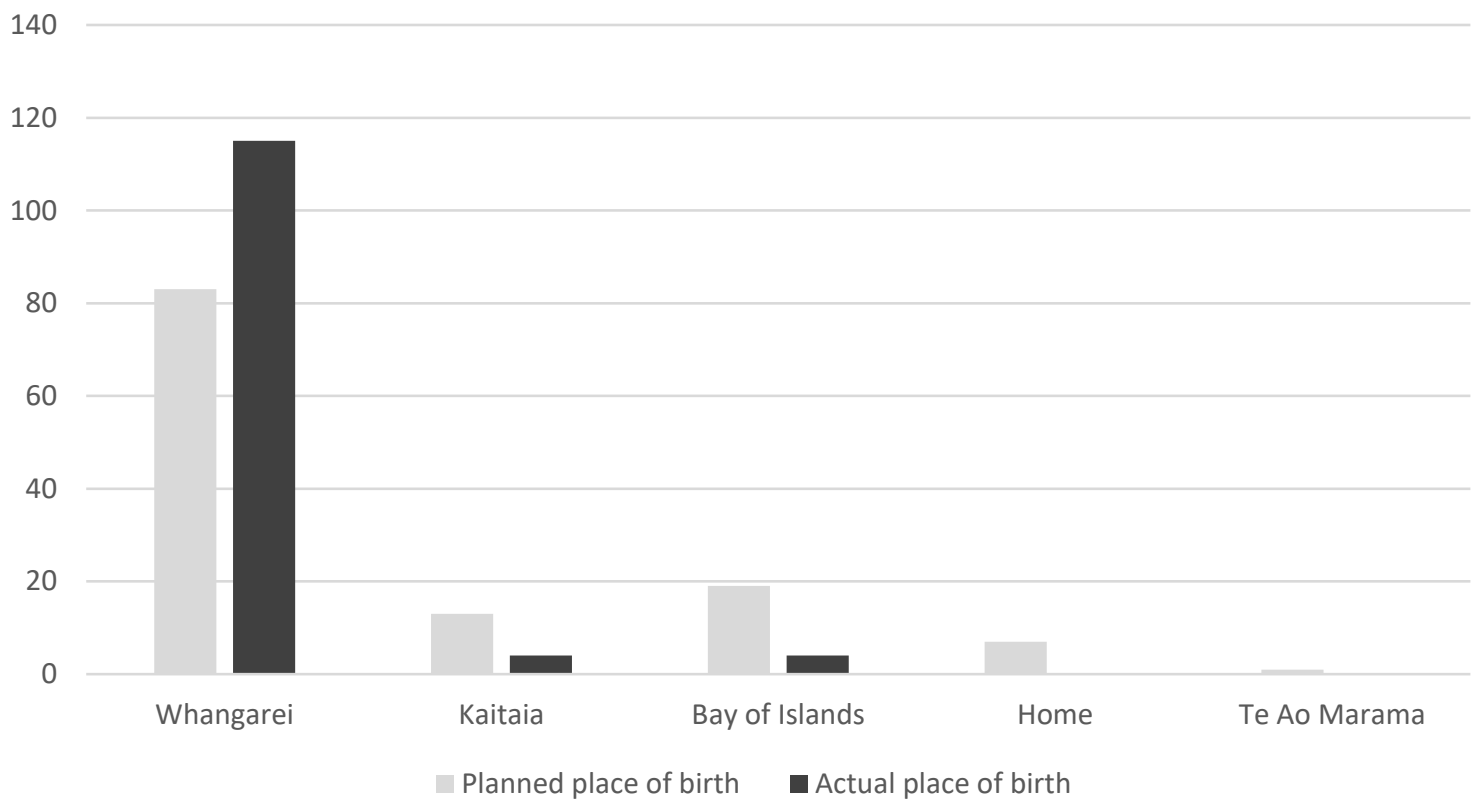

Figure 4-1 Planned place of birth versus actual place of birth (PROM sample) 
Findings

Similarly, the NDHB sample showed that while $54.2 \%$ of the NDHB sample were living within $49 \mathrm{~km}$ of Whangarei Base Hospital, the majority (81.4\%) of the NDHB sample births occurred there. Approximately half the women who lived within $49 \mathrm{~km}$ of Kaitaia and Bay of Islands Hospitals birthed in those hospitals. It is likely that distance was a significant equity factor for women who needed secondary care in labour, whether anticipated or not. Distance data was not included in the COMCORD sample.

\subsection{Intrapartum care}

Intrapartum midwifery care for women with PROM required a team approach (see Table 4-4). 
Table 4-4: Intrapartum care

\begin{tabular}{|c|c|c|c|c|}
\hline \multirow[b]{2}{*}{ Variable } & \multicolumn{2}{|c|}{ PROM } & \multicolumn{2}{|c|}{$\mathrm{NDHB}$} \\
\hline & $n$ & $\%$ & $n$ & $\%$ \\
\hline Total & 123 & 100 & 1717 & 100 \\
\hline \multicolumn{5}{|l|}{ Booked LMC } \\
\hline LMC Midwife & 113 & 91.9 & & \\
\hline Community Midwife & 6 & 4.9 & & \\
\hline Unbooked & 4 & 3.3 & & \\
\hline \multicolumn{5}{|l|}{ Labour Carer } \\
\hline LMC Midwife & 82 & 66.7 & 1503 & 87.5 \\
\hline Core Midwife & 31 & 25.2 & 209 & 12.2 \\
\hline Back up Midwife & 10 & 8.1 & & \\
\hline Missing & 0 & 0 & 5 & 0.3 \\
\hline \multicolumn{5}{|c|}{ Obstetric consultation within 24 hours } \\
\hline Yes & 65 & 52.8 & & \\
\hline No & 52 & 42.3 & & \\
\hline Birthed prior to 24 hours & 6 & 4.9 & & \\
\hline \multicolumn{5}{|c|}{ Women who sought care prior to labour } \\
\hline Yes & 73 & 59.3 & & \\
\hline No & 50 & 40.7 & & \\
\hline \multicolumn{5}{|l|}{ How was ROM diagnosed? } \\
\hline Maternal history & 122 & 99.2 & & \\
\hline Evident liquor & 66 & 53.7 & & \\
\hline Speculum & 27 & 22 & & \\
\hline Vaginal examination & 10 & 8.1 & & \\
\hline \multicolumn{5}{|l|}{ GBS status at ROM } \\
\hline unknown & 117 & 95 & & \\
\hline low risk & 3 & 2 & & \\
\hline high risk & 3 & 2 & & \\
\hline \multicolumn{5}{|c|}{ Appropriate for expectant management? } \\
\hline No & 23 & 18.7 & & \\
\hline Yes & 100 & 80.6 & & \\
\hline \multicolumn{5}{|l|}{ Expectant management used? } \\
\hline No & 10 & 8.1 & & \\
\hline Yes & 87 & 70.7 & & \\
\hline Yes, arrived in labour & 26 & 21.1 & & \\
\hline
\end{tabular}

Care was divided in the PROM sample between LMCs, their back-up midwives, and core midwives. The primary caregiver in this variable was identified as the midwife who provided either all or most intrapartum care. Care was formally handed over from LMC to core staff or secondary care in 26 labours (21.1\%) overall, while in five other cases women reported directly to Whangarei Base Hospital for induction processes and the LMC or their back-up midwife was never present during the labour and birth. NDHB reported less diversity of 
Findings

carer overall during intrapartum care, with most women cared for in labour by LMC midwives, and obstetricians were responsible for $26.1 \%$ of births. COMCORD did not identify roles or handover of care during labour.

Obstetric consultation is indicated in the Referral Guidelines (MOH, 2012), and in the NDHB PROM policy, prior to 24 hours elapsing post ROM (Bailey, 2015). Fifty-two women (42.3\%) did not receive a documented obstetric consultation within or close (within two hours) to this timeframe. Six women (4.9\%) birthed between 18-24 hours following ROM, therefore obstetric consultation was not indicated regarding ruptured membranes purposes, although five did participate in a consultation.

A possible explanation is that the women themselves may not have alerted their LMC to their ROM event, as 50 (40.6\%) had no engaged with care documented prior to labour commencing or within the first 24 hours of ROM. No documented engagement with care was associated with there being no documented obstetric consultation by 24 hours $(p<0.01)$. This statistical finding may be exaggerated however, as it is dependent on what information has been communicated via the NDHB clinical notes. It was quite possible that LMCs did provide post-ROM consultations but documented only in their own notes and not in the NDHB clinical notes. Photocopied LMC pre-labour notes relevant to ROM assessment were supplied to the NDHB file on one occasion only.

Diagnosis of ROM was by maternal history (99.2\%), visible liquor (53.7\%), speculum (22\%) and vaginal examination (8.1\%), or any combination of the above. Of the 10 women given a vaginal examination on admission, all had provided a verbal history of ROM, five had visible liquor evident on observation, and none were examined by speculum. Data as to whether ultrasound scanning or cardiotocographs were used was not collected, as neither are diagnostic of ROM (El-Messidi \& Cameron, 2010).

GBS risk status at the time of ROM was in most cases $(95.1 \%)$ recorded by the researcher in data collection as 'unknown' - the appropriate swab to detect GBS had not been done, and urinalysis earlier in pregnancy had either not been done or found no bacteriuria caused by GBS. No files noted a previous baby infected with GBS; preterm gestation at the time of ROM was present for three (2.4\%) women, and no women were reported to have a temperature of $\geq 38^{\circ} \mathrm{C}$ at time of ROM. Neither NDHB nor COMCORD collate data regarding 
GBS screening. Antenatal screening for GBS in New Zealand and NDHB will be returned to in the discussion chapter.

Decision-making as to whether expectant management was recommended for women was not always well documented, therefore each woman was flagged in data collection as eligible for expectant management if they: had ROM of under 96 hours, clear liquor, no maternal or fetal indications for IOL or birth, no digital vaginal examination, and GBS risk factors not indicative of high risk. Expectant management was assessed by the researcher as being an appropriate option for 100 (81.3\%) women in the PROM sample. Expectant management was used for $70.7 \%$ of women, plus another $21.1 \%$ arrived in labour, having used expectant management for varying periods of time prior to presentation. Any cares which may have been given to these women prior to presentation to an NDHB facility were typically not documented in clinical notes. NDHB data did not comment whether expectant management was recommended or positively used for women.

Of the women for whom expectant management was inappropriate, seven women used expectant management at home prior to any documented consultation, and when they presented in labour, they were examined and discovered to have either fetal malpresentation or fetal compromise. Ten women had a digital vaginal examination to diagnose ROM, and were therefore unsuitable for expectant management, but nine of them used it. One woman was recommended to have an actively managed labour and had ongoing consultation with obstetric staff, but declined most cares including induction of labour and IAP despite a poor clinical picture.

\subsubsection{Time factors in labour}

Within the PROM sample, 119 women established into labour ${ }^{23}$. The mean durations found in the PROM sample were all significantly prolonged in comparison to the NDHB sample -

\footnotetext{
${ }^{23}$ Established labour was recorded when stated in clinical notes, or if not stated or discrepancy existed, established labour was defined by the author as progressive contractions plus cervical dilation of $3-5 \mathrm{~cm}$ or more as per Simkin and Ancheta (2011).
} 
Findings

including that of established labour (Table 4-5). The COMCORD durations were similarly shorter than the PROM sample.

Table 4-5: Time factors in labour (hours)

\begin{tabular}{|c|c|c|c|c|c|}
\hline & \multirow[t]{2}{*}{ PROM } & \multirow[t]{2}{*}{$\mathrm{NDHB}$} & \multicolumn{2}{|c|}{ One sample T-Test } & \multirow[t]{2}{*}{ COMCORD } \\
\hline & & & $p=$ & $95 \% \mathrm{Cl}$ & \\
\hline \multicolumn{6}{|c|}{ Duration from ROM to established labour when ROM occurred first } \\
\hline$n=$ & 119 & 427 & & & \\
\hline Mean \pm SD & $39: 01 \pm 31: 47$ & $5: 53 \pm 10: 11$ & $<0.001$ & $33: 15$ to $44: 48$ & \\
\hline \multicolumn{6}{|c|}{ Duration of ROM to IOL } \\
\hline$n=$ & 34 & & & & \\
\hline Mean \pm SD & $59: 29 \pm 39: 23$ & & & & \\
\hline \multicolumn{6}{|c|}{ Duration of established labour } \\
\hline$n=$ & 119 & 1549 & & & 21914 \\
\hline Mean \pm SD & $8: 35 \pm 6: 14$ & $5: 38 \pm 5: 13$ & $<0.001$ & $7: 27$ to $9: 43$ & $5: 25 \pm 4: 27$ \\
\hline \multicolumn{6}{|c|}{ Duration from ROM to birth } \\
\hline$n=$ & 123 & 1514 & & & 23565 \\
\hline Mean \pm SD & $48: 39 \pm 33: 22$ & $4: 45 \pm 7: 47$ & $<0.001$ & $42: 42$ to $54: 37$ & $6: 18 \pm 12: 47$ \\
\hline
\end{tabular}

Duration from ROM to birth varied between wāhine Māori and non-Māori women in the PROM sample, with non-Māori more likely to birth in the first 48 hours than wāhine Māori (Figure 4-2). Beyond that point in time the absolute numbers are low, however Māori overrepresentation in the $96+$ hours group is interesting to note and comparison of labour and birth outcomes will provide useful data to assess questions of equity.

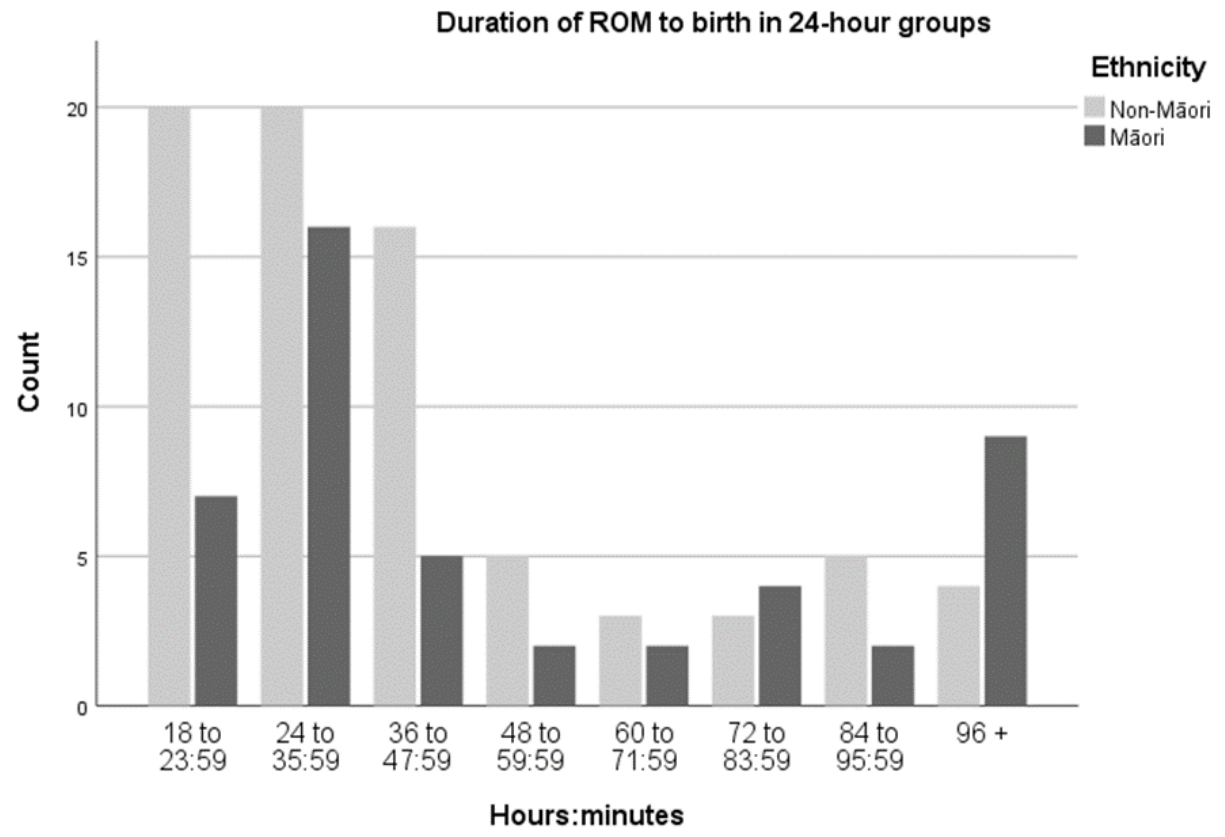

Figure 4-2: Duration of ROM to birth in 24-hour groups 


\subsubsection{Induction and augmentation of labour}

Induction of labour was not needed by $89(72.4 \%)$ of women, as 86 established into labour spontaneously, and three women had acute caesarean sections - two for breech presentation and one had planned an elective caesarean section due to her obstetric history. The remaining 34 (27.6\%) of women did have induction of labour, as contractions had not commenced during expectant management (See Table 4-6). Overall, NDHB had a slightly lower rate of induction of labour compared to COMCORD (19.3\% vs $20.7 \%$ ), however women in the PROM sample were more likely to have an induction of labour.

Of the 34 women who were induced, the mean duration of time from ROM to the start of induction was 59:26 hours (range 2:25 to 149.45 hours). This indicated that expectant management was often a component of care for many women who eventually had an induction of labour and suggests that increasing duration of time since ROM may have prompted a decision to induce labour. Maternal input into decision-making was difficult to gauge and not consistently recorded and is therefore not included in analysis.

Table 4-6: Induction and augmentation of labour

\begin{tabular}{|c|c|c|c|c|c|c|}
\hline \multirow[b]{2}{*}{ Variable } & \multicolumn{2}{|c|}{ PROM } & \multicolumn{2}{|c|}{$\mathrm{NDHB}$} & \multicolumn{2}{|c|}{ COMCORD } \\
\hline & $n$ & $\%$ & $n$ & $\%$ & $n$ & $\%$ \\
\hline Total & 123 & 100 & 1717 & 100 & 28502 & 100 \\
\hline \multicolumn{7}{|l|}{ Women offered IOL } \\
\hline No & 51 & 41.5 & & & & \\
\hline Yes & 37 & 30.1 & & & & \\
\hline Not applicable & 31 & 25.2 & & & & \\
\hline Unknown & 4 & 3.3 & & & & \\
\hline \multicolumn{7}{|c|}{ Induction of labour used } \\
\hline No & 89 & 72.4 & 1384 & 80.7 & 22602 & 79.3 \\
\hline Yes & 34 & 27.6 & 332 & 19.3 & 5900 & 20.7 \\
\hline \multicolumn{7}{|l|}{ Methods of induction } \\
\hline PGE2 Gel & 24 & 19.5 & & & 1971 & 6.9 \\
\hline ARM & 4 & 3.3 & 41 & 2.4 & 1549 & 5.4 \\
\hline Oxytocin infusion & 23 & 18.7 & 64 & 3.7 & 2327 & 8.2 \\
\hline \multicolumn{7}{|c|}{ Augmentation of labour } \\
\hline No & 53 & 43.1 & 13 & 76.5 & 22526 & 79.0 \\
\hline Yes & 70 & 56.9 & 403 & 23.5 & 5976 & 21.0 \\
\hline \multicolumn{7}{|c|}{ Methods of augmentation } \\
\hline Oxytocin infusion & 64 & 52 & 180 & 10.5 & 4309 & 15.1 \\
\hline ARM & 20 & 16.3 & 297 & 17.2 & 3692 & 13.0 \\
\hline
\end{tabular}


Methods of induction of labour included: prostaglandin gel, artificial rupture of membranes and oxytocin infusion. One method was sufficient for $52.9 \%$ of induced women, while 44.1\% needed a second method. Only one woman needed all three methods. No other methods of induction, such as misoprostol or cervical balloon, were used. Four women needed a prelabour ARM secondary to SROM.

Augmentation of labour via ARM or oxytocin infusion was used by 70 (56.9\%) of all women in the PROM sample, compared to only $23.5 \%$ of the full NDHB sample and $21 \%$ of the COMCORD sample. ARM was used as labour augmentation for 20 women (16.3\%), which was similar to the NDHB rate of $17.2 \%$, considering ROM should have already occurred. Both are higher than the COMCORD rate of $13.0 \%$. The total number of women receiving ARM for either induction or augmentation following PROM in the sample group was 24 (19.5\%). This unexpectedly high incidence of ARM occurring in women who are understood to have previously ruptured membranes is examined as a key finding later in this chapter. Oxytocin infusion was used as augmentation in 64 women (52.0\%), including 26 of the 34 women who were induced, for whom it was ongoing from the induction process. A higher rate of oxytocin use may be expected, but this was surprisingly high in comparison to that reported by both NDHB (14.8\%) and COMCORD (15.1\%).

An interesting comparison of the use of oxytocin (for either IOL or augmentation) with mode of birth exists between the data samples (Table 4-7). Oxytocin was much more commonly used for all women in the PROM sample across all modes of birth and was associated with increased numbers of instrumental and caesarean birth. Whether oxytocin caused the operative mode of birth, or the pattern of labour was already abnormal, and interventions were made was not possible to discern from the recorded data. 


\begin{tabular}{|c|c|c|c|c|}
\hline \multirow{3}{*}{\multicolumn{2}{|c|}{ Any oxytocin use }} & \multicolumn{3}{|c|}{ Mode of delivery } \\
\hline & & Vaginal & Instrumental & Caesarean \\
\hline & & \multicolumn{3}{|c|}{ Count (\%) } \\
\hline \multirow{2}{*}{ PROM } & No & $43(35.0)$ & $1(0.8)$ & $15(12.2)$ \\
\hline & Yes & $30(24.4)$ & $16(13.0)$ & $18(14.6)$ \\
\hline \multirow{2}{*}{ NDHB } & No & $1190(69.3)$ & $71(4.1)$ & $275(16.0)$ \\
\hline & Yes & $89(5.2)$ & $37(2.2)$ & 55 (3.2) \\
\hline \multirow{2}{*}{ COMCORD } & No & $17321(60.8)$ & $1541(5.4)$ & $5323(18.7)$ \\
\hline & Yes & $2208(7.7)$ & $912(3.2)$ & $1187(4.2)$ \\
\hline
\end{tabular}

\subsubsection{Maternal and fetal condition in labour}

Highest recorded maternal temperature ranged from $35.3^{\circ}$ to $39.1^{\circ}$ centigrade, with a mean of $37.1^{\circ}$ and median of $37.0^{\circ}$ (Table $4-8$ ). Pyrexia was associated with suspected chorioamnionitis $(p=0.01)$, but there were other factors attributed as the cause in some clinical notes, such as obstructed labour and the use of epidural. Three women did not have their temperature recorded. NDHB and COMCORD did not collate data about maternal temperatures.

All women in this sample were eligible for intrapartum antibiotic prophylaxis (IAP) during labour as 18 hours had elapsed following ROM, but only 102 (82.9\%) of women were given IAP. Of the 21 women who did not receive IAP, four birthed within a further two hours; one had an established labour of 20 minutes, and two women did not establish into labour prior to a caesarean. Three women $(2.4 \%)$ declined IAP, one of whom was later given amoxycillin-clavulanate for suspected chorioamnionitis. The reasons for the remaining 11 women not receiving IAP were not documented. Women not given IAP for any reason were more likely to develop clinical symptoms of chorioamnionitis than those who were given IAP (22.2\% vs $5.9 \%, p=0.05)$. GBS was not found in any blood cultures or swabs that may have been collected from these women. Neither NDHB or COMCORD included IAP or antibiotic treatment in their databases. 


\begin{tabular}{|c|c|c|c|c|}
\hline & \multicolumn{2}{|c|}{ PROM } & \multirow[t]{2}{*}{ COMCORD } & \\
\hline Variable & $n(\%)$ & $\begin{array}{l}\text { Amoxi-clav } \\
\text { given } n(\%)\end{array}$ & & $\begin{array}{c}\text { Correlation to } \\
\text { chorioamnionitis } \\
\quad p \text { value }\end{array}$ \\
\hline Total & 123 & 4 & & \\
\hline Maternal Temperature in Labour, mean \pm SD & $37.1 \pm 0.686$ & & & \\
\hline Maternal temperature $\geq 38^{\circ} \mathrm{C}$ & $11(8.9)$ & & & $<0.01$ \\
\hline \multicolumn{5}{|l|}{ Intrapartum Antibiotic Prophylaxis Use } \\
\hline Yes & $102(82.9)$ & $1 / 102(1.0)$ & & \\
\hline No & $18(14.6)$ & $2 / 18(11.1)$ & & \\
\hline Declined & $3(2.4)$ & 1/3 (33.3) & & \\
\hline Duration from IAP to birth, mean \pm SD & $8: 02 \pm 8: 34$ & & & \\
\hline \multicolumn{5}{|l|}{ Suspected Chorioamnionitis } \\
\hline No & $113(91.9)$ & & & \\
\hline Yes & $10(8.1)$ & $4 / 10(40)$ & & \\
\hline \multicolumn{5}{|l|}{ Chorioamnionitis by IAP use } \\
\hline Chorioamnionitis/IAP not used & $4 / 18(22.2)$ & & & $<0.05$ \\
\hline Chorioamnionitis/IAP used & $6 / 102(5.9)$ & & & \\
\hline Digital vaginal examinations, mean \pm SD & $4.13 \pm 2.439$ & & & $<0.05$ \\
\hline Duration from ROM to first $V E$, mean $\pm S D$ & $35: 47 \pm 31: 12$ & & & \\
\hline Duration from first $V E$ to birth, mean $\pm S D$ & $12: 51 \pm 12: 56$ & & & \\
\hline \multicolumn{5}{|l|}{ Liquor quality } \\
\hline Clear & $96(78)$ & & $22016(77.2)$ & \\
\hline Meconium & $17(13.8)$ & & $4270(15)$ & \\
\hline Blood-stained & $10(8.1)$ & & 824 (2.9) & \\
\hline Not stated & $0(0)$ & & $1392(4.9)$ & \\
\hline Total & $123(100)$ & & $28502(100)$ & \\
\hline
\end{tabular}

Chorioamnionitis was recorded in clinical notes as being clinically suspected in 10 women, seven of whom had temperatures of $38^{\circ}$ or more, although four other women also had temperatures of $38^{\circ}$ or more, with no clinical comment about chorioamnionitis. Four women (3.3\%) were given amoxycillin-clavulanate due to suspected chorioamnionitis, of which one had received a single dose of IAP, two had not received IAP and one had declined it. Incidence of chorioamnionitis was not reported by NDHB or COMCORD.

The women with documented suspected chorioamnionitis (Table 4-9) had a high rate of non-normative birth events and outcomes. This group typically had a high number of vaginal examinations, with six receiving five or more examinations between ROM and birth. This may have contributed to the high incidence of pyrexia and suspected chorioamnionitis. Four (40\%) birthed by caesarean and three (30\%) with instrumental assistance, and only three (30\%) had vaginal births. The incidence of postpartum haemorrhage of $500-999 \mathrm{mls}$ 
Findings

was $50 \%$ in this group, and four (40\%) neonates were admitted to SCBU for respiratory support of four or more hours.

Table 4-9: Suspected chorioamnionitis (PROM sample)

\begin{tabular}{|c|c|c|c|c|c|c|c|c|}
\hline Woman & IAP given & $\begin{array}{l}\text { IAP doses } \\
\text { given }\end{array}$ & $\begin{array}{l}\text { Augmentin } \\
\text { given }\end{array}$ & $\begin{array}{l}\text { VE in } \\
\text { labour }\end{array}$ & $\begin{array}{l}\text { Mode of } \\
\text { birth }\end{array}$ & PPH & Endometritis & $\begin{array}{l}\text { Neonate to SCBU } \\
\text { for resp. support }\end{array}$ \\
\hline 1 & Declined & 0 & Yes & 3 & Caesarean & $500-999 \mathrm{mls}$ & No & No \\
\hline 2 & Yes & 1 & Yes & 5 & Caesarean & $500-999 \mathrm{mls}$ & Suspected & No \\
\hline 3 & No & 0 & Yes & 9 & Instrumental & No & No & Yes \\
\hline 4 & No & 0 & Yes & 7 & Instrumental & No & No & No \\
\hline 5 & Declined & 0 & No & 3 & Caesarean & $500-999 \mathrm{mls}$ & No & Yes \\
\hline 6 & Yes & 1 & No & 9 & Vaginal & No & No & Yes, other reasons \\
\hline 7 & Yes & 1 & No & 1 & Vaginal & No & No & No \\
\hline 8 & Yes & 3 & No & 6 & Caesarean & $500-999 \mathrm{mls}$ & No & No \\
\hline 9 & Yes & 3 & No & 4 & Vaginal & No & No & Yes \\
\hline 10 & Yes & 4 & No & 13 & Instrumental & $500-999 \mathrm{mls}$ & No & No \\
\hline
\end{tabular}

Digital vaginal examinations ranged in the PROM sample from nil to 13 . The mean number of vaginal examinations was 4.13 , with a median of 4 . Increasing numbers of vaginal examinations were associated with increased risk of suspected chorioamnionitis $(p<0.05)$ see (Figure 4-3).

Five women did not receive a vaginal examination; of which two had acute caesarean sections - one for known breech presentation and one had planned an elective caesarean section. The other three women delivered spontaneously prior to any vaginal examination. Only four multiparous women received more than five vaginal examinations. NDHB and COMCORD did not report on timing or numbers of vaginal examinations. 


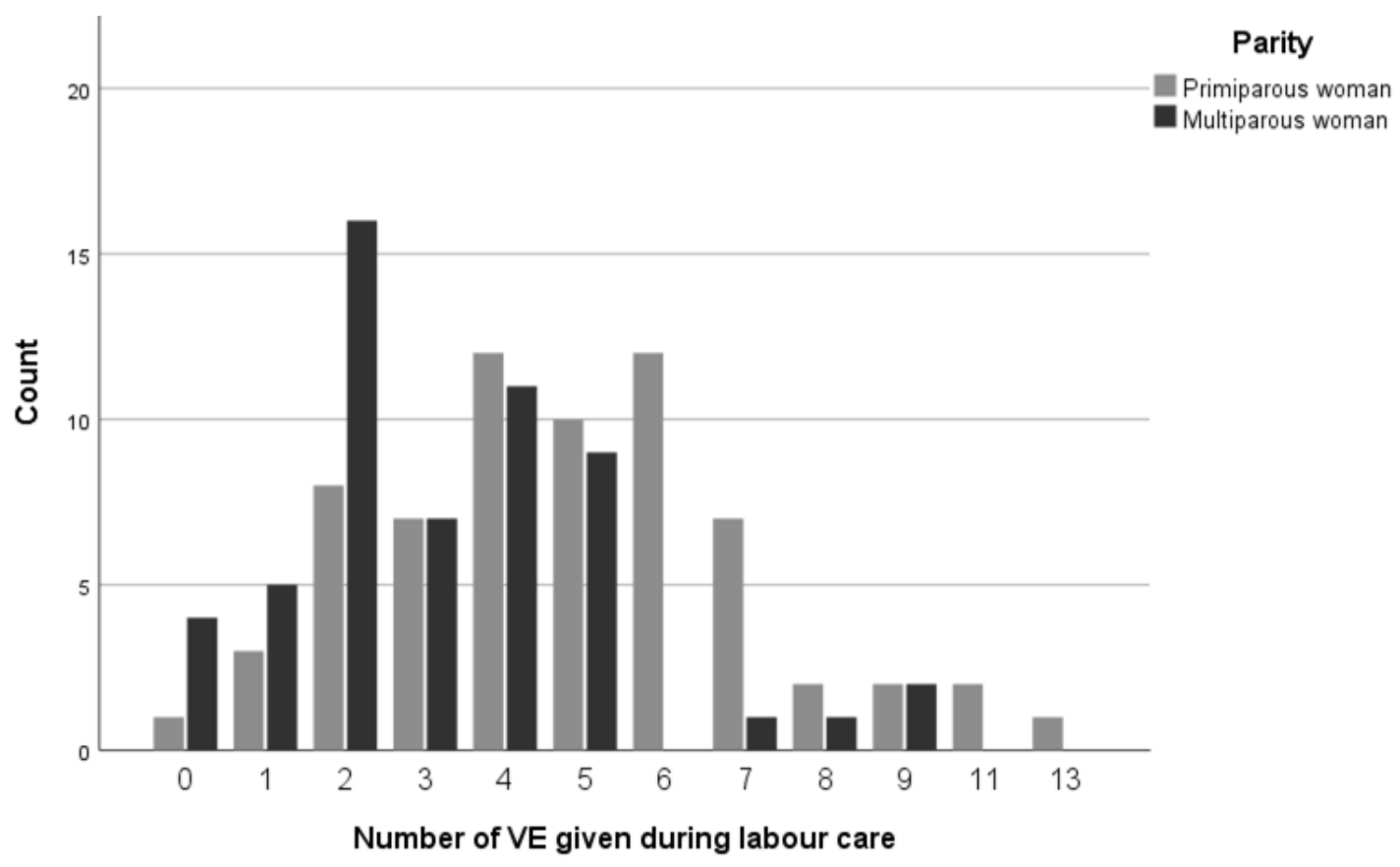

Figure 4-3: Clustered bar count of number of vaginal examinations given during labour care, by parity

Vaginal examinations commonly occurred prior to women being given IAP. This was seen in 79 women, representing $78.2 \%$ of all women who had both IAP and a vaginal examination. One woman had a duration of time from vaginal examination to IAP of over four days. This was an outlier and has been removed to calculate the mean. The mean duration from first vaginal examination to IAP in these remaining 78 women was 6:00 hours, with a median of three hours.

Liquor was clear in 96 (78\%) of labours, blood-stained in $10(8.1 \%)$ and meconium liquor was found in 17 (13.8\%) of labours. Of the 17 labours with meconium liquor, five had maternal temperatures of $37.5-37.9^{\circ}$, and three had maternal temperatures of $38-38.9^{\circ}$ with suspected chorioamnionitis. Primiparous women were more likely to have meconium liquor than multiparous women $(22.4 \%$ vs $3.6 \%, p<0.05)$. NDHB did not collate statistics regarding liquor quality, so comparison is not possible; there was also little distinction found in clinical notes between thick/fresh and thin/old meconium liquor. COMCORD provided useful data, reporting a similar rate of clear liquor (77.2\%), meconium liquor (15\%) but less bloodstained liquor (2.9\%). 


\subsubsection{Pain relief used}

Pain relief was used by 116 (94.3\%) of women in the sample (Table 4-10). The pain relief use reported in this section may reflect women using more than one form of pain relief, therefore the percentages do not add up to $100 \%$.

The most common complementary forms of pain relief were water (32.5\%), massage (21.1\%) and heat (21.1\%). Waterbirth was used by 5 (4.9\%) of women, with generally good outcomes for mothers or babies. COMCORD reported on waterbirths (9.8\%) but not on water used in labour. Pharmacological pain relief included entonox (65.9\%), morphine (30.9\%), epidural (35.8\%) and spinal anaesthesia (13.8\%). The rate of morphine use is of interest even though it is not possible to compare it to the NDHB or COMCORD samples. Morphine was introduced in 2014 as an alternative to pethidine, which was reported as used by $2.2 \%$ of the COMCORD sample. Morphine does not appear in the NDHB data as there was no coding field for it at the time of data collection, and it is suspected the relative absence in the COMCORD sample is for a similar reason.

Epidural was used more than twice as commonly in the PROM sample (35.8\%) compared to NDHB (14.8\%) and COMCORD (17.8\%) data. NDHB only report pain relief data for epidural and 'anaesthesia' (18.9\%), which appears to include all the epidurals (i.e. double reporting), and probably includes general and spinal anaesthesia. In comparison to the PROM sample, COMCORD reports lower entonox use, lower epidural use, but higher spinal and general anaesthesia, possibly reflecting a higher incidence of elective caesarean births in other DHB areas. 
Findings

Table 4-10: Pain relief used

\begin{tabular}{|c|c|c|c|c|c|c|}
\hline & \multicolumn{2}{|c|}{ PROM } & \multicolumn{2}{|c|}{ NDHB } & \multicolumn{2}{|c|}{ COMCORD } \\
\hline & $n$ & $\%$ & $n$ & $\%$ & $n$ & $\%$ \\
\hline Nil & 7 & 5.7 & & & 15907 & 55.8 \\
\hline Water & 40 & 32.5 & & & $2793 *$ & 9.8 \\
\hline TENS & 2 & 1.6 & & & 225 & 0.8 \\
\hline Entonox & 81 & 65.9 & & & 12119 & 42.5 \\
\hline Massage & 26 & 21.1 & & & & \\
\hline Heat & 26 & 21.1 & & & & \\
\hline Acupressure & 2 & 1.6 & & & & \\
\hline Coffee & 1 & 0.8 & & & & \\
\hline Sterile water injections & 2 & 1.6 & & & & \\
\hline Homeopathy & 3 & 2.4 & & & & \\
\hline Pudendal block & 1 & 0.8 & & & 247 & 0.9 \\
\hline Pethidine & 1 & 0.8 & & & 624 & 2.2 \\
\hline Fentanyl PCA & & & & & 87 & 0.3 \\
\hline Morphine & 38 & 30.9 & & & & \\
\hline Epidural & 44 & 35.8 & 254.0 & 14.8 & 5085 & 17.8 \\
\hline Spinal & 18 & 13.8 & & & 4260 & 14.9 \\
\hline Combined epidural/spinal & & & & & 327 & 1.1 \\
\hline General anaesthetic & 1 & 0.8 & 324.0 & 18.9 & 414 & 1.4 \\
\hline Other & & & & & 223 & 0.8 \\
\hline Not known & & & & & 14 & 0.05 \\
\hline
\end{tabular}

*this number reflects waterbirths, not water used in labour

Less common forms of pain relief included TENS (1.6\%), acupressure (1.6\%), homeopathy $(2.4 \%)$, pudendal block $(0.8 \%)$ and pethidine $(0.8 \%)$, which has been phased out of practice following the introduction of morphine. An unexpected form of 'pain relief' was coffee, which was also documented in the clinical notes as intended to help induce and augment labour. The benefit of coffee for either purpose was not apparent in the clinical notes, and evidence for this practice was not identified.

\subsection{Birth outcomes}

Mode of birth was affected by women having a history of PROM. Spontaneous vaginal birth was less common, while instrumental and caesarean births were more common in the PROM group (Table 4-11).

Reasons for caesarean birth were most typically failure to progress in first stage $(21.2 \%)$ or second stage (21.2\%), followed by fetal distress (15.2\%) and malpresentation or malposition (15.2\%). Obstructed labour was stated as the cause for $9.1 \%$ of caesarean births, with the 
Findings

remainder either 'other' or not stated. There were six (4.9\%) waterbirths in the PROM sample versus COMCORD (9.8\%), however the NDHB data does not include waterbirths. The number of waterbirths in the COMCORD data reflects that data was taken from midwifery-led practice.

Table 4-11: Birth outcomes

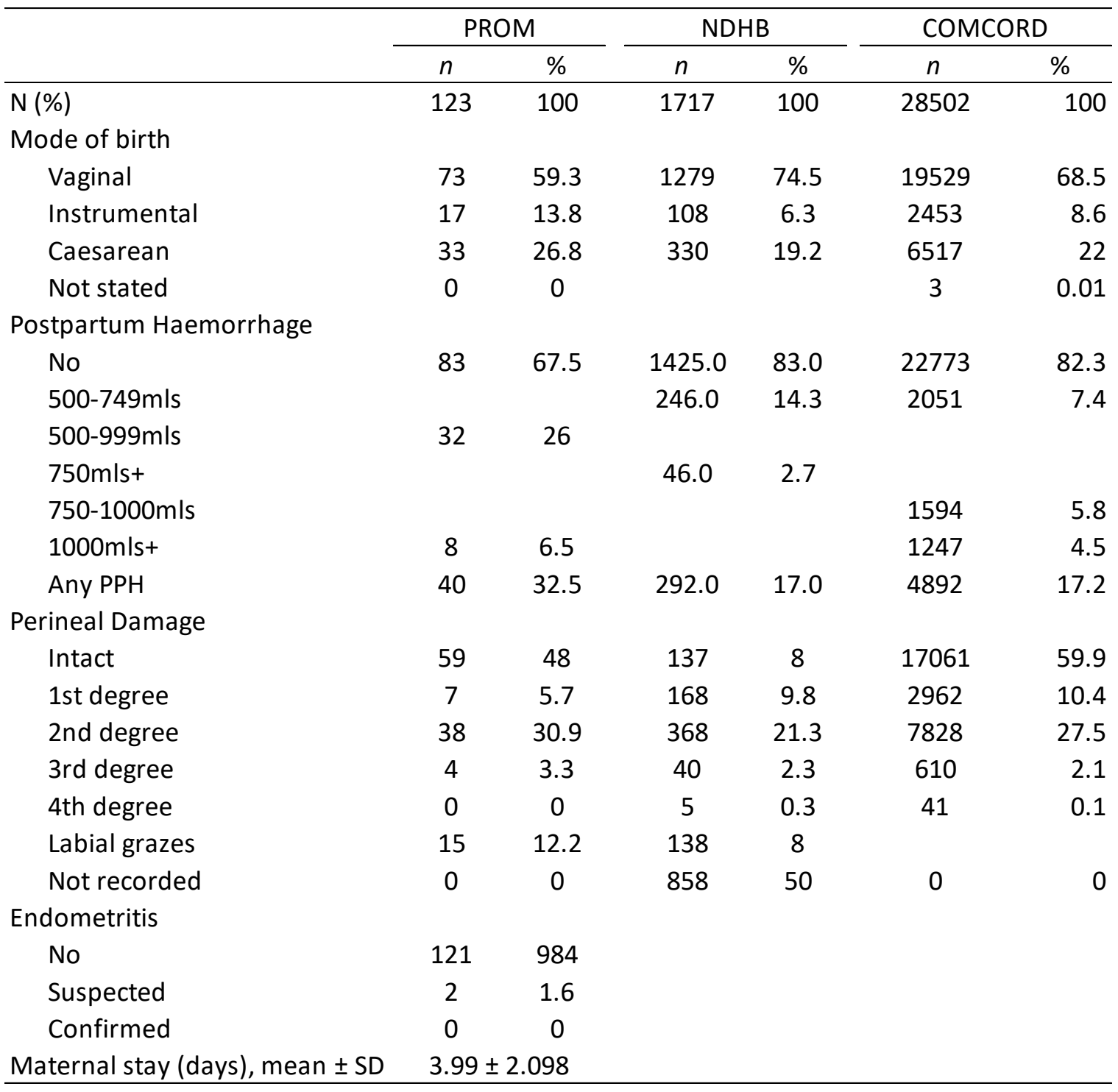

Postpartum blood-loss was recorded with differing parameters across the three samples and is presented in the table as it has been recorded. The PROM sample recorded values within parameters which are referral points under the Referral Guidelines (Ministry of Health, 2012). NDHB records postpartum haemorrhage ${ }^{24}$ only as a measure of over $500 \mathrm{mls}$ 
and over $750 \mathrm{mls}$, with no entry for any other values. COMCORD recorded loss in $10 \mathrm{ml}$ increments. For analysis, the COMCORD data has been sorted into blood loss of nil postpartum haemorrhage, $500-749 \mathrm{mls}, 750-999 \mathrm{mls}$ and $1000 \mathrm{mls}+$

The incidence of any postpartum haemorrhage in women experiencing PROM was almost double that of the NDHB and COMCORD samples, with $26 \%$ of PROM sample women losing $500-999 \mathrm{mls}$ of blood. Severe postpartum haemorrhage of $\geq 1000 \mathrm{mls}$ occurred for $6.5 \%$ of women with PROM, which was higher than the NDHB incidence of postpartum haemorrhage of $>750 \mathrm{mls}$ of $2.7 \%$ and COMCORD of $\geq 1000 \mathrm{mls}$ of $4.5 \%$.

Perineal trauma data was collected for all women in the PROM and COMCORD samples, however only recorded for 859 (50\%) women in the NDHB sample which creates an issue of validity for that data. The PROM sample was closer to the COMCORD sample and reflected a slightly higher incidence of perineal trauma overall. It is possible the COMCORD data 'intact' category included labial grazes.

Episiotomy was performed on $4.9 \%$ of women in the PROM sample and $5.8 \%$ of the NDHB sample, compared to $10 \%$ of women in the COMCORD dataset. The data does not provide reasons for the lower rates in the PROM sample and NDHB, however parity and practitioner preferences may account for them.

Endometritis was clinically documented as suspected in two (1.6\%) women, both of whom had received IAP for over four hours prior to birth. One had eight vaginal examinations, a postnatal diagnosis of retained products of conception, and her baby had suspected sepsis with a diagnosis of pneumonia. The other woman had five vaginal examinations, suspected chorioamnionitis, and a caesarean section for obstructed labour. The number of vaginal examinations was not associated with the incidence of endometritis ( $p=0.167)$, though the small sample number and low incidence of endometritis in general reduces the ability to demonstrate an association. Suspected endometritis was treated with oral antibiotics in both cases, and blood cultures were negative in both cases. NDHB and COMCORD did not provide data on the incidence of endometritis.

Maternal stay from continuous admission to discharge had a mean of 3.99 days, and a median of 4 . The maximum stay was 12 days and the minimum of 1 . The upper values 
Findings

reflect the number of women admitted for observations or induction processes antenatally and greater acuity throughout the intrapartum and postnatal stay. There was also a high rate of neonatal admissions to SCBU (discussed in the next section), and the mothers of those babies tended to remain admitted to Maternity Services throughout the duration of the neonatal stay in SCBU. NDHB and COMCORD did not provide data relating to duration from admission to discharge.

\subsection{Neonatal outcomes}

The inclusion of a second twin in the neonatal analysis brings the number of babies in the PROM sample to 124. NDHB reports on numbers born alive, gender, birthweight, admission to SCBU, and breastfeeding on discharge, while COMCORD also included APGAR scores and resuscitation data (Table 4-12). 
Table 4-12: Neonatal outcomes

\begin{tabular}{|c|c|c|c|}
\hline & PROM & NDHB & COMCORD \\
\hline \multicolumn{4}{|l|}{ Variable } \\
\hline$n(\%)$ & $124(100)$ & $1717(100)$ & $28480(100)$ \\
\hline Neonatal weight $(\mathrm{g})$, mean \pm SD & $3507.8 \pm 510.9$ & $3497.9 \pm 516.1$ & $3523.5 \pm 499.3$ \\
\hline Neonatal birthweight centiles & $45.788 \pm 29.26$ & $43.37 \pm 29.81$ & \\
\hline Māori birthweight centiles & $44.136 \pm 30.181$ & $41.990 \pm 30.035$ & \\
\hline Non-Māori birthweight centiles & $47.362 \pm 28.6$ & $44.707 \pm 29.562$ & \\
\hline Smoker birthweight centiles & $38.712 \pm 27.309$ & $34.238 \pm 29.118$ & \\
\hline Non-smoker birthweight centiles & $48.118 \pm 29.42$ & $46.34 \pm 29.349$ & \\
\hline \multicolumn{4}{|l|}{ APGAR, mean \pm SD } \\
\hline $1 \mathrm{~min}$ & $8.42 \pm 1.702$ & & $8.5 \pm 1.385$ \\
\hline 5 mins & $9.38 \pm 1.22$ & & $9.52 \pm 0.92$ \\
\hline 10 mins & $9.63 \pm 0.959$ & & $9.76 \pm 0.779$ \\
\hline \multicolumn{4}{|l|}{ Neonatal gender, $n$ (\%) } \\
\hline Female & $61(49.2)$ & $838(48.8)$ & $13972(49.0)$ \\
\hline Male & $63(50.8)$ & $878(51.1)$ & $14523(51.0)$ \\
\hline Unassigned & $0(0)$ & $1(0.6)$ & $7(0.02)$ \\
\hline \multicolumn{4}{|l|}{ Resuscitation at birth, $\mathrm{n}(\%)$} \\
\hline No & $103(83.1)$ & & $24841(87.2)$ \\
\hline Yes & $21(16.9)$ & & $3661(12.8)$ \\
\hline IAP used/resuscitated & $15 / 102(14.7)$ & & \\
\hline IAP not used/resuscitated & $6 / 21(28.6)$ & & \\
\hline \multicolumn{4}{|l|}{ Admission to SCBU, $\mathrm{n}(\%)$} \\
\hline Not admitted & $105(84.7)$ & $1557(90.7)$ & $27382(96.1)$ \\
\hline Admitted for respiratory support $>4$ hours & $15(12.1)$ & & \\
\hline Admitted other reasons & $4(3.2)$ & & \\
\hline Admitted (reason not stated) & $0(0)$ & $159(9.3)$ & $1120(3.9)$ \\
\hline Māori admission to SCBU (any reason) & $12 / 47(25.5)$ & & \\
\hline Non-Māori admission to SCBU (any reason) & $7 / 76(9.2)$ & & \\
\hline \multicolumn{4}{|l|}{ Neonate treated with IV antibiotics, n (\%) } \\
\hline No & $110(88.7)$ & & \\
\hline Yes & $14(11.3)$ & & \\
\hline \multicolumn{4}{|l|}{ Respiratory diagnosis, n (\%) } \\
\hline Nil issues & $111(89.5)$ & & \\
\hline Respiratory distress syndrome & $5(4.0)$ & & \\
\hline Transient tachypnoea of the newborn & $3(2.4)$ & & \\
\hline Other & $5(4.0)$ & & \\
\hline \multicolumn{4}{|l|}{ Neonatal mortality, n (\%) } \\
\hline No & $123(99.2)$ & 1713 (99.8) & $28450(99.8)$ \\
\hline Stillbirth & $0(0)$ & $3(0.17)$ & $42(0.15)$ \\
\hline Neonatal death & $1(0.8)$ & $1(0.6)$ & $10(0.04)$ \\
\hline Neonatal stay, mean \pm SD & $3.1 \pm 2.036$ & & \\
\hline \multicolumn{4}{|l|}{ Breastfeeding status on discharge*, n (\%) } \\
\hline Exclusive & $107(86.3)$ & $1554(90.5)$ & $19750(69.3)$ \\
\hline Fully & $0(0)$ & $47(2.7)$ & $2138(7.5)$ \\
\hline Partial & $13(10.5)$ & $29(1.7)$ & $3774(13.2)$ \\
\hline Artificial & $3(2.4)$ & 49 (2.9) & $1994(7.0)$ \\
\hline Missing & $1(0.8)$ & $38(2.2)$ & $846(3.0)$ \\
\hline
\end{tabular}

**NZCOM data represents breastfeeding status at 2 weeks postpartum 
The mean neonatal weight was $3507.8 \mathrm{~g}$ (range $2345-4945 \mathrm{~g}$ ), a non-significant $10 \mathrm{~g}$ difference from the NDHB mean of 3497.98g $(p=0.831)$, and 15.7g different to the COMCORD mean. The median weights were also close, though the larger pool of subjects in the NDHB and COMCORD samples produced wider ranges. Birthweights in the PROM sample appeared to influence the duration of ROM to birth (see Figure 4-4), with lower birthweights associated with longer durations $(p=0.01)$, though the aetiology of this is unclear, and was not seen in a comparative scatter-gram for the NDHB sample (see \& Figure 4-5).

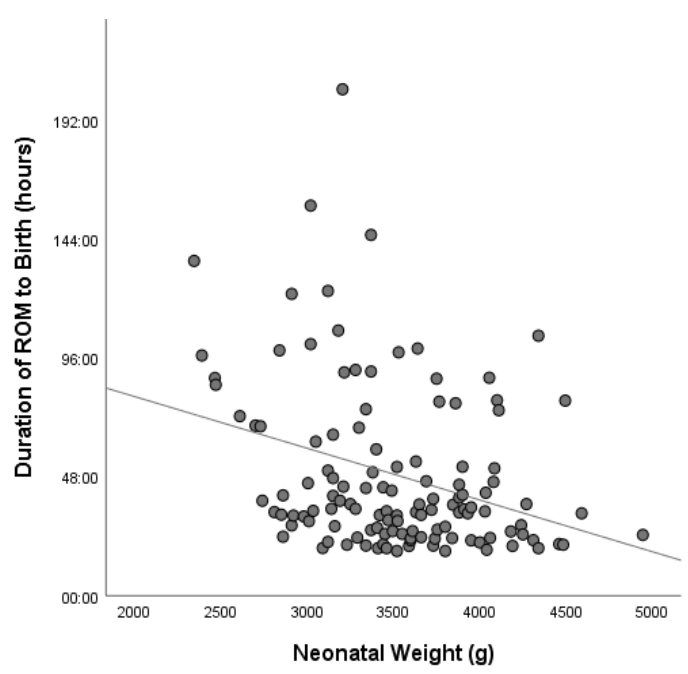

Figure 4-4 PROM sample duration of ROM to birth (hours) by neonatal weight (g)

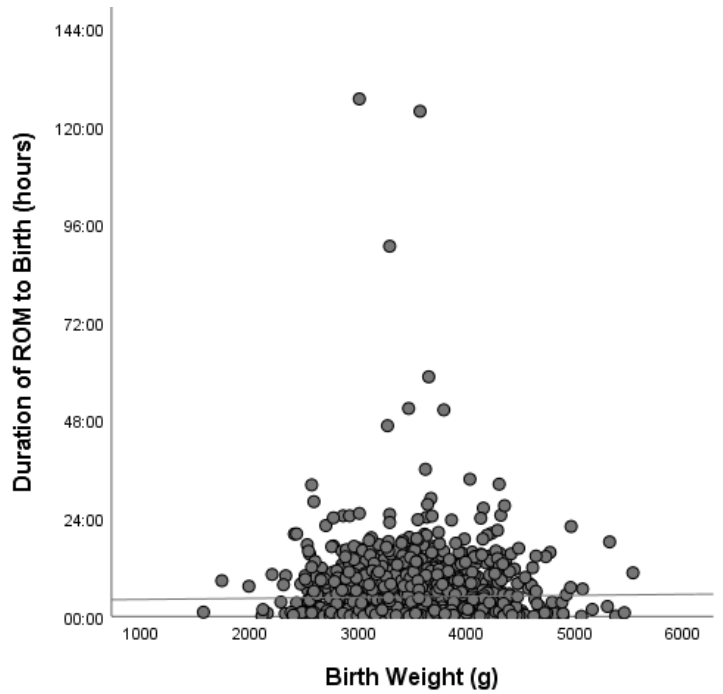

Figure 4-5: NDHB duration of ROM to birth (hours) by neonatal weight

Birthweight centiles for both the PROM and NDHB samples were calculated using the Gestation Network (n.d.) customised centile calculator and the height and weight stored for women by NDHB. Centiles were not stated in or calculated for the COMCORD sample as maternal height and weight data was not included. At the time of data collection NDHB did not routinely use or record neonatal weight centiles, and NDHB recording of ethnicity does not match the Gestation Net ethnicity groups. For the purposes of this calculation all Pacific Peoples have been identified as "other Pacific Islander", and all Asians have been identified as "Other Asian".

The histograms in the figures below provide a comparison between birthweight centiles in the PROM and NDHB samples. Neither the PROM sample (Figure 4-6) or the NDHB sample 
Findings

(Figure 4-7) fits well under a normal curve, with skewness of 0.331 and 0.3 respectively, with atypically high numbers of small and large for gestational age babies.

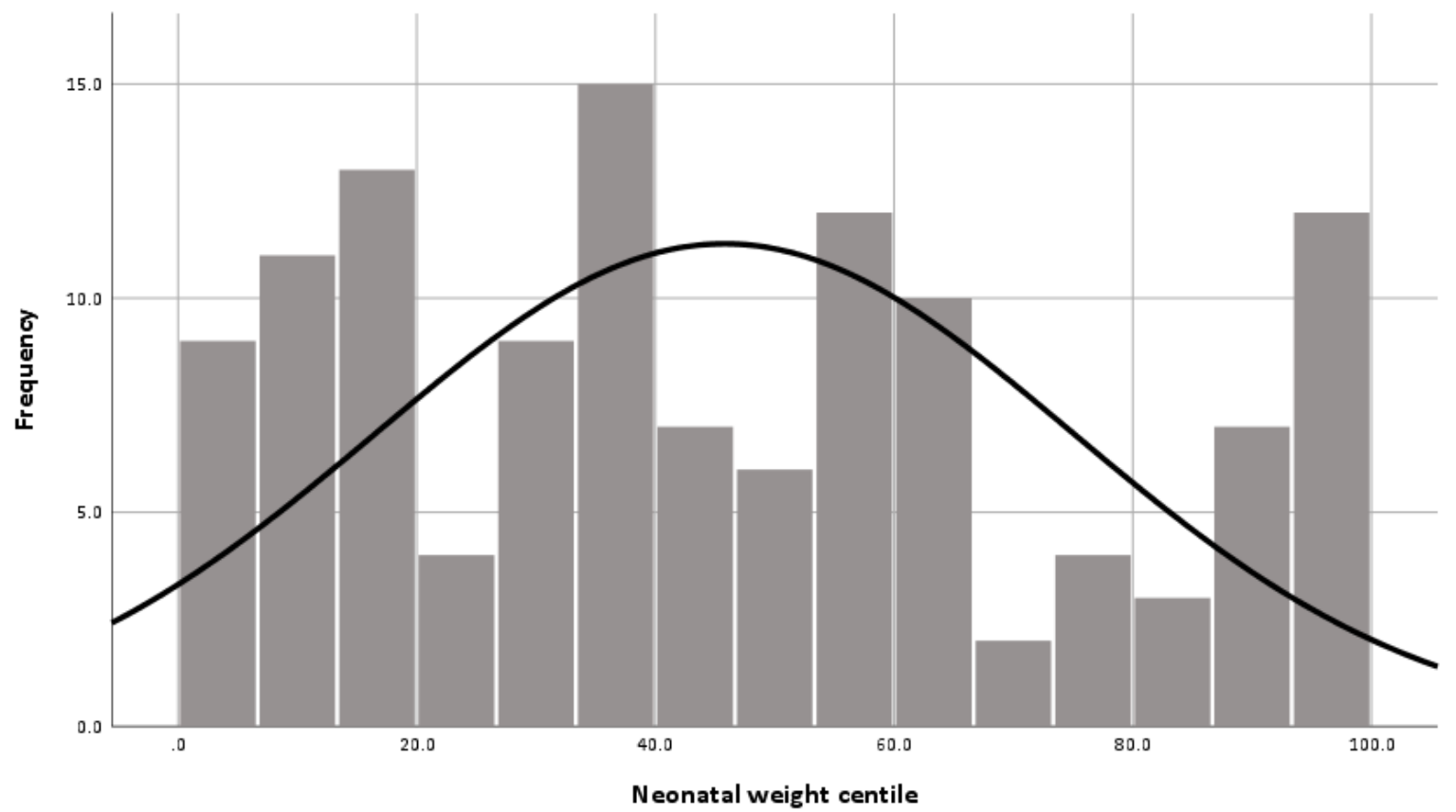

Figure 4-6: Histogram of PROM sample birthweight centiles

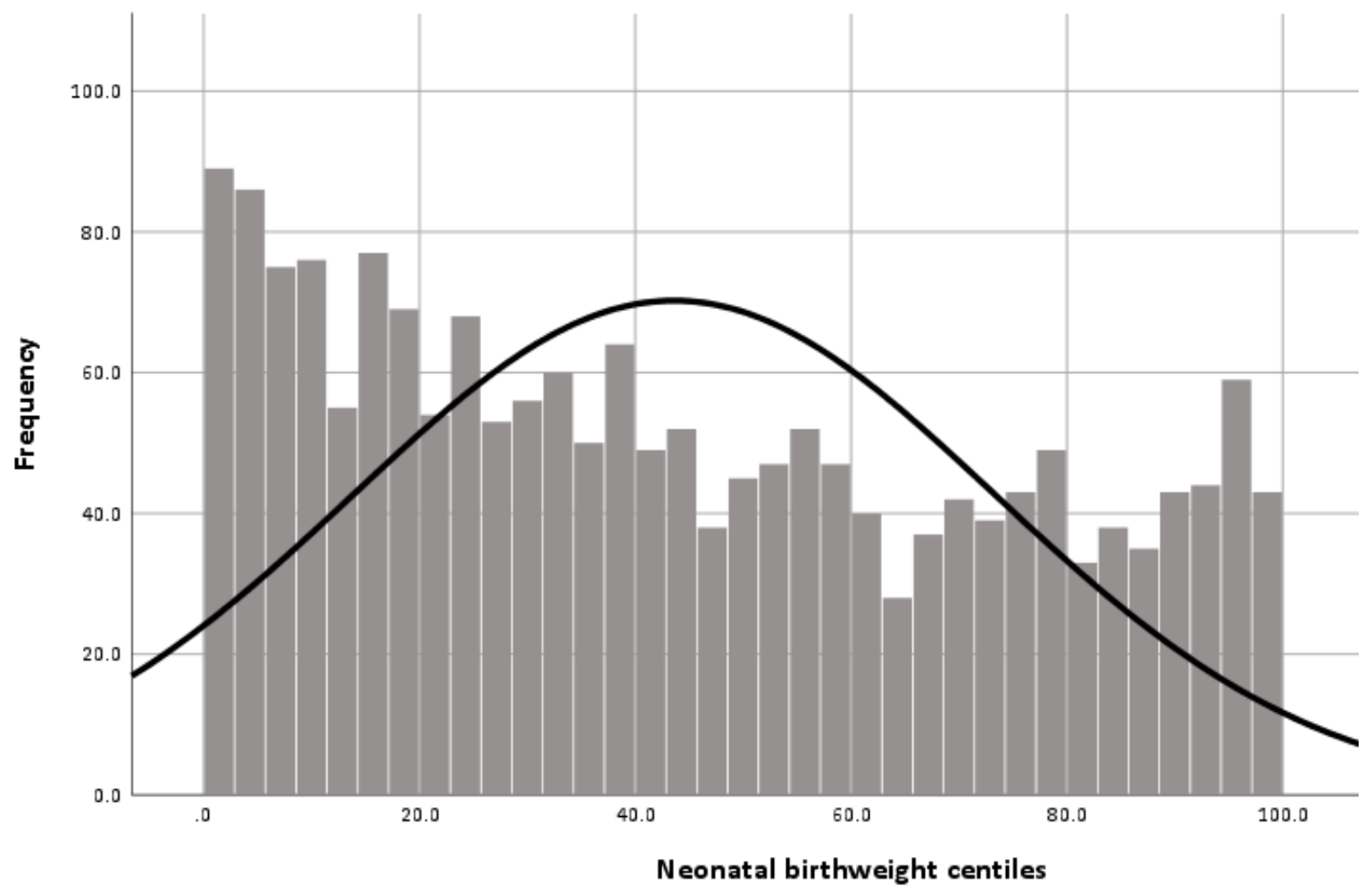

Figure 4-7: Histogram of NDHB birthweight centiles 
APGAR scores were documented for all babies in the PROM sample, with $75 \%$ of babies having a score of either 9 or 10 at one minute, increasing to $88.7 \%$ at five minutes. NDHB does not collate APGAR scores for statistical purposes, though they are recorded in individual files. COMCORD does collate APGAR scores and report slightly higher mean scores with wider ranges, reflecting a larger sample.

Neonatal gender was evenly split, with 61 females and 63 males, similar to what was seen at NDHB and nationally.

Resuscitation at birth, of any kind, was given to 21 (16.9\%) of babies in the PROM sample and was associated with admission to SCBU $(p=0.01)$. Of the babies given any form of resuscitation, nine $(42.9 \%)$ were admitted to SCBU for respiratory support, one was admitted for other reasons, and 11 were not admitted to SCBU for any reason. The NDHB sample did not contain resuscitation data, but resuscitation occurred less often in the COMCORD sample (12.8\%). Babies in the PROM sample were more frequently resuscitated if they had not received IAP ( $28.6 \%$ vs $14.7 \%)$, though the sample did not achieve statistical significance.

Nineteen babies (15.3\%) were admitted to the SCBU (see Table 4-13), with 15 (12.1\%) admitted for respiratory support of $>4$ hours, and four (3.2\%) admitted for other reasons (such as jaundice or congenital conditions). Māori ethnicity was associated with admission to SCBU for any reason (OR 3.38, 95\% Cl, [1.22, 9.34]). Five (4\%) babies were transferred to tertiary care in a Neonatal Intensive Care Unit (NICU), three due to congenital abnormalities and two for respiratory support. NDHB reported a 9.3\% admission rate overall, and COMCORD an admission rate of 3.9\%, but neither identifies whether babies were admitted for respiratory support or other reasons.

The mean duration of admission to SCBU for the PROM sample was just over 6.5 days, though the median was two days. Three babies had 10-day admissions each, and one baby was continuously admitted to SCBU for 57 days, including a period in Neonatal Intensive Care Unit (NICU). The reasons for admission were frequently multifactorial and are summarised in Table 4-13. 
Table 4-13: Admissions to SCBU and diagnoses

\begin{tabular}{clc}
$\begin{array}{l}\text { No. of } \\
\text { babies }\end{array}$ & Reason for admission/diagnosis & $\begin{array}{c}\text { Transferred } \\
\text { to NICU? }\end{array}$ \\
\hline 1 & $\begin{array}{l}\text { Valvular pulmonary stenosis and patent ductus arteriosus to 1/12, jaundice, } \\
\text { tachypnoeoa, pustules }\end{array}$ & $\mathrm{Y}$ \\
1 & Hyponatraemia & $\mathrm{Y}$ \\
1 & Multiple organ failure and hypoxic ischemic encephalopathy stage 3, genetic & $\mathrm{Y}$ \\
& abnormality & \\
1 & Airway malformation, heart defect and vocal cord palsy & $\mathrm{Y}$ \\
1 & Stage 1 hypoxic ischemic encephalopathy, possible pneumothorax & $\mathrm{N}$ \\
2 & Presumed sepsis & $\mathrm{N}$ \\
2 & Presumed sepsis, confirmed pneumonia & $\mathrm{N}$ \\
1 & Presumed sepsis, maternal morphine dosage x 4 in labour & $\mathrm{N}$ \\
1 & Presumed sepsis, hypoglycaemia, jaundice & $\mathrm{N}$ \\
1 & Transient tachypnoea of the newborn, jaundice & $\mathrm{N}$ \\
1 & Respiratory distress syndrome, small pneumothorax. Parents had influenza. & $\mathrm{N}$ \\
1 & Persistent pulmonary hypertension & $\mathrm{N}$ \\
1 & ABO incompatibility & $\mathrm{N}$ \\
1 & Hypoglycaemia & $\mathrm{N}$ \\
3 & Observation & $\mathrm{N}$ \\
19 & Total & \\
\hline
\end{tabular}

Respiratory issues were formally identified in eight babies (6.4\%), with five cases of respiratory distress, and three cases of transient tachypnoea of the newborn. Five other babies who received respiratory support were diagnosed with other issues including airway malformation, maternal morphine excess, genetic abnormality, persistent pulmonary hypertension, and sepsis.

Sepsis was documented as suspected in six (4.8\%) babies, and blood cultures were taken for seven (5.6\%). All results were negative. Intravenous antibiotics were given to 14 (11.3\%) babies, although GBS was not diagnosed in any babies. There were no cases of meningitis but two cases of pneumonia (1.6\%). No cases of late onset GBS disease were apparent.

One baby in the PROM sample died aged three days due to genetic abnormality leading to multiple organ failure and severe hypoxic ischaemic encephalopathy. At NDHB four babies $(0.23 \%)$ died in the perinatal period, of which three were stillbirths and one was a neonatal death, unrelated to PROM. In the COMCORD sample there were 10 neonatal deaths and 42 stillbirths. It is worth noting that if the samples represented the same time period, NDHB 
(including the PROM sample births) would represent $9.6 \%$ of the national perinatal deaths but only $6.5 \%$ of the births.

The mean duration of neonatal stay for all babies in the PROM sample was 3.1 days, with a median of 3.0. The minimum stay was one day, and the maximum stay was 11 days. This variable collected only the neonatal stay in NDHB facilities, including postnatal transfers to primary centres, and excluded time spent at the tertiary NICU for any babies. NDHB did not provide neonatal stay data (other than date of admission to SCBU, if relevant) and COMCORD provided only the numbers of babies admitted to SCBU/NICU at birth.

Exclusive breastfeeding ${ }^{25}$ on discharge in the PROM sample was 107 (86.3\%) of babies which was lower than in the NDHB sample (90.5\%). No babies were recorded as 'fully' breastfed in the sample, but 13 (10.5\%) babies were partially breastfed - a significantly higher rate of supplementary feeding than in the NDHB sample (1.7\%). Formula (artificial) feeding only was used for three $(2.4 \%)$ of babies, a percentage not dissimilar to the NDHB sample. COMCORD did not provide data on breastfeeding status at discharge but did supply breastfeeding status at two weeks and at discharge from LMC, which is less useful for comparison due to a reduction in breastfeeding numbers over time.

25 “Definitions of breastfeeding (Ministry of Health, 1999):

- Exclusive breastfeeding: The infant has never, to the mother's knowledge, had any water, formula or other liquid or solid food. Only breast milk, from the breast or expressed, and prescribed* medicines have been given from birth. *Prescribed as per the Medicines Act 1981

- Fully breastfeeding: The infant has taken breast milk only, no other liquids or solids except a minimal amount of water or prescribed medicines, in the past 48 hours.

- Partial breastfeeding: The infant has taken some breast milk and some infant formula or other solid food in the past 48 hours.

- Artificial feeding: The infant has had no breast milk but has had alternative liquid such as infant formula with or without food in the past 48 hours". (Baby Friendly Hospital Initiative, 2020, p. 14) 


\subsection{Key Findings}

The following series of tables provide comparisons of 'key findings', where outcomes are compared against one key variable within the PROM sample. The key variables have been identified in order to try and identify whether any factors were of influence in managing PROM, with the exception of Māori vs non-Māori ethnicity, which was included in order to identify any issues of inequity in healthcare provision or decision-making for wāhine Māori. This section is concluded with an overall summary of outcomes for women in the PROM sample compared to the NDHB and COMCORD samples. The key variables are:

- Māori vs non-Māori ethnicity

- maternal smoking

- maternal engagement with care following ROM

- increasing duration from ROM to birth

- intrapartum oxytocin use

- use of artificial rupture of membranes

- outcomes for babies who are small for gestational age

- PROM outcomes summaries

Data is displayed consistently as number, percentages and odds ratios, with outcomes in the same order in each table ${ }^{26}$. Results with statistical significance or of interest are marked in bold. The discussion chapter will revisit each key finding topic where significant outcomes have been identified.

26 In each comparison in this section, the order of columns is (non-variable) versus (variable), however the final comparison of outcomes of all PROM women was taken from the NDHB database, and is in the opposite order of (PROM) versus (not-PROM), which provides consistency with the earlier analysis in this chapter. 


\subsubsection{Māori vs non-Māori Outcomes}

Comparative outcomes for Māori vs non-Māori women provide an interesting and thoughtprovoking contrast (Table 4-14). Wahine Māori were less likely to use epidurals, and more likely to be given IAP. Wahine Māori had a higher rate of vaginal births (with corresponding lower rates of instrumental and caesarean births), but severe postpartum haemorrhage was significantly higher for wāhine Māori. In neonatal outcomes, Māori pēpi were more likely to be admitted to SCBU for any reason, even though they were less likely to be small for gestational age. Māori pēpi were also more likely to be resuscitated following birth, have any diagnosis of respiratory distress and to receive antibiotics.

Table 4-14: Birthing outcomes for wāhine Māori

\begin{tabular}{|c|c|c|c|c|c|c|c|}
\hline \multirow[b]{2}{*}{ Variable } & \multicolumn{2}{|c|}{ Non-Māori } & \multicolumn{2}{|c|}{ Māori } & \multicolumn{3}{|c|}{$95 \% \mathrm{Cl}$} \\
\hline & $n$ & $\%$ & $n$ & $\%$ & OR & Lower & Upper \\
\hline & 76 & 100 & 47 & 100 & & & \\
\hline Any oxytocin use & 41 & 53.9 & 23 & 48.9 & 0.818 & 0.395 & 1.695 \\
\hline IAP used & 58 & 76.3 & 44 & 93.6 & 4.552 & 1.261 & 16.429 \\
\hline Chorioamnionitis (suspected) & 6 & 7.9 & 4 & 8.5 & 1.085 & 0.29 & 4.066 \\
\hline Epidural & 34 & 44.7 & 10 & 21.3 & 0.334 & 0.145 & 0.767 \\
\hline Vaginal birth & 37 & 48.7 & 36 & 76.6 & 3.45 & 1.533 & 7.765 \\
\hline Instrumental birth & 13 & 17.1 & 4 & 8.5 & 0.451 & 0.138 & 1.476 \\
\hline Caesarean birth & 26 & 34.2 & 7 & 14.9 & 0.337 & 0.132 & 0.855 \\
\hline $\mathrm{PPH}=\mathrm{Nil}$ & 50 & 65.9 & 33 & 70.2 & 1.226 & 0.559 & 2.686 \\
\hline $\mathrm{PPH}=500-999 \mathrm{mls}$ & 24 & 31.6 & 8 & 17 & 0.444 & 0.18 & 1.095 \\
\hline $\mathrm{PPH}=1000+\mathrm{mls}$ & 2 & 2.6 & 6 & 12.8 & 5.415 & 1.045 & 28.058 \\
\hline SGA & 10 & 13.2 & 4 & 8.5 & 0.614 & 0.181 & 2.083 \\
\hline Neonatal resuscitation & 11 & 14.5 & 10 & 21.3 & 1.579 & 0.62 & 4.116 \\
\hline Admission to SCBU (any) & 7 & 9.2 & 12 & 25.5 & 3.38 & 1.222 & 9.344 \\
\hline Any respiratory diagnosis & 5 & 6.6 & 8 & 17 & 2.913 & 0.892 & 9.514 \\
\hline Neonatal IV antibiotics & 7 & 9.2 & 7 & 14.9 & 1.725 & 0.564 & 5.275 \\
\hline Exclusive breastfeeding & 67 & 88.1 & 40 & 85.1 & 0.896 & 0.297 & 2.703 \\
\hline Partial breastfeeding & 7 & 9.2 & 5 & 10.6 & 1.202 & 0.358 & 4.035 \\
\hline Artificial feeding & 2 & 2.6 & 1 & 2.1 & 0.822 & 0.72 & 9.329 \\
\hline
\end{tabular}




\subsubsection{Smoking vs non-smoking}

In this comparison of outcomes for smokers vs non-smokers, four results achieved statistical significance, however there is little logic in those findings: i.e. that women who smoked were more likely to be given IAP, more likely to have a vaginal birth, less likely to have a postpartum haemorrhage and less likely to formula-feed their babies (Table 4-15). Given the small sample size, it is logical conclude the apparent differences in outcomes between smokers and non-smokers are random - although that approach undermines the validity of acceptably logical findings in other comparisons! Somewhat similarly to wāhine Māori with higher rates of IAP use, it would be interesting to examine whether there was any bias in caregiver attitudes to smokers, bearing in mind that $76.9 \%$ of the smokers were also Māori a confounding variable.

Table 4-15: Outcomes for smokers versus non-smokers

\begin{tabular}{|c|c|c|c|c|c|c|c|}
\hline \multirow[b]{2}{*}{ Variable } & \multicolumn{2}{|c|}{ Non-smoker } & \multicolumn{2}{|c|}{ Smoker } & \multicolumn{3}{|c|}{$95 \% \mathrm{Cl}$} \\
\hline & $n$ & $\%$ & $n$ & $\%$ & OR & Lower & Upper \\
\hline Total & 97 & 100 & 26 & 100 & & & \\
\hline Oxytocin used & 52 & 53.6 & 12 & 46.2 & 0.742 & 0.311 & 1.767 \\
\hline IAP & 76 & 78.4 & 26 & 100 & 1.342 & 1.198 & 1.503 \\
\hline Chorioamnionitis & 9 & 9.3 & 1 & 3.8 & 0.391 & 0.047 & 3.236 \\
\hline Epidural & 39 & 40.2 & 5 & 19.2 & 0.354 & 0.123 & 1.018 \\
\hline Vaginal & 51 & 52.6 & 22 & 84.6 & 4.961 & 1.591 & 15.472 \\
\hline Instrumental & 15 & 15.4 & 2 & 7.7 & 0.456 & 0.97 & 2.133 \\
\hline Caesarean & 31 & 32 & 2 & 7.7 & 0.177 & 0.039 & 0.799 \\
\hline $\mathrm{PPH}>500 \mathrm{mls}$ & 61 & 62.9 & 22 & 84.6 & 3.246 & 1.036 & 10.172 \\
\hline PPH 500-999mls & 29 & 29.9 & 3 & 11.5 & 0.306 & 0.085 & 1.099 \\
\hline PPH 1000mls+ & 7 & 7.2 & 1 & 3.8 & 0.514 & 0.06 & 4.378 \\
\hline SGA & 10 & 10.3 & 4 & 15.4 & 1.582 & 0.453 & 5.523 \\
\hline Neonatal resuscitation & 16 & 16.5 & 5 & 19.2 & 1.205 & 0.396 & 3.668 \\
\hline Any admission to SCBU & 15 & 15.5 & 4 & 15.4 & 0.994 & 0.3 & 3.297 \\
\hline Respiratory diagnosis & 11 & 11.3 & 2 & 7.9 & 0.652 & 0.135 & 3.141 \\
\hline Neonatal IV antibiotics & 13 & 13.4 & 1 & 3.8 & 0.258 & 0.32 & 2.074 \\
\hline Exclusive breastfeeding & 84 & 87.5 & 23 & 12 & 1.095 & 0.285 & 4.211 \\
\hline Partial breastfeeding & 9 & 9.4 & 3 & 12 & 1.261 & 0.316 & 5.037 \\
\hline Artificial feeding & 3 & 3.1 & 0 & 0 & 0.782 & 0.711 & 0.859 \\
\hline
\end{tabular}




\subsubsection{Impact of engagement of care following ROM}

The analysis below (Table 4-16) looked at outcomes for women with documented engagement with care, versus those who didn't appear to engage with care prior to labour commencing. This analysis found no statistical significance for any of the key outcomes, though it is acknowledged that documentation issues could belie any findings and numbers are small. In this analysis, women's self-knowledge and management (and unrecorded community midwifery attendance) appears to have been as protective as pre-emptive attention given within NDHB Maternity facilities.

Table 4-16: Outcomes based on engagement with care

\begin{tabular}{|c|c|c|c|c|c|c|c|}
\hline & \multicolumn{2}{|c|}{ Didn't engage } & \multicolumn{2}{|c|}{ Engaged } & \multicolumn{3}{|c|}{$95 \% \mathrm{Cl}$} \\
\hline & $\mathrm{n}$ & $\%$ & $\mathrm{n}$ & $\%$ & OR & Lower & Upper \\
\hline $\mathrm{N}(\%)$ & 50 & 100 & 73 & 100 & & & \\
\hline Oxytocin used & 24 & 48 & 40 & 54.8 & 1.313 & 0.638 & 2.701 \\
\hline IAP used & 41 & 82 & 61 & 83.6 & 1.116 & 0.431 & 2.887 \\
\hline Chorioamnionitis (suspected) & 4 & 8 & 6 & 8.2 & 1.03 & 0.275 & 3.854 \\
\hline Epidural & 15 & 30 & 29 & 39.7 & 1.538 & 0.715 & 3.306 \\
\hline Vaginal birth & 32 & 64 & 41 & 56.1 & 0.721 & 0.344 & 1.51 \\
\hline Instrumental birth & 5 & 10 & 12 & 16.4 & 1.77 & 0.582 & 5.384 \\
\hline Caesarean birth & 13 & 26 & 20 & 27.4 & 1.074 & 0.476 & 2.426 \\
\hline $\mathrm{PPH}=\mathrm{Nil}$ & 31 & 62 & 52 & 71.2 & 1.518 & 0.707 & 3.256 \\
\hline $\mathrm{PPH}=500-999 \mathrm{mls}$ & 14 & 28 & 18 & 24.7 & 0.842 & 0.372 & 1.901 \\
\hline $\mathrm{PPH}=1000+\mathrm{mls}$ & 5 & 10 & 3 & 4.1 & 0.386 & 0.088 & 1.694 \\
\hline SGA & 6 & 12 & 8 & 11 & 0.903 & 0.293 & 2.782 \\
\hline Neonatal resuscitation & 8 & 16 & 13 & 17.8 & 1.138 & 0.433 & 2.986 \\
\hline Admission to SCBU (any) & 9 & 18 & 10 & 13.7 & 0.723 & 0.271 & 1.932 \\
\hline Any respiratory diagnosis & 7 & 14 & 6 & 8.2 & 0.55 & 0.173 & 1.747 \\
\hline Neonatal IV antibiotics & 6 & 12 & 8 & 11 & 0.903 & 0.293 & 2.782 \\
\hline Exclusive breastfeeding & 41 & 82 & 66 & 90.4 & 1.84 & 0.621 & 5.454 \\
\hline Partial breastfeeding & 7 & 14 & 5 & 6.8 & 0.441 & 0.132 & 1.48 \\
\hline Artificial feeding & 1 & 2 & 2 & 2.7 & 1.352 & 0.119 & 15.331 \\
\hline
\end{tabular}




\subsubsection{Did increasing duration of ROM to birth affect outcomes?}

This section presents three tables displaying varying methods to divide findings by time allocations. It starts with births occurring in 12-hour intervals, and then compares larger groupings to consider outcomes for those birthing after 48 hours and then 96 hours.

The first analysis does not include odds-ratio calculations, but presents percentages for women, grouped into 12-hour intervals who experienced each variable (Table 4-18). There does not appear to be any trends within each variable or period, and the small numbers of women who birthed from 48 to 95:59 hours appear likely insufficient to observe any reliable effects of duration. The women who birthed after 96 hours had high use of oxytocin, but low use of epidural, high incidence of vaginal birth, low rates of postpartum haemorrhage and high rates of exclusive breastfeeding - generally a comparatively positive picture for prolonged expectant management, if it were not for concerns for the neonate. Of neonates born after $96+$ hours of ROM, $23.1 \%$ were resuscitated and subsequently admitted to SCBU - though actual numbers were small.

Table 4-17: Outcomes by duration from ROM to birth

\begin{tabular}{|c|c|c|c|c|c|c|c|c|}
\hline & \multicolumn{8}{|c|}{ Hours (\% of each row variable) } \\
\hline & 18 to $23: 59$ & 24 to $35: 59$ & 36 to $47: 59$ & 48 to $59: 59$ & 60 to $71: 59$ & 72 to $83: 59$ & 84 to $95: 59$ & $96+$ \\
\hline$n=$ & 27 & 36 & 21 & 7 & 5 & 7 & 7 & 13 \\
\hline Oxytocin used & 55.5 & 47.2 & 42.8 & 42.8 & 60.0 & 57.1 & 71.4 & 61.5 \\
\hline IAP used & 55.5 & 88.9 & 100.0 & 100.0 & 80.0 & 71.4 & 85.7 & 92.3 \\
\hline Chorioamnionitis (suspected) & 7.4 & 13.9 & 0.0 & 0.0 & 0.0 & 0.0 & 14.3 & 15.4 \\
\hline Epidural & 44.4 & 25.0 & 38.1 & 57.1 & 40.0 & 14.3 & 71.4 & 23.1 \\
\hline Vaginal birth & 44.4 & 61.1 & 76.2 & 42.9 & 100.0 & 42.9 & 14.3 & 84.6 \\
\hline Instrumental birth & 22.2 & 16.7 & 0.0 & 28.6 & 0.0 & 14.3 & 28.6 & 0.0 \\
\hline Caesarean birth & 33.3 & 22.2 & 23.8 & 28.6 & 0.0 & 42.9 & 57.1 & 15.4 \\
\hline $\mathrm{PPH}=\mathrm{Nil}$ & 70.4 & 66.7 & 66.7 & 71.4 & 100.0 & 28.5 & 42.9 & 84.6 \\
\hline $\mathrm{PPH}=500-999 \mathrm{mls}$ & 22.2 & 25.0 & 28.6 & 14.3 & 0.0 & 57.1 & 57.1 & 15.4 \\
\hline $\mathrm{PPH}=1000+\mathrm{mls}$ & 7.4 & 8.3 & 4.7 & 14.3 & 0.0 & 14.3 & 0.0 & 0.0 \\
\hline Neonatal resuscitation & 14.9 & 22.2 & 14.3 & 0.0 & 20.0 & 14.3 & 14.3 & 23.1 \\
\hline Admission to SCBU (any) & 7.4 & 22.2 & 9.5 & 14.3 & 0.0 & 28.6 & 14.3 & 23.1 \\
\hline Any respiratory diagnosis & 7.4 & 13.9 & 9.5 & 14.3 & 0.0 & 28.6 & 14.3 & 0.0 \\
\hline Neonatal IV antibiotics & 7.4 & 16.7 & 9.5 & 14.3 & 0.0 & 28.6 & 14.3 & 0.0 \\
\hline Exclusive breastfeeding & 92.6 & 72.2 & 90.5 & 100 & 80.0 & 85.7 & 71.4 & 92.3 \\
\hline Partial breastfeeding & 3.7 & 16.7 & 4.8 & 0 & 20.0 & 14.3 & 28.6 & 7.7 \\
\hline Artificial feeding & 3.7 & 2.8 & 4.7 & 0 & 0 & 0 & 0 & 0 \\
\hline
\end{tabular}


Two comparisons of odds-ratios based on duration at intervals of over 48 hours and over 96 hours are presented next, to demonstrate as clearly as possible with this data whether any issues were found with prolonged ROM.

In the previous comparison, outcomes based on duration were difficult to compare due to low numbers at durations of over 48 hours. Assuming 48 hours would be considered a reasonable period for birth to occur following a ROM event (allowing 24 hours for expectant management and up to 24 hours to deliver either spontaneously or after induction of labour), the analysis in Table 4-18 considers outcomes for women birthing prior to versus after 48 or 96 hours post-ROM.

Table 4-18: Outcomes for women with under or over 48 hours duration from ROM to birth

\begin{tabular}{|c|c|c|c|c|c|c|c|}
\hline \multirow[b]{2}{*}{ Variable } & \multicolumn{2}{|c|}{$<48$ hours } & \multicolumn{2}{|c|}{$>48$ hours } & \multicolumn{3}{|c|}{$95 \% \mathrm{Cl}$} \\
\hline & $n$ & $\%$ & $n$ & $\%$ & OR & Lower & Upper \\
\hline & 84 & 100 & 39 & 100 & & & \\
\hline Oxytocin used & 41 & 48.8 & 23 & 59 & 1.508 & 0.699 & 3.25 \\
\hline IAP used & 68 & 81 & 34 & 87.2 & 1.6 & 0.54 & 4.736 \\
\hline Chorioamnionitis (suspected) & 7 & 7 & 3 & 7.6 & 0.917 & 0.224 & 3.752 \\
\hline Epidural & 29 & 34.5 & 15 & 38.5 & 1.185 & 0.54 & 2.602 \\
\hline Vaginal birth & 50 & 59.5 & 23 & 59 & 0.978 & 0.451 & 2.117 \\
\hline Instrumental birth & 12 & 14.3 & 5 & 12.8 & 0.882 & 0.288 & 2.705 \\
\hline Caesarean birth & 22 & 26.2 & 11 & 28.2 & 1.107 & 0.473 & 2.591 \\
\hline $\mathrm{PPH}=\mathrm{Nil}$ & 57 & 67.9 & 26 & 66.7 & 0.947 & 0.422 & 2.125 \\
\hline $\mathrm{PPH}=500-999 \mathrm{mls}$ & 21 & 25 & 11 & 28.2 & 1.179 & 0.501 & 2.77 \\
\hline $\mathrm{PPH}=1000+\mathrm{mls}$ & 6 & 7.1 & 2 & 5.1 & 0.703 & 0.135 & 3.65 \\
\hline Neonatal resuscitation & 15 & 17.9 & 6 & 15.4 & 0.836 & 0.298 & 2.351 \\
\hline Admission to SCBU (any) & 12 & 14.3 & 7 & 17.9 & 1.313 & 0.473 & 3.643 \\
\hline Any respiratory diagnosis & 9 & 10.7 & 4 & 10.3 & 0.952 & 0.274 & 3.305 \\
\hline Neonatal IV antibiotics & 10 & 11.9 & 4 & 10.3 & 0.846 & 0.248 & 2.885 \\
\hline Exclusive breastfeeding & 73 & 86.9 & 34 & 87.2 & 1.025 & 0.33 & 3.181 \\
\hline Partial breastfeeding & 8 & 9.5 & 5 & 12.8 & 1.397 & 0.426 & 4.584 \\
\hline Artificial feeding & 3 & 3.6 & 0 & 0 & 0.675 & 0.596 & 0.764 \\
\hline
\end{tabular}

For women birthing before 48 hours post-ROM, there were no statistically significant outcomes that differed, compared to women who had birthed later, and percentages varied little between the two groups. The next table compares outcomes for women birthing prior to and after 96 hours post-ROM.

The analysis of outcomes for women birthing prior to, versus after 96 hours post-ROM, does provide statistically significant outcomes, however those statistical significances are only 
present for outcomes where no cases in the over-96 hours group had a specific outcome (Table 4-19). There was a higher incidence of IAP use and vaginal birth, and fewer epidurals and caesarean births. The percentages of babies resuscitated ( $16.4 \%$ vs $23.1 \%$ ) and admitted to SCBU (14.5\% vs $23.1 \%)$ was relatively and concerningly high for both groups although the number of babies in the over 96 hours group was small, and those individual babies did not have ongoing respiratory or septic issues.

Table 4-19: Outcomes for women with duration of under or over 96 hours from ROM to birth

\begin{tabular}{|c|c|c|c|c|c|c|c|}
\hline \multirow[b]{2}{*}{ Variable } & \multicolumn{2}{|c|}{$<96$ hours } & \multicolumn{2}{|c|}{$>96$ hours } & \multirow[b]{2}{*}{ OR } & \multicolumn{2}{|c|}{$95 \% \mathrm{Cl}$} \\
\hline & $n$ & $\%$ & $n$ & $\%$ & & Lower & Upper \\
\hline Total & 110 & 100.0 & 13 & 100.0 & & & \\
\hline Oxytocin used & 56 & 50.9 & 8 & 61.5 & 1.543 & 0.475 & 5.012 \\
\hline IAP used & 90 & 81.8 & 12 & 92.3 & 2.667 & 0.328 & 21.706 \\
\hline Chorioamnionitis (suspected) & 8 & 7.3 & 2 & 15.4 & 2.318 & 0.437 & 12.311 \\
\hline Epidural & 41 & 37.3 & 3 & 23.1 & 0.505 & 0.131 & 1.941 \\
\hline Vaginal birth & 62 & 56.4 & 11 & 84.6 & 4.258 & 0.901 & 20.123 \\
\hline Instrumental birth & 17 & 15.5 & 0 & 0.0 & 0.877 & 0.817 & 0.942 \\
\hline Caesarean birth & 31 & 28.2 & 2 & 15.4 & 0.463 & 0.097 & 2.211 \\
\hline $\mathrm{PPH}=\mathrm{Nil}$ & 72 & 65.5 & 11 & 84.6 & 2.903 & 0.612 & 13.773 \\
\hline $\mathrm{PPH}=500-999 \mathrm{mls}$ & 30 & 27.3 & 2 & 15.4 & 0.485 & 0.101 & 2.316 \\
\hline $\mathrm{PPH}=1000+\mathrm{mls}$ & 8 & 7.3 & 0 & 0.0 & 0.887 & 0.831 & 0.947 \\
\hline Neonatal resuscitation & 18 & 16.4 & 3 & 23.1 & 1.533 & 0.384 & 6.129 \\
\hline Admission to SCBU (any) & 16 & 14.5 & 3 & 23.1 & 1.763 & 0.437 & 7.111 \\
\hline Any respiratory diagnosis & 13 & 11.8 & 0 & 0.0 & 0.882 & 0.824 & 0.944 \\
\hline Neonatal IV antibiotics & 14 & 12.7 & 0 & 0.0 & 0.881 & 0.822 & 0.944 \\
\hline Exclusive breastfeeding & 95 & 86.4 & 12 & 92.3 & 1.895 & 0.229 & 15.651 \\
\hline Partial breastfeeding & 12 & 10.9 & 1 & 7.7 & 0.681 & 0.081 & 5.705 \\
\hline Artificial feeding & 3 & 2.7 & 0 & 0.0 & 0.892 & 0.838 & 0.949 \\
\hline
\end{tabular}

These two analyses found no truly significant relationships between increasing duration of ROM over 48 hours and over 96 hours with increased risk of undesired outcomes for women or babies. Simple percentages show a different perspective though - percentages of women who birthed after 96 hours post-ROM were higher regarding use of IAP, vaginal birth, postpartum haemorrhage, and exclusive breastfeeding, and women used less epidural pain relief. From this analysis it would be tempting to say that waiting for over 96 hours post-ROM may have improved outcomes for some women, however the numbers were too low to make such an assertion with confidence. 


\subsubsection{Did an artificial rupture of the membranes affect outcomes?}

Artificial rupture of the membranes following confirmed prelabour rupture of membranes event occurred in 24 (19.5\%) women (?

Table 4-20). ARM was clearly associated with oxytocin use, and given that 20 of the 24 women experienced ARM as 'augmentation', it was probable in many cases that oxytocin use preceded the ARM, which is anecdotally an well-worn trope in caring for women with PROM - it has been assumed that membranes have ruptured but oxytocin infusion does not increase cervical dilatation, and membranes containing forewaters can be detected during a digital vaginal examination, leading to an ARM. Epidural use and caesarean births were more frequent for women following ARM, but not significantly so, and likely related to oxytocin use. The finding that women given an ARM were more likely to birth a small for gestational age baby is interesting - perhaps the physiological mechanisms of labour were less efficient for small babies at term?

Table 4-20: Outcomes where ARM was used vs Nil ARM

\begin{tabular}{|c|c|c|c|c|c|c|c|}
\hline \multirow[b]{2}{*}{ Variable } & \multicolumn{2}{|c|}{ Nil ARM use } & \multicolumn{2}{|c|}{ ARM used } & \multirow[b]{2}{*}{ OR } & \multicolumn{2}{|c|}{$95 \% \mathrm{Cl}$} \\
\hline & $n$ & $\%$ & $\mathrm{n}$ & $\%$ & & Lower & Upper \\
\hline & 99 & 100 & 24 & 100 & & & \\
\hline Oxytocin used & 47 & 47.7 & 17 & 70.8 & 2.687 & 1.024 & 7.049 \\
\hline IAP & 81 & 81.9 & 21 & 87.5 & 1.556 & 0.418 & 5.783 \\
\hline Chorioamnionitis & 9 & 9.1 & 1 & 4.2 & 0.435 & 0.52 & 3.608 \\
\hline Epidural & 33 & 33.3 & 11 & 45.8 & 1.692 & 0.684 & 4.184 \\
\hline Vaginal & 60 & 60.6 & 13 & 54.2 & 0.768 & 0.313 & 1.887 \\
\hline Instrumental & 15 & 15.2 & 2 & 8.3 & 0.509 & 0.108 & 2.394 \\
\hline Caesarean & 24 & 24.2 & 9 & 37.5 & 1.875 & 0.728 & 4.827 \\
\hline $\mathrm{PPH}>500 \mathrm{mls}$ & 67 & 67.7 & 16 & 66.7 & 0.955 & 0.37 & 2.464 \\
\hline PPH 500-999mls & 26 & 26.3 & 6 & 25 & 0.936 & 0.335 & 2.613 \\
\hline PPH 1000mls+ & 6 & 6.1 & 2 & 8.3 & 1.409 & 0.266 & 7.458 \\
\hline SGA & 8 & 8.1 & 6 & 25 & 3.792 & 1.173 & 12.254 \\
\hline Neonatal resuscitation & 17 & 17.2 & 4 & 16.7 & 0.965 & 0.292 & 3.183 \\
\hline Any admission to SCBU & 17 & 17.2 & 2 & 8.3 & 0.439 & 0.94 & 2.043 \\
\hline Respiratory diagnosis & 11 & 11.1 & 2 & 8.3 & 0.727 & 0.15 & 3.522 \\
\hline Neonatal IV antibiotics & 13 & 13.1 & 1 & 4.2 & 0.288 & 0.36 & 2.315 \\
\hline Exclusive breastfeeding & 87 & 87.9 & 20 & 83.3 & 0.632 & 0.182 & 2.192 \\
\hline Partial breastfeeding & 9 & 9.1 & 3 & 12.5 & 1.413 & 0.352 & 5.674 \\
\hline Artificial feeding & 2 & 2 & 1 & 4.2 & 2.087 & 0.181 & 24.02 \\
\hline
\end{tabular}




\subsubsection{Did oxytocin use in labour affect outcomes?}

The findings in this analysis are comparatively clear (Table 4-21). Oxytocin use in labour was positively associated with increased use of epidural and instrumental birth, and correspondingly negatively associated with vaginal birth. There was also an apparent relationship between blood-loss and oxytocin use, with women who had used oxytocin more likely to experience postpartum haemorrhage, however significance was not established except to prove a negative relationship with a nil postpartum haemorrhage. A sub-analysis comparing non-postpartum haemorrhage to any postpartum haemorrhage established significance (OR 2.582, 95\% Cl, [1.171, 5.693]).

Table 4-21: Outcomes by oxytocin use versus nil oxytocin use

\begin{tabular}{lccccccccc}
\hline \multirow{2}{*}{ Variable } & \multicolumn{2}{c}{ Nil oxytocin } & & \multicolumn{2}{c}{ Oxytocin } & & \multicolumn{3}{c}{$95 \% \mathrm{Cl}$} \\
\cline { 2 - 3 } & $n$ & $\%$ & & $n$ & $\%$ & & OR & Lower & Upper \\
\hline Total & 59 & 100 & & 64 & 100 & & & \\
IAP & 46 & 78 & & 56 & 87.5 & & 1.978 & 0.755 & 5.183 \\
Chorioamnionitis (suspected) & 3 & 5.1 & & 7 & 10.9 & & 2.292 & 0.564 & 9.314 \\
Epidural & $\mathbf{9}$ & 15.3 & & $\mathbf{3 5}$ & $\mathbf{5 4 . 7}$ & & $\mathbf{6 . 7 0 5}$ & $\mathbf{2 . 8 2 7}$ & $\mathbf{1 5 . 9 0 3}$ \\
Vaginal birth & $\mathbf{4 3}$ & 72.9 & & $\mathbf{3 0}$ & $\mathbf{4 6 . 9}$ & & $\mathbf{0 . 3 2 8}$ & $\mathbf{0 . 1 5 4}$ & $\mathbf{0 . 6 9 9}$ \\
Instrumental birth & $\mathbf{1}$ & 1.7 & & $\mathbf{1 6}$ & $\mathbf{2 5}$ & & $\mathbf{1 9 . 3 3 3}$ & $\mathbf{2 . 2 4 7 4}$ & $\mathbf{1 5 1 . 1 0 8}$ \\
Caesarean birth & 15 & 25.4 & & 18 & 28.1 & & 1.148 & 0.516 & 2.555 \\
PPH = Nil & $\mathbf{4 6}$ & 78 & & $\mathbf{3 7}$ & $\mathbf{5 7 . 8}$ & & $\mathbf{0 . 3 8 7}$ & $\mathbf{0 . 1 7 6}$ & $\mathbf{0 . 8 5 4}$ \\
PPH = 500-999mls & 12 & 20.3 & & 20 & 31.3 & & 1.78 & 0.78 & 4.064 \\
PPH = 1000+mls & 1 & 1.7 & & 7 & 10.9 & & 7.123 & 0.849 & 59.754 \\
SGA & 8 & 13.6 & & 6 & 9.4 & & 0.659 & 0.214 & 2.028 \\
Neonatal resuscitation & 9 & 15.3 & & 12 & 18.6 & & 1.282 & 0.497 & 3.307 \\
Admission to SCBU (any) & 10 & 16.9 & & 9 & 14.1 & & 0.802 & 0.301 & 2.135 \\
Any respiratory diagnosis & 6 & 10.2 & & 7 & 10.9 & & 1.085 & 0.343 & 3.436 \\
Neonatal IV antibiotics & 6 & 10.2 & & 8 & 12.5 & & 1.262 & 0.41 & 3.88 \\
Exclusive breastfeeding & 52 & 88.1 & & 55 & 86 & & 0.925 & 0.313 & 2.733 \\
Partial breastfeeding & 5 & 8.5 & & 7 & 10.9 & & 1.35 & 0.404 & 4.514 \\
Artificial feeding & 2 & 3.4 & & 1 & 1.6 & & 0.46 & 0.041 & 5.207 \\
\hline
\end{tabular}




\subsubsection{Did neonates who were small for gestational age have different outcomes?}

The only statistically significant outcome for births of small for gestational age babies (under the $10^{\text {th }}$ centile for weight) was a negative association with severe postpartum haemorrhage - though again, the sample was too small for confidence (Table 4-22). However, most births of small for gestational age babies had no postpartum haemorrhage at all. Use of epidural was reduced in women with small for gestational age babies, and vaginal births were more frequent, however not significantly. Admissions to SCBU were over double those of nonsmall for gestational age babies; however, did not achieve statistical significance in this small sample.

Table 4-22: Outcomes for small for gestational age babies

\begin{tabular}{|c|c|c|c|c|c|c|c|}
\hline \multirow[b]{2}{*}{ Variable } & \multicolumn{2}{|c|}{ non-SGA } & \multicolumn{2}{|c|}{ SGA } & \multirow[b]{2}{*}{ OR } & \multicolumn{2}{|c|}{$95 \% \mathrm{Cl}$} \\
\hline & $n$ & $\%$ & $n$ & $\%$ & & Lower & Upper \\
\hline Total & 109 & 100 & 14 & 100 & & & \\
\hline Oxytocin used & 58 & 53.2 & 6 & 42.9 & 0.659 & 0.214 & 2.028 \\
\hline IAP used & 91 & 83.5 & 11 & 78.6 & 0.725 & 0.184 & 2.863 \\
\hline Chorioamnionitis (suspected) & 7 & 6.4 & 3 & 21.4 & 3.974 & 0.897 & 17.61 \\
\hline Epidural & 42 & 38.5 & 2 & 14.3 & 0.266 & 0.057 & 1.247 \\
\hline Vaginal birth & 63 & 57.8 & 10 & 71.4 & 1.825 & 0.539 & 6.184 \\
\hline Instrumental birth & 16 & 14.7 & 1 & 7.1 & 0.447 & 0.055 & 3.658 \\
\hline Caesarean birth & 30 & 27.5 & 3 & 21.4 & 0.718 & 0.187 & 2.754 \\
\hline $\mathrm{PPH}=\mathrm{Nil}$ & 70 & 64.2 & 13 & 92.9 & 7.243 & 0.913 & 57.474 \\
\hline $\mathrm{PPH}=500-999 \mathrm{mls}$ & 31 & 28.4 & 1 & 7.1 & 0.194 & 0.024 & 1.543 \\
\hline $\mathrm{PPH}=1000+\mathrm{mls}$ & 8 & 7.4 & 0 & 0 & 0.878 & 0.82 & 0.94 \\
\hline Neonatal resuscitation & 18 & 16.5 & 3 & 21.4 & 1.264 & 0.324 & 4.936 \\
\hline Admission to SCBU (any) & 15 & 13.8 & 4 & 28.6 & 2.279 & 0.642 & 8.094 \\
\hline Any respiratory diagnosis & 12 & 11 & 1 & 7.1 & 0.577 & 0.07 & 4.789 \\
\hline Neonatal IV antibiotics & 12 & 11 & 2 & 14.3 & 1.244 & 0.25 & 6.19 \\
\hline Exclusive breastfeeding & 95 & 87.2 & 12 & 85.7 & 0.884 & 0.179 & 4.374 \\
\hline Partial breastfeeding & 11 & 10.1 & 2 & 14.3 & 1.485 & 0.293 & 7.514 \\
\hline Artificial feeding & 3 & 2.8 & 0 & 0 & 0.883 & 0.828 & 0.943 \\
\hline
\end{tabular}




\subsubsection{Outcomes for women with PROM as a factor in their labour}

Having explored many variables in seeking to find key factors of influence for women experiencing PROM, there have been many points of interest, but ultimately few findings with statistical significance. Table 4-23 below provides a comparison of the outcomes for women in the PROM sample versus those in the full NDHB sample, where the outcomes with significant odds ratios are marked in bold. Not all 'key findings' variables are able to be included due to limitations in the NDHB data, however comparisons have been presented for all which are available.

Table 4-23: PROM outcome comparisons vs NDHB outcomes

\begin{tabular}{|c|c|c|c|c|c|c|c|}
\hline \multirow[b]{2}{*}{ Variable } & \multicolumn{2}{|c|}{ PROM } & \multicolumn{2}{|c|}{ NDHB } & \multicolumn{3}{|c|}{$95 \% \mathrm{Cl}$} \\
\hline & $n$ & $\%$ & $n$ & $\%$ & OR & Lower & Upper \\
\hline Total & $123 *$ & 100 & 1717 & 100 & & & \\
\hline Induction & 34 & 27.4 & 332 & 19.3 & 1.588 & 1.051 & 2.399 \\
\hline Augmentation & 70 & 56.9 & 404 & 23.5 & 4.306 & 2.963 & 6.259 \\
\hline Any oxytocin use in labour & 64 & 52 & 181 & 10.5 & 9.205 & 6.259 & 13.539 \\
\hline Epidural & 44 & 35.8 & 253 & 14.7 & 3.208 & 2.168 & 4.748 \\
\hline Vaginal birth & 73 & 59.3 & 1279 & 74.5 & 0.5 & 0.343 & 0.728 \\
\hline Instrumental birth & 17 & 13.8 & 107 & 6.2 & 2.458 & 1.42 & 4.255 \\
\hline Caesarean birth & 33 & 26.8 & 331 & 19.3 & 1.538 & 1.014 & 2.332 \\
\hline Bloodloss $=$ below $500 \mathrm{mls}$ & 84 & 68.3 & 1442 & 84 & 0.441 & 0.296 & 0.658 \\
\hline $\mathrm{PPH}=$ above $500 \mathrm{mls}$ & 32 & 26 & 249 & 14.5 & 2.103 & 1.375 & 3.216 \\
\hline $\mathrm{PPH}=$ above $750 \mathrm{mls}$ & 7 & 5.7 & 46 & 2.7 & 2.192 & 0.968 & 4.963 \\
\hline Admission to SCBU (any) & 19 & 15.4 & 157 & 9.1 & 2.453 & 1.454 & 4.14 \\
\hline Exclusive Breastfeeding & 107 & 87 & 1554 & 90.5 & 0.538 & 0.308 & 0.938 \\
\hline Fully Breastfeeding & 0 & 0 & 47 & 2.7 & 0.972 & 0.964 & 0.98 \\
\hline Partial Breastfeeding & 13 & 10.6 & 29 & 1.7 & 6.724 & 3.4 & 13.3 \\
\hline Artificial Feeding & 3 & 2.4 & 49 & 2.9 & 0.832 & 0.255 & 2.707 \\
\hline
\end{tabular}

*Twin II is excluded from all calculations in this series

The preceding key outcome comparisons made it clear that generally, it was not possible to point to any one variable and attribute cause, therefore supporting the practice of informed consent and for women to participate in their own decision-making. The overall picture presented in this comparison is that PROM itself was associated with increased risks for women, and this analysis found statistical significance in most of the key outcomes included. Women experiencing PROM of over 18 hours had higher rates of labour induction, augmentation, oxytocin use in labour, use of epidural, instrumental and caesarean births, 
blood loss of over $500 \mathrm{mls}$, neonatal admission to SCBU for any reason, and increased incidence of partial breastfeeding over decreases in exclusive and fully breastfeeding.

The following comparison made using the COMCORD sample of women birthing with PROM $\geq 18$ hours, excluding all inductions of labour (as it isn't recorded which event occurred first), similarly found much higher rates of oxytocin and epidural use, reduced vaginal births, increased instrumental births, increased postpartum haemorrhage, neonatal resuscitation and admissions to SCBU (Table 4-24). The percentages for each of these categories bear striking resemblance to those found in the PROM and NDHB sample data for women with PROM $\geq 18$ hours. This further supports the theory that PROM does increase risks for women and babies, but the NDHB PROM policy is as safe for women and babies as practice throughout New Zealand, and meets their needs by supporting informed decision-making and consent processes for expectant management of up to 96 hours. The COMCORD figures support the theory that clinical practice and populations have changed since the Hannah et al. (1996) trial, and new research is now needed.

Table 4-24: PROM outcomes comparisons for COMCORD sample of PROM $\geq 18 \mathrm{hrs}$ with no induction of labour

\begin{tabular}{|c|c|c|c|c|}
\hline \multirow[b]{2}{*}{ Variable } & \multicolumn{2}{|c|}{ PROM, no IOL } & \multicolumn{2}{|c|}{ All others } \\
\hline & $n$ & $\%$ & $n$ & $\%$ \\
\hline Total & 1548 & 100 & 25952 & 100 \\
\hline Any oxytocin use in labour & 539 & 34.8 & 3145 & 12.1 \\
\hline Epidural & 552 & 35.7 & 4376 & 16.9 \\
\hline Vaginal birth & 900 & 58 & 18089 & 69.7 \\
\hline Instrumental birth & 248 & 16 & 2021 & 7.8 \\
\hline Caesarean birth & 400 & 25.8 & 5840 & 22.5 \\
\hline Bloodloss $=$ below $500 \mathrm{mls}$ & 1182 & 76.4 & 20864 & 80.4 \\
\hline $\mathrm{PPH}=501-750 \mathrm{mls}$ & 141 & 9.1 & 1822 & 7 \\
\hline$P P H=750-1000 \mathrm{mls}$ & 107 & 6.9 & 1402 & 5.4 \\
\hline $\mathrm{PPH}=>1000 \mathrm{mls}$ & 70 & 4.5 & 1099 & 4.2 \\
\hline Neonatal resuscitation & 231 & 14.9 & 3158 & 12.2 \\
\hline Admission to SCBU (any) & 120 & 7.8 & 1439 & 5.5 \\
\hline Exclusive Breastfeeding* & 1081 & 69.8 & 18028 & 69.5 \\
\hline Fully Breastfeeding & 122 & 7.9 & 1915 & 7.4 \\
\hline Partial Breastfeeding & 203 & 13.1 & 3412 & 13.1 \\
\hline Artificial Feeding & 82 & 5.3 & 1844 & 7.1 \\
\hline
\end{tabular}

*all breastfeeding results are from 2 weeks postpartum 


\subsection{Summary}

This chapter has presented demographic, antenatal, intrapartum, postnatal and neonatal outcomes for women and their babies who have birthed following PROM. Several key outcomes have been examined from many angles, often without finding significant factors which have influenced outcomes for the women in this group. Women and babies were significantly more likely to experience labours requiring the use of medicines, used pain relief more often, were less likely to spontaneously birth vaginally, were more likely to experience postpartum haemorrhage, their babies were more likely to be admitted to SCBU and were less likely to be exclusively or fully breastfed. The conclusion that can be drawn is that PROM itself was the largest risk factor for women birthing following PROM, and that factors such as maternal smoking, small for gestational age babies or prolonged duration had little bearing on outcomes. The next chapter will examine the findings in this chapter further, and discuss perspectives on the issues raised by this data. 


\section{Discussion}

\subsection{Introduction}

Prelabour rupture of membranes at term can be challenging to manage and may involve multidisciplinary consultation and care for the mother and her baby. Practice guidance is provided separately by each of the 20 DHBs in New Zealand, despite national and international consensus statements. NDHB has developed a localised approach which differs from common New Zealand practice advice. At NDHB expectant management is enabled for up to 96 hours, compared with most other DHB policies that recommend expectant management of 24 hours or less. This outcomes evaluation used a clinical records review of a one-year sample of NDHB paper files and electronic records pertaining to women who had experienced PROM of 18 hours or more, and subsequently birthed within NDHB facilities. This study aimed to assess whether:

1. the NDHB PROM policy was applied in practice?

2. this policy met the needs of birthing women experiencing PROM?

3. the current is policy safe for women and babies?

This discussion chapter highlights and explores the salient findings from the data and considers whether the aims of the study have been met.

The first aim of this study was to observe if the NDHB PROM policy is applied in practice. It was estimated that $4.8 \%$ to $6 \%$ of all births would be affected by PROM at term. The initial NDHB data download included all births from 37 weeks gestation, of which there were 1840 births. The final PROM sample included 123 women who had PROM of 18 or more hours, representing $6.7 \%$ of NDHB births for a one-year period, therefore the number sought for inclusion was achieved. The PROM policy recommends obstetric consultation after 24 hours of PROM, which was documented as occurring for $52.8 \%$ of women in the PROM sample. The policy then recommends induction of labour as clinically indicated or elected by each woman, or expectant management of up to 96 hours for low risk women. Induction of labour was documented as offered to $30.1 \%$ of women and was used for $27.6 \%$ of women. 
The other $72.4 \%$ of women in the PROM sample did not need induction of labour and can therefore fit the definition of using expectant management for up to 96 hours. Of the 13 women who reached 96 hours, ten then had labour induced, one had labour augmented, and two arrived at a facility in labour and birthed spontaneously. Therefore, it appears that the NDHB PROM policy appears to be adhered to. Clinical record reviews depend upon what is written, and it appears that for many women, conversations around this event that were held were either not documented or not documented well. Whether women were engaged in decision-making is much harder to speak to, as much documentation omitted any description of the information given to women and their responses.

This chapter will now provide explore whether women's needs were met through the discussion of the findings, and return to the remaining aims of assessing the safety and equity of the PROM policy for women and their babies at the end of the chapter.

\subsection{Demographics}

Demographically, the PROM sample was reasonably similar to the NDHB population, though had a lower proportion of Māori and Pasifika women, and a greater number of primiparous women. The COMCORD sample showed greater diversity of ethnicities. Why Māori and Pasifika were under-represented in the PROM sample is unknown. It is suggested there is possibly a Māori preference for home birth (Dixon, Prileszky, Guilliland, Miller, \& Anderson, 2014), which would create labour records outside of the NDHB system and would alter these proportions. However, the numbers of homebirths were not likely to be great enough to account fully for the difference. Duration of ROM to birth was compared by ethnicity but no significant differences were found, and it was not included in reporting. Duration from ROM to birth was shorter for primiparas, who were also more numerous in the PROM sample than multiparas. Induction of labour occurred in only $20.1 \%$ of primiparas, versus $35.7 \%$ of multiparas ( $p=0.68)$, and it is unknown why primiparas had shorter mean duration of ROM to birth. 


\subsection{Antenatal Care}

Diagnostic urinalysis was not completed for $61 \%$ of women in the PROM sample during pregnancy. The Primary Maternity Notice does not specify what tests should be performed during pregnancy, or the timing of those tests, however does require LMCs to refer for 'appropriate' screening tests (MOH, 2007). The NZCOM (2016) consensus statement for laboratory testing and screening also directs midwives to collect 'appropriate' laboratory tests, but does not define them, and referenced a laboratory test list that was unable to be located in an internet search. The $\mathrm{MOH}$ webpages for pregnant women state that urinalysis may be done in each trimester, with the purpose of identifying bacteriuria, urinary tract infections, proteinuria and glycosuria ( $\mathrm{MOH}, 2020 \mathrm{a})$. Routine urinalysis is supported by the New Zealand and Australian-produced textbook "Midwifery: Preparation for Practice", which nonetheless states that there is no consensus as to what constitutes best practice in antenatal urinalysis screening (Grigg, 2008). The New Zealand risk-based GBS management strategy states that women with GBS bacteriuria during pregnancy should be offered IAP (Darlow et al., 2015).

Whether routine urinalysis during pregnancy is expected and a forms a foundation of 'riskbased' GBS management is a matter for debate. GBS colonisation of the urinary tract during pregnancy can represent heavy maternal colonisation, with increased risks of preterm labour, pyelonephritis and low birth weight, however recent research has found GBS bacteriuria in pregnancy to have low positive predictive values for vaginal colonisation at birth (Khalil, Thorsen, Møller, \& Uldbjerg, 2018). A recent Cochrane review found the evidence for risks of GBS bacteriuria to be of low quality, and called for research to conduct risk: benefit analyses, including cost-benefit, long term outcomes including the neonatal microbiome, particularly in low-risk women (Schafer \& Phillippi, 2020; Smaill \& Vazquez, 2019).

The absence of urinalysis positive for GBS does not rule out maternal vaginal or gastrointestinal colonisation of GBS. The minimal numbers of women given appropriate urinalysis and/or swab-based screening made it difficult to be accurate when considering risk. The New Zealand Consensus Statement (Darlow et al., 2015) on risk-based GBS management does not require any swabs to be collected; however, some practitioners do 
offer swabs and three women had them collected. Only women with test-specific swabbased screening, or with their ROM event prior to 37 weeks were of known intrapartum GBS risk in this data collection. Swabs may be also collected antenatally to check for chlamydia, gonorrhoea, bacterial vaginosis, candida albicans and trichomonas (Labtests, 2018). It is of some concern that $61 \%$ of women appear to have had no urological screening, and $73.2 \%$ of women appeared to have no sexual health screening in pregnancy.

It is unclear from the data why there were variations in antenatal screening based on ethnicity. The higher proportions of wāhine Māori receiving urinalysis and vaginal swabs may go hand in hand with the higher numbers of wāhine Māori who were given IAP. This raises the question of whether the caregivers for wāhine Māori provided more complete care, or perceived a greater need to test and treat wāhine Māori for communicative diseases.

There was no difference in documented engagement with care based on Māori vs nonMāori ethnicity $(p=0.679)$. Wāhine Māori were more likely to live over $50 \mathrm{kms}$ away from Whangarei Base Hospital, however antenatal care is available throughout the region (New Zealand College of Midwives, 2020). Antenatal diagnostic screening and ultrasound services are available at the main centres of Whangarei, Paihia, Kerikeri and Kaikohe, or in-house at NDHB facilities including Kaitaia Hospital (Northland District Health Board, 2019; Northland Pathology, 2020). Unlike the local availability of primary care, secondary care is only available at Whangarei Base Hospital, and most women birthed there. This pattern of birthing at the secondary hospital is also seen in the NDHB sample, and likely represents a mix of causes and clinical situations.

\subsection{Intrapartum Care}

During intrapartum care there were several components of care that were problematic. These included maternal engagement with care, evidence of diagnosis of SROM, lack of obstetric consultation, longer duration of established labour, assessment of maternal and fetal wellbeing and the use of digital vaginal examinations to assess progress in labour.

It appeared that many women $(40.7 \%)$ in the PROM sample did not engage with any care prior to labour, or if they did, it was not reported in NDHB clinical notes with subsequent 
documentation in the NDHB system. Lack of documented engagement with care was associated with missed obstetric consultation, which is recommended once 24 hours has elapsed without birth having occurred (Bailey, 2015). It is unknown if these women had their needs met, but improved documentation skills could help clarify what care has been provided.

Evidence of diagnosis of SROM was questionable in some cases. Evidence of visible liquor draining was not documented for 57 (46.4\%) women, yet only 27 (22.0\% of total) were examined by speculum. This raises the question of whether SROM was accurately diagnosed - though it is difficult to tell solely from clinical notes. Inaccurate diagnosis may have been associated with the high rate of ARM needed to induce or augment labour (19.5\%). Of the 24 women given an ARM, only three had presented with visible liquor draining, and eight were examined with a speculum. Possibly women who were given an ARM had a 'hind-water leak'27 - which is a recurring topic on pregnancy websites, but largely unexplored when it has occurred at term in literature. The number of ARMs given in the PROM sample would suggest it is a common but perplexing occurrence and reinforces the need for accurate diagnosis during the initial consultation.

Use of vaginal examination to diagnose SROM in ten women meant that they should not have been regarded as eligible for expectant management from that time onwards (Bailey, 2015; Marowitz \& Jordan, 2007), although only one woman had her labour induced subsequent to the vaginal examination. The numbers are insufficient to demonstrate any variation of risk for these women, but this practice is of concern due to introducing risk of maternal infection.

Duration of established labour was increased for both primiparous and multiparous women and augmentation was used more frequently than in women without PROM. Both of these factors support the idea that PROM is symptomatic of pathological disturbance in the labour process, as proposed by Li et al. (2011); and Namli Kalem et al. (2017). Use of pain relief was significantly higher, with $94.3 \%$ of women using some form of documented pain relief

\footnotetext{
${ }^{27}$ Thought to occur when the membranes rupture behind the fetal head, leading to a slow or intermittent loss of amniotic fluid and leaving the forewaters intact.
} 
medications or techniques, including $35.8 \%$ using epidural and $30.9 \%$ using morphine. This is likely to be related to meeting the needs of women given oxytocin infusions for induction and/or augmentation of labour, but possibly may be related to longer duration of labour, fetal malposition or maternal exhaustion (Simkin, 2010; Sinha, Talaulikar, \& Arulkumaran, 2018), both of which were difficult to reliably discern from the clinical notes.

Maternal wellbeing variables included use of IAP, highest maternal temperature in labour, documented suspicion of chorioamnionitis and number of vaginal examinations. IAP was recommended for use in all women as PROM had exceeded 18 hours (Bailey, 2018), but 18 women (14.6\%) did not receive it with no reason for omission documented. Not receiving IAP was associated with suspected chorioamnionitis $(p<0.05)$. Maternal pyrexia raised the clinical suspicion of chorioamnionitis. The incidence of suspected chorioamnionitis was at the higher end of that found in most studies included in the literature review, but well below the $40 \%$ claimed by Ismail and Lahiri (2013). Four of the women with suspected chorioamnionitis were given amoxycillin-clavulanate to treat their symptoms.

Meconium liquor was recorded as a measure of fetal wellbeing, but was only present in 17 (13.8\%) labours and was not considered to be excessive; Lee et al. (2011) found meconium liquor to be a feature of $18.4 \%$ term pregnancies and unrelated to PROM. However, $35.3 \%$ of the babies in the PROM sample who had meconium liquor were subsequently resuscitated at birth $(p<0.05)$, and $29.4 \%$ of babies were admitted to SCBU $(p=0.087)$. This is sufficient proportions to warn caregivers of increased potential that neonatal resuscitation may be needed, and to seek paediatric consultation when meconium liquor is present during PROM.

It is reasonable to assume in many cases that a vaginal examination would be done to assess the need for induction or augmentation of labour, but NDHB policy states that from the time of the first vaginal examination, expectant management should no longer be used (Bailey, 2015). Further, if the woman is not in labour, that an obstetric consultation is recommended (Bailey, 2018). Of the 78 women who had their first vaginal examination before being given IAP (one outlier of $>4$ days excluded) the mean duration from examination to IAP of six hours \pm SD 6:19 is concerning. There were also a high number of vaginal examinations for some women, with $39.8 \%$ of women having between five to 
thirteen examinations. Seaward et al. (1998) found a correlation between seven to eight vaginal examinations and chorioamnionitis $(p=0.04)$. The number of examinations that are beneficial without causing harm varies individually (Dixon \& Foureur, 2010).

\subsection{Labour and Birth Outcomes}

PROM itself affected labour and birth outcomes, particularly mode of birth and the incidence of postpartum haemorrhage. Women in the PROM sample had a lower incidence of vaginal birth (59.3\%) and increased instrumental (13.8\%) and caesarean births (26.8\%), compared to both the NDHB and COMCORD samples. A link between PROM and increased risk of caesarean birth has been considered by many studies, with mixed results. The Cochrane review by Middleton et al. (2017) did not find PROM to be predictive of caesarean birth, and nor did the Hannah et al. (1996) TERMPROM study (included in the Cochrane review). It is worth noting that the incidence of caesarean birth found in the Hannah, et al. study ranged in the four treatment arms from $9.6 \%$ to $10.9 \%$ - well below the $26.8 \%$ found in the PROM sample. Caesarean section rates have almost doubled in since the 1990s in New Zealand and internationally (Betrán et al., 2016). It is probable that were the TERMPROM study repeated today, the findings would differ in this outcome, if not in others

Postpartum haemorrhage occurred almost twice as often in the PROM sample (32.5\%), compared to both the NDHB (17.0\%) and COMCORD (17.2\%) samples. Use of oxytocin in labour increased the incidence of any postpartum haemorrhage $(500 \mathrm{mls}-999 \mathrm{mls}$ combined with $\geq 1000 \mathrm{mls}$ ) to $42.1 \%$, (OR $2.582,95 \% \mathrm{Cl}[1.171,5.693])$. Improvements in the accuracy and consistency in documentation of postpartum blood loss within NDHB would assist in providing confidence in these figures. The literature review process turned up few studies which had included postpartum haemorrhage as an outcome, however there appears to be some evidence to support the finding of this study that PROM increases the risk of postpartum haemorrhage (Middleton et al., 2017; Tran et al., 2008; Xia et al., 2015).

It was challenging to compare perineal trauma, as NDHB had no data recorded for $50 \%$ of their sample. There was an extremely low proportion of women in the NDHB sample with intact perineum recorded, which suggests that field was commonly left blank for women 
who had no perineal trauma. This non-recording skews the NDHB outcomes towards second degree tears, and the data is too problematic to make a useful comparison.

\subsection{Neonatal Outcomes}

Several neonatal outcome measures showed significantly different findings, including birthweight centiles for babies born to women who were smokers versus those who were non-smokers, a high incidence of neonatal resuscitation, and high admissions to SCBU, particularly for Māori pēpi.

The mean birthweight centiles were below average for both the PROM and NDHB samples, with those for Māori pēpi lower still in both samples. Maternal smoking was more prevalent within the PROM and NDHB samples compared to COMCORD, and strongly associated with Māori ethnicity, reflecting a societal issue present in Northland. Smoking itself did not appear to have been associated with PROM, as opposed to the findings of Middleton et al. (2017), but smoking was associated with a high incidence of small for gestational age babies within NDHB $(p<0.01)$, similar to findings by Reeves and Bernstein (2008). The small for gestational age classification of birthweight centiles below the $10^{\text {th }}$ centile (Clausson, Gardosi, Francis, \& Cnattingius, 2001), was applied to $12.1 \%$ of babies in the PROM sample and $16.2 \%$ of NDHB babies. Babies of women who smoke have a greater risk of fetal growth restriction, and babies who are small for gestational age have an increased risk of perinatal morbidity (McCowan, Cowan, \& Stein de-Laat, 2019). NDHB is very aware of the high number of small for gestational age babies, and have made good efforts to provide antenatal wānanga ${ }^{28}$ with a focus on maternal wellbeing and smoking cessation support, as well as a Smoke Free programme with a focus on Māori women. In the $2018 / 19$ year $95 \%$ of women who were smokers in pregnancy were given information and offered support to quit smoking (Northland District Health Board, 2018a, 2019). Management of fetal growth will be returned to later in this chapter.

${ }^{28}$ A meeting or gathering to discuss a topic (Moorfield, n.d.) 
Resuscitation in the PROM sample (16.9\%) was more common than in COMCORD sample (12.8\%) and both are high. The Australian and New Zealand Resuscitation Council (2017) state that $85 \%$ of babies need no actions to initiate breathing within the first 30 seconds of birth, while $10 \%$ will commence spontaneous respirations following drying with a towel and stimulation. Resuscitation is needed by $5 \%$ of babies: by positive airway pressure for $3 \% ; 2 \%$ need intubation; and $0.1 \%$ need chest compressions and/or epinephrine. Resuscitation (16.9\%) was associated with SCBU admissions in the PROM sample $(15.3 \%, p<0.01)$, and both signify an over-representation that is of concern.

NDHB admissions to SCBU were 9.3\% and in 2016, NDHB had the highest documented rate of term babies needing respiratory support in New Zealand, at 4.5\% (Figure 5-1). These rates are modestly affected by the number of admissions subsequent to expectant management of PROM as per the NDHB PROM policy. NDHB commented in their 2018 Maternity Quality and Safety Annual Report that SCBU admissions are an area that the DHB is keen to improve upon. Similarly, they are aware of over-representation in perinatal mortality statistics and have introduced several measures to reduce risks of mortality. These include exploring reasons for late registration with LMCs, fetal movements education for women, increased availability of ultrasound scans, pregnancy risk screening pathways, introduction of GROW fetal growth monitoring, review of perinatal deaths at morbidity and mortality meetings, and exploration of transport issues (NDHB, 2018a). 


\section{Indicator 20: Babies born at $37+$ weeks' gestation requiring respiratory support, 2016}

Figure 35: Percentage of babies born at 37+ weeks' gestation requiring respiratory support, by DHB of residence, 2016

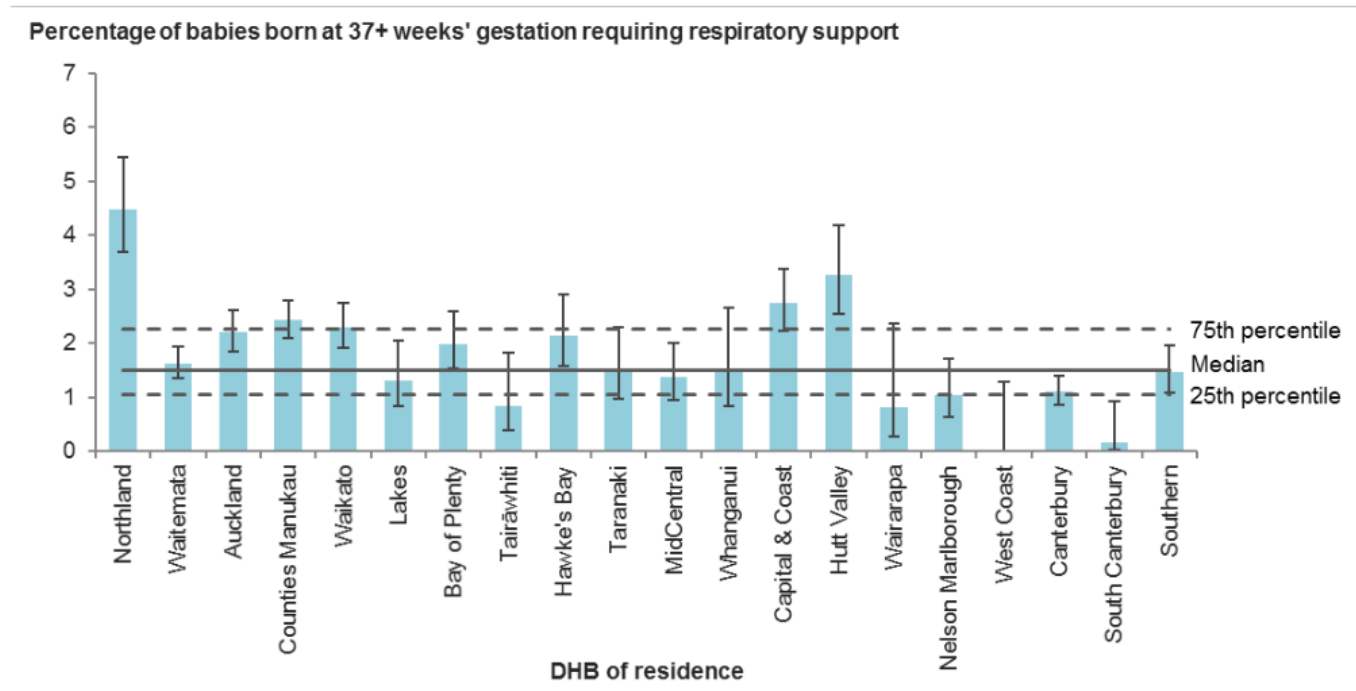

Solid line represents the median percentage of DHBs; dashed lines represent the 25th and 75th percentiles.

Error bars represent $95 \%$ confidence intervals.

Figure 5-1 Clinical Indicator 20 (Ministry of Health, 2018, p.79)

Intravenous antibiotics were given to 14 of the 19 neonates admitted to SCBU, $11.3 \%$ of the total PROM sample neonates, though no babies had sepsis confirmed by culture. The speed of disease progression in neonatal sepsis is sufficiently rapid that antibiotics are typically given to at-risk babies as a preventative rather than as a treatment (Melin, 2011). Recent dialogue has been raised about the phenomenon of culture-negative sepsis, which causes over-medication of neonates with unnecessary antibiotics to the order of six to 16 more times as clinicians attempt to treat apparent symptoms (Klingenberg, Kornelisse, Buonocore, Maier, \& Stocker, 2018). Short and long-term harms of neonatal antibiotics include increasing antimicrobial resistance, necrotising enterocolitis, overgrowth of candida, and death (Cantey \& Baird, 2017; Zea-Vera \& Ochoa, 2015). Collection of adequate blood culture samples, and following international definitions of neonatal sepsis may assist in reducing inappropriate antibiotic use (Klingenberg et al., 2018). An option that may be of use is the Neonatal Early-Onset Sepsis Calculator, an online clinical support tool created by Kaiser Permanente (2020), which has been shown to safely reduce both the number of investigations performed and administration of antibiotics to neonates (Achten et al., 2020; 
Strunk et al., 2018). Another tool that will be useful if not currently utilised is a neonatal early-warning score system to identify deteriorating babies, which is being rolled out across New Zealand through the Neonatal Encephalopathy Taskforce project (Accident Compensation Corporation, 2020).

The low rate of exclusive breastfeeding on discharge (86.3\%) may, for the remaining $13.7 \%$ of babies, reflect a range of factors which may have reduced their ability to exclusively breastfeed - though numbers were too low to establish significance. Mechanical factors such as pressures on the fetal cranial nerves and jaw due to operative births may interrupt nerve signalling and reflexes or cause bruising, inhibiting a neonate's ability to feed successfully (Smith, 2007). Maternal use of opiates (morphine) and epidural pain relief can inhibit neonatal feeding reflexes and reduce maternal engagement with their newborn (Smith, 2007). This was seen in the PROM sample with partial or artificial feeding status on discharge applied to $18.5 \%$ of babies born to women given morphine, $15.8 \%$ after epidural, and $17.6 \%$ after a spinal. Maternal-infant separation through admission to SCBU can also be a factor in reducing exclusive breastfeeding rates (Smith, 2007), which was seen in the PROM sample as $31.6 \%$ of babies admitted to SCBU were partially breastfed on discharge. Effects of care given while in hospital are also a major factor in long-term breastfeeding babies who are fed something other than breastmilk while in hospital are less likely to be breastfed at six weeks of age (Patterson, Keuler, \& Olson, 2019). All NDHB maternity facilities are baby-friendly (Northland District Health Board, 2020), though it is unclear whether the SCBU holds that status.

\subsection{Key Findings}

The purpose of the Key Findings section was to identify whether any specific aspects of care affected the outcomes for the women in each respective group, and to identify factors which could affect the safety and equity of the women and babies in this sample. The factors investigated including Māori vs non-Māori ethnicity, maternal smoking, maternal engagement with care, duration from ROM, intrapartum oxytocin use, use of artificial rupture of membranes and outcomes for babies who were small for gestational age. Other than ethnicity, the only comparison which displayed meaningful and significant results was the use of oxytocin in labour, which was associated with a reduction in vaginal births, and an 
overall increase in postpartum haemorrhage. The differences based on ethnicity display issues of inequity for wāhine Māori and pēpi, despite a visually engaged DHB (Northland District Health Board, 2018a, 2018b, 2019).

Comparative outcomes for women in the sample based on Māori and non-Māori ethnicity provided an interesting and thought-provoking contrast. Some outcomes appeared beneficial at first glance, such as the low incidence and negative association with epidural use, and the increased and positive association with IAP use. It is a matter for discussion and further research as to whether wāhine Māori have equitable access to epidurals, or if they are targeted for IAP in deficit-based care practices, or feel less agency in decisionmaking in a Eurocentric, secondary maternity environment. The higher rate of vaginal births (with corresponding lower rates of instrumental and caesarean births) is to be celebrated and may be associated with the lower incidence of epidural use. This presents a dilemma: are epidurals used less because labour has progressed well; or has labour progressed well because epidurals have been avoided? In either case, it is likely there are other factors that influence this as well.

Māori pēpi were more likely to be resuscitated, be admitted to SCBU for any reason, have any respiratory diagnosis and to receive antibiotics. The contrast between the positive birth outcomes and the concerningly high rate of morbidity amongst the wāhine Māori and pēpi of the sample suggests a divide between expectations of normality and the reality of poor and inequitable outcomes (Perinatal and Maternal Mortality Review Committee, 2018; Stevenson, Filoche, Cram, \& Lawton, 2020).

Maternal health is not a topic referred to in the WAI 2575 Waitangi Tribunal Health Services and Outcomes Inquiry, however low birthweight, breastfeeding status and mortality of infants are. The Māori Health Trends report states Māori babies are at greater risk of having low birthweight (defined as under $2500 \mathrm{~g}$ ), and attributes this to poor maternal nutritional status and maternal smoking. Māori babies are less likely to be breastfed at three and six months of age than non-Māori babies. Māori infant mortality has improved over the past two decades but remains 1.2 per 1000 live births higher than for non-Māori. Sudden unexpected death in infancy rates have also improved significantly, but remain more than three times higher than for non-Māori (Ministry of Health, 2019c; Waitangi Tribunal, 2019). 
Māori leadership in healthcare, cross-sector action, and equity, quality and safety for Māori are aspects proposed by Whakamaua: Māori Health Action Plan 2020-2025 (Ministry of Health, 2020b), which does not speak to specific health issues, but provides a basis for potential changes to the way Māori healthcare is managed. It is hoped that through these projects that systemic changes are introduced to prioritise Māori health and healthcare provision.

The proportion of midwives who are Māori was 9.83\% in 2019 (Midwifery Council of New Zealand, 2019), but still remains well below the national population of $16.5 \%$ Māori and Northland population of 36.0\% Māori (Stats NZ, 2020). Wāhine Māori made up $45.8 \%$ of the 2018 Northland birthing population (NDHB, 2018a), similar to the $49.3 \%$ found in the NDHB sample. The need for more Māori midwives has been recognised in a statement supporting the growth of the Māori midwifery workforce in the Midwifery Staffing Standards (Midwifery Employment Representation and Advisory Service, 2014). The Ministry of Health has also announced the existence of a strategy to increase numbers, but the details have not yet been made public other than a small 2019 budget allocation to support Māori undergraduate midwifery students (Midwifery Employment Representation and Advisory Service, 2019). The Aotearoa Midwifery Project being undertaken by the Midwifery Council of New Zealand (2020) may also develop strategies to increase the numbers of Māori midwives.

NDHB discusses several initiatives to promote maternity healthcare equity for wāhine Māori. The DHB offers a two-day kaupapa Māori antenatal education wananga which focuses on wellbeing, SUDI prevention, wahakura ${ }^{29}$ weaving, traditional Māori birthing practices and postpartum care. The wananga are run throughout the region and engages with Māori health providers to promote engagement by women, midwives and other healthcare professionals. There is a region-wide SUDI prevention programme. NDHB runs Te Whare Ora Tangata, which are regular hui for government and DHB agencies to collaborate on wrap around care and appropriate referrals for families and whānau who are

${ }^{29}$ A woven basket for a baby to sleep in (Moorfield, n.d.) 
known to need assistance. NDHB are planning additional training for doctors and midwives, using Turanga Kaupapa as a framework for clinical practice (NDHB, 2018a, 2019).

\subsection{A reflection on 'normal birth'}

The final comparison in the Findings chapter contrasted the outcomes for the women in the PROM sample to those in the NDHB sample and presented an eye-opening contrast. It is acknowledged that the NDHB sample represents all other births, not 'normal' births. Compared to the NDHB sample, women in the PROM sample were at significantly greater risk of medicalisation of their births, needed greater pain relief, were more likely to need operative assistance to birth, more likely to haemorrhage or see their baby resuscitated or admitted to SCBU, and were less likely to exclusively breastfeed their baby. In many cases, interventions such as using oxytocin, will have often led to further issues, described as a 'cascade of interventions' by (Thorogood \& Donaldson, 2008). Each of these factors may have long-term effects for women and/or their babies, and taken as a whole, provide a concerning picture of care received by women. The earlier comparisons based on independent factors or actions could not display great differences based on lifestyle or individual actions taken in labour, and it appeared that PROM itself was the major concern, significantly challenging women's ability to birth normally.

In the interest of finding out how many women had a 'normal birth', an exercise to eliminate non-normative factors was undertaken (Figure 5-2). The World Health Organisation (1996) defines normal birth as including spontaneous onset and progress in labour at term, with a spontaneous vertex birth of the baby, and with mother and baby in good condition. For the purposes of this simple exercise, women were eliminated from consideration of having had a 'normal birth' if they:

- Needed induction of labour

- Had oxytocin for augmentation

- Had an instrumental or caesarean birth

- Had pyrexia of 38.0 degrees or more

- Had a postpartum haemorrhage (over $500 \mathrm{mls}$ )

- Their baby needed resuscitation 
- Their baby was admitted to SCBU for respiratory support

Women were not excluded if they had an ARM, as it is a common action within the scope of midwifery care and may be performed in a primary setting. Women were also not excluded if they had used an epidural, as pain relief is a human right - though it is recognised many definitions of normal birth would exclude epidurals.

\section{Normal Birth Flow Diagram}

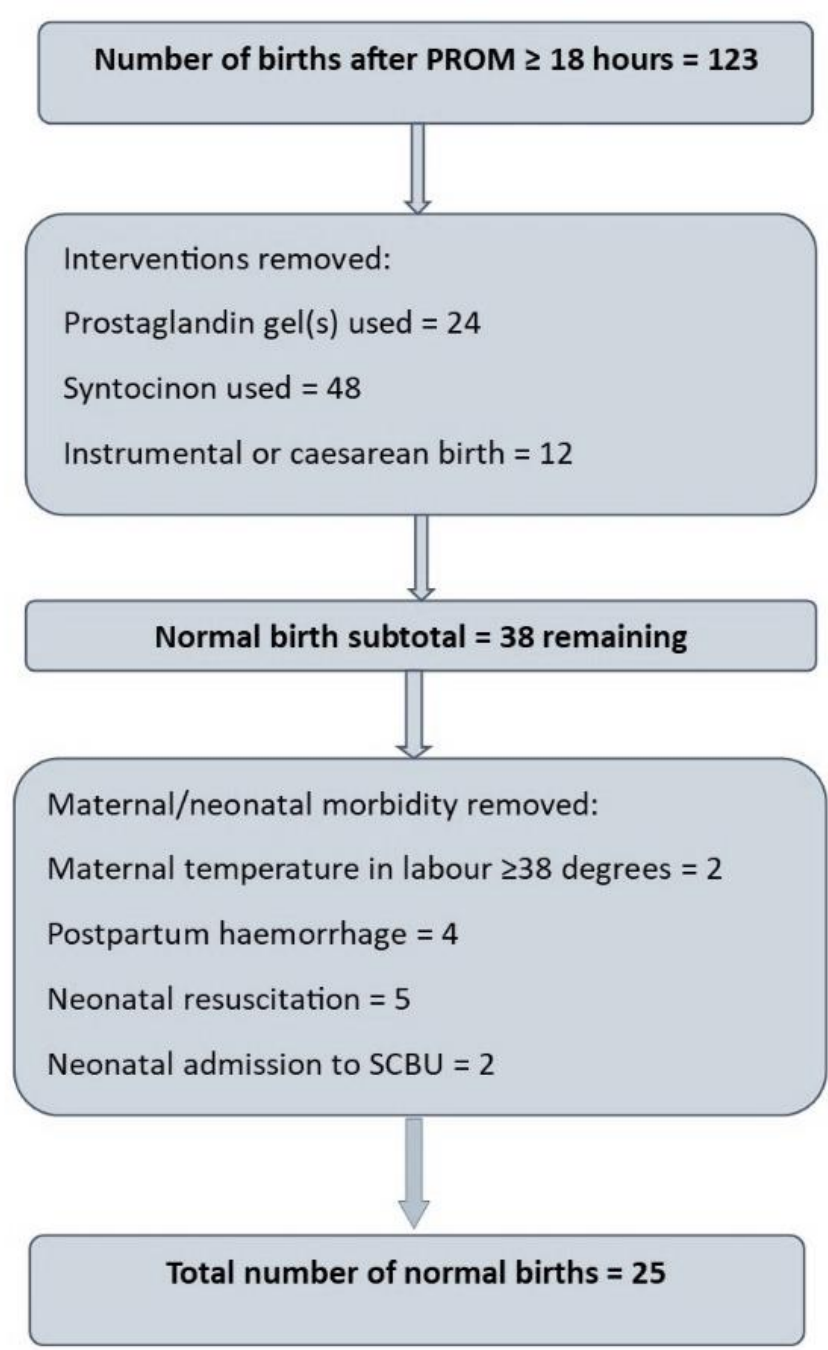

Figure 5-2 Flow diagram of elimination of non-normal birth features

By reducing the field in this manner, firstly through removing interventions including medicated induction or augmentation, instrumental or caesarean birth, only 38 (30.9\%) of women remained. By subsequently removing morbidity outcomes including pyrexia of $\geq 38$ 
degrees, postpartum haemorrhage, neonatal resuscitation, or admission to SCBU, 25 (20.3\%) women remained. There was little variation by ethnicity, as 15 (24.2\%) of European women and 10 (21.3\%) of wāhine Māori had 'normal' births. Just nine (13.4\%) of primiparous women had normal births, compared to $16(28.6 \%)$ of multiparas. There did not appear to be equity issues regarding this definition of normal birth for Māori women in this assessment, but overall the results are concerning, which leads to the question, "what was the benefit of waiting so long, if only $20.3 \%$ of women and babies had normal and safe outcomes?

The benefit of waiting and being less prescriptive about care for this group of women is that ideally, they had time for labour to develop as naturally as possible, and input into their decisions about their care, and that as a group, maternal outcomes did not deteriorate over time, if time was the only factor (though concerns remain about neonatal well-being). Women's right to participate in decision-making is enshrined in New Zealand law (Health and Disability Commissioner, 1996). In a large American study of women's perceptions in decision-making in labour, Attanasio, Kozhimannil, and Kjerulff (2018) found that Black women without university degrees or health insurance, and women having labour induced reported reduced involvement in decision-making. Obstetric procedures undermined women's subsequent perception of their ability to engage and influence decision-making about their care. The findings of this article are of relevance to the New Zealand setting as we endeavour to provide equitable maternity care to wāhine Māori and pēpi, but also for all women who find their labour has become medicalised and may feel their control has been eroded. Women want to feel safe, but not at the expense of all other considerations (Grigg, Tracy, Daellenbach, Kensington, \& Schmied, 2014). There is little current research about PROM which includes maternal satisfaction measures (Middleton et al., 2017), therefore it is currently not possible to conclude whether women would prefer to have ample time for labour to unfold, or to be more pragmatic and induce labour at an earlier point.

Returning to the aims of this study, it has been shown that the NDHB PROM policy was applied in practice, in that 123 women took over 18 hours from ROM to birth, and there were a wide variety of care pathways utilised. In the broad sense it can be argued that the needs of birthing women experiencing PROM were met, by providing adequate and appropriate care, albeit strongly focused at Whangarei Base Hospital, but with heavy 
reliance on active labour management, pain relief, obstetric assistance to birth, and neonatal support. Whether the women were satisfied with their births is a different question that this project can't answer. The current policy appears to be somewhat safe for women as there was little variation in outcomes based on individual actions taken and increasing duration - and women's choices did not tend to impact negatively upon themselves except when recommended care was repeatedly declined. However, PROM itself appeared to greatly increase risks for women and their babies, with increased number of babies requiring resuscitation and/or admission to SCBU, and it would be timely to revise the NDHB PROM policy with regard to current consensus and practice guidelines and the findings of this thesis. All studies have their limitations, however, and in the next section, the limitations of this study will be discussed, followed by recommendations for practice, education and future research.

\subsection{Study limitations}

\subsubsection{NDHB data errors}

During data collection for the PROM sample, errors in data recording were evident when comparing clinical notes to hand-written Labour and Birth records and electronic Maternity Plus data records. Of 157 maternal records reviewed, 66 (42\%) had factual or documentation errors, 51 of which were in Maternity Plus data. In 31 (19.7\%) files the error occurred in both the Labour and Birth Record and Maternity Plus entries, while only four (2.5\%) of files had an error solely in the Labour and Birth Record. 20 files were excluded from final data analysis for this thesis due to finding errors and finding the file no longer met inclusion criteria. The nature of each error was logged in Appendix 7 and this information has been supplied to the NDHB Maternity management team for their reference.

The clinical notes were the ultimate documentary evidence of labour and birth events as they are the primary record and should be completed in real time. The Labour and Birth Record may be completed as events occur or retrospectively following the completion of a birth, and the writer usually refers to the clinical notes to inform their entries. The Labour and Birth Record may have been completed by more than one person, but there was no indication of who makes any entry. Maternity Plus data was entered directly from the 
Labour and Birth Record, typically without reference to the clinical notes; therefore, any errors on the Labour and Birth Record are likely to be repeated in the Maternity Plus data.

Many errors could be sorted into groups:

- Handwritten errors of time or date on the Labour and Birth record occurred in 21 files; and 14 Maternity Plus entries contained '00:00' times unrelated to any event documented in the clinical notes - the entries were there simply to fill the field so Maternity Plus would permit the user to progress.

- Ten files contained errors in documenting the use of morphine, likely due to the Labour and Birth record and Maternity Plus system not having been adjusted to include morphine as a medication.

- An unquantifiable error related to height and weight records held by NDHB for some women. This affected the results generated by the birth centile calculator (Australian Bureau of Statistics, n.d.) and was noticeable in the PROM sample, where a handful of babies were under the tenth centile for birthweight, but had birthweights over $4 \mathrm{~kg}$, making growth restriction improbable. This was a limitation of this retrospective study but may have been improved upon by recording evidence of maternal height and weight for the PROM sample rather than relying on the BMI generated by Maternity Plus in data collection.

- An issue not treated as an error but potentially problematic was that two members of staff consistently noted postpartum blood loss in the clinical notes as either $<$ or $>$ average. In these cases, the number entered in Maternity Plus was used - but it is not possible to know if the values entered into Maternity Plus are correct understandings (typically by an administrator) of what was implied by those symbols.

The data for the PROM sample used for analysis in this thesis has been checked for accuracy and consistency, and data entry into SPSS for analysis was also checked a second time. However, data taken from the NDHB data download has not been checked other than for impossible outliers of gestation, and it is acknowledged that based on the errors found in the 157 files reviewed by hand, there must be an unknown degree of error in the NDHB database material. The NDHB sample was noted to have missing data, such as values for 
perineal trauma, and under-reporting of pain relief; 187 NDHB women birthing by caesarean section had no statistical record of either epidural or anaesthesia. This is of concern but assessing it for accuracy was outside the scope of this review and the data was therefore accepted as given. The COMCORD data was supplied by COMCORD staff after data cleaning, however some obvious errors remained present, e.g. impossible values for time parameters. Following consultation with COMCORD staff these values have been removed from the data. It is unknown if there are other errors that are not visible due to being plausible, and again it must be otherwise accepted as given.

\subsubsection{General limitations}

It was an intention of this study to record who was engaged in making decisions, particularly around whether to use expectant management or induce labour. Once presented with the clinical notes, it was quickly apparent that it was impossible to keep track of who made decisions, what information was given, and whether the woman was involved and to what degree. It was also hoped to record fetal position in labour, but this was very commonly not documented in the clinical notes. A prospective study which specifically seeks this information may have more success.

There were several data items that would have been good to collect but were omitted: the purpose of each group of blood tests performed (i.e. booking bloods, second antenatal, glucose tolerance test); transfers of women in established labour; and cervical dilatation at first vaginal examination would all have been useful variables to have collected.

A learning from this process has been to prepare for data collection by seeking data definitions that are already in use, such as ethnicity codes or postpartum haemorrhage ranges, so that data doesn't need to be recoded in order to make comparisons with external databases. This would support validity, though double checks were made to ensure transposed data was correct.

\subsection{Significance of the study}

This evaluation study is significant as it considers whether the NDHB PROM policy is applied locally in practice and reflects whether this policy meets the needs of women and babies. It 
is also significant in considering whether the current policy is safe for NDHB practitioners, women, and babies, and reflects whether the application of this policy contributes to the high rate of neonatal admissions to SCBU. This study contributes to the dialogue as to whether it is better to promote active or expectant management following PROM.

\subsection{Recommendations for practice, education, and research}

\subsubsection{Practice and education}

The recommendations arising from this study include location-specific actions as well as broader practice points for all midwives and clinicians, and a comment on future research. Before delving into these comments, it is worth noting that the Health and Disability System Review (2020) recommended reducing of the number of DHBs from 20 to between eight to 12 within the next five years, under which it is possible NDHB could be merged with Waitemata DHB, or Auckland DHB could potentially absorb both. A merger of this kind would present multiple organisational challenges, not least of which would be managing policy differences.

That said, it is worth revisiting the NDHB PROM policy, which was published in 2015 and is due for review. Most of the literature referred to by this policy has been updated, and newer recommendations may be of use. A Cochrane review which was ambivalent between expectant management versus planned early birth (Dare et al., 2006) has been revisited, and the more recent review supports planned early birth, but comments that modern and longitudinal research is needed to identify the benefits versus risks of both management options (Middleton et al., 2017).

A NICE guideline has been updated and provides a care pathway for women not in labour within 24 hours, and includes recommendations for actions not currently in use by NDHB including auscultation of the fetal heart every 24 hours, and for women to birth in a facility with neonatal services, and to remain inpatient for a minimum of 12 hours postpartum (NICE, 2014). The remaining two publications are the RANZCOG College Statement regarding term PROM, updated in 2017; and the EOGBS technical report by the New Zealand GBS Consensus Working Party published in 2004, also updated as the New Zealand 
Consensus Statement (Darlow et al., 2015). Revision of the NDHB PROM policy will need to update these references, as well as consider some of the newer recommendations, particularly in the light of the high number of neonates admitted to SCBU following maternal PROM.

In reflecting on the NDHB PROM policy, it is worth remembering the geographic and demographic conditions within which NDHB operates. Deprivation and distances from services can be high, and the numbers of term babies born small for gestational age and those needing admission to SCBU are also higher than national averages (Ministry of Health, 2019b). NDHB must balance the optimal delivery of services within their means, and within what the population wants and needs. The NDHB policy provides for induction of labour either immediately if indicated, the following morning if not immediately indicated, or for expectant management of up to 96 hours. This provision doesn't differ by reducing options, but provides for women and clinicians to make appropriate informed decisions for care (Health and Disability Commissioner, 1996) within a timeframe that has not been shown to be problematic in the broader sense by Hannah et al. (1996). However, face-to-face antenatal clinical assessment of mother and foetus every 24 hours (with documentation, shared to NDHB records), and encouraging women to birth at Whangarei Base Hospital, particularly if poor fetal growth is a factor, would both be sensible additions to the PROM policy. Reminders to LMCs of the need for obstetric consultation at 24 hours, and documentation of that consultation would be beneficial.

Antenatal screening education for LMCs may be of use, to promote understanding of the purpose of blood tests, urinalysis, and sexual health screening. If not already a part of antenatal class programmes, including the two-day antenatal wananga for women, this would be a great topic to include so that women expect and want to complete these tests. Consideration could be given to reviewing the NZCOM consensus statements and practice guidance around antenatal screening in the Handbook for Practice (New Zealand College of Midwives, 2015).

Accurate fundal height measurement as described by the Growth Assessment Programme (GAP), combined with Gestation Related Optimum Weight (GROW) charts customised to individual women are a useful tool which can increase in identifying which babies are small 
for gestational age, and should be used in every pregnancy (McCowan et al., 2019). In response the Neonatal Encephalopathy Taskforce project (Accident Compensation Corporation, 2020), NDHB has recently begun encouraging all LMCs to participate in GAP training through a local instructor and via Zoom, though the midwifery educator notes it may take some time to alter existing practice (W. Taylor, personal communication, July 14, 2020). Another useful tool is the Small for Gestational Age Risk Assessment Tool developed by J. Cowan, L. McCowan and L. Sadler and published in McCowan et al. (2019), which provides risk factors for small for gestational age babies and suggests care pathways to help identify and manage pregnancies with fetal growth retardation.

Another point for improvement would be to offer education or professional support as to how to accurately diagnose PROM using a speculum as needed, and the boundaries of appropriate use of digital vaginal examinations in the interest of reducing the risk of infection. Ten women (8.1\%) were given digital vaginal examinations and were theoretically ineligible for expectant management, though it continued to be used and this created unnecessary risk for those women and their babies. It may be possible for NDHB to offer Amnisure (Qiagen Sciences, 2019) for use within their facilities, which would provide clarity in circumstances where history may be unclear and liquor not clearly visible on examination. Amnisure bears a cost, however reducing the numbers of women having labour induced with incorrectly diagnosed ruptured membranes, with all of the subsequent increased risks and costs is likely to reduce overall costs and reduce transfers from primary centres that don't need to occur. The findings of this thesis would also recommend minimising the duration from the first vaginal examination to IAP to reduce maternal pyrexia and neonatal morbidity.

Documentation of events was problematic at both an organisational and individual level, and education may be of benefit for LMCs and core staff. The current lack of interface between DHB and midwifery-held notes undermines interprofessional communication. When primary caregivers refer patients into a secondary service there is a written referral which provides a history, however when a woman is accompanied by her LMC as part of labour care, this process is omitted. There is also often not a full assessment completed by a receiving staff member, because clinical responsibility typically remains with the LMC. The content of documentation education needs to cover recording the process of informed 
decision-making, and documenting a summary of care when the woman enters a facility and moves into a new clinical records system. A secondary benefit of this education would be to improve data recording within Maternity Plus, which would provide both a more accurate record for each individual woman, as well as more reliable statistics. Aligning data collection reference ranges to those used in national data collection, for e.g. blood loss increments would improve the comparability of outcomes. An alternative that would resolve both these issues would be to use one consistent maternity information system throughout New Zealand, which would be of benefit to women, LMCs, maternity services and taxpayers.

The web-based Neonatal Sepsis Calculator produced by Kaiser Permanente (2020) may be of use to assist with care planning for individual babies in the SCBU setting, but it is a tool that can be used by any maternity caregiver to assess and anticipate the level of risk for any given neonate, and it provides recommendations for testing and treatment as indicated by the clinical picture. The calculator is currently in use at Christchurch Women's Hospital (Canterbury District Health Board, 2020). Another tool that will be useful if not currently utilised is a neonatal early-warning score system to identify deteriorating babies, which is being rolled out across New Zealand through the Neonatal Encephalopathy Taskforce project (Accident Compensation Corporation, 2020).

\subsubsection{Future research}

Future research focusing on PROM is likely to be deferred by researchers and funders until rapid identification of GBS has been comprehensively trialled, proven cost-effective, and management of GBS in labour has changed as a result. It is probable that most women given IAP are GBS negative and have no need for the IAP they are given. Maternal intrapartum antibiotic use has been associated with changes in the neonatal gut microbiome, of which the long term health effects are the subject of ongoing research interest (Azad et al., 2016; Tanaka et al., 2009). There are also concerns about maternal allergic reactions to antibiotics, and increasing maternal and neonatal antibiotic resistance (Ohlsson \& Shah, 2014). 
Studies have compared methods of GBS testing (Brigtsen et al., 2015), vaginal and rectal swab sites (Carrillo-Ávila, Gutiérrez-Fernández, González-Espín, García-Triviño, \& GiménezLirola, 2018), and over the past decade population-based implementation of universal GBS screening in labour using rapid-PCR for GBS (El-Helali et al., 2012; El-Helali et al., 2019; Helmig \& Gertsen, 2019). Two large studies are in process in the United Kingdom: GBS2 and GBS3. GBS2 is pre-publication and explored the accuracy of the Cepheid GeneXpert ${ }^{\circledR}$ (Cepheid, n.d.) GBS rapid-PCR swab test system and whether it reduced maternal and neonatal antibiotic usage (Birmingham Clinical Trials Unit, 2020). GBS3 plans 320,000 participants across 80 UK hospitals, and will be a prospective study looking at the benefit of routine, universal intrapartum screening for GBS, versus existing risk-based management.

GeneXpert ${ }^{\circledR}$ results are available within one hour of collecting a low vaginal and perianal swab when women present in labour, with sensitivity of $93.1 \%$ and specificity of $96.67 \%$ (Ramesh Babu et al., 2018). GBS3 was planned to commence in April 2020, but has been postponed until the COVID-19 crisis has eased (Nottingham Clinical Trials Unit, 2020). A study of this size and quality stands to change UK practice from risk-based to universal screening, which may have a knock-on effect to Australia and New Zealand.

Universal screening using rapid-PCR testing for GBS has been shown to improve antibiotic stewardship, as IAP is consequently only given to women who are currently GBS positive (Fullston, Doyle, Higgins, \& Knowles, 2019; Seedat et al., 2019). Giving IAP to the appropriate recipients has been shown to be safe (Helmig \& Gertsen, 2019) and reduce overall hospital costs (El-Helali et al., 2019).

PROM management could then be revisited, as the ability to eliminate or identify the risks of GBS carriage and transmission may alter the perceived urgency of needing to achieve birth within a specific timeframe after ROM - though GBS positive women are likely to be recommended to accept active management. A further benefit of reducing or redirecting IAP use is to protect the maternal-neonatal microbiome (Azad et al., 2016; Gonzalez-Perez et al., 2016; Mueller, Bakacs, Combellick, Grigoryan, \& Dominguez-Bello, 2015).

In the meantime, the status-quo stands in New Zealand, and it would now be poor fiscal management to fund a study about PROM that failed to include rapid-PCR. A prospective study has been proposed within the New Zealand On Track Network to explore the 
feasibility of using rapid-PCR in labour within New Zealand hospitals (On Track Network, 2020), and this may be the introduction of a practice change in New Zealand.

Future research following on from the feasibility and adoption of rapid-PCR testing into PROM management should focus on the safety and acceptability of active versus expectant management in large-scale prospective studies which are powered to achieve statistical significance in findings, in the present day environment of maternity care at that time. It isn't currently known whether New Zealand maternity units are actually offering 'planned early birth' as an option for low risk women as recommended by Middleton et al. (2017), or if this is only offered conditionally, based on clinician preference and facility acuity.

It is apparent from each of the PROM, NDHB and COMCORD samples that recent research has not reflected the realities of present-day practice in New Zealand well, from perspectives of good clinical practice, the outcomes experienced by women, and maternal engagement in decision-making. Maternal satisfaction is also a measure that has been almost comprehensively ignored. It is no longer acceptable to women that care be imposed or withheld based on the outcomes, opinions and experiences of an earlier generation or two - who were reported to have disliked one of the options a little less than the others (Hannah et al., 1996). Future research must be conducted with reference to informed consent processes and explore planned early birth and expectant management in the New Zealand maternity system.

\section{Conclusion}

PROM at term significantly increased the risks for the birthing women and their babies in this study, and healthcare inequities for Māori were presented and explored. Decisionmaking during the intrapartum period appears to have been within the terms of the NDHB PROM policy, and to have not impacted negatively on labour and birth outcomes, even when the duration from rupture of membranes to birth was excessively prolonged by the standards of national recommendations. The NDHB PROM policy references outdated material and is due for revision, and the findings of this thesis may be constructive to that process. 
Discussion

The quality of clinical documentation was problematic, and there are several recommendations for clinical education to assist with improving outcomes for women affected by PROM, as well as generally within the Northland region. Future research to better manage PROM is needed and should consider maternal advice and satisfaction but is likely to be postponed until rapid GBS testing in labour has been trialled and incorporated into standard care. Accurate diagnosis of GBS status will improve antibiotic stewardship and allow intrapartum care to focus on what care pathways women want and benefit most from, when faced with this problematic clinical issue. 


\section{References}

Accident Compensation Corporation. (2020). Supporting Safer Treatment. Retrieved from https://www.acc.co.nz/for-providers/treatment-safety/

Achten, N. B., Visser, D. H., Tromp, E., Groot, W., van Goudoever, J. B., \& Plötz, F. B. (2020). Early onset sepsis calculator implementation is associated with reduced healthcare utilization and financial costs in late preterm and term newborns. European Journal of Pediatrics, 179(5), 727-734. doi:10.1007/s00431-019-03510-9

Agnes, J. M. B., \& Lavanya, S. (2018). Two-year comparative study on immedicate versus delayed induction in term premature rupture of membranes. International Journal of Reproduction, Contraception, Obstetrics and Gynaecology, 7(1), 94-98. doi:http://dx.doi.org/10.18203/2320-1770.ijrcog20175529

American College of Obstetricians and Gynecologists. (2020). ACOG committee opinion: Prevention of group $B$ streptococcal early-onset disease in newborns.: American College of Obstetricians and Gynecologists. Retrieved from https://www.acog.org/clinical/clinical-guidance/committeeopinion/articles/2020/02/prevention-of-group-b-streptococcal-early-onset-diseasein-newborns

Ashmore, S., Ruthven, T., \& Hazelwood, L. (2011a). Stage 1: Preparation, planning and organisation of clinical audit. In R. Burgess (Ed.), New principles of best practice in clinical audit. (pp. 23-58). Oxon, United Kingdom: Radcliffe Publishing Ltd.

Ashmore, S., Ruthven, T., \& Hazelwood, L. (2011b). Stage 2: Measuring performance. In R. Burgess (Ed.), New principles of best practice in clinical audit (pp. 59-80). Oxon, United Kingdom: Radcliffe Publishing Ltd.

Ashmore, S., Ruthven, T., \& Hazelwood, L. (2011c). Stage 4: Sustaining Improvement. In R. Burgess (Ed.), New principles of best practice in clinical audit (pp. 93-106). Oxon, United Kingdom: Radcliffe Publishing Ltd.

Ashwal, E., Krispin, E., Aviram, A., Aleyraz, E., Gabby-Benziv, R., Wiznitzer, A., . . . Hiersch, L. (2016). Perinatal outcome in women with prolonged premature rupture of membranes at term undergoing labor induction. Archives of Gynecology \& Obstetrics, 294(5), 1125-1131. doi:10.1007/s00404-016-4126-6

Attanasio, L. B., Kozhimannil, K. B., \& Kjerulff, K. H. (2018). Factors influencing women's perceptions of shared decision making during labor and delivery: Results from a largescale cohort study of first childbirth. Patient Education and Counseling, 101(6), 11301136. doi:https://doi.org/10.1016/i.pec.2018.01.002

Australian and New Zealand Resuscitation Council. (2017). ANZCOR Guideline 13.1 Introduction to resuscitation of the newborn infant. Retrieved from https://www.nzrc.org.nz/assets/Guidelines/Neonatal-Resus/ANZCOR-Guideline13.1-June2017.pdf

Australian Bureau of Statistics. (n.d.). Sample Size Calculator. Retrieved from http://www.nss.gov.au/nss/home.nsf/pages/Sample+size+calculator

Ayaz, A., Saeed, S., Usman Farooq, M., Ahmad, F., Ali Bahoo, L., \& Ahmad, I. (2008). Pre-labor Rupture of Membranes at Term in Patients with an Unfavorable Cervix: Active versus Conservative Management. Taiwanese Journal of Obstetrics and Gynecology, 47(2), 192-196. doi:https://doi.org/10.1016/S1028-4559(08)60079-0 
Azad, M. B., Konya, T., Persaud, R. R., Guttman, D. S., Chari, R. S., Field, C. J., . . Cyr, M. (2016). Impact of maternal intrapartum antibiotics, method of birth and breastfeeding on gut microbiota during the first year of life: a prospective cohort study. BJOG: An International Journal of Obstetrics \& Gynaecology, 123(6), 983-993. doi:10.1111/1471-0528.13601

Babbie, E. (2008). The Basics of Social Research. (Fourth ed.). Belmont, CA: Thomson Wadsworth.

Baby Friendly Hospital Initiative. (2020). Part 1: Background and Baby Friendly implementation in Aotearoa New Zealand Retrieved from https://www.babyfriendly.org.nz/fileadmin/Documents/1 BFI Resource Document Part One new.pdf

Bailey, D. (2015). Prelabour rupture of membranes at term (PROM). Whangarei, New Zealand: Northland District Health Board

Bailey, D. (2018). Group B Streptococcus - Prevention of early onset neonatal infection. Whangarei, New Zealand: Northland District Health Board.

Barišić, T., Mandić, V., Tomić, V., Zovko, A., Novaković, G., Barišić, T., . . Novaković, G. (2017). Antibiotic prophylaxis for premature rupture of membranes and perinatal outcome. Journal of Maternal-Fetal \& Neonatal Medicine, 30(5), 580-584. doi:10.1080/14767058.2016.1178228

Betrán, A. P., Ye, J., Moller, A.-B., Zhang, J., Gülmezoglu, A. M., \& Torloni, M. R. (2016). The increasing trend in caesarean section rates: Global,regional and national estimates: 1990-2014. PLOSONE, 11, e0148343. doi:10.1371/journal.pone.0148343

Birmingham Clinical Trials Unit. (2020). GBS2: Accuracy of a rapid intrapartum test for maternal group B streptococcal colonisation and its potential to reduce antibiotic usage in mothers with risk factors. Retrieved from https://www.birmingham.ac.uk/research/bctu/trials/portfolio-v/GBS2/index.aspx

Bishop, E. H. (1964). Pelvic scoring for elective induction. Obstetrics \& Gynecology 1964, 24(2), 266-268. Retrieved from https://journals.Iww.com/greenjournal/Citation/1964/08000/Pelvic Scoring for Ele ctive Induction .18.aspx

Bricker, L., Peden, H., Tomlinson, A., Al-Hussaini, T., Idama, T., Candelier, C., . . Alfirevic, Z. (2008). Titrated low-dose vaginal and/or oral misoprostol to induce labour for prelabour membrane rupture: a randomised trial. 115(12), 1503-1511. doi:10.1111/j.1471-0528.2008.01890.x

Brigtsen, A. K., Dedi, L., Melby, K. K., Holberg-Petersen, M., Radtke, A., Lyng, R. V., . . . Whitelaw, A. (2015). Comparison of PCR and serotyping of Group B Streptococcus in pregnant women: The Oslo GBS-study. Journal of Microbiological Methods, 108, 3135. doi:https://doi.org/10.1016/i.mimet.2014.11.001

Brophy, S., Snooks, H., \& Griffiths, L. (2008). Small-Scale Evaluation in Health. doi:10.4135/9781849209892

Bryman, A. (2016). Social Research Methods. Oxford, United Kingdom: Oxford University Press.

Bui, Y. N. (2009). How to write a Master's thesis. Thousand Oaks, CA: Sage Publications, Inc.

Cabrera, I. B., Quiñones, J. N., Durie, D., Rust, J., Smulian, J. C., \& Scorza, W. E. (2016). Use of intracervical balloons and chorioamnionitis in term premature rupture of membranes. Journal of Maternal-Fetal and Neonatal Medicine, 29(6), 967-971. doi:10.3109/14767058.2015.1027191 
Canterbury District Health Board. (2020). Neonatal clinical resources: Christchurch Women's Hospital. Christchurch, New Zealand: Christchurch Women's Hospital Retrieved from https://edu.cdhb.health.nz/Hospitals-Services/Health-Professionals/maternity-careguidelines/Documents/2403289\%20Neonatal\%20Clinical\%20Resource.pdf

Cantey, J. B., \& Baird, S. D. (2017). Ending the Culture of Culture-Negative Sepsis in the Neonatal ICU. Pediatrics, 140(4), e20170044. doi:10.1542/peds.2017-0044

Carrillo-Ávila, J. A., Gutiérrez-Fernández, J., González-Espín, A. I., García-Triviño, E., \& Giménez-Lirola, L. G. (2018). Comparison of qPCR and culture methods for group B Streptococcus colonization detection in pregnant women: evaluation of a new qPCR assay. BMC Infectious Diseases, 18(1), 305. doi:10.1186/s12879-018-3208-4

Centers for Disease Control and Prevention. (2018). Group B Strep (GBS). Retrieved from https://www.cdc.gov/groupbstrep/about/prevention.html

Cepheid. (n.d.). Xpert GBS LB \& Xpert GBS. In. Sunnyvale, CA: Cepheid.

Chan, W. S. W., Chua, S. C., Gidding, H. F., Ramjan, D., Wong, M. Y. W., Olma, T., . . G Gilbert, G. L. (2014). Rapid identification of group B streptococcus carriage by PCR to assist in the management of women with prelabour rupture of membranes in term pregnancy. Australian \& New Zealand Journal of Obstetrics \& Gynaecology, 54(2), 138-145. doi:10.1111/ajo.12145

Chaudhuri, S., Nath Mitra, S., Kumar Banerjee, P., Kumar Biswas, P., \& Bhattacharyya, S. (2011). Comparison of vaginal misoprostol tablets and prostaglandin E2 gel for the induction of labor in premature rupture of membranes at term: A randomized comparative trial. Journal of Obstetrics \& Gynaecology Research, 37(11), 1564-1571. doi:10.1111/j.1447-0756.2011.01575.x

Clausson, B., Gardosi, J., Francis, A., \& Cnattingius, S. (2001). Perinatal outcome in SGA births defined by customised versus population-based birthweight standards. BJOG: An International Journal of Obstetrics \& Gynaecology, 108(8), 830-834. doi:10.1111/j.1471-0528.2001.00205.x

Clinical Guidelines Panel. (2019). Induction of Labour in Aotearoa New Zealand: A clinical practice guideline. Retrieved from https://www.midwife.org.nz/wpcontent/uploads/2020/02/induction-of-labour-in-Aotearoa-New-Zealand-guideline2019.pdf

Dare, M. R., Middleton, P., Crowther, C. A., Flenady, V. J., \& Varatharaju, B. (2006). Planned early birth versus expectant management (waiting) for prelabour rupture of membranes at term (37 weeks or more). Cochrane database of systematic reviews (Online)(1). Retrieved from https://www.scopus.com/inward/record.uri?eid=2-s2.033645518624\&partnerID=40\&md5=1fc05a2e813d5dd568866ffffaa958d9

Darlow, B., Campbell, N., Austin, N., Chin, A., Grigg, C., Skidmore, C., ... Werno, A. (2015). The prevention of early-onset neonatal Group B Streptococcus infection: The New Zealand Consensus Guidelines 2014. New Zealand Medical Journal Retrieved from https://www.nzma.org.nz/journal/read-the-journal/all-issues/2010-2019/2015/vol128-no-1425-20-november-2015/6727

Dixon, L., \& Foureur, M. (2010). The vaginal examination during labour: is it of benefit or harm? New Zealand College of Midwives Journal, 42, 21+. Retrieved from https://link.gale.com/apps/doc/A251277462/AONE?u=learn\&sid=AONE\&xid=c886e1 $\underline{\mathrm{a} 0}$

Dixon, L., Prileszky, G., Guilliland, K., Miller, S., \& Anderson, J. (2014). Place of birth and outcomes for a cohort of low risk women in New Zealand: a comparison with 
Birthplace England. New Zealand College of Midwives Journal, 11+. Retrieved from https://link.gale.com/apps/doc/A499277578/AONE?u=learn\&sid=AONE\&xid=fOd990 $\underline{a b}$

Eickhoff, T. C., Klein, J. O., Daly, A. K., Ingall, D., \& Finland, M. (1964). Neonatal sepsis and other infections due to group B betahemolytic streptococci. New England Journal of Medicine(271), 1221-1228.

Ekart, J. (2014). Success to clinical audit. Whangarei, New Zealand: Northland District Health Board.

El-Helali, N., Giovangrandi, Y., Guyot, K., Chevet, K., Gutmann, L., \& Durand-Zaleski, I. (2012). Cost and effectiveness of intrapartum group $B$ streptococcus polymerase chain reaction screening for term deliveries. Obstetrics \& Gynecology, 119(4), 822-829. doi:10.1097/AOG.0b013e31824b1461

El-Helali, N., Habibi, F., Azria, E., Giovangrandi, Y., Autret, F., Durand-Zaleski, I., \& Le Monnier, A. (2019). Point-of-care intrapartum group B streptococcus molecular screening: effectiveness and costs. Obstetrics \& Gynecology, 133(2), 276-281. doi:10.1097/AOG.0000000000003057

El-Messidi, A., \& Cameron, A. (2010). Diagnosis of premature rupture of membranes: Inspiration from the past and insights for the future. Journal of Obstetrics and Gynaecology Canada, 32(6), 561-569. doi:10.1016/S1701-2163(16)34525-X

Fullston, E. F., Doyle, M. J., Higgins, M. F., \& Knowles, S. J. (2019). Clinical impact of rapid polymerase chain reaction (PCR) test for group B Streptococcus (GBS) in term women with ruptured membranes. Irish Journal of Medical Science (1971 -), 188(4), 12691274. doi:10.1007/s11845-019-01977-x

Garrard, J. (2011). Health Sciences literature review made easy (3rd ed.). Sudbury, MA: Jones \& Bartlett Learning, LLC.

Geethanath, R. M., Ahmed, I., Abu-Harb, M., Onwuneme, C., McGarry, K., \& Hinshaw, K. (2019). Intrapartum antibiotics for prolonged rupture of membranes at term to prevent Group B Streptococcal sepsis. Journal of Obstetrics and Gynaecology, 39(5), 619-622. doi:10.1080/01443615.2018.1550474

Gestation Network. (n.d.). Growth Charts. Retrieved from https://www.gestation.net/growthcharts.htm

Gomez-Lopez, N., Hernandez-Santiago, S., Lobb, A. P., Olson, D. M., \& Vadillo-Ortega, F. (2013). Normal and premature rupture of fetal membranes at term delivery differ in regional chemotactic activity and related chemokine/cytokine production. Reproductive Sciences, 20(3), 276-284. doi:10.1177/1933719112452473

Gonzalez-Perez, G., Hicks, A. L., Tekieli, T. M., Radens, C. M., Williams, B. L., \& LamouséSmith, E. S. N. (2016). Maternal Antibiotic Treatment Impacts Development of the Neonatal Intestinal Microbiome and Antiviral Immunity. The Journal of Immunology, 196(9), 3768. Retrieved from https://www.jimmunol.org/content/jimmunol/196/9/3768.full.pdf

Grigg, C. (2008). Working with women in pregnancy. In S. Pairman, J. Pincombe, C. Thorogood, \& S. Tracy (Eds.), Midwifery: Preparation for Practice (pp. 341-373). Marrickville, Australia: Elsevier.

Grigg, C., Tracy, S. K., Daellenbach, R., Kensington, M., \& Schmied, V. (2014). An exploration of influences on women's birthplace decision-making in new zealand: A mixed methods prospective cohort within the evaluating maternity units study. . BMC Pregnancy and Childbirth, 14(210). doi:10.1186/1471-2393-14-210 
Grimwood, K., Stone, P. R., Gosling, I. A., Green, R., Darlow, B. A., Lennon, D. R., \& Martin, D. R. (2002). Late antenatal carriage of group B Streptococcus by New Zealand women. Australian and New Zealand Journal of Obstetrics and Gynaecology, 42(2), 182-186. doi:10.1111/j.0004-8666.2002.00182.x

Güngördük, K., Asicioglu, O., Besimoglu, B., Güngördük, O. C., Yildirm, G., Ark, C., \& Sahbaz, A. (2012). Labor induction in term premature rupture of membranes: comparison between oxytocin and dinoprostone followed 6 hours later by oxytocin. American Journal of Obstetrics and Gynecology, 206(1), 60.e61 - 68. doi:10.1016/j.ajog.2011.07.035

Hannah, M. E., Ohlsson, A., Farine, D., Hewson, S. A., Hodnett, E. D., Myhr, T. L., . . Willan, A. R. (1996). Induction of labor compared with expectant management for prelabor rupture of the membranes at term. New England Journal of Medicine, 334(16), 10051010. doi:10.1056/NEJM199604183341601

Health and Disability Commissioner. (1996). Code of health and disability services consumers' rights. Retrieved from https://www.hdc.org.nz/your-rights/about-the-code/code-ofhealth-and-disability-services-consumers-rights/

Health and Disability System Review. (2020). Health and Disability System Review - Final Report - Pūrongo Whakamutunga. . Wellington, New Zealand: HDSR Retrieved from https://systemreview.health.govt.nz/assets/Uploads/hdsr/health-disability-systemreview-final-report.pdf

Health Navigator New Zealand. (2020). C-reactive protein test. Retrieved from https://www.healthnavigator.org.nz/health-a-z/c/c-reactive-protein-test/

Healthpoint Ltd. (n.d.). Northland DHB Maternity Services. Retrieved from https://www.healthpoint.co.nz/public/maternity/northland-dhb-maternity-services/

Helmig, R. B., \& Gertsen, J. B. (2019). Intrapartum PCR-assay for detection of Group B Streptococci (GBS). European journal of obstetrics \& gynecology and reproductive biology: $X, 4,100081$. doi:10.1016/j.eurox.2019.100081

Hiersch, L., Krispin, E., Aviram, A., Mor-Shacham, M., Gabbay-Benziv, R., Yogev, Y., \& Ashwal, E. (2017). Predictors for prolonged interval from premature rupture of membranes to spontaneous onset of labor at term. Journal of Maternal-Fetal \& Neonatal Medicine, 30(12), 1465-1470. doi:10.1080/14767058.2016.1219992

Hokianga Health Enterprise Trust. (2018). Annual report for the year ending 30 June 2018. Kaikohe, New Zealand: Hokianga Health Enterprise Trust

Hudson, M., Milne, M., Reynolds, P., Russell, K., \& Smith, B. (n.d.). Te Ara Tika: Guidelines for Maori research ethics: A framework for researchers and ethics committee members. Health Research Council of New Zealand. Retrieved from http://www.hrc.govt.nz/sites/default/files/Te\%20Ara\%20Tika\%20Guidelines\%20for \%20Maori\%20Research\%20Ethics.pdf

Ismail, A. Q. T., \& Lahiri, S. (2013). Management of prelabour rupture of membranes (PROM) at term. Journal of Perinatal Medicine, 41(6), 647-649. doi:10.1515/jpm-2013-0078

Kaiser Permanente. (2020). Neonatal Early-Onset Sepsis Calculator. Retrieved from https://neonatalsepsiscalculator.kaiserpermanente.org/InfectionProbabilityCalculat or.aspx

Khalil, M. R., Thorsen, P. B., Møller, J. K., \& Uldbjerg, N. (2018). Number of colony forming units in urine at 35-37 weeks' gestation as predictor of the vaginal load of Group B Streptococci at birth. European Journal of Obstetrics \& Gynecology and Reproductive Biology, 223, 68-71. doi:https://doi.org/10.1016/j.ejogrb.2018.02.013 
Klingenberg, C., Kornelisse, R. F., Buonocore, G., Maier, R. F., \& Stocker, M. (2018). CultureNegative Early-Onset Neonatal Sepsis - At the Crossroad Between Efficient Sepsis Care and Antimicrobial Stewardship. Frontiers in pediatrics, 6, 285-285. doi:10.3389/fped.2018.00285

Kronast, H., \& Lewis, L. (2020, 29 July 2020). Northland floods: Midwives pack chainsaws, ride dirtbikes to get to mothers in need. Newshub. Retrieved from https://www.newshub.co.nz/home/new-zealand/2020/07/northland-floodsmidwives-pack-chainsaws-ride-dirtbikes-to-get-to-mothers-in-need.html

Kulhan, N., \& Kulhan, M. (2019). Labor induction in term nulliparous women with premature rupture of membranes: oxytocin versus dinoprostone. Archives of Medical Science, 15(4), 896-901. doi:10.5114/aoms.2018.76115

Kunt, C., Kanat-Pektas, M., Gungor, A. N., Kurt, R. K., Ozat, M., Gulerman, C., . . Omar, S. Z. (2010). Randomized trial of vaginal prostaglandin E2 versus oxytocin for labor induction in term premature rupture of membranes. Taiwanese Journal of Obstetrics \& Gynecology., 49(1), 57 - 61. doi:10.1016/S1028-4559(10)60010-1

Kurasawa, K., Yamamoto, M., Usami, Y., Mochimaru, A., Mochizuki, A., Aoki, S., . . Hirahara, F. (2014). Significance of cervical ripening in pre-induction treatment for premature rupture of membranes at term. Journal of Obstetrics \& Gynaecology Research, 40(1), 32-39. doi:10.1111/jog.12116

LabPlus. (2018). Antimicrobial Susceptibility Test Results for Gram-positive isolates recovered from adult and paediatric clinical specimens: 2018. Retrieved from http://www.labplus.co.nz/assets/Uploads/2018-Gram-Positive-AntimicrobialReport.pdf

Labtests. (2018). Which swab/specimen for which test? Retrieved from https://www.labtests.co.nz/wp-content/uploads/sites/2/2019/11/4.4-Which-Swabspecimen-for-Which-Test.pdf

Larrañaga-Azcárate, C., Campo-Molina, G., Pérez-Rodríguez, A. F., \& Ezcurdia-Gurpegui, M. (2008). Dinoprostone vaginal slow-release system (Propess ${ }^{\circledR}$ ) compared to expectant management in the active treatment of premature rupture of the membranes at term: Impact on maternal and fetal outcomes. Acta Obstetricia et Gynecologica Scandinavica, 87(2), 195-200. doi:10.1080/00016340701837421

Lee, K. A., Lee, S. M., Yang, H. J., Park, C. W., Mazaki-Tovi, S., Yoon, B. H., \& Romero, R. (2011). The frequency of meconium-stained amniotic fluid increases as a function of the duration of labour. J Matern Fetal Neonatal Med, 24, 880-885. doi:10.3109/14767058.2010.531329

Li, K., Wang, Y., Li, H., \& Yang, H. (2011). A study of 579 pregnant women with premature rupture of membranes at term. International Journal of Gynecology and Obstetrics, 112(1), 45-47. doi:10.1016/j.ijgo.2010.07.026

Liberati, A., Altman, D. G., Tetzlaff, J., Mulrow, C., Gøtzsche, P. C., Ioannidis, J. P. A., . . Moher, D. (2009). The PRISMA Statement for Reporting Systematic Reviews and MetaAnalyses of Studies That Evaluate Health Care Interventions: Explanation and Elaboration. PLOS Medicine, 6(7), e1000100. doi:10.1371/journal.pmed.1000100

Mahomed, K., Wild, K., \& Weekes, C. R. (2018). Prostaglandin gel versus oxytocin - prelabour rupture of membranes at term - A randomised controlled trial. Aust $\mathrm{N} Z \mathrm{~J}$ Obstet Gynaecol. doi:10.1111/ajo.12788 
Malik, H. Z., Khawaja, N. P., Zahid, B., \& Rehman, R. (2010). Sublingual versus oral misoprostol for induction of labour in prelabour rupture of membranes at term. $J$ Coll Physicians Surg Pak, 20(4), 242-245. doi:04.2010/JCPSP.242245

Marowitz, A., \& Jordan, R. (2007). Midwifery Management of Prelabor Rupture of Membranes at Term. 52(3), 199-206. doi:10.1016/j.jmwh.2006.12.012

McCowan, L., Cowan, J., \& Stein de-Laat, M. (2019). Recognition and management of small for gestational age pregnancies. The University of Auckland. Auckland, New Zealand. Retrieved from https://objectext.auckland.ac.nz/figshare/18723794/RecognitionManagementofSGA October312019final .pdf

Melin, P. (2011). Neonatal group B streptococcal disease: From pathogenesis to preventive strategies. Clinical Microbiology and Infection, 17(9), 1294-1303. doi:10.1111/j.14690691.2011.03576.x

Mertens, D. M., \& Wilson, A. T. (2012). Program Evaluation Theory and Practice : A Comprehensive Guide. New York, UNITED STATES: Guilford Publications.

Middleton, P., Shepherd, E., Flenady, V., McBain, R. D., \& Crowther, C. A. (2017). Planned early birth versus expectant management (waiting) for prelabour rupture of membranes at term (37 weeks or more). Cochrane Database of Systematic Reviews(1). doi:10.1002/14651858.CD005302.pub3

Midwifery and Maternity Provider Organisation. (2016). Report on New Zealand's MMPO midwives care activities and outcomes. Christchurch, New Zealand: New Zealand College of Midwives. Retrieved from https://www.midwife.org.nz/wpcontent/uploads/2019/01/MMPO-report-2016.pdf

Midwifery Council of New Zealand. (2019). Midwifery workforce survey 2019. Wellington, New Zealand: Midwifery Council Retrieved from https://www.midwiferycouncil.health.nz/sites/default/files/sitedownloads/Workforce\%20Survey\%202019\%20\%20-\%20Complete.pdf

Midwifery Council of New Zealand. (2020). Aotearoa Midwifery Project. Retrieved from https://www.midwiferycouncil.health.nz/aotearoa-midwifery-project

Midwifery Council of New Zealand. (n.d.). Midwifery Council: Guardians of professional standards. Retrieved from https://www.midwiferycouncil.health.nz/

Midwifery Employment Representation and Advisory Service. (2014). Midwifery Staffing Standards. Retrieved from https://meras.midwife.org.nz/wpcontent/uploads/sites/4/2019/06/MERAS-Mid-Staff-Stds-2014.pdf

Midwifery Employment Representation and Advisory Service. (2019). Māori midwives in the news. Retrieved from https://meras.midwife.org.nz/news/maori-midwives-in-thenews/

Ministry of Health. (1999). Breastfeeding definitions for monitoring the national health outcome targets in New Zealand. Wellington, New Zealand: Ministry of Health

Ministry of Health. (2007). Maternity Services: Notice pursuant to Section 88 of the New Zealand Public Health and Disability Act 2000. Wellington, New Zealand: Department of Internal Affairs. Retrieved from https://www.health.govt.nz/publication/primarymaternity-services-notice-2007

Ministry of Health. (2012). Guidelines for consultation with obstetric and related medical services (Referral Guidelines). Wellington, New Zealand: Ministry of Health

Ministry of Health. (2018a). District Health Boards. Retrieved from https://www.health.govt.nz/new-zealand-health-system/key-health-sector- 
organisations-and-people/district-healthboards?mega=NZ\%20health\%20system\&title=District\%20health\%20boards

Ministry of Health. (2018b). Hokianga Health Enterprise Trust. Retrieved from https://www.health.govt.nz/your-health/services-and-support/health-careservices/maori-health-provider-directory/north-island-maori-healthproviders/northland-maori-health-providers/hokianga-health-enterprise-trust

Ministry of Health. (2019a). Maternity Services. Retrieved from https://www.health.govt.nz/our-work/life-stages/maternity-services

Ministry of Health. (2019b). New Zealand Maternity Clinical Indicators 2017. Ministry of Health Retrieved from https://www.health.govt.nz/system/files/documents/publications/new-zealandmaternity-clinical-indicators-2017-jul19.pdf

Ministry of Health. (2019c). Wai 2575 Māori health trends report Wellington, New Zealand: Ministry of Health Retrieved from https://www.health.govt.nz/system/files/documents/publications/wai-2575-maorihealth-trends-report-04mar2020.pdf

Ministry of Health. (2020a). Pregnancy. Retrieved from https://www.health.govt.nz/yourhealth/pregnancy-and-kids/pregnancy

Ministry of Health. (2020b). Whakamaua: Māori Health Action Plan 2020-2025. Wellington, New Zealand: Ministry of Health Retrieved from https://www.health.govt.nz/system/files/documents/publications/whakamauamaori-health-action-plan-2020-2025-2.pdf

Ministry of Justice. (1993). Privacy Act 1993. Wellington, New Zealand: Ministry of Justice, Retrieved from http://www.legislation.govt.nz/act/public/1993/0028/latest/DLM297038.html

Money, D., \& Allen, V. M. (2016). The Prevention of Early-Onset Neonatal Group B Streptococcal Disease. Journal of Obstetrics and Gynaecology Canada., 38(12 Supplement), S326 - S335. doi:10.1016/j.jogc.2016.09.042

Moorfield, J. C. (n.d.). Te Aka Online Māori Dictionary. Retrieved from https://maoridictionary.co.nz/

Morse, J. M., \& Field, P. (1995). Qualitative research methods for health professionals. Thousand Oaks, CA: Sage Publications.

Mozurkewich, E., Horrocks, J., Daley, S., Von Oeyen, P., Halvorson, M., Johnson, M., . . et al. (2003). The MisoPROM study: a multicenter randomized comparison of oral misoprostol and oxytocin for premature rupture of membranes at term. American Journal of Obstetrics and Gynecology., 189(4), 1026 - 1030. Retrieved from https://www.ajog.org/article/S0002-9378(03)00845-7/fulltext

Mueller, N. T., Bakacs, E., Combellick, J., Grigoryan, Z., \& Dominguez-Bello, M. G. (2015). The infant microbiome development: mom matters. Trends Mol Med, 21(2), 109-117. doi:10.1016/j.molmed.2014.12.002

Mynarek, M., Bjellmo, S., Lydersen, S., Strand, K. M., Afset, J. E., Andersen, G. L., \& Vik, T. (2020). Prelabor rupture of membranes and the association with cerebral palsy in term born children: a national registry-based cohort study. BMC Pregnancy and Childbirth, 20(1), 67. doi:10.1186/s12884-020-2751-3 
Nabhan, A. F., Elhelaly, A., \& Elkadi, M. (2014). Antibiotic prophylaxis in prelabor spontaneous rupture of fetal membranes at or beyond 36 weeks of pregnancy. 124(1), 59-62. doi:10.1016/j.ijgo.2013.07.018

Nagpal, M. B., Raghunandan, C., \& Saili, A. (2009). Oral misoprostol versus intracervical prostaglandin E2 gel for active management of premature rupture of membranes at term. International Journal of Gynaecology and Obstetrics., 106(1), 23 - 26. doi:10.1016/j.ijgo.2009.03.014

Namli Kalem, M., Köşüş, A., Kamalak, Z., Köşüş, N., \& Kalem, Z. (2017). Factors affecting the rates of caesarean sections in cases with premature rupture of membranes (PROM) at term. Journal of Obstetrics \& Gynaecology, 37(5), 585-590. doi:10.1080/01443615.2016.1274291

NDHB. (2017). 2017 Maternity Quality and Safety Annual Report. Whangarei: Northland District Health Board

New Zealand College of Midwives. (2015). Midwives Handbook for Practice. Christchurch, New Zealand: New Zealand College of Midwives

New Zealand College of Midwives. (2016). Consensus Statement: Laboratory testing/screening. Christchurch, New Zealand: New Zealand College of Midwives Retrieved from https://www.midwife.org.nz/wpcontent/uploads/2019/05/Laboratory-Testing-Screening.pdf

New Zealand College of Midwives. (2020). Find your midwife. Retrieved from https://www.findyourmidwife.co.nz/midwives/northland?month=all\&name=\&birthP lace=all\&area=all\&lang=all

Northland District Health Board. (2017). 2017 Maternity Quality and Safety Annual Report. Whangarei, New Zealand: Northland District Health Board Retrieved from https://www.northlanddhb.org.nz/assets/Communications/Publications/NorthlandDHB-MQSP-Annual-Report-2016-2017.pdf

Northland District Health Board. (2018a). 2018 Maternity Quality and Safety Annual Report. Whangarei, New Zealand: Northland District Health Board Retrieved from https://www.northlanddhb.org.nz/assets/Communications/Publications/MaternityQuality-and-Safety-Annual-Report-2018.pdf

Northland District Health Board. (2018b). Our vision, mission and values. Retrieved from https://www.northlanddhb.org.nz/about-us/vision-mission-objectives-andstrategies/our-vision-mission-and-values/

Northland District Health Board. (2019). Northland District Health Board Annual Report 2019. Whangarei, New Zealand: Northland District Health Board Retrieved from https://www.northlanddhb.org.nz/assets/Communications/Publications/NorthlandDHB-Annual-Report-2019.pdf

Northland District Health Board. (2020). Maternity Service. Retrieved from https://www.northlanddhb.org.nz/our-services/our-hospitals/whangarei-hospitalservices/maternity-service/

Northland Pathology. (2020). Collection Centres. Retrieved from https://norpath.co.nz/collection-centres/

Nottingham Clinical Trials Unit. (2020). Routine testing for Group B streptococcus in pregnancy (GBS3 trial). Retrieved from http://www.isrctn.com/ISRCTN49639731

Ohlsson, A., \& Shah, V. S. (2014). Intrapartum antibiotics for known maternal Group B streptococcal colonization. Cochrane Database of Systematic Reviews(6). doi:10.1002/14651858.CD007467.pub4 
On Track Network. (2020). Newsletter of the On Track Network: August 2020 [Press release] Palasanthiran, P., Starr, M., Jones, C., \& Giles, M. E. (2014). Management of perinatal infections. Retrieved from https://www.asid.net.au/documents/item/368

Parliamentary Counsel Office. (2000). New Zealand Public Health and Disability Act 2000. Wellington: Parliamentary Counsel Office Retrieved from http://www.legislation.govt.nz/act/public/2000/0091/latest/DLM80051.html

Health Practitioners Competence Assurance Act 2003, (2003).

Passos, F., Cardoso, K., Coelho, A. M., Graça, A., Clode, N., \& Mendes da Graça, L. (2012). Antibiotic prophylaxis in premature rupture of membranes at term: a randomized controlled trial. Obstetrics and Gynecology, 120(5), 1045 - 1051. doi:10.1097/AOG.0b013e31826e46bc

Patterson, J. A., Keuler, N. S., \& Olson, B. H. (2019). The effect of maternity practices on exclusive breastfeeding rates in U.S. hospitals. Maternal \& child nutrition, 15(1), e12670-e12670. doi:10.1111/mcn.12670

Perinatal and Maternal Mortality Review Committee. (2018). Twelfth annual report of the perinatal and maternal mortality review committee: Reporting mortality 2016. . Wellington, New Zealand: Health Quality and Safety Commission Retrieved from https://www.hqsc.govt.nz/assets/PMMRC/Publications/12th-PMMRC-reportfinal.pdf

Poornima, B., \& Dharma Reddy, D. B. (2011). Premature rupture of membranes at term: immediate induction with PGE 2 gel compared with delayed induction with oxytocin. Journal of obstetrics and gynaecology of india, 61(5), 516 - 518. doi:10.1007/s13224011-0086-8

Posavac, E. J., \& Carey, R. G. (2007). Program evaluation: Methods and case studies. Upper Saddle River, New Jersey: Pearson Education.

Pourali, L., Saghafi, N., Eslami Hasan Abadi, S., Tara, F., Vatanchi, A. M., \& Motamedi, E. (2018). Induction of labour in term premature rupture of membranes; oxytocin versus sublingual misoprostol; a randomised clinical trial. Journal of Obstetrics \& Gynaecology, 38(2), 167-171. doi:10.1080/01443615.2017.1329284

Qiagen Sciences. (2019). Amnisure: The technology. Retrieved from http://www.amnisure.com/tech/

Radoff, K. A. (2014). Orally Administered Misoprostol for Induction of Labor with Prelabor Rupture of Membranes at Term. Journal of Midwifery \& Women's Health, 59(3), 254263. doi:10.1111/jmwh.12195

Ramesh Babu, S., Mcdermott, R., Farooq, I., Le Blanc, D., Ferguson, W., Mccallion, N., . . . Eogan, M. (2018). Screening for group B Streptococcus (GBS) at labour onset using PCR: accuracy and potential impact - a pilot study. Journal of obstetrics and gynaecology, 38(1), 49-54. doi:10.1080/01443615.2017.1328490

Rawat, R., Divedi, P., Debbarma, S., Vishwakarma, S., \& Mittal, N. (2018). A comparative study between active and expectant management of premature rupture of membranes at term on fetomaternal and perinatal outcome in rural population. International Journal of Reproduction, Contraception, Obstetrics and Gynecology, 7(6), 2393. doi:10.18203/2320-1770.ijrcog20182356

Reeves, S., \& Bernstein, I. (2008). Effects of maternal tobacco-smoke exposure on fetal growth and neonatal size. Expert Review of Obstetrics \& Gynecology, 3(6), 719-730. doi:10.1586/17474108.3.6.719 
Saccone, G., \& Berghella, V. (2015). Antibiotic prophylaxis for term or near-term premature rupture of membranes: Metaanalysis of randomized trials. American Journal of Obstetrics and Gynecology, 212(5), 627.e621-627.e629. doi:10.1016/j.ajog.2014.12.034

Sadeh-Mestechkin, D., Samara, N., Wiser, A., Markovitch, O., Shechter-Maor, G., \& BironShental, T. (2016). Premature rupture of the membranes at term: time to reevaluate the management. Archives of Gynecology and Obstetrics, 294(6), 1203-1207. doi:10.1007/s00404-016-4165-z

Schafer, R., \& Phillippi, J. C. (2020). Group B Streptococcal Bacteriuria in Pregnancy: An Evidence-Based, Patient-Centered Approach to Care. Journal of Midwifery \& Women's Health, 65(3), 376-381. doi:10.1111/jmwh.13085

Seaward, P. G. R., Hannah, M. E., Myhr, T. L., Farine, D., Ohlsson, A., Wang, E. E., . . Ohel, G. (1998). International Multicenter Term PROM Study: Evaluation of predictors of neonatal infection in infants born to patients with premature rupture of membranes at term. American Journal of Obstetrics and Gynecology., 179(3), 635-639. doi:https://doi.org/10.1016/S0002-9378(98)70056-0

Seedat, F., Geppert, J., Stinton, C., Patterson, J., Freeman, K., Johnson, S. A., . . Taylor-Phillips, S. (2019). Universal antenatal screening for group B streptococcus may cause more harm than good. BMJ 2019, 364(1463). doi:https://doi.org/10.1136/bmj.1463

Selmer-Olsen, T., Lydersen, S., \& Mørkved, S. (2007). Does acupuncture used in nulliparous women reduce time from prelabour rupture of membranes at term to active phase of labour? A randomised controlled trial. Acta Obstetricia et Gynecologica Scandinavica, 86(12), 1447-1452. doi:10.1080/00016340701645287

Shah, K., \& Doshi, H. (2012). Premature Rupture of Membrane at Term: Early Induction Versus Expectant Management. The Journal of Obstetrics and Gynecology of India., 62(2), 172-175. doi:10.1007/s13224-012-0172-6

Simkin, P. (2010). The Fetal Occiput Posterior Position: State of the Science and a New Perspective. Birth, 37(1), 61-71. doi:10.1111/j.1523-536X.2009.00380.x

Simkin, P., \& Ancheta, R. (2011). Prolonged active phase of labor. In P. Simkin \& R. Ancheta (Eds.), The labor progress handbook. (pp. 124-172). Chichester, England: WileyBlackwell.

Singh, T., Barnes, E. H., \& Isaacs, D. (2019). Early-onset neonatal infections in Australia and New Zealand, 2002-2012. Archives of Disease in Childhood, Fetal and Neonatal Edition, 104(3), 248-252. doi:10.1136/archdischild-2017-31467

Sinha, S., Talaulikar, V. S., \& Arulkumaran, S. (2018). Malpositions and malpresentations of the fetal head. Obstetrics, Gynaecology \& Reproductive Medicine, 28(3), 83-91. doi:https://doi.org/10.1016/i.ogrm.2018.01.001

Smaill, F. M., \& Vazquez, J. C. (2019). Antibiotics for asymptomatic bacteriuria in pregnancy. Cochrane Database of Systematic Reviews(11). doi:10.1002/14651858.CD000490.pub4

Smith, L. J. (2007). Impact of birthing practices on the breastfeeding dyad. J Midwifery Womens Health, 2007(6), 621-630. doi:10.1016/j.jmwh.2007.07.019

Social Policy Evaluation and Research Unit. (2015). Evaluation standards for Aotearoa New Zealand. Wellington, New Zealand: Aotearoa New Zealand Evaluation Association. Retrieved from http://anzea.org.nz//wp-content/uploads/2014/08/ANZEA-SuperuEvaluation-standards-final-020415.pdf 
Song, J., Lu, J., Wang, E., Lu, M., An, Z., Liu, Y., . . Wu, W. (2019). Short-term effects of ambient temperature on the risk of premature rupture of membranes in Xinxiang, China: A time-series analysis. Science of the Total Environment, 689, 1329-1335. doi:10.1016/j.scitotenv.2019.06.457

Stats New Zealand. (2020). 2018 Census place summaries: Northland Region. Retrieved from https://www.stats.govt.nz/tools/2018-census-place-summaries/northland-region

Stevenson, K., Filoche, S., Cram, F., \& Lawton, B. (2020). Te ha o whanau: A culturally responsive framework of maternity care.

http://ezproxy.auckland.ac.nz/login?url=https://search-proquestcom.ezproxy.auckland.ac.nz/docview/2419751539?accountid=8424 The New Zealand Medical Journal (Online), 133(1517), 66-72. Retrieved from https://www.nzma.org.nz/journal-articles/te-ha-o-whanau-a-culturally-responsiveframework-of-maternity-care

Strunk, T., Buchiboyina, A., Sharp, M., Nathan, E., Doherty, D., \& Patole, S. (2018). Implementation of the Neonatal Sepsis Calculator in an Australian Tertiary Perinatal Centre. Neonatology, 113(4), 379-382. doi:10.1159/000487298

Tanaka, S., Kobayashi, T., Songjinda, P., Tateyama, A., Tsubouchi, M., Kiyohara, C., . . . Nakayama, J. (2009). Influence of antibiotic exposure in the early postnatal period on the development of intestinal microbiota. FEMS Immunology \& Medical Microbiology, 56(1), 80-87. doi:10.1111/j.1574-695X.2009.00553.x

Tara, F., Rayman, M. P., Boskabadi, H., Ghayour-Mobarhan, M., Sahebkar, A., Yazarlu, O., . . . et al. (2010). Selenium supplementation and premature (pre-labour) rupture of membranes: a randomised double-blind placebo-controlled trial. Journal of Obstetrics and Gynaecology, 30(1), 30 - 34. doi:10.3109/01443610903267507

The National Institute for Health and Care Excellence. (2014). Intrapartum care for healthy women and babies. National Institute for Health and Care Excellence Retrieved from https://www.nice.org.uk/guidance/cg190

The Royal Australian and New Zealand College of Obstetricians and Gynaecologists. (2016). Maternal Group B Streptococcus in pregnancy: screening and management. Retrieved from https://www.ranzcog.edu.au/RANZCOG SITE/media/RANZCOGMEDIA/Women\%27s\%20Health/Statement\%20and\%20guidelines/ClinicalObstetrics/Maternal-Group-B-Streptococcus-in-pregnancy-screening-andmanagement-(C-Obs-19)-Review-March-2016.pdf?ext=.pdf

The Royal Australian and New Zealand College of Obstetricians and Gynaecologists. (2017). Term prelabour rupture of membranes (Term PROM). Retrieved from https://www.ranzcog.edu.au/RANZCOG_SITE/media/RANZCOG-

MEDIA/Women\%27s\%20Health/Statement\%20and\%20guidelines/ClinicalObstetrics/Term-Prelabour-Rupture-of-Membranes-(Term-Prom)-(C-Obs-36)-review2017.pdf?ext=.pdf

Thorogood, C., \& Donaldson, C. (2008). Disturbances in the rhythm of labour. In S. Pairman, J. Pincombe, C. Thorogood, \& S. Tracy (Eds.), Midwifery: Preparation for Practice (pp. 679-716). Marrickville, Australia: Elsevier.

Towers, C. V., Yates, A., Zite, N., Smith, C., Chernicky, L., \& Howard, B. (2017). Incidence of fever in labor and risk of neonatal sepsis. American Journal of Obstetrics and Gynecology, 216(6), 596.e591-596.e595. doi:https://doi.org/10.1016/i.ajog.2017.02.022 
Tran, S. H., Cheng, Y. W., Kaimal, A. J., \& Caughey, A. B. (2008). Length of rupture of membranes in the setting of premature rupture of membranes at term and infectious maternal morbidity. American Journal of Obstetrics and Gynecology, 198(6), 700.e701-700.e705. doi:10.1016/j.ajog.2008.03.031

Waitangi Tribunal. (2019). Hauora: Report on Stage One of the Health Services and Outcomes Kaupapa Inquiry. Lower Hutt, New Zealand: Legislation Direct Retrieved from https://forms.justice.govt.nz/search/Documents/WT/wt DOC 152801817/Hauora\% 20W.pdf

White, P., Gunston, J., Salmond, C., Atkinson, J., \& Crampton, P. (2008). Atlas of socioeconomic deprivation in New Zealand NZDep2006. Wellington: Ministry of Health Retrieved from

https://www.health.govt.nz/system/files/documents/publications/nzdep2006 text. pdf

Wise, M., Campbell, N., \& Darlow, B. (2015). Prevention of neonatal early onset GBS sepsis: A clear protocol is better than none - or several. The New Zealand Medical Journal, 128(1425). Retrieved from https://www.nzma.org.nz/journal/read-the-journal/allissues/2010-2019/2015/vol-128-no-1425-20-november-2015/6720

World Health Organisation. (1996). Care in normal birth: A practical guide. Geneva, Switzerland: World Health Organisation.

Xia, H., Li, X., Li, X., Liang, H., \& Xu, H. (2015). The clinical management and outcome of term premature rupture of membrane in East China: Results from a retrospective multicenter study. International Journal of Clinical and Experimental Medicine, 8(4), 6212-6217. Retrieved from https://www.ncbi.nlm.nih.gov/pmc/articles/PMC4483966/pdf/ijcem0008-6212.pdf

Yasmin, S., Yasmin, A., Khattak, N. N., Karim, R., \& Raees, M. (2013). Active versus conservative management of prelabour rupture of membranes at term. Journal of Postgraduate Medical Institute., 27(1), 63 - 68. Retrieved from https://www.cochranelibrary.com/central/doi/10.1002/central/CN-00906950/full

Zea-Vera, A., \& Ochoa, T. J. (2015). Challenges in the diagnosis and management of neonatal sepsis. Journal of tropical pediatrics, 61(1), 1-13. doi:10.1093/tropej/fmu079 


\title{
Appendix 1: Letter of introduction and confirmation of permission to identify NDHB in thesis
}

\author{
Deb Pittam \\ Midwifery Director and Service Manager - Maternity Service \\ Private Bag 9742 \\ Whangarei NZ
}

4 April 2019

To the Midwifery Director and Service Manager - Maternity Service, NDHB

This letter is to introduce Rebecca Hay, who is completing a Master of Health Research (Midwifery) through Victoria University of Wellington. Her supervisors are Dr Robyn Maude and Dr Sue Calvert. Her topic is "Management of prelabour rupture of membranes at term (PROM) at Northland District Health Board". Rebecca has approval from the VUW Ethics Committee and has locality approval from NDHB for this study. She has also consulted with Māori at VUW and NDHB, including a kōrero with NZCOM Kuia and local LMC, Crete Cherrington.

The aims of this research are:

1. Analyse and compare outcomes for women and babies affected by PROM at NDHB with those of wider NZ;

2. Discuss the results found within the context of NDHB; and

3. Explore any benefits and drawbacks of the NDHB approach.

Rebecca is conducting a quantitative evaluation study and will perform a retrospective audit of clinical notes of all women experiencing PROM in NDHB within a one year period. It is estimated that this will be 100-130 women, including births at Te Kotuku and the NDHB primary centres. Rebecca will require your assistance to identify the records she needs to audit, and to negotiate appropriate workspaces while she visits NDHB facilities. Rebecca will be ready to start this process in the next few months and envisages visiting facilities for one day per week over 2-3 months, until data collection is completed. In addition, Rebecca plans to compare her outcome measures with those of unaffected women at NDHB and will seek anonymised Solutions Plus aggregate data. She will need your assistance with this. She will discuss her outcome measures and audit measures with you before commencing data collection.

Results will be shared with NDHB and could be utilised as part of the routine policy review process. As well as producing a thesis, Rebecca intends to respectfully publish from this work and to present her findings at midwifery conference(s). I support Rebecca's research, and approve the naming of Northland District Health Board as the location of this study.

Please continue to positively support Rebecca in her research.

Kind regards,

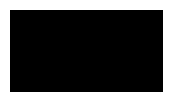

Deb Pittam

Midwifery Director and Service Manager - Maternity Service 


\section{Appendix 2: New Zealand DHB PROM and GBS Policy References}

Auckland District Health Board. (2015). Rupture of membranes in pregnancy. (NMP200/SSM/056). Auckland, New Zealand: Auckland District Health Board

Auckland District Health Board. (2017). Group B Streptococcus (GBS) - Prevention of early-onsent neonatal infection. (NMP200/SSM/054 - v05.00). Auckland, New Zealand: Auckland District Health Board

Bailey, D. (2015). Prelabour rupture of membranes at term (PROM). Whangarei, New Zealand: Northland District Health Board

Bailey, D. (2018). Group B Streptococcus - Prevention of early onset neonatal infection. Whangarei, New Zealand: Northland District Health Board.

Bay of Plenty District Health Board. (2017). Group B Streptococcus (GBS) Prevention of early onsent neonatal infection. (Mat.G1.1). Tauranga, New Zealand: Bay of Plenty District Health Board

Bay of Plenty District Health Board. (2017). Induction of Labour (IOL). (Mat.12.2). Tauranga, New Zealand: Bay of Plenty District Health Board

Bay of Plenty District Health Board. (2018). Rupture of membranes in pregnancy. (Mat.R1.1). Tauranga, New Zealand: Bay of Plenty District Health Board

Camano, I., Anderson, K., Dudley, N., \& Wright, P. (2017). Rupture of membranes in pregnancy. $(5772$ V01). Hamilton, New Zealand: Waikato District Health Board.

Camano, I., \& Wright, P. (2017). Prevention of neonatal Group B Streptococcus infection (GBS). (2262 v08). Hamilton, New Zealand: Waikato District Health Board

Canterbury District Health Board. (2017). Group B Streptococcus - management and prophylactic antibiotics in labour. (WCH/GLM0032 (231554)). Christchurch, New Zealand: Canterbury District Health Board

Canterbury District Health Board. (2017). Pre-labour rupture of membranes at term. (WCH/GLM0043 (231027) ). Christchurch, New Zealand: Canterbury District Health Board

Counties Manukau District Health Board. (2019). Group B Streptococcus - prevention of early onset neonatal infection. (A2783). Auckland, New Zealand: Counties Manukau District Health Board.

Counties Manukau District Health Board. (2019). Spontaneous rupture of membranes at term. (A2797). Auckland, New Zealand: Counties Manukau District Health Board

Dockrill, J. (2018). Group B Streptococcus infection - prevention and treatment. Timaru, New Zealand: South Canterbury District Health Board.

Fisher, D. (2014). Group B Streptococcus screening and management (Maternity). Nelson, New Zealand: Nelson Marlborough District Health Board

Forest, K. (2014). Group B Strep colonized women in labour. Masterton, New Zealand: Wairarapa District Health Board.

Hauora Tairāwhiti. (2015). The prevention of neonatal Group B Streptococcal Infection (GBS). Gisborne, New Zealand: Hauora Tairāwhiti

Hauora Tairāwhiti. (2017). Pre-labour rupture of the membranes at term. Gisborne, New Zealand: Hauora Tairāwhiti

Hawke's Bay District Health Board. (2015). Pre-labour spontaneous rupture of membranes at term (37-42 weeks) PSROM. (WCYS/MATPPM/8000). Hastings, New Zealand: Hawke's Bay District Health Board.

Hutt Maternity. (2013). Pre-labour spontaneous rupture of membranes at term. (MATY055). Lower Hutt, New Zealand: Hutt Valley District Health Board Retrieved from www.huttmaternity.org.nz/content/e87e0257-8a22-4852-9835-c002bf0d484d.cmr

Hutt Maternity. (2019). Group B Streptococcus (GBS) management and prophylactic antibiotics in labour. (MATY030). Lower Hutt, New Zealand: Hutt Valley District Health Board Retrieved from http://www.huttmaternity.org.nz/content/b69e4296-070a-4cbb-b504-8f8fc4c4bebd.cmr 
Lakes District Health Board. (2015). Pre-labour rupture of membranes guidelines (includes term and preterm rupture of membranes). (43549). Taupo, New Zealand: Lakes District Health Board

Maternity Clinical Guidelines Group. (2013). Induction of Labour. Nelson, New Zealand: Nelson Marlborough District Health Board

Mitchell, K. (2018). Induction of labour. Masterton, New Zealand.: Wairarapa District Health Board.

Read, L. (2015). Spontaneous rupture of membranes pre-labour. Masterton, New Zealand.: Wairarapa District Health Board.

Sangalli, M., \& Frei, C. (2015). Prevention of early-onset neonatal Group B Streptococcal disease. (1.530). Wellington, New Zealand: Capital and Coast District Health Board

Sangalli, M., \& Frei, C. (2015). Spontaneous rupture of membranes. (1.532). Wellington, New Zealand: Capital and Coast District Health Board

Southern District Health Board. (2015). Management of ruptured membranes before and after 37 weeks gestation (District). (21662). Dunedin, New Zealand: Southern District Health Board.

Taranaki District Health Board. (2018). Pre-labour rupture of membranes at term (PROM). (730-0571037). New Plymouth, New Zealand: Taranaki District Health Board.

Waitemata District Health Board. (2016). Induction of labour (IOL). (0125-12-001). Auckland, New Zealand: Waitemata District Health Board.

Waitemata District Health Board. (2018). Group B Streptococcus (GBS). (0125-07-001). Auckland, New Zealand: Waitemata District Health Board.

Werkmeister, G. (2016). Prolonged rupture of membranes (PROM) from 37+ weeks. Timaru, New Zealand: South Canterbury District Health Board

Whanganui District Health Board. (2016). Pre-labour rupture of membranes at term guideline. (WDHB2296 V4). Whanganui, New Zealand: Whanganui District Health Board.

Whanganui District Health Board. (2018). Induction of labour policy. (WDHB-1959 V1). Whanganui, New Zealand: Whanganui District Health Board.

Whanganui District Health Board. (2018). The prevention of early onset neonatal Group B Streptococcus infection: management guideline for women in labour. (WDHB-7073). Whanganui, New Zealand: Whanganui District Health Board

Women's Health Unit. (2016). Guideline: 1. Management rupture of membranes 2. Antepartum/intrapartum Group B Streptococcus (GBS). (MDHB-7066). Palmerston North, New Zealand: MidCentral District Health Board 


\section{Appendix 3: Health and Disability Ethics Committee response.}

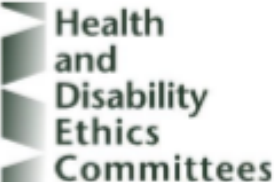

9 November 2018

Mrs Rebecca Hay

Victoria University of Wellington

Dear Mrs Hay,

Study title: Management of prelabour rupture of membranes at term at Northland District Health Board

Thank you for emailing HDEC a completed scope of review form on 01 November 2018. The Secretariat has assessed the information provided in your form and supporting documents against the Standard Operating Procedures.

Your study will not require submission to $\mathrm{HDEC}$ as, on the basis of the information you have submitted, it does not appear to be within the scope of HDEC review. This scope is described in section three of the Standard Operating Procedures for Health and Disability Ethics Committees.

Your study meets the student-led research exemption criteria described below. Your scope of review form described an observational research project for the attainment of a Master's degree.

For the avoidance of doubt a study conducted wholly or principally for the purposes of an educational qualification requires HDEC review only if it:

- is an intervention study, or

- is not conducted at or below a Master's level.

If you consider that our advice on your project being out of scope is in incorrect please contact us as soon as possible giving reasons for this.

This letter does not constitute ethical approval or endorsement for the activity described in your application, but may be used as evidence that HDEC review is not required for it.

Please note, your locality may have additional ethical review policies, please check with your locality. If your study involves a DHB, you must contact the DHB's research office before you begin. If your study involves a university or polytechnic, you must contact its institutional ethics committee before you begin.

Please don't hesitate to contact us for further information.

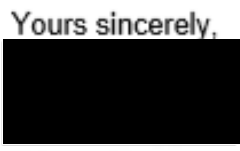

Tristan Katz

Assistant advisor

Health and Disability Ethics Committees hdecs@moh.govt.nz 


\section{Appendix 4: Victoria University of Wellington Human Ethics Committee response}

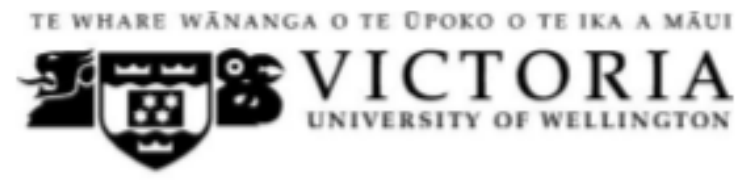

MEMORANDUM

\begin{tabular}{l|l}
\hline TO & Rebecca Barbara Hay \\
\hline FROM & Dr Judith Loveridge, Convenor, Human Ethics Committee \\
\hline DATE & $20 / 12 / 2018$ \\
\hline PAGES & 1 \\
\hline SUBJECT & $\begin{array}{l}\text { Ethics Approval } \\
\text { Number: 000026842 } \\
\text { Title: Management of prelabour rupture of membranes at term, at } \\
\text { Northland District Health Board }\end{array}$ \\
\hline
\end{tabular}

Thank you for your application for ethical approval, which has now been considered by the Human Ethics Committee.

Your application has been approved from the above date and this approval is valid for three years. If your data collection is not completed by this date you should apply to the Human Ethics Committee for an extension to this approval.

Best wishes with the research.

Kind regards,

Judith Loveridge

Convenor, Victoria University of Wellington Human Ethics Committee 


\title{
Appendix 5 Locality Approval
}

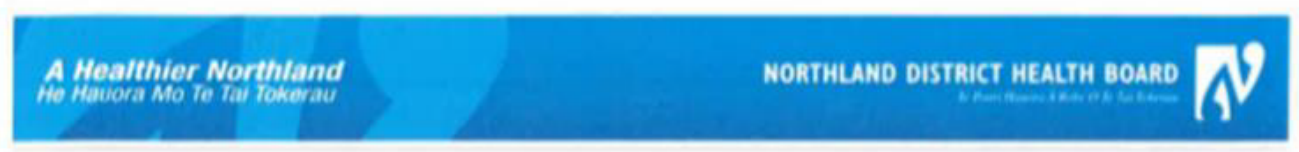

\section{Locality Assessment No. 2018-35}

\section{Locality Assessment Sign Off}

All research conducted in the Northland DHB must be conducted with the knowledge of the Northland DHB, and must meet all the requirements of the Heaith \& Disability Ethics Committees (HDECS), though not all research will require HDEC review.

\begin{abstract}
A locality assessment must be undertaken to review all research conducted at Northland District Health Board. Locality Assessments will consider resource implications, suitability of the local researcher and research environment, and cultural issues.
\end{abstract}

\section{Part One: General}

Full project titie:

Management of pre labour rupture of membranes at Northland District Health Board

Short project title:

Management of pre labour rupture of membranes at Northland District Health Board

Locality to be assessed:

\section{Northland}

Brief outline of study:

The research aims:
- To identify and review clinical processes against NDHB PROM policy standards via clinical audit

- Review outcomes for women and babies affected by PROM

- compare policies and outcomes with those of wider NZ

- to place my findings within the demographic context of Northland and NDHB

- consideration of what works well, and what could be improved upon

Principal investigator (for this locality):

Rebecca Hay

Contact details:

Email :

Work:

Personal

Other local investigators (list all at this site):

Deb Pittam, Maternity Services, Northland DHB

Contact details: 
Part Two: Locality Issues

Identify any local issues and specify how these issues will be addressed.

1. Sultability of local researcher

For example, are all roles for the investigator(s) at the local site appropriate<smiles></smiles>
(ie, has any conflict the investigator might have between her or his local roles in research and in patient care been adequately resolved)?

2. Suitability of the local research environment

a) Are all the resources (other than funding that is conditional on ethical

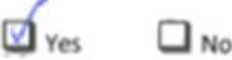
approval) and/or facilities that the study requires appropriate and available (for example, is staffing adequate? is this site accessible for mobilityimpaired people where necessary)?

b) Have all potentially affected managers of resources such as clinical records or $\nabla_{\text {Yes }} \square_{\text {No }}$ laboratory managers been notified?

3. Have issues such as cultural issues specific to this locality or to people being recruited at this locality been addressed?

4. Have the local investigator contact detalls and other important contact detalls been provided to the locality organisation for checking?

5. Has the local investigator been advised of the unacceptability of using personal hand-held devices to photograph identifiable patient information?

\section{Part Three: Declaration by locality organisation}

I am authorised to complete locality approval on behalf of this locality organisation. I understand that I may withdraw locality approval if any significant local concerns arise. I agree to advise the principal investigator and then the relevant ethics committee should this occur.

I confirm the organisation has sufficient indemnity insurance to compensate participants for harm that does not qualify for compensation under the Iniury Prevention. Rehabilitation and Compensation Act 2001.

Signature:

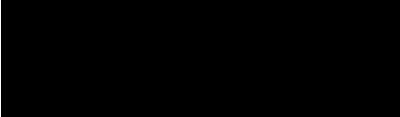

Date:

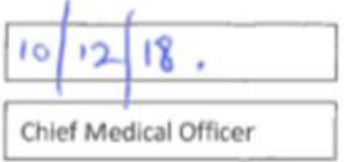

Name:

Northland District Health Board, Private Bag 9742, Whangarei 0148

Contact details: 


\section{Appendix 6: Data Collection Tool}

Study ID Number

Data collection tool: PROM at NDHB

\begin{tabular}{|c|c|c|c|}
\hline \multicolumn{4}{|l|}{ Demographics } \\
\hline \multicolumn{4}{|l|}{ Maternal age (years) } \\
\hline Maternal ethnicity & $\begin{array}{l}\text { Māori } \\
\text { European } \\
\text { Pacific Peoples }\end{array}$ & $\begin{array}{l}\text { Asian } \\
\text { Indiar } \\
\text { Other }\end{array}$ & Indian) \\
\hline \multicolumn{4}{|l|}{ Maternal BMI } \\
\hline \multicolumn{4}{|l|}{ Parity } \\
\hline \multicolumn{4}{|l|}{ Gestation } \\
\hline Smoker & \multicolumn{3}{|l|}{ YES / NO } \\
\hline $\begin{array}{l}\text { Evidence of LMC registration } \\
\text { prior to } 12 \text { weeks gestation }\end{array}$ & \multicolumn{3}{|l|}{ YES / NO } \\
\hline Booked LMC & Unbooked & Independent MW & Community MW \\
\hline $\begin{array}{l}\text { Did woman engage with care } \\
\text { post ROM, but before labour? }\end{array}$ & \multicolumn{3}{|l|}{ YES / NO } \\
\hline $\begin{array}{l}\text { Evidence of LMC visit post } \\
\text { ROM? }\end{array}$ & \multicolumn{3}{|l|}{ YES / NO } \\
\hline $\begin{array}{l}\text { Main maternity carer in } \\
\text { established labour }\end{array}$ & $\begin{array}{l}\text { LMC Midwife } \\
\text { Back up Midwife }\end{array}$ & $\begin{array}{l}\text { Core } \mathrm{N} \\
\text { Other: }\end{array}$ & Core Midwife \\
\hline $\begin{array}{l}\text { Care handed over during labour } \\
\text { (prior to birth) from Independent } \\
\text { to Core Midwife? }\end{array}$ & \multicolumn{3}{|l|}{ YES / NO } \\
\hline \multicolumn{4}{|l|}{ Intended place of birth } \\
\hline \multicolumn{4}{|l|}{$\begin{array}{l}\text { Distance from home to intended } \\
\text { place of birth }\end{array}$} \\
\hline \multicolumn{4}{|l|}{ Actual birth facility } \\
\hline $\begin{array}{l}\text { Distance from home to } \\
\text { Whangarei Base Hospital }\end{array}$ & $\begin{array}{l}0-49 \mathrm{~km} \\
50-99 \mathrm{~km} \\
100-149 \mathrm{~km}\end{array}$ & $\begin{array}{l}150-19 \\
200 \mathrm{~km}\end{array}$ & \\
\hline Fetus alive at ROM? & YES / NO & iscontinue data coll & \\
\hline
\end{tabular}




\begin{tabular}{|c|c|c|}
\hline \multicolumn{3}{|c|}{ Time factors and decision making } \\
\hline & DATE & TIME \\
\hline \multicolumn{3}{|l|}{ Membrane rupture } \\
\hline \multicolumn{3}{|l|}{ Labour established } \\
\hline \multicolumn{3}{|l|}{ Birth } \\
\hline \multicolumn{3}{|l|}{$\begin{array}{l}\text { Duration of membrane rupture } \\
\text { to established labour (HH:MM) }\end{array}$} \\
\hline \multicolumn{3}{|l|}{$\begin{array}{l}\text { Duration from membrane } \\
\text { rupture to birth (HH:MM) }\end{array}$} \\
\hline Group B Strep status at ROM & $\begin{array}{l}\text { low risk } \\
\text { high risk } \\
\text { unknown }\end{array}$ & \\
\hline $\begin{array}{l}\text { Consultation with SMO within } \\
24 \text { hours? }\end{array}$ & YES / NO & SMO name: \\
\hline $\begin{array}{l}\text { Evidence of clinical decision- } \\
\text { making process }\end{array}$ & \multicolumn{2}{|l|}{ YES / NO } \\
\hline $\begin{array}{l}\text { Evidence of maternal input into } \\
\text { decision making }\end{array}$ & \multicolumn{2}{|l|}{ YES / NO } \\
\hline Method of diagnosis of ROM & $\begin{array}{l}\text { Maternal Hx } \\
\text { Visible liquor }\end{array}$ & $\begin{array}{l}\text { Speculum } \\
\text { Other: }\end{array}$ \\
\hline $\begin{array}{l}\text { Appropriate for expectant } \\
\text { management? }\end{array}$ & \multicolumn{2}{|l|}{ YES / NO } \\
\hline Expectant management used? & \multicolumn{2}{|l|}{ YES / NO } \\
\hline \multicolumn{3}{|l|}{ Offered IOL as alternative? } \\
\hline \multicolumn{3}{|l|}{$\begin{array}{l}\text { Duration of expectant } \\
\text { management (HH:MM) }\end{array}$} \\
\hline \multicolumn{3}{|l|}{$\begin{array}{l}\text { Reasons stated if abandoning } \\
\text { expectant management }\end{array}$} \\
\hline Notes around decision making: & & \\
\hline
\end{tabular}


Study ID Number

\begin{tabular}{|c|c|c|c|}
\hline \multicolumn{4}{|c|}{ Maternal Labour and Birth Data } \\
\hline \multirow[t]{2}{*}{ Was labour induced? } & \multirow{2}{*}{$\begin{array}{l}\text { YES / NO } \\
\text { PGE2 Gel }\end{array}$} & \multicolumn{2}{|l|}{ Date and time IOL: } \\
\hline & & $\begin{array}{l}\text { ARM prior to } \\
\text { labour }\end{array}$ & Syntocinon IV \\
\hline \multirow[t]{2}{*}{ Was labour augmented? } & \multicolumn{3}{|l|}{ YES / NO } \\
\hline & \multicolumn{2}{|l|}{ ARM in labour } & Syntocinon IV \\
\hline \multirow{2}{*}{$\begin{array}{l}\text { Was IAP given to the mother } \\
\text { prior to birth? }\end{array}$} & YES / NO & \multicolumn{2}{|c|}{ Date/time IAP initiated: } \\
\hline & \multicolumn{2}{|c|}{ Duration ROM to IAP __ } & Number of doses of IAP: \\
\hline Date/time of first VE & & & \# VE in labour: \\
\hline \multicolumn{4}{|l|}{$\begin{array}{l}\text { Highest maternal } \\
\text { temperature in labour }\end{array}$} \\
\hline Chorioamnionitis & No & Suspected & Diagnosed \\
\hline $\mathrm{PPH}$ & No & $500-999 \mathrm{mls}$ & $1000+\mathrm{mls}$ \\
\hline Liquor: & \multicolumn{2}{|l|}{$\begin{array}{l}\text { Clear } \\
\text { Blood stained }\end{array}$} & $\begin{array}{l}\text { Thin/old meconium } \\
\text { Thick/fresh meconium }\end{array}$ \\
\hline Liquor quantity, if noted & Scant & Copious & Other: \\
\hline Pain relief used: & $\begin{array}{l}\text { Nil } \\
\text { Water } \\
\text { TENS }\end{array}$ & $\begin{array}{l}\text { Entonox } \\
\text { Massage/Rongoa } \\
\text { Pethidine }\end{array}$ & $\begin{array}{l}\text { Epidural } \\
\text { Spinal } \\
\text { Other: }\end{array}$ \\
\hline Mode of Delivery & $\begin{array}{l}\text { Vaginal } \\
\text { Ventouse } \\
\text { Forceps }\end{array}$ & $\begin{array}{l}\text { EM LSCS } \\
\text { EL LSCS } \\
\text { Waterbirth }\end{array}$ & Reason for CS: \\
\hline Episiotomy? & \multicolumn{3}{|l|}{ YES / NO } \\
\hline Perineal Trauma & $\begin{array}{l}\text { Intact } \\
\text { Labial Grazes } \\
\text { 1st degree }\end{array}$ & \multicolumn{2}{|l|}{$\begin{array}{l}\text { 2nd degree } \\
\text { 3rd degree } \\
\text { 4th degree }\end{array}$} \\
\hline Endometritis & No & Suspected & Diagnosed \\
\hline \multicolumn{4}{|l|}{$\begin{array}{l}\text { Maternal stay in facility } \\
\text { (days) }\end{array}$} \\
\hline Maternal mortality & YES / NO & Comment: & \\
\hline
\end{tabular}


Study ID Number

\begin{tabular}{|c|c|c|c|c|}
\hline \multicolumn{5}{|l|}{ Neonatal Outcomes } \\
\hline Birthweight (g) & & & \multicolumn{2}{|l|}{ Centile: } \\
\hline Neonatal APGAR Scores & $1 \mathrm{~min}$ & 5 mins & \multicolumn{2}{|l|}{$10 \mathrm{mins}$} \\
\hline Assigned Gender & Female & Male & \multicolumn{2}{|c|}{ Unassigned } \\
\hline Resuscitation required at birth? & \multicolumn{2}{|l|}{ YES / NO } & \multicolumn{2}{|l|}{ Details: } \\
\hline $\begin{array}{l}\text { Admission to SCBU for } \\
\text { respiratory support }>4 \text { hours? }\end{array}$ & \multicolumn{4}{|l|}{ YES / NO } \\
\hline \multicolumn{5}{|l|}{ Duration of SCBU admission } \\
\hline Referred to tertiary care & \multicolumn{4}{|l|}{ YES / NO } \\
\hline Respiratory diagnosis & TTN & RDS & \multicolumn{2}{|l|}{ Other } \\
\hline Treatment with IV antibiotics? & \multicolumn{4}{|l|}{ YES / NO } \\
\hline \multirow[t]{3}{*}{ Diagnosis with EOGBS? } & \multicolumn{4}{|l|}{ YES / NO } \\
\hline & \multicolumn{2}{|l|}{ Probable } & \multicolumn{2}{|l|}{ Definite } \\
\hline & \multicolumn{2}{|l|}{ Meningitis } & \multicolumn{2}{|c|}{ Pneumonia } \\
\hline Diagnosis of late onset GBS & \multicolumn{4}{|l|}{ YES / NO } \\
\hline Neonatal Mortality & NO & Stillbirth & & Neonatal demise \\
\hline \multicolumn{5}{|l|}{ Comment on mortality event } \\
\hline \multicolumn{5}{|l|}{$\begin{array}{l}\text { Any other comments about this } \\
\text { case (maternal or neonatal) }\end{array}$} \\
\hline \multicolumn{5}{|l|}{ Neonatal stay in facility (days) } \\
\hline \multirow{2}{*}{$\begin{array}{l}\text { Breastfeeding on discharge } \\
\text { from facility }\end{array}$} & \multicolumn{2}{|l|}{ Exclusive } & \multicolumn{2}{|l|}{ Partial } \\
\hline & Fully & & Artificial & \\
\hline
\end{tabular}




\section{Appendix 7: NDHB error log}

\author{
Study ID NHI \\ number number \\ Description of error
}

3

7

16

20

21

24

25

27

30

32

33

35

42

44

45

48 for breech

Not PROM, error in MP data

excluded as PPROM $36+5$ gestation

Baby resuscitation not recorded in Mat+.

Not PROM, error in MP data; 3hrs from ROM to birth

section

Time of birth noted on LR incorrectly and repeated on MP

Baby resuscitation not recorded in Mat+. prior to EMLSCS birth; Mat+ time of ROM incorrect.

excluded as PPROM $35+5$ gestation

Morphine given as pain relief, not recorded on LR or Mat+ not noted on LR and not included in postnatal care plan.

$01: 14$, same as time of birth as baby was born in the caul. birth 0654 but recorded in Mat+ as 0554

ROM time recorded as 00:00 but was actually 22:15

Morphine given as pain relief, not recorded on LR or Mat+ arrived with $\mathrm{Hx}$ of ROM and no ARM performed at any time.

Mat+ records ROM 25/8/18 but was 26/8/18

Morphine given as pain relief, not recorded on LR or Mat+

Data entry error: false date and time created for ROM during elective section

No PROM, ARM at 0900 on 14/12/19 but LR and Mat + give date as 13/12/18.

Error in LR

Error in

Morphine

Excluded

entries Mat+ data documentation from data

issues

Clinical notes record ROM 23/4/19 2400; LR 23/4/19 2410; Mat+ 23/4/19

0410. Clinical notes appear correct date and time but not well documented

Date/time error written on LR and Mat+, both 0300 but ROM 1500.

Data entry error: false date and time created for ROM during semi acute

Data entry error: date and time created for full dilation and pushing 24 hours

Time of ROM written on LR as 0800 instead of 0500; carried over to MP

LR states ARM at 4cm; however no reference to this in clinical notes

LR and Mat+ record a date and time given by woman on presentation for query SROM, however ROM not investigated/proven effectively. ARM done in labour with meconium liquor; date and time of that are correct on Mat +. Mec liquor

ROM date and time on Mat+ states 16/7/19 00:00; but correct time is 17/7/19

Time of ROM written on LR as 2000; but 2200 in the clinical notes. Time of

Mat+ states labour est and fully dilated 22/6/18 00:00 - never happened.

Mat + states hospital admission was $16 / 5 / 190800$, but was $17 / 5 / 190800$.

However inpatient front sheet dated correctly. Also; LR states there was an ARM but no ARM recorded in clinical notes. Was an IOL for postdates, but

ROM time AND Labour established times not recorded in Clinical notes or LR, but created in Mat+. ROM near time of birth (not documented well).

Clinical notes do not record time of ROM, but was close to time of birth. Is not stated on LR but a date and time are given on Mat + of 29/10/18 00:00, giving false impression of PROM 

record of either in clinical notes. No PROM. incorrectly recorded on LR and Mat+ as 22:00, but not present in WBH at that time and not documented in clinical notes as that time. Could be the next day at either 01:00 or 02:00

Morphine not recorded on Mat+ or handed over; 2 doses shortly prior to birth.

Time of SROM consistently 1430 in clinical notes, but 0200 on LR \& Mat +

Fully dilated $24 / 9 / 1800.00$ but never occurred; birthed at 21.36 . Also augmentation stated on LR and Mat + but was IOL as syntocinon started prior to labour establishing - however unclear when labour established; 0900 entry not related to standard description of labour; 1220 would be better.

excluded as PPROM $36+6$ gestation

Fully dilated time stated but didn't occur. Also recorded as acute CS but was in established labour therefore emergency.

Morphine given, not recorded on LR or Mat +

LR states gestation $37+1$ but was $40+5$. Appears to have been written during a previous presentation for query SROM which was disproved.

States labour est when woman not contracting regularly and $\mathrm{Cx} 1 \mathrm{~cm}$ dilated.

Morphine given but not recorded on LR or Mat +

excluded as PPROM 35+6 gestation

morphine given but not recorded on LR or Mat +

Baby infant record and clinical notes states PEEP from 4 to 10 mins of age in air. Mat+ only states oxygen used, no further details.

Morphine given $\mathrm{x} 2$, not noted in LR or Mat +

Morphine given, not noted on LR

Time of ROM not recorded in clinical notes or on LR, entered as 00:00 in Mat+, unclear if correct.

Labour est time not documented in clinical notes and clearly incorrect on LR. May be correct in Mat +, but unknown.

ROM time blank on LR and 00:00 on Mat+. Woman reported SRM 12/4 0530, clear liquor. ARM in clinical notes 12/4 1556, not recorded on LR or Mat+. Care questionable? Eleven digital VEs, no IAP.

Morphine given $x 4$, not recorded on LR. IAP given, not recorded on LR. IOL not recorded on LR (but is in Mat+)

Clinical notes don't record ROM except at 1/2/19 0135 "....arrived at BOI in established labour, wanting to push. Had SROM copious amount blood stained in car on the way to hospital." LR states ROM 31/1/19 0455, as does Mat+, but no clinical notes reflect this. Conclude not PROM.

APGAR of 9 at 1 min but resps not est until 2 mins with suction, oxygen, ambubag and CPAP given? Synto infusion use not recorded in LR or Mat+ Mat+ records SROM on 7/10/18 0132, but clinical notes and LR stage 8/10/19 0132, 2 mins prior to birth

Clinical notes entries $0130-0400$ unsigned by unidentified core mw giving LMC a break.

Clinical notes and LR state ROM 27/8/18 0845 but Mat+ states 26/8/19 00:00. 
Appendix 7

Mat + states labour established 7/8/18 0730 but LR states $8 / 8 / 180730$. There

is no documentation in clinical notes at this time but an entry at $8 / 8 / 180010$ states contractions 2-3 min lasting a minute and VE of $3 \mathrm{~cm}$ fully effaced and station 0. Next exam at $0900=\mathrm{Cx} 7 \mathrm{~cm}$ dilated. Also Mat+ documents a waterbirth but was a land birth with water used for pain relief in 1st stage only.

ROM 4/9/18 2200 recorded in clinical notes but 5/9/19 2200 in Mat+. No date/time recorded on LR

ROM 15/8/18 11.00 recorded in clinical notes and LR; but Mat+ records 17/8/18 11.00. Other date errors on LR.

Clinical notes for 26/10/18 1116 state @?SROM leaking fluid from 24/10/18 0200 hours. LR and Mat+ state 26/10/18 0200 and this error is then continued through clinical notes. IOL offered based on the later date but actually 5 days after $26 / 10 / 18$.

ROM 12/5/19 1800 in clinical notes but speculum exam done 14/5/19 1740 with meconium liquor found; ARM performed 15/5/19 1545. LR and Mat+ record ROM at time of ARM

Clinical notes record ROM 10/4/19 1000 but LR and Mat+ both state 14/4/19, with varying times of 0428 and 0450 . Unclear which is correct; 0428 correlates to birth time of Twin 1 and 0450 could relate to ARM for Twin 2.

Clinical notes record ROM 19/4/19 2330 with fetal hair visible on speculum; LR and Mat+ record ROM 20/4/19 1230 - nothing in clinical notes at this time.

SROM queried 16/6/8 1900 and this date used in LR - not found on exam at that time, but confirmed (with no further events of note in maternal history) 18/6/19 1345; not recorded Mat+ 